\title{
Imaginary-Time Approach to the Kondo Effect out of Equilibrium
}

\author{
Dissertation \\ zur Erlangung des mathematisch-naturwissenschaftlichen \\ Doktorgrades \\ "doctor rerum naturalium" \\ der Georg-August-Universität Göttingen
}

\section{VOn \\ Andreas Dirks}

aus Duderstadt

Göttingen, 2012 
Referent:

Prof. Dr. Thomas Pruschke

Korreferent:

Prof. Dr. Fakher F. Assaad

Tag der mündlichen Prüfung: 19.06.2012 
„Die Mathematik ist Tapferkeitsluxus der reinen Ratio, einer der wenigen, die es heute noch gibt. Auch manche Philologen treiben Dinge, deren Nutzen sie wohl selbst nicht einsehen, und Briefmarken- oder Krawattensammler noch mehr. Aber das sind harmlose Launen, die sich fern von den ernsten Angelegenheiten unseres Lebens abspielen, während die Mathematik gerade dort einige der amüsantesten und schärfsten Abenteuer der menschlichen Existenz umschließt."

Robert Musil: Der mathematische Mensch (1913) 


\section{Contents}

$\begin{array}{ll}\text { 1. Introduction } & 1\end{array}$

2. Kondo Effect in Mesoscopic Systems 3

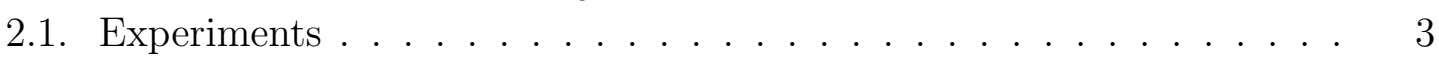

2.1.1. Setup of a single-electron transistor . . . . . . . . . . . 3

2.1.2. Experimental Observables . . . . . . . . . . . . . . 4

2.2. Anderson Impurity Model . . . . . . . . . . . . . . . . . . . . . . . 5

2.2.1. Kondo Effect . . . . . . . . . . . . . . . . . 6 6

2.2.2. Linear Response Theory . . . . . . . . . . . . . . . . . . 9

2.3. Nonequilibrium Perturbation Theory . . . . . . . . . . . . . . . . 10

2.3.1. Fundamental correlation functions . . . . . . . . . . . . 10

2.3.2. Application to Nonequilibrium Kondo Systems . . . . . . . . . 12

2.4. Summary . . . . . . . . . . . . . . . . . . . . 12

3. The Matsubara-Voltage Formalism 13

3.1. Quantum Theory of Scattering . . . . . . . . . . . . . . . . . 13

3.1.1. Potential scattering of a single particle . . . . . . . . . . . . . . 13

3.1.2. Many-body scattering theory . . . . . . . . . . . . 18

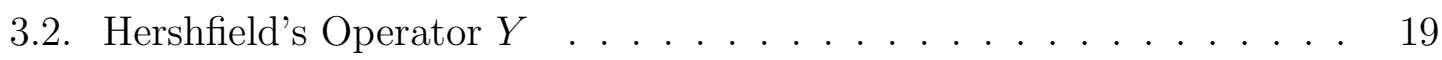

3.2.1. Outline of the formalism . . . . . . . . . . . 20

3.2.2. Application of the approach . . . . . . . . . . . . . 21

3.3. Matsubara-Voltage Approach . . . . . . . . . . . . . . . . . 22

3.3.1. Structure of Expectation Values . . . . . . . . . . . . . . . 23

3.3.2. Matsubara Voltage Substitution . . . . . . . . . . . . . . . 24

3.3.3. Effective Hamiltonian . . . . . . . . . . . . . . . . . . . . 25

3.4. Physical Observables ... . . . . . . . . . . . . . . 26

3.4.1. Range of applicability . . . . . . . . . . . . . . . . 26

3.4.2. Structure of the perturbation expansion . . . . . . . . . . . . 28

3.4.3. Atomic Limit . . . . . . . . . . . . . . . . . . 36

3.4.4. Symmetry Relations . . . . . . . . . . . . . . . . 39

3.4.5. Analytic Structure of Static Observables . . . . . . . . . . . . 39

3.5. Summary . . . . . . . . . . . . . . . . . . . . 40 
4. Quantum Monte Carlo 43

4.1. Algorithms . . . . . . . . . . . . . . . . . . . . . . 43

4.1.1. Interaction Expansion . . . . . . . . . . . . . . . 43

4.1.2. Hybridization Expansion . . . . . . . . . . . . . . . . . 45

4.2. Application to Matsubara-Voltage Formalism . . . . . . . . . . . . . 46

4.2.1. CT-AUX . . . . . . . . . . . . . 46

4.2 .2$. CT-HYB . . . . . . . . . . . . . . . . . 48

4.3. Computational Performance . . . . . . . . . . . . . . . . . . . . . . 49

4.4. Summary . . . . . . . . . . . . . . . . . . . 50 50

5. Dynamic Expectation Values and Several Complex Variables 53

5.1. Domains of Holomorphy and Biholomorphic Transformations . . . . . 54

5.1.1. One-dimensional function theory . . . . . . . . . . . 54

5.1.2. Multi-dimensional function theory . . . . . . . . . . . 56

5.2. Boundary value problems . . . . . . . . . . . . . . . . . 57

5.2.1. The Shilov Boundary . . . . . . . . . . . . . . 58

5.3. Holomorphic Structure of the Green's Functions . . . . . . . . . . . . 60

5.3.1. Resulting Mathematical Assumptions . . . . . . . . . . . . 61

5.3.2. Justification of assumption 3' . . . . . . . . . . . . . . . . . . . . . . 62

5.4. Tubular Cone Domains ("Wedges") . . . . . . . . . . . . . . . 63

5.4.1. Geometry of the Cones . . . . . . . . . . . . . . 63

5.4.2. Analytic Structure and Biholomorphic Equivalence Classes . . 64

5.4.3. Bergman-Weil Representations . . . . . . . . . . . . . . 65

5.4.4. Cauchy-Bochner Integral Representation . . . . . . . . . . . . 67

5.4.5. The tubular octant and Equivalence to the Bicylinder . . . . . 68

5.5. Vladimirov's Integral Formula . . . . . . . . . . . . . . . . . . . 70

5.5.1. Herglotz-Nevanlinna Representation $(d=1)$. . . . . . . 70

5.5.2. Functions with positive real or imaginary part in $T^{C}$. . . . 72

5.5.3. Vladimirov's Kernel Functions for $T^{C} \ldots$. . . . . . . . . . 73

5.5.4. Vladimirov's Theorem . . . . . . . . . . . . . . . 73

5.6. Application to the Green's function . . . . . . . . . . . . . . . . . 74

5.6.1. Kernels for $\vartheta=0 \ldots \ldots 75$

5.6.2. Operator Notation . . . . . . . . . . . . . . . . . . . 75

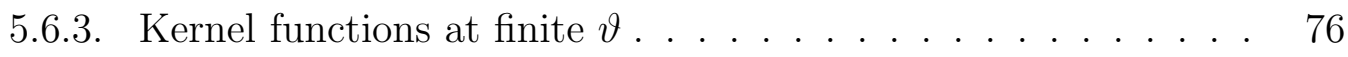

5.6.4. Edge Properties of $G_{0} \ldots \ldots \ldots 77$

5.7. Summary . . . . . . . . . . . . . . . . . . . . 78

6. Bayesian Inference for Numerical Analytic Continuation $\mathbf{7 9}$

6.1. Static Observables . . . . . . . . . . . . . . 82

6.1.1. Double Occupancy ................... 84

6.1.2. Magnetic Susceptibility . . . . . . . . . . . . . . . . . . . 85

6.2. Dynamic Observables . . . . . . . . . . . . . . . . . . . 87 
6.2.1. Single-Wedge Continuation . . . . . . . . . . . . . . . . 87

6.2.2. Geometry of the Single-Wedge Inference Problem . . . . . . . 98

6.3. Summary . . . . . . . . . . . . . . . . 101

7. Generalized Approach to the Double Analytic Continuation 103

7.1. Bogolyubov's edge-of-the-wedge theorem . . . . . . . . . . . . . . 103

7.2. Systematic extension of the continuation procedure . . . . . . . . . . 104

7.2.1. Shared real part on edges . . . . . . . . . . . . 105

7.2.2. A-priori constraints to the shared-real-part assumption . . . . 105

7.2.3. Functional-analytic consequences . . . . . . . . . . . 106

7.2.4. Numerical implementation of $\mathcal{Q}_{r, \vartheta} \ldots \ldots$. . . . . . . . . 109

7.3. Results of the $\mathcal{Q}$-approach . . . . . . . . . . . . . . 112

7.3.1. Weak-Coupling Regime . . . . . . . . . . . . 113

7.3.2. Intermediate-Coupling Regime . . . . . . . . . . . . . . 115

7.3.3. Transport properties . . . . . . . . . . . . . . 116

7.4. Perspective: Exact $\mathcal{Q}$-approach . . . . . . . . . . . . 116

7.5. Summary . . . . . . . . . . . . . . . . . . . 120

$\begin{array}{ll}\text { 8. Conclusion } & 123\end{array}$

A. Uniqueness of Continuation $\quad 125$

A.1. Reconstruction of edge values using upper half planes . . . . . . . . . 126

A.2. Extending the unique range to the entire edge . . . . . . . . . 128

B. A-priori information about residual discontinuities 131

C. Commutator relations of $\mathcal{Q}_{\vartheta}^{\text {(edge) }} \quad \mathbf{1 3 5}$

C.1. Translational invariance . . . . . . . . . . . . . . . . . . 135

C.2. Scale invariance . . . . . . . . . . . . . . . . 137

C.3. Absence of rotational invariance . . . . . . . . . . . . . 137

D. MaxEnt implementation for data from multiple wedges 139

D.1. Application to the interacting model . . . . . . . . . . . . . . . 145

E. Numerical representation of the multi-wedge map $\mathcal{Q} \quad 149$

E.1. Analytic computation of three first integrals . . . . . . . . . . . . . . 149

E.1.1. Rational coefficients of the transformed edge test function . . 151

E.1.2. Composition of the rational coefficients . . . . . . . . . . 152

E.1.3. Contraction with the Poisson kernel . . . . . . . . . . . . 153

E.2. Numerical quadrature of the fourth integral . . . . . . . . . . . . 154

$\begin{array}{ll}\text { Bibliography } & 157\end{array}$ 


\section{Introduction}

The Kondo effect is a central phenomenon in the study of quantum-mechanical manybody correlations in electronic systems. It was observed in the study of dilute magnetic impurities in metals first $[1 ; 2]$. In these systems, surrounding electrons tend to screen the magnetic moment of the impurity by forming a spin-singlet state with impurity electrons. At low temperatures, this gives rise to unconventional changes in physical observables such as electrical resistivity as compared to normal metals.

Within a theoretical model it was pointed out by Jun Kondo that the effect is due to a non-perturbative many-body resonance [3]. It originates from resonant spinflip scattering of electrons. The adequate theoretical characterization of the manybody resonance is the so-called Kondo problem. It was solved by Wilson's numerical renormalization group which provides a controlled non-perturbative computation of physical observables $[4 ; 5]$. At present, numerous analytical and numerical methods are available and under further development for the understanding of the nuances of the Kondo effect [6].

More recently, the Kondo effect was also observed in mesoscopic devices [7;8]. It is possible to study it far beyond thermal equilibrium by applying a bias voltage to the device. Its relevance in mesoscopic systems is particularly high due to conceptual connections to quantum computers and spintronics $[9 ; 10]$.

For the new setups in mesoscopic systems, a further conceptual difficulty was added to the Kondo problem. The state imposed by the nonzero source-drain voltage must be described theoretically by a non-equilibrium quantum-statistical ensemble, the mere definition of which is a challenge for theoreticians. Standard approaches from the equilibrium setup are no longer applicable. Several methods for the nonequilibrium Kondo problem have been developed. These include perturbative approaches [11-13], renormalization groups [14-18], and numerical techniques [19-26]. It has been a controversial debate to which extent the many-body coherence of the Kondo effect is maintained under non-equilibrium conditions. Presently available approaches may be subdivided into two classes. First, it is possible to approach the stationary state imposed by the bias voltage by switching a parameter in the Hamiltonian and computing the time-evolution to a steady state. Here, the physical relaxation dynamics are of additional interest. Second, the stationary state may be described directly by techniques involving scattering-states bases.

In the present thesis, an approach proposed by Han and Heary is investigated [27]. It uses a basis of noninteracting scattering states as a starting point in order to map the non-equilibrium Kondo system to an infinite set of equilibrium systems. 
Analytic continuation with respect to an imaginary voltage which parametrizes the equilibrium systems retrieves the non-equilibrium case. The major advantage of the approach is that it facilitates the employment of numerous well-understood and numerically controlled equilibrium techniques such as quantum Monte-Carlo for a study of the non-equilibrium problem.

The first chapter of the thesis briefly discusses experimental realizations of the Kondo effect in mesoscopic systems. As a standard model description, the singleimpurity Anderson model is introduced, and the basic theoretical starting point is reviewed. Starting from this, the Matsubara-voltage formalism is presented in the second chapter. Its inheritances from previous formal approaches by Zubarev and Hershfield are discussed [28; 29]. Recent insights on the validity of the approach are discussed. In chapter 4, the effective-equilibrium systems are solved numerically with recently developed continuous-time quantum Monte-Carlo methods in order to produce highly accurate data [30-33]. The latter are subject to further analysis in the subsequent chapters. Chapter 5 introduces mathematical concepts from the function theory of several complex variables and applies them to the structure of dynamical expectation values and transport properties in imaginary-voltage theory. An integral representation by Vladimirov is found to be of fundamental importance [34]. In chapter 6 , the numerical analytic continuation of observables from imaginaryvoltage theory will be discussed. The standard approach to analytic continuation of quantum Monte-Carlo data, based on Bayesian inference [35], is first applied to static observables. Vladimirov's integral kernel is then used to derive first results for dynamic observables and conductivity properties. This is followed by a discussion of possible generalizations of the continuation algorithm for dynamic observables and conductivity properties in chapter 7 . Based on a continuity assumption for the analytic structure of the correlation functions, a more effective integral kernel is derived and invoked for the computation of non-equilibrium correlation functions and transport properties. Chapter 8 concludes the thesis. 


\section{Kondo Effect in Mesoscopic Systems}

In this chapter, experimental realizations of nonequilibrium Kondo systems in quantum dots are discussed. An introduction to the theoretical description of the Kondo effect in such systems via the Anderson impurity model is given. The Kondo effect in equilibrium is outlined by means of its historical development, its phenomenology, and its theoretical treatment. Particular emphasize is put on perturbative approaches, because the imaginary-voltage theory employed in the present thesis relies on perturbative arguments. The Keldysh real-time approach to the definition of a nonequilibrium system starting from a well-defined system is briefly introduced. This connects to the next chapter in which the real-time evolution is bypassed via the introduction of imaginary voltages.

\subsection{Experiments}

Our discussion of experimental realizations of strongly correlated electrons in mesoscopic systems will be limited to the systems investigated by Cronenwett et al. and Goldhaber-Gordon et al., who were first able to observe an out-of-equilibrium Kondo effect in quantum dots [7; 8]. A typical device, based on a GaAs/AlGaAs heterostructure, is displayed in figure 2.1. ${ }^{1}$ Apart from the semiconductor heterostructures, the effect was also observed in carbon nanotubes [36; 37].

\subsubsection{Setup of a single-electron transistor}

The single-electron transistor in figure 2.1 is comprised of a GaAs and an AlGaAs layer, between which a two-dimensional electron gas (2DEG) is formed. Electrodes on the surface of the sample are negatively charged in such a way that the 2DEG is depleted in certain geometrically well-defined regions. This depletion leads to a confinement of 2DEG-electrons to orbitals with sharp well-separated energy levels $\varepsilon_{d}$ in the central region of the device, the quantum dot. Tunneling through the depleted region to the unconfined parts of the 2DEG leads to a finite life-time, i.e. a finite energy-level broadening $\Gamma$, of the dot electrons. The unconfined parts of the 2DEG

\footnotetext{
${ }^{1} \mathrm{~A}$ detailed review about the experimental realization of such devices may be found in the article by Hanson et al. [10].
} 


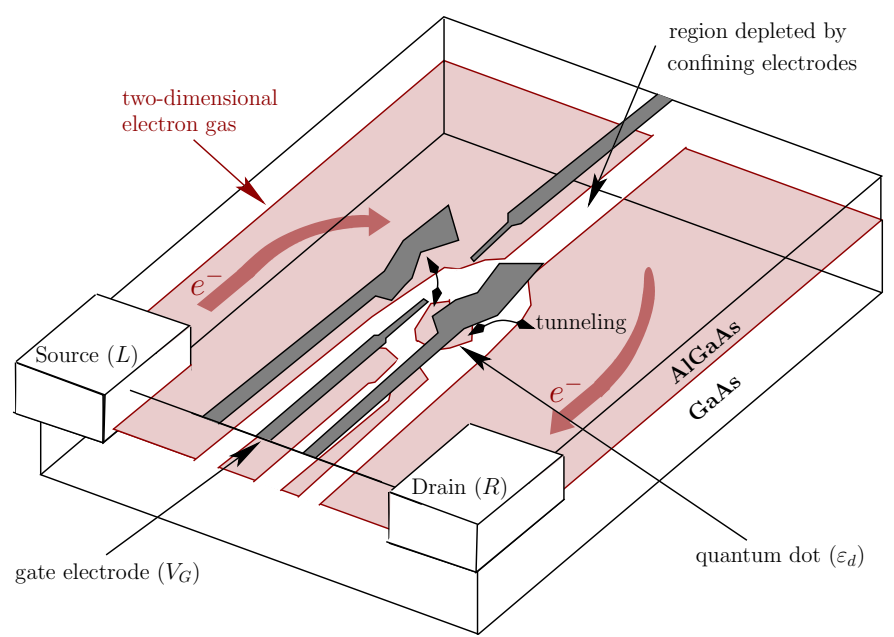

(a) Schematic drawing of setup

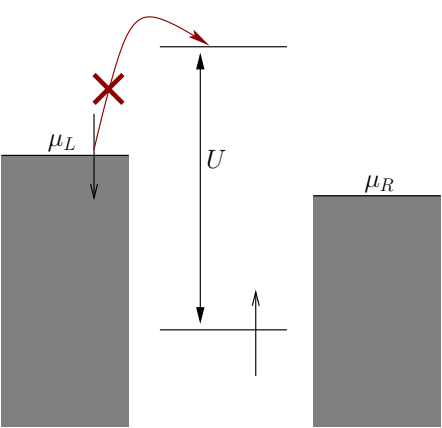

(b) Coulomb blockade

Figure 2.1: Coulomb blockade physics in a single-electron transistor [7; 10].

being attached to source and drain electrodes form source and drain reservoirs. The energy level(s) $\varepsilon_{d}$ of the dot device may be tuned with respect to the Fermi levels of source and drain reservoirs by the gate voltage $V_{G}$.

A further important energy scale is introduced by the strong confinement of electrons to the quantum dot. It is the Coulomb repulsion which leads to a finite capacitance when an electron is added to the dot due to a tunneling process. Although the capacitance consists of several contributions including capacitance with respect to the conduction leads, it effectively yields a repulsion energy $U$ of spin-up and down electrons within a spin-degenerate orbital [10]. The interplay of $U, \varepsilon_{d}$ and $\Gamma$ gives rise to another energy scale, the Kondo temperature $T_{K}$. It is associated to the emergence of a coherent many-body state leading to a strong quantum-mechanical entanglement of dot and lead electrons. The electrons form singlets which effectively screen the free spin on the orbital.

\subsubsection{Experimental Observables}

The observables which are measurable with state-of-the-art techniques are the current $J$ and the differential conductance $G=\frac{\mathrm{d} J}{\mathrm{~d} \Phi}$ as a function of the applied source-drain voltage $\Phi$. The magnetic susceptibility of the quantum dot as a function of a magnetic field is in principle an excellent probe for the presence of the Kondo state; cf. section 6.1. It is however not within the range of current experimental resolution. Similarly, it is presently impossible to determine the dot-electron spectral function rather directly via photoabsorption.

Nevertheless, the interplay of temperature, Kondo scale, bias voltage and the other energy scales leads to a striking behaviour of the conductance. When the Kondo scale 
is suppressed, transport behaviour is dominated by simple level shifts using the gate voltage. In this so-called Coulomb-blockade regime, transport of an electron from the source lead through the dot to the drain lead is possible whenever a quantum dot energy level crosses the window defined by the two Fermi energies of the leads. However, due to the Coulomb interaction $U$ of two electrons in an otherwise degenerate orbital, the energy level is effectively shifted whenever the considered orbital is singly occupied, cf. Fig. 2.1b. In the situation shown in the picture it is neither possible to remove or add an electron. The conductivity of the dot is zero, due to the Coulomb interaction.

When the system is cooled down below the Kondo temperature $T_{K}$ and if $e \Phi$ is not significantly larger than $k_{B} T_{K}$, a nonzero conductance arises in an otherwise unchanged system. This is the zero-bias anomaly due to the Kondo effect. Detailed studies of the Kondo effect as a function of bias voltage, magnetic field, gate voltage, temperature, and extensions of the quantum dot geometry are possible due to the well-tunable parameters [38]. An utterly new aspect is driving the Kondo effect far beyond thermal equilibrium by imposing the voltage bias.

\subsection{Anderson Impurity Model}

From the theoretical point of view, one finds that the study of transport through small mesoscopic systems such as quantum dots may be often reduced to the physics of quantum impurity models which involve a coupling to infinite source and drain leads. In many cases these leads can be simply described by Landau's Fermi liquid theory. Superconducting or strictly one-dimensional [39; 40] leads go beyond this picture and increase the physical complexity.

The standard model for transport through a small quantum dot with Kondo correlations is the single-level Anderson impurity model with two attached conduction leads [41]. Its Hamiltonian consists of terms for the local part $H_{\text {loc }}$ and the conduction, the term $H_{\text {leads }}$ for the conduction electrons, a hybridization term $H_{\text {hyb }}$, and a many-body interaction term $H_{\text {int }}$ :

$$
H=H_{\text {leads }}+H_{\text {loc }}+H_{\text {hyb }}+H_{\text {int }} .
$$

The conduction electrons carry a lead index $\alpha$, and an energy dispersion $\varepsilon_{\alpha k \sigma}$. The spin degree of freedom is the only local quantum number, i.e.

$$
H_{\mathrm{loc}}=\sum_{\sigma} \varepsilon_{d, \sigma} d_{\sigma}^{\dagger} d_{\sigma}
$$

Without a spin-orbit interaction, the hybridization to the conduction leads conserves 
the spin

$$
H_{\mathrm{hyb}}=\sum_{\alpha k \sigma}\left(V_{\alpha k \sigma} d_{\sigma}^{\dagger} c_{\alpha k \sigma}+\text { h.c. }\right)
$$

We will furthermore assume that the hybridization is energy-independent, i.e.

$$
V_{\alpha k \sigma}=t_{\alpha}
$$

Without an external magnetic field, the $d$-orbitals are spin-degenerate. The interaction term simply introduces a capacitive charging contribution

$$
H_{\mathrm{int}}=U \cdot\left(n_{d, \uparrow}-1 / 2\right)\left(n_{d, \downarrow}-1 / 2\right) .
$$

The case $U=0$ is usually referred to as the resonant-level model. The quadratic Hamiltonian allows a straightforward exact solution. In the limit of flat conduction bands associated to the source and drain leads, using the energy-independence of the hopping, one obtains an energy-independent broadening $\Gamma$ of the dot level spectral function. This is associated to the finite life-time of electrons due to the hopping.

\subsubsection{Kondo Effect}

In 1961, the Anderson impurity model was introduced by P. W. Anderson in order to study the specific heat and electrical resistivity anomalies which had been measured for diluted magnetic atoms in metals [42]. Despite the simple form, its physical content is highly nontrivial. At zero temperature the perturbation series with respect to $V / U$ includes logarithmic infrared divergencies. This is equivalent to the Kondo problem in the s-d exchange model [3]. The Anderson model exactly maps to the latter in the limit $U / V \rightarrow \infty$, through a Schrieffer-Wolff transformation [43].

It is often referred to as Kondo model and has the form

$$
H=\sum_{k \sigma} \varepsilon_{k \sigma} c_{k \sigma}^{\dagger} c_{k \sigma}+J \cdot \vec{S} \cdot \vec{s}
$$

where $\vec{s}$ denotes the net spin of the conduction electrons. $J$ represents a local coupling of the impurity spin $\vec{S}$ to the tight-binding chain of lead sites with local spin $\vec{s}$. The Anderson model extends the s-d model by allowing for charge fluctuations on top of the spin fluctuations.

The main reason why advance in the field has been both challenging but also physically enlightening may be found in the so-called Anderson orthogonality catastrophy. Anderson pointed out that the Fermi liquid ground state of the interacting Anderson model becomes orthogonal to the non-interacting one for sufficiently large interaction strengths and that the overlap decreases exponentially as a function of the interaction [44; 45]. One important consequence of the orthogonality catastrophy is the formation of a sharp Abrikosov-Suhl resonance next to the Fermi level [46; 47]. Its width, 
the Kondo temperature $T_{K}$, is a newly emerging energy scale. It is essentially ${ }^{2}$ given by the expression

$$
T_{K} \approx \frac{\sqrt{\Gamma U}}{2} \exp \left(\frac{\pi\left(\varepsilon_{d}-U / 2\right)\left(\varepsilon_{d}+U / 2\right)}{\Gamma U}\right)
$$

from Haldane's scaling theory [48]. The essential singularity for $\Gamma \rightarrow 0$ indicates that a perturbative expansion in $V / U$, or, equivalently for the Kondo model (2.6), in $J$ must be regularized. The non-crossing approximation (NCA) and its systematic extensions perform an infinite resummation of diagrams for this purpose [49-51].

\section{Phenomenology}

The Kondo effect can be described phenomenologically as follows [52]. At very high temperatures $T \gg T_{K}, k_{B} T \gg U$, one-particle dynamics dominates, due to the decoherence introduced by the strong thermal fluctuations. When the temperature is decreased, the departure of a spin-down electron from the impurity and the arrival of a spin-up electron in the impurity become correlated if a finite Coulomb interaction is present. The latter makes the simultaneous presence of up and down spins on the impurity energetically unfavorable and increasingly prefers sequential tunneling processes. This sequential appearance of spin-up and -down electrons effectively gives rise to a mutual spin flip of impurity and band electrons. With regard to in- and outcoming band electrons it gives thus rise to so-called spin-flip scattering. At lower temperatures $T \approx T_{K}$, the scattering becomes resonant, i.e. a many-body state forms in which scattered electrons scatter again coherently with the impurity. By this, the local spin is effectively screened by a cloud of conduction electrons. These resonant spin fluctuations are frozen out to the Kondo ground state as $T$ approaches zero. The many-body resonance gives rise to a sharp peak of width $T_{K}$ at the Fermi level in the spectral function of the impurity electron.

In the case in which the system is driven out of equilibrium due to a finite bias voltage, it is a matter of debate, to which extent the decoherence introduced by the bias voltage is analogous to that corresponding to the temperature of an equilibrium system. An alternative to such an effective-temperature phenomenology would be the partial maintenance of the Kondo coherence or other qualitatively new features. For example, some computations indicate a pinning of the Kondo resonance to the Fermi levels of each of the leads $[13 ; 17]$.

\footnotetext{
2 The exact prefactor of the exponential has been subject to dispute since it also depends on the precise definition of the width of a peak. Like many other low-energy properties it may be extracted accurately as a full width at half maximum from NRG computations [4].
} 


\section{Perturbative Approach in $U / V$}

As a next step, $U / V$ perturbative expansions will be discussed, whose applicability is crucial for a justification of the imaginary-voltage approach utilized in the present work $[27 ; 53 ; 54]$. A consequence of Anderson orthogonality is that not only perturbative expansions in $V / U$ become nontrivial (see above), but also with respect to $U / V$. Convergence is a priori not guaranteed. It was pointed out that in cases with linear dispersion relation the Anderson Model may be mapped to a one-dimensional system that can be solved exactly with a Bethe ansatz [55-59]. In this case the systematic expansions of zero-temperature spin and charge susceptibilities around the Hartree-Fock Hamiltonian with respect to $U / V$ by Yosida and Yamada prove to converge absolutely for arbitrary finite values of $U$ [60-62]. Further comparisons to results from Bethe ansatz showed that the same is true for the ground state energy [63]. Initial indications [64] for an only finite convergence radius $U_{\max }=2 \Gamma$ appear to be devitalized by these findings [13]; for the observables under consideration, these singularities in solutions from Bethe ansatz turned out to be spurious, eventually.

It was found that both, at zero and at finite temperature the perturbative expansion of the local Green's function for the $d$-electrons converges in the intermediate- to strong-coupling regime $\frac{U}{\pi \Gamma} \geq 2[65 ; 66]$. Values $\frac{U}{\pi \Gamma} \geq 2[63]$ or, depending on the set of considered observables and the desired extent of scale separation, still larger values of $U$, may be regarded as quasi-infinite: most physical quantities are essentially equal to the corresponding values in the Kondo model, in this regime. The weak-coupling regime, $\frac{U}{\pi \Gamma} \leq 1$, is delimited by the breakdown of the Hartree-Fock approximation [6]. The temperature must be $T<T_{K}$ in order to study the strongly correlated regime, because the Kondo resonance is increasingly suppressed, otherwise. The universal scaling properties of the Kondo resonance are correctly reproduced with the quantum Monte Carlo method [67]. Fixed-finite-order perturbation theory in $U$ eventually yields only algebraic scaling of the Kondo temperature in $U$, whereas the correct scaling is given by equation (2.7) [6]. Hence, the speed of convergence is lowered as $U$ is increased or $T$ is decreased. The behaviour of the average expansion order in a Monte-Carlo sampling of the diagrams is related to this (see chapter 4).

Due to its presumably well-behaved convergence properties, an important application of the perturbative expansion in $U$ turned out to be the derivation of exact relations, for finite temperature as well as ground-state properties [6].

\section{Physical Significance}

Systematic studies clarified many aspects of the physics of the equilibrium model even for the strong-coupling limit, and the model is considered to be well-understood in equilibrium nowadays. However, understanding the emergence of the new energy scale $T_{K}$ in detail was a non-trivial task that lasted for several decades. Other aspects such as the spatial realization of the Kondo screening cloud are subject to 
experimental and theoretical investigations, using for example scanning tunneling microscopy [68].

Due to the minimal local structure ${ }^{3}$ already including physically highly non-trivial physics and having a well-understood equilibrium limit, the Anderson model is widely used as a test ground for various computational methods computing transport of electrons through nanostructures in the strongly correlated regime [69]. Understanding the Kondo effect out of equilibrium may be seen as a fundamental key ingredient to understanding quantum transport for this larger class of systems [41]. This and further advance in nanotechnology lead to a "revival of Kondo physics" [70]. Furthermore, the Anderson model is included in the simplest non-trivial realization of the now widely used dynamical mean-field theory (DMFT) for the treatment of the Hubbard model which is for example used to study high- $T_{C}$ superconductivity [71].

\subsubsection{Linear Response Theory}

In order to have a first glance at nonequilibrium, a natural choice is the dissipationfluctuation theorem of statistical mechanics. It is widely and successfully used to study transport characteristics of strongly correlated nanostructures by using equilibrium methods.

The theorem asserts that introducing an infinitesimal perturbation $\lambda(t)$ to an equilibrium system, the response can be calculated from equilibrium correlation functions [72]. The perturbation $\lambda(t)=\theta(t) \tilde{\lambda}(t)$ is switched on at time $t=0$, contributing to the Schrödinger representation of the total Hamiltonian via an operator $O_{\lambda}$,

$$
H_{\lambda}(t)=H+\lambda(t) \cdot O_{\lambda}
$$

During the time evolution, eigenstates of $H$ are perturbed by $\lambda(t)$. It can be shown [72] that the response of an expectation value of an observable $M$,

$$
\left.\frac{\delta\langle M\rangle\left(t_{2}\right)}{\delta \lambda\left(t_{1}\right)}\right|_{\lambda \equiv 0}=-\mathrm{i} \theta\left(t_{2}-t_{1}\right)\left\langle\left[M^{(H)}\left(t_{2}\right), O_{\lambda}^{(H)}\left(t_{1}\right)\right]\right\rangle_{\text {therm }} .
$$

Operators labeled by $(\ldots)^{(H)}$ are Heisenberg representations of the respective quantities in the unperturbed system. The expectation value $\langle\cdots\rangle$ is taken with respect to the $\lambda$-driven out-of-equilibrium statistical ensemble, and $\langle\cdots\rangle_{\text {therm }}$ represents the thermal expectation value.

The linear conductance of an interacting mesoscopic system is derived from this relation [73]. It may be obtained by integrating over the conductivity of the sample, i.e. the local response to an external electrical field.

Unfortunately, the regime in which linear-response is valid, is rather small as compared to intrinsic energy scales for systems which inhere a Kondo resonance: linear

\footnotetext{
${ }^{3}$ I.e. only a single spin-degenerate orbital.
} 
transport characteristics are proportional to the height of the Kondo resonance. A finite bias voltage introduces decoherence effects which may be expected to destroy the resonance as the voltage approaches the order of magnitude of the Kondo temperature.

\subsection{Nonequilibrium Perturbation Theory}

In order to go beyond linear response theory, one has to take expectation values with respect to truely nonequilibrium statistical ensembles. The precise definition of the nonequilibrium ensemble in a formal way is often a cumbersome issue. One of the most important tools used to accomplish this is the Schwinger-Keldysh nonequilibrium perturbation theory [74]. The fundamental principle of setting up the Schwinger-Keldysh theory is starting with a known density operator $\rho_{0}$ at time 0 and then turning on a control parameter in the Hamiltonian which lets the density matrix of the system evolve into the steady-state density matrix.

\subsubsection{Fundamental correlation functions}

Correlators of field operators constitute the fundamental building blocks of any diagrammatic approach to quantum field theory. Considering the initial density operator $\rho_{0}$ for a fermionic system we would, for example, like to compute a simple nonequilibrium steady-state static expectation value such as the density

$$
n(k):=\lim _{t \rightarrow \infty} \frac{\operatorname{Tr} \rho_{0} \psi^{\dagger}(k, t) \psi(k, t)}{\operatorname{Tr} \rho_{0}}
$$

where $k, k^{\prime}$ are some degrees of freedom of the fermionic field operators $\psi(k, t)$ in the Heisenberg picture with respect to full Hamiltonian

$$
H(t)=H_{0}+H_{1}(t) \theta(t)
$$

and $H_{0}$ is the system Hamiltonian for $t<0$, and $\theta(t)$ is the Heaviside stepfunction.

The lesser Green's function

$$
G^{<}\left(k, t ; k^{\prime}, t^{\prime}\right)=-\mathrm{i} \frac{\operatorname{Tr} \rho_{0} \psi^{\dagger}\left(k^{\prime}, t^{\prime}\right) \psi(k, t)}{\operatorname{Tr} \rho_{0}},
$$

certainly contains more than enough information to compute

$$
n(k)=\lim _{t \rightarrow \infty} \mathrm{i} G^{<}(k, t ; k, t) .
$$

Similarly, one may express certain current densities by equal-time values of $G^{<}[74]$. 


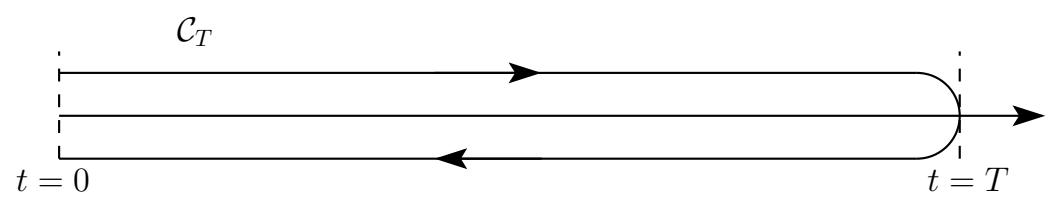

Figure 2.2: The finite-time Keldysh contour $\mathcal{C}_{T}$. Both, the upper and lower part of the contour are located infinitesimally below the real axis in the complex plane for convergence reasons.

The lesser Green's function nevertheless does not contain enough information in order to build up a systematic diagrammatic expansion for expectation values, even in equilibrium statistical mechanics. Here, the time-ordered Green's function

$$
G\left(k, t ; k^{\prime}, t^{\prime}\right)=-\mathrm{i}\left\langle\mathcal{T}\left[\psi(x, t) \psi^{\dagger}\left(x^{\prime}, t^{\prime}\right)\right]\right\rangle_{0},
$$

where $\langle\cdot\rangle_{0}=\operatorname{Tr} \rho_{0} \cdot / \operatorname{Tr} \rho_{0}$, must be considered there, because in perturbation theory, the time-ordered exponential of the time evolution operator in the interaction picture is expanded, while the density operator is treated as the imaginary time evolution from 0 to $-\mathrm{i} \beta$.

Similarly, in the general nonequilibrium situation it is impossible to build a diagrammatic perturbation theory with respect to the regularly time-ordered Green's functions. Instead one has to use a more informative Green's function by setting up a time-ordered perturbation theory on the so-called Schwinger-Keldysh contour $\mathcal{C}_{T}$ which is depicted in figure 2.2. On the upper contour one considers time-ordered operator sequences, on the lower contour anti-time-ordered sequences. The necessity of the contour-ordering for a closed perturbation theory can, again, be seen by formally transforming Green's function expressions between Heisenberg and interaction picture and collecting term in the time-ordered exponential expansions [74].

The central quantity of nonequilibrium perturbation theory is thus the contourordered Green's function

$$
G\left(k, t, \gamma ; k^{\prime}, t^{\prime}, \gamma^{\prime}\right)=-\mathrm{i} \frac{\operatorname{Tr} \rho_{0} \mathcal{T}_{\mathcal{C}_{T}}\left(\psi(k, \gamma, t) \psi^{\dagger}\left(k^{\prime}, \gamma^{\prime}, t^{\prime}\right)\right)}{\operatorname{Tr} \rho_{0}},
$$

where $\gamma, \gamma^{\prime}$ are the Keldysh indices, denoting the upper and lower part of the contour (denoted by - and + , respectively), and $0 \leq t, t^{\prime} \leq T$. Writing the contour-ordered Green's function as a matrix with respect to the Keldysh indices, the diagrammatic perturbation theory is obtained, including a Dyson equation, etc. 


\subsubsection{Application to Nonequilibrium Kondo Systems}

The assumption that unwanted initial correlations die out is by no means trivial in the context of strongly correlated mesoscopic transport and is subject to ongoing investigations, because one has to show that the impurity models are no oversimplification, even by means of purely qualitative nonequilibrium Kondo physics [75]. ${ }^{4}$

While present-day understanding [6] indicates that the equilibrium Kondo effect may be described perturbatively by means of a $U / V$ expansion, one might argue that this is not necessarily helpful in the nonequilibrium case, because the establishment of the nonequilibrium in case of a voltage source a priori involves a direct switching of $V$, which is nonperturbative if $U \neq 0$ and, in contrast to equilibrium theory, involves taking the open-quantum-system limit first (see section 3.1.2). In the Matsubara voltage formalism (and a certain version of Hershfield's formalism, see, e.g. section 3.3), $V$ is switched at $U=0$ and after the establishment of the well-understood non-interacting steady-state, a perturbative expansion with respect to $U / V$ is performed. However, one might argue that the nonequilibrium would have to be introduced directly by a $V / U$ switching, where nonperturbative effects might arise, which otherwise are not taken into account. Such conceptual questions are at present still a matter of debate.

\subsection{Summary}

In this chapter, the Kondo effect in quantum dot systems was introduced. The theoretical description by means of a single-impurity Anderson model was discussed. The theoretical properties of the model and its historical importance were sketched. The formal definition of an out-of-equilibrium density operator by means of Keldysh real-time theory was given. Furthermore, perturbative approaches were discussed by means of their applicability to the nonperturbative Kondo effect.

The next chapter will connect to the Keldysh perturbative real-time approach by first setting up a theoretical framework based on scattering theory. The cumbersome expansion along a long real-time branch is replaced by an imaginary-time formalism which comes at the price of introducing an imaginary-voltage parameter. A perturbative argument is used to connect real and imaginary-time theories.

\footnotetext{
${ }^{4}$ Another application of the Keldysh approach are quantum quenches, i.e. the transient dynamics after turning on an interaction.
} 


\section{The Matsubara-Voltage Formalism}

Apart from the Schwinger-Keldysh perturbation theory, it is also possible to investigate non-equilibrium steady states by means of scattering theory. This is due to the fact that scattering states are (improper) eigenstates of the Hamiltonian of the system under consideration and may serve as a (nearly) complete basis set for a quantum mechanical description. We discuss some important aspects of quantum scattering theory. Based on this, we introduce a Gibbsian approach to steady-state quantum statistical mechanics which was pioneered by Zubarev and applied to mesoscopic systems by Hershfield. The introduction of a Matsubara voltage which makes the latter approach technically more feasible, as proposed by Han and Heary, will be described eventually. It represents the formal basis of the present work.

\subsection{Quantum Theory of Scattering}

\subsubsection{Potential scattering of a single particle}

Many important aspects of the quantum theory of scattering are already contained in the limit of a single nonrelativistic spinless particle which is being scattered by a localized potential $V$ [76]. The Hamiltonian

$$
H=H_{0}+V
$$

acts on the Hilbert space $\mathcal{H}=\ell^{2}\left(\mathbb{R}^{3}\right)$ and has improper eigenstates ${ }^{1}$ and possibly bound states. Plane-wave solutions are the improper eigenstates of $H_{0}=\frac{p^{2}}{2 m}$ and are in a certain manner related to the improper eigenstates of $H$, because the potential $V$ is local and $H_{0}$ governs the time evolution of wave packets which are far away from the interaction region.

This relationship, which is also named as the "asymptotic condition", may be quantified by the so-called Møller wave operators: [76]

$$
\Omega_{ \pm}:=\lim _{t \rightarrow \mp \infty} U(t)^{\dagger} U^{0}(t)
$$

where $U(t)=\mathrm{e}^{-\mathrm{i} H t}$ and $U^{0}(t)=\mathrm{e}^{-\mathrm{i} H_{0} t}$ are the time evolution operators of the interacting and non-interacting systems, respectively.

\footnotetext{
${ }^{1}$ Improper eigenstates are no elements of the original Hilbert space.
} 


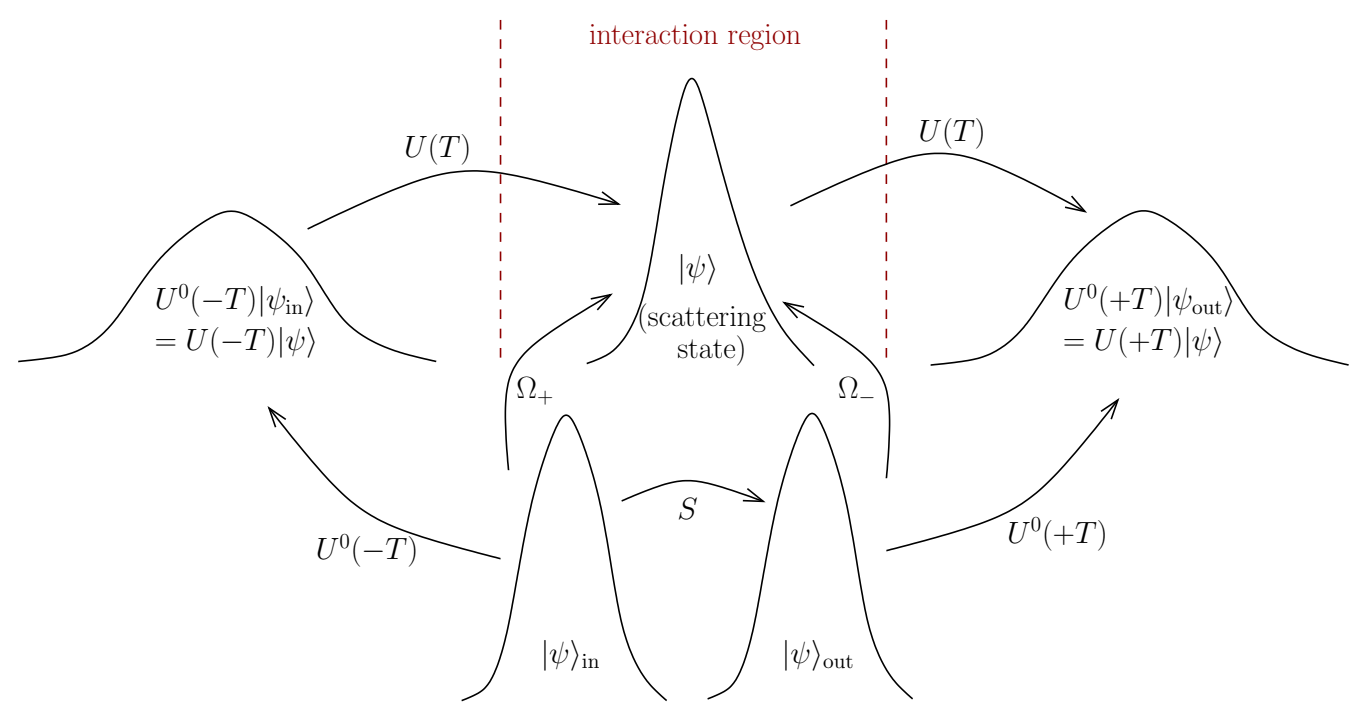

Figure 3.1: One-particle potential scattering process for quasi-infinite times $\pm T, T>0$ in the Schrödinger picture. The in and out asymptotes $U(\mp T)|\psi\rangle$ of the scattering state $|\psi\rangle$ are represented by $\left|\psi_{\text {in }}\right\rangle$ and $\left|\psi_{\text {out }}\right\rangle$, respectively, via the bare time evolution, because the limit $T \rightarrow \infty$ would yield infinitely broadened wave packets being no elements of the Hilbert space $\ell^{2}\left(\mathbb{R}^{3}\right)$. Møller operators $\Omega_{ \pm}$and the $S$-matrix yield the relations between the involved quantities.

\section{Time-dependent scattering states}

The operators $\Omega_{ \pm}$act on $\mathcal{H}$ and also yield results in $\mathcal{H}$. By specifying an arbitrary state $\left|\psi_{\text {in }}\right\rangle \in \mathcal{H}$ the asymptote of the incoming wave packet $U^{0}(t)\left|\psi_{\text {in }}\right\rangle, t \rightarrow-\infty$ should be resembled. The vector $|\psi\rangle=\Omega_{+}\left|\psi_{\text {in }}\right\rangle$ represents the state which this train has at time $t=0$ under the influence of the scattering potential. Similarly, the outgoing wave packet is represented by a state $\left|\psi_{\text {out }}\right\rangle \in \mathcal{H}$ which is related to $|\psi\rangle$ via the second Møller operator, $|\psi\rangle=\Omega_{-}\left|\psi_{\text {out }}\right\rangle$.

Because $|\psi\rangle$ has an in- and an out-asymptote, it is called a scattering state. ${ }^{2}$. The scattering operator

$$
S:=\Omega_{-}^{\dagger} \Omega_{+}
$$

yields the out-asymptote associated to a given in-asymptote of the scattering state $|\psi\rangle$,

$$
\left|\psi_{\text {out }}\right\rangle=S\left|\psi_{\text {in }}\right\rangle
$$

Its matrix elements yield the relevant transition probabilities in scattering experiments.

The action of the present operators is depicted in figure 3.1.

\footnotetext{
${ }^{2}$ In contrast to classical mechanics, it is impossible to find a $|\psi\rangle$ with only an in- but no outasymptote or vice versa [76]
} 


\section{Orthogonality Theorem and Asymptotic Completeness}

In contrast to the scattering states, bound states have no in or out asymptotes. Let $\mathcal{B}$ be the space of bound states, $\mathcal{R}_{ \pm} \subset \mathcal{H}$ the range of the $\Omega_{ \pm}$operators, i.e. the space of scattering states defined by all possible in/out asymptotes $\mathcal{R}_{ \pm}=\Omega_{ \pm} \mathcal{H}$.

The orthogonality theorem states that for sufficiently regular $V$

$$
\begin{array}{lll}
\mathcal{B} & \perp & \mathcal{R}_{-} \\
\mathcal{B} & \perp & \mathcal{R}_{+}
\end{array}
$$

Asymptotic completeness,

$$
\mathcal{R}_{+}=\mathcal{R}_{-}=: \mathcal{R}
$$

may be proved for a wide class of potentials, but is notoriously difficult, in particular in the much more general context of quantum field theory.

Consequently, for an asymptotically complete scattering theory one has

$$
\mathcal{H}=\mathcal{B} \oplus \mathcal{R}
$$

In particular, in the absence of bound states, the scattering states form a basis of the entire Hilbert space. Also the unitarity of $S$ is obtained from asymptotic completeness. In some scattering theories, most prominently the analytic $S$-matrix theory, the unitarity of $S$ is introduced axiomatically.

\section{Stationary scattering states}

While the scattering states $|\psi\rangle \in \mathcal{R}$ have the advantage of being regular vectors of the Hilbert space, their time-dependence is an obstacle for the further development of the theory. In particular, the theory does not refer to the continuous part of the spectrum of $H$ which certainly represents scattering processes but belongs to improper eigenvectors of $H$.

In order to cope with the continuous part of the spectrum one enhances the range of $\Omega_{ \pm}$to improper vectors, namely denoting by

$$
|\vec{p} \pm\rangle:=\Omega_{ \pm}|\vec{p}\rangle
$$

the action of the Møller operators on the plane wave $|\vec{p}\rangle$. While figure 3.1 cannot be literally translated to the $|\vec{p} \pm\rangle$ states, they provide a basis to the $|\psi\rangle \in \mathcal{R}$ which enables an interpretation of the scattering process by means of incoming and outgoing momenta.

Since the Møller operators satisfy the so-called intertwining relation

$$
H \Omega_{ \pm}=\Omega_{ \pm} H_{0}
$$


one finds that the stationary scattering states are in fact improper eigenstates of $H$, with

$$
\begin{aligned}
H_{0}|\vec{p}\rangle & =E_{\vec{p}}|\vec{p}\rangle \\
H|\vec{p} \pm\rangle & =E_{\vec{p}}|\vec{p} \pm\rangle .
\end{aligned}
$$

That is, the continuous parts of the spectra of $H_{0}$ and $H$ are identical. In particular, the $|\vec{p} \pm\rangle$ form an orthonormal basis of $\mathcal{R}$.

Furthermore, one can show that

$$
|\vec{p} \pm\rangle=|\vec{p}\rangle+G\left(E_{\vec{p}} \pm \mathrm{i} 0^{+}\right) V|\vec{p}\rangle,
$$

with the resolvent $G(z)=(z-H)^{-1}$, and

$$
|\vec{p} \pm\rangle=|\vec{p}\rangle+G_{0}\left(E_{\vec{p}} \pm \mathrm{i} 0^{+}\right) V|\vec{p} \pm\rangle,
$$

with the free resolvent $G_{0}=\left(z-H_{0}\right)^{-1}$. Eq. (3.14) is the so-called LippmannSchwinger equation for $|\vec{p} \pm\rangle$. While in one-particle scattering theory with a fixed target, Eq. (3.14) is easier to solve than Eq. (3.13). This advantage disappears in more general scattering theory, so that the term Lippmann-Schwinger equation often synonymously refers to Eq. (3.13). We will also adopt this convention in the following.

\section{Analytic structure}

Apparently, the resolvents $G_{0}(z), G(z)$ have poles on the real axis and are analytic on the upper and lower half plane with respect to $z$. In order to relate the Møller operators to the resolvents for finite values of $\operatorname{Im} z$, one uses the definition

$$
\Omega_{ \pm}=\lim _{\eta \rightarrow \pm 0^{+}} \mp \eta \int_{0}^{\mp \infty} \mathrm{d} t^{\prime} \mathrm{e}^{ \pm \eta t^{\prime}} \mathrm{e}^{\mathrm{i} H t^{\prime}} \mathrm{e}^{-\mathrm{i} H_{0} t^{\prime}}
$$

which is equivalent to (3.2). In addition, for finite $\eta$, the operators

$$
\Omega_{ \pm}^{(\eta)}=\mp \eta \int_{0}^{\mp \infty} \mathrm{d} t^{\prime} \mathrm{e}^{ \pm \eta t^{\prime}} \mathrm{e}^{\mathrm{i} H t^{\prime}} \mathrm{e}^{-\mathrm{i} H_{0} t^{\prime}}
$$

yield the equations

$$
\begin{aligned}
\Omega_{ \pm}^{(\eta)}|\vec{p}\rangle & = \pm \mathrm{i} \eta \cdot G\left(E_{\vec{p}} \pm \mathrm{i} \eta\right)|\vec{p}\rangle \\
& =|\vec{p}\rangle+G\left(E_{\vec{p}} \pm \mathrm{i} \eta\right) V|\vec{p}\rangle
\end{aligned}
$$

Eq. (3.18) makes it more convenient to use Eq. (3.16) for practical calculations and then take $\eta \rightarrow 0^{+}$at the end of the computation. One can derive Eq. (3.18) from (3.17) by using the resummation $G(z)=G_{0}(z)+G(z) V G_{0}(z)$. Taking $\eta \rightarrow 0^{+}$yields 
the Lippmann-Schwinger equation. This procedure was introduced by Gell-Mann and Goldberger [77]. Their physical motivation is that the finite but small $\eta$ averages out unnecessary time-dependent contributions in the definition of the scattering states in combination with the integration with respect to $t^{\prime}$.

\section{Liouvillian Formulation}

In order to apply scattering theory to the steady-state many-body transport problem, a Liouvillian formulation will be useful. Starting with the Liouville-von-Neumann equation for density operators in the Schrödinger picture,

$$
\mathrm{i} \frac{\partial \rho}{\partial t}=[H, \rho]=\mathcal{L} \rho
$$

where $\mathcal{L}=[H, \cdot]$ is the Liouvillian superoperator, rather than the Schrödinger equation $\mathrm{i} \frac{\partial \psi}{\partial t}=H \psi$, one can generalize scattering theory to a Liouvillian formulation for density matrices. In order to translate from Hamiltonian to Liouvillian description in the one-particle problem, one simply has to replace $H$ by $\mathcal{L}=[H, \cdot], V$ by $\mathcal{L}_{V}=[V, \cdot]$, $|\vec{p}\rangle$ by $|\vec{p}\rangle\langle\vec{q}|, E_{\vec{p}}$ by $E_{\vec{p}}-E_{\vec{q}}, H_{0}$ by $\mathcal{L}_{0}=\left[H_{0}, \cdot\right][78]$.

Let us consider the eigenoperators $X_{k}$ of $\mathcal{L}_{0}$ which fulfill the relation

$$
\mathcal{L}_{0} X_{k}=\varepsilon_{k} X_{k}
$$

and yield, using the Møller superoperators

$$
\Omega_{ \pm}^{(\eta)}:=\mp \eta \int_{0}^{\mp \infty} \mathrm{d} t^{\prime} \mathrm{e}^{ \pm \eta t^{\prime}} \mathrm{e}^{\mathrm{i} \mathcal{L} t^{\prime}} \mathrm{e}^{-\mathrm{i} \mathcal{L}_{0} t^{\prime}}
$$

the scattering states

$$
X_{k, \pm}=\Omega_{ \pm}^{(\eta)} X_{k}
$$

Consequently, the Lippmann-Schwinger equation for the scattering states reads

$$
X_{k, \pm}=X_{k}+\frac{1}{\varepsilon_{k} \pm \mathrm{i} \eta-\mathcal{L}} \mathcal{L}_{V} X_{k}
$$

As before, we will denote by

$$
\Omega_{\mp}:=\lim _{\eta \rightarrow 0^{+}} \Omega_{\mp}^{(\eta)}
$$

the actual Møller superoperators. 


\subsubsection{Many-body scattering theory}

The formalism may be generalized straightforwardly to many-body scattering processes. In second quantization, consider the fermionic Hamiltonian

$$
H=\sum_{k} \varepsilon_{k} c_{k}^{\dagger} c_{k}+V
$$

where $V$ may introduce additional degrees of freedom, and $H_{0}=H-V$. Apparently, $\mathcal{L}_{0} c_{k}^{\dagger}=\varepsilon_{k} c_{k}^{\dagger}$ and $\mathcal{L}_{0} c_{k}=-\varepsilon_{k} c_{k}$. Following field operators $\psi_{k, \pm}^{\dagger}$ are therefore obtained from $c_{k}^{\dagger}$ by

$$
\psi_{k, \pm}^{\dagger}:=\Omega_{\mp} c_{k}^{\dagger} .
$$

Note that in order to obtain a similar expression for the adjoint operators $\psi_{k, \pm}$ by means of the annihilators $c_{k}$ one has to flip the sign of the Møller superoperators,

$$
\psi_{k, \pm}=\Omega_{ \pm} c_{k} .
$$

At this point, nothing is known about the (anti-)commutator algebra of the $\psi_{k, \pm}^{(\dagger)}$, however. It might thus be misleading to call them annihilation or creation operators in a general context. Also note that, although the invoked notation is similar to that of the scattering theory of density matrices, we think of $c_{k}^{(\dagger)}$ as field operators in the Heisenberg picture here. Therefore, the roles of $\boldsymbol{\Omega}_{-}$and $\boldsymbol{\Omega}_{+}$are interchanged again, because the time evolution of the field operators is governed by Heisenberg's equation of motion which differs by a sign from the von-Neumann equation which describes the density matrices in the Schrödinger picture: The field operator $c_{k}^{\dagger}$ creates a fermionic excitation in the distant past which evolves to the many-body excitation created by $\boldsymbol{\Omega}_{-} c_{k}^{\dagger}$ under time evolution with respect to $H$.

\section{Infinite Quantum System}

Note that it is crucial that the $k$-sum in Eq. (3.25) has to run over infinitely many fermionic degrees of freedom. Otherwise, one would deal with a finite quantum system. Then, the Poincaré recurrence theorem for finite quantum systems would effectively assert that the interaction term $V$ will not only be acting at time $t \approx 0$ but also at times $t \pm T, t \pm 2 T$, et cetera. A central assumption of scattering theory would thus be violated, making the construction (3.26) meaningless.

In contrast to typical computations for systems in thermal equilibrium it is therefore inherently necessary to take the infinite limit at a very early stage of the formalism: It would be meaningless to compute $\psi_{k, \pm}^{(\dagger)}$ and observables expressed by them in a finite system and then do a finite-size extrapolation. For example, the expectation value of the current operator is always zero in a finite system, due to the recurrence, while it is non-zero for steady-state expectation values of an infinite system. 
A natural starting point to stationary currents of fermions is thus the quantum field theory which emerges from taking an infinite lattice of fermions.

\section{Mathematical Implications}

While an ultraviolet cutoff is inherently imposed by the lattice, still the infinite number of degrees of freedom poses a fundamental problem when included a-priori. It has been pointed out that in principle one might even face certain conceptual limitations of the Fock-space formulation of quantum field theory. ${ }^{3}$ In particular, the existence of a (thermal) density operator is not certain, because one may find counter-examples in similar systems [80]. One has to keep these principal precautions in mind when proceeding with the formalism.

When performing calculations with otherwise ill-defined density operators, one can use the fact that the typical length scale associated to certain recurrence phenomena may be identified. It may be given by $v_{F} t$, where $v_{F}$ is a Fermi velocity. If then the system size $L \gg v_{F} t$, the recurrence phenomenon will not appear. In order to consider certain convergence processes such as the $t \rightarrow \pm \infty$ limits of scattering theory, even more care has to be taken, in order to correctly model the interplay of the remaining relevant energy scales of the system.

\subsection{Hershfield's Operator $Y$}

For the finite-size equilibrium Anderson impurity model one can formally write down the thermal density matrix in the grand canonical ensemble as

$$
\rho_{\text {eq }}:=\mathrm{e}^{-\beta(H-\mu \mathcal{N})},
$$

where $\mathcal{N}$ is the particle-number operator and $\mu$ the chemical potential. Observables may be expressed as $\langle A\rangle=\operatorname{Tr}\left(\rho_{\text {eq }} A\right) / \operatorname{Tr} \rho_{\text {eq }}$, and the thermodynamic limit may be taken at the end of the calculation.

In the steady-state nonequilibrium setup, the situation is not as clear. Hershfield postulated $^{4}$ that the density matrix of the steady-state system effectively may be similarly coped in the Gibbsian form

$$
\rho_{\text {neq }}:=\mathrm{e}^{-\beta(H-\Phi Y)},
$$

where $\Phi$ is the bias voltage $\mu_{L}-\mu_{R}$ and $\beta=\left(k_{B} T\right)^{-1}$ is the temperature of the two leads [29]. The newly appearing operator $Y$ is Hermitian and can be constructed by

\footnotetext{
${ }^{3}$ Consider, e.g., the paradoxa emerging within the field-theoretic limit of BCS theory as discussed in Ref. [79].

4 Other nonequilibrium Gibbsian approaches to quantum transport may already be found in Zubarev's textbook [28].
} 
means of certain scattering states. More generally, having not the bias, but maybe a current as defining parameter of non-equilibrium, Hershfield claims that

$$
\rho_{\text {neq }}:=\mathrm{e}^{-\beta(H-\tilde{Y})},
$$

for some Hermitian operator $\tilde{Y}$.

\subsubsection{Outline of the formalism}

The formal details, such as specifying the appropriate limiting procedures in order to make the involvement of $\rho_{\text {neq }}$ well-defined, with Hershfield's approach are due to the field-theoretic limit somewhat delicate and still subject to intensive ongoing investigations. Hence, we will only discuss the central ideas of the formalism. Details of the appropriate limiting procedure may be found in the literature $[81 ; 82]$. In this work, we will concentrate on the aspects of Hershfield's approach which are also relevant for its imaginary-voltage extension by Han and Heary.

As in Keldysh perturbation theory, one typically defines the non-equilibrium system starting with an equilibrium system at $t=-\infty$. In our case, one can start with zero hopping from the leads into the nanostructure, with the two leads being in thermal equilibrium at different chemical potentials $\mu_{L}$ and $\mu_{R}$, namely with the initial density matrix for the leads,

$$
\rho(t=-\infty):=\mathrm{e}^{-\beta\left(H_{L}-\mu_{L} \mathcal{N}_{L}\right)} \otimes \mathrm{e}^{-\beta\left(H_{R}-\mu_{R} \mathcal{N}_{R}\right)} .
$$

Using the time evolution with the full Hamiltonian of the system, a steady state of the system is approached. For this purpose, the existence of a physical relaxation process is assumed by Hershfield. Doyon and Andrei argue that a formal similarity to Caldeira-Leggett models of quantum decoherence ensures the necessary relaxations automatically within the model $[81 ; 83]$. Nevertheless, the actual approach to and even the mere existence of a steady-state may only be proven for the interacting Anderson impurity model under certain assumptions [75]. Another setup consider a steady-state non-interacting system which may be constructed explicitly either with respect to a certain voltage drop or with respect to a certain current. As the interaction is turned on, the full non-equilibrium problem emerges.

None of these strategies is chosen by Hershfield, but he merely points out that in all of these cases, the initial density matrix already has the form

$$
\rho_{0}=\mathrm{e}^{-\beta\left(H_{0}-\tilde{Y}_{0}\right)} .
$$

Under the assumption that due to the presence of a physical relaxation process the mathematical details work out well, he was able to show that the interacting stationary state may also be written in the above form. In the particular example of the first switching process, namely the two equilibrium reservoirs with chemical 
potentials $\mu_{L}, \mu_{R}$, he finds that

$$
\tilde{Y}=\mu_{R} \sum_{k} \psi_{R, k}^{\dagger} \psi_{R, k}+\mu_{L} \sum_{k} \psi_{L, k}^{\dagger} \psi_{L, k}
$$

Here, $\psi_{\alpha, k}^{(\dagger)}$ operators are the scattering states evolving from the $k$-th band electron $c_{\alpha, k}^{(\dagger)}$ of the lead $\alpha$ via Eq. (3.26). It should again be emphasized that $\psi_{\alpha, k}^{\dagger}$ operators create complicated many-body states involving processes such as particle-hole excitations, etc., and the commutator relations of the $\psi$-operators are a priori unclear.

However, it has recently been shown by Han that

$$
\left\{\psi_{\alpha^{\prime}, k^{\prime}}^{\dagger}, \psi_{\alpha, k}\right\}=\delta_{\alpha, \alpha^{\prime}} \delta_{k, k^{\prime}},
$$

as long as the many-body interaction acts only locally on the nanostructure, so that $\mathcal{L}_{V} c_{\alpha \sigma k}^{\dagger} \propto d_{\sigma}^{\dagger}$, where $\mathcal{L}_{V}$ is the Liouvillian with respect to the hopping and interaction part of the Hamiltonian [84]. Hence, for a large class of mesoscopic systems, including the single-impurity Anderson model, the scattering states are fermions. Han also showed that these fermions provide a complete basis set for the underlying manybody Hilbert space [84].

Note that when we use the common convention of setting the zero of energy to the mean of the two potentials, $\mu_{L}=+\Phi / 2, \mu_{R}=-\Phi / 2$, we have

$$
Y=\sum_{\alpha k} \frac{\alpha}{2} \psi_{\alpha k}^{\dagger} \psi_{\alpha k}
$$

The $Y$ quantum numbers are $\pm 1 / 2$ for a single-electron state $\psi_{ \pm, k}^{\dagger}|0\rangle$ and distinguish the source and drain leads to the nanostructure. In the many-body case the $Y$ quantum number is a measure for the balance of the number of electrons scattering into the nanostructure from source or drain leads, respectively.

\subsubsection{Application of the approach}

The Zubarev-Hershfield approach has the appealing feature of yielding a quasiGibbsian density operator. Nevertheless, it took the scientific community some time to develop the idea further for the following reasons.

First, the benamed mathematical difficulties coming with the introduction of the density operator, have raised concern. Recently, the work by Doyon and Andrei appeared to provide some insight to this topic. One of their results is that one can probably only consider expectation values of "local" observables within Hershfield's formalism, such as the current operator and correlators within the nanostructure.

Second, the definition of the $Y$-operator via interacting scattering states is a highly challenging issue for practical computations. Besides the Lippmann-Schwinger equa- 
tion there is basically no other starting point for a systematic buildup of the scattering states. The scattering-states numerical renormalization group approach [22] imposes a numerically explicit real-time evolution for this purpose. Very recently, Dutt et al. provided a perturbative imaginary-time approach to the Hershfield formalism [82]. It is however only diagrammatic in a sense that the density operator has to be first computed to the desired order via a hierarchy of differential equations, and then the diagrammatic rules define the Green's functions of the nanostructure.

Third, these latter complications are very much due to the fact that the statistical operator and the time evolution operator are no longer closely related. In contrast to this, for thermal Green's functions the Boltzmann factor, using the chemical potential $\mu=0$ as the reference energy, may be written as an evolution from 0 to $-\mathrm{i} \beta$ in imaginary time. The same Hamiltonian is used for the time evolution as in realtime dynamics. This coincidence is heavily used in equilibrium quantum statistical mechanics but is not applicable here.

\subsection{Matsubara-Voltage Approach}

In this section we introduce the imaginary-voltage formalism, which is being investigated in the course of the present thesis. Based on Hershfield's approach, Han and Heary suggested to introduce the following modification of the formalism towards an effective equilibrium, using the Matsubara voltage idea of Fendley et al. [27; 85]. By means of the non-interacting steady-state density matrix,

$$
\rho_{0}=\mathrm{e}^{-\beta\left(H_{0}-\Phi Y_{0}\right)}
$$

they analyzed the Schwinger-Keldysh perturbation theory with respect to $U$. The non-interacting $Y_{0}$ could be explicitly calculated by computing the non-interacting scattering states analytically from the Lippmann-Schwinger equation (3.23), using the respective $\Omega_{-} c_{\alpha k \sigma}^{\dagger}$ states. In the non-interacting Anderson impurity model they obtained:

$$
\left(\psi_{\alpha k \sigma}^{(0)}\right)^{\dagger}=c_{\alpha k \sigma}^{\dagger}-\frac{t_{\alpha}}{\sqrt{\Omega}} g_{d}\left(\varepsilon_{\alpha k}\right) d_{\sigma}^{\dagger}+\sum_{\alpha^{\prime} k^{\prime} \sigma^{\prime}} \frac{t_{\alpha} t_{\alpha^{\prime}}}{\Omega} \frac{g_{d}\left(\varepsilon_{\alpha k}\right)}{\varepsilon_{\alpha k}-\varepsilon_{\alpha^{\prime} k^{\prime}}+\mathrm{i} \eta} c_{\alpha^{\prime} k^{\prime} \sigma^{\prime}}^{\dagger}
$$

for

$$
Y_{0}=\sum_{\alpha k \sigma} \frac{\alpha}{2}\left(\psi_{\alpha k \sigma}^{(0)}\right)^{\dagger} \psi_{\alpha k \sigma}^{(0)},
$$

where $g_{d}$ is the noninteracting Green's function

$$
g_{d, \sigma}(\varepsilon)=\frac{1}{\varepsilon-\varepsilon_{d, \sigma}+\mathrm{i} \Gamma} .
$$


In the next subsections, we will first comment on the structure of interacting expectation values of observables and the principal problem of how to obtain a consistent effective-equilibrium theory from Hershfield's approach. Second, the Matsubara voltages are introduced, and it is argued why they can provide a link between effectiveequilibrium systems and the nonequilibrum system. Third, the effective Hamiltonian is derived, which is easily expressed by means of the noninteracting scattering states and provides the effective-equilibrium systems corresponding to the interacting stationary state.

\subsubsection{Structure of Expectation Values}

Expectation values of static observables with respect to the interacting steady state can be derived by the following:

$$
\langle\hat{A}\rangle=\lim _{T \rightarrow+\infty} \frac{\operatorname{Tr} \hat{A} \mathrm{e}^{-\mathrm{i} \mathcal{L}(T-0)} \rho_{0}}{\operatorname{Tr} \mathrm{e}^{-\mathrm{i} \mathcal{L}(T-0)} \rho_{0}}
$$

where $\mathcal{L}$ is the full Liouvillian and $\rho_{0}$ is the initial density matrix. Using the previously proven [84] fermionic nature of the only implicitly known interacting scattering states $\psi_{\alpha k \sigma}$, and their completeness, the expectation value is formally written by Han and Heary as a functional integral with respect to real-time Grassmann fields representing the $\psi_{\alpha k \sigma}$,

$$
\langle\hat{A}\rangle=\left\langle\int \prod_{\alpha k \sigma}\left(\mathcal{D} \psi_{\alpha k \sigma}^{\dagger} \mathcal{D} \psi_{\alpha k \sigma}\right) A\left(\psi_{\ldots}^{\dagger}(0), \psi_{\ldots}(0)\right) \mathrm{e}^{\mathrm{i} \int L(t) \mathrm{d} t}\right\rangle_{0},
$$

where the Lagrangian is

$$
L(t)=\sum_{\alpha k \sigma} \psi_{\alpha k \sigma}^{\dagger}(t)\left(\mathrm{i} \partial_{t}-\varepsilon_{\alpha k \sigma}\right) \psi_{\alpha k \sigma}(t)
$$

and the diagonality of

$$
H=\sum_{\alpha k \sigma} \varepsilon_{\alpha k \sigma} \psi_{\alpha k \sigma}^{\dagger} \psi_{\alpha k \sigma}
$$

has been used. The expectation value $\langle\cdot\rangle_{0}$ is again taken with respect to the noninteracting steady state $\rho_{0}$.

Aiming at an effective-equilibrium description one should first note that the eigenstates of $H, \psi_{\alpha k \sigma}$ are associated to different chemical potentials $\mu_{L}$ and $\mu_{R}$ via the action of Hershfield's $Y$-operator, as in Eq. (3.33): the density operator is diagonal in the $\psi_{\alpha k \sigma}$-basis and has the form

$$
\rho=\mathrm{e}^{-\beta \sum_{\alpha k \sigma} \psi_{\alpha k \sigma}^{\dagger}\left(\varepsilon_{\alpha k \sigma}-\alpha \Phi / 2\right) \psi_{\alpha k \sigma}} .
$$


The introduction of new eigenvalues $\tilde{\varepsilon}_{\alpha k \sigma}:=\varepsilon_{\alpha k \sigma}-\alpha \Phi / 2$ formally yields the equilibrium density matrix

$$
\rho=\mathrm{e}^{-\beta \sum_{\alpha k \sigma} \psi_{\alpha k \sigma}^{\dagger} \tilde{\varepsilon}_{\alpha k \sigma} \psi_{\alpha k \sigma}}
$$

for the system (3.41) whose dynamics is now characterized by the Lagrangian

$$
L(t)=\sum_{\alpha k \sigma} \psi_{\alpha k \sigma}^{\dagger}(t)\left(\mathrm{i} \partial_{t}-\tilde{\varepsilon}_{\alpha k \sigma}-\alpha \Phi / 2\right) \psi_{\alpha k \sigma}(t) .
$$

Again, the nonzero difference between $\varepsilon_{\alpha k \sigma}$ and $\tilde{\varepsilon}_{\alpha k \sigma}$ corresponds directly to the fact that for nonequilibrium systems, the thermodynamic factor cannot be written as the time evolution operator from 0 to $-\mathrm{i} \beta$ (see section 3.2.2). Nevertheless, the shift is a constant which only depends on the lead index, because the lead electrons stay in thermal equilibrium.

As a next step, in order to obtain a full equilibrium system, the real-time Grassmann fields associated to the interacting scattering states are rescaled as follows:

$$
\psi_{\alpha k \sigma}(t)=\mathrm{e}^{-\mathrm{i} \alpha \Phi t / 2} \tilde{\psi}_{\alpha k \sigma}(t) .
$$

This would remove the $-\alpha \Phi / 2$ term in (3.46). However, both $\psi$ and $\tilde{\psi}$ will not be able to represent equilibrium systems, because the analytic continuation

$$
\mathrm{e}^{-\mathrm{i} \alpha \Phi t / 2} \rightarrow \mathrm{e}^{-\alpha \Phi \tau / 2}
$$

as $t \rightarrow-\mathrm{i} \tau$ is not compatible to the Kubo-Martin-Schwinger (KMS) antiperiodic boundary condition of fermionic Matsubara theory [74; 86; 87].

\subsubsection{Matsubara Voltage Substitution}

In order to solve this dilemma, the Matsubara voltage

$$
\mathrm{i} \varphi_{m}:=\mathrm{i} \frac{4 \pi m}{\beta}, \quad m \in \mathbb{Z}
$$

is introduced. Formally replacing $\Phi$ by $\mathrm{i} \varphi_{m}$ in Eq. (3.47) and in the Lagrangian (3.46) makes both, the formulations with respect to $\psi$ - and $\tilde{\psi}$-fields compatible with Matsubara boundary conditions at once, because the factors $\mathrm{e}^{-\alpha \mathrm{i} \varphi_{m} \tau / 2}$ are $\beta$-periodic with respect to $\tau$. The operation $\psi \rightarrow \tilde{\psi}$ is an equivalent transformation within the functional integral (3.41):

$$
\langle\hat{A}\rangle^{\left(\mathrm{i} \varphi_{m}\right)}=\left\langle\int \prod_{\alpha k \sigma}\left(\mathcal{D} \tilde{\psi}_{\alpha k \sigma}^{\dagger} \mathcal{D} \tilde{\psi}_{\alpha k \sigma}\right) A\left(\tilde{\psi}_{\ldots}^{\dagger}(0), \tilde{\psi}_{\ldots}(0)\right) \mathrm{e}^{-\int_{0}^{\beta} \mathrm{d} \tau \sum_{\alpha k \sigma} \tilde{\psi}_{\alpha k \sigma}^{\dagger}(t)\left(\partial_{\tau}-\tilde{\varepsilon}_{\alpha k \sigma}\right) \tilde{\psi}_{\alpha k \sigma}(t)}\right\rangle_{0} .
$$

Note that this formula implicitly depends on both $\mathrm{i} \varphi_{m}$ and $\Phi$. 
Eq. (3.50) is rather useless by itself. But by reinserting the $\psi$-fields, and by means of the Matsubara voltage substitution applied to the bare band electron energies occuring in the Lagrangian of the original theory,

$$
\varepsilon_{\alpha k \sigma} \rightarrow \varepsilon_{\alpha k \sigma}+\frac{\alpha}{2}\left(\mathrm{i} \varphi_{m}-\Phi\right),
$$

we end up with an equilibrium system which may be described by the Matsubara formalism, because it is equivalent to Eq. (3.50).

\subsubsection{Effective Hamiltonian}

At this point, the system can, like a canonical equilibrium system, again be described by one single non-Hermitian Hamiltonian which appears both, in the statistical and time-evolutional part of the expectation value: For the fully evolved density operator, Eq. (3.44), we have

$$
\rho \equiv \mathrm{e}^{-\beta \sum_{\alpha k \sigma} \psi_{\alpha k \sigma}^{\dagger}\left(\varepsilon_{\alpha k \sigma}+\frac{\alpha}{2}\left(\mathrm{i} \varphi_{m}-\Phi\right)\right) \psi_{\alpha k \sigma}},
$$

because $\mathrm{e}^{-\alpha \beta \varphi_{m} / 2}=1$, and the $\psi_{\alpha k \sigma}^{\dagger} \psi_{\alpha k \sigma}$ operator is just counting 1 or 0 fermions in the scattering state $(\alpha k \sigma)$.

One could use the Hamiltonian $K=H+\left(\mathrm{i} \varphi_{m}-\Phi\right) Y$ as an effective Hamiltonian for the time-evolution and density matrix. Here, however, the problem of not being able to explicitly construct interacting scattering states and thus also not the operator $Y$, reappears. Furthermore, introducing the full density matrix is explicitly avoided in expression (3.40) and in the following theory for that reason. It would not be consequent to use quantities which depend on the interacting scattering states also at this more advanced stage of the formalism.

At first, it is thus important to realize that the point made in the previous subsection can in particular be made for the non-interacting situation $U=0$. In this case, the limiting process (3.40) is trivial. For the initial density operator $\rho_{0}$ we then have

$$
\rho_{0} \equiv \mathrm{e}^{-\beta \sum_{\alpha k \sigma}\left(\psi_{\alpha k \sigma}^{(0)}\right)^{\dagger}\left(\varepsilon_{\alpha k \sigma}+\frac{\alpha}{2}\left(\mathrm{i} \varphi_{m}-\Phi\right)\right) \psi_{\alpha k \sigma}^{(0)}}=: \rho_{0}^{\left(\mathrm{i} \varphi_{m}\right)} .
$$

Yet restricting to the non-interacting case, the effective Hamiltonian is

$$
K_{0}^{\left(\mathrm{i} \varphi_{m}\right)}=\sum_{\alpha k \sigma}\left(\psi_{\alpha k \sigma}^{(0)}\right)^{\dagger}\left(\varepsilon_{\alpha k \sigma}+\frac{\alpha}{2}\left(\mathrm{i} \varphi_{m}-\Phi\right)\right) \psi_{\alpha k \sigma}^{(0)}=H_{0}+\left(\mathrm{i} \varphi_{m}-\Phi\right) Y_{0}
$$

Starting from Hershfield's

$$
\lim _{t \rightarrow \infty} \mathrm{e}^{-\mathrm{i} \mathcal{L}_{H} t} \rho_{0}=\lim _{t \rightarrow \infty} \mathrm{e}^{-\mathrm{i} H t} \rho_{0} \mathrm{e}^{\mathrm{i} H t}=\rho,
$$


Han and Heary now adopt the substitution

$$
K^{\left(\mathrm{i} \varphi_{m}\right)}:=K_{0}^{\left(\mathrm{i} \varphi_{m}\right)}+H_{\mathrm{int}}
$$

as effective Hamiltonian for the Matsubara theory.

Using Eq. (3.56) for the time evolution the quantity

$$
\rho^{\left(\mathrm{i} \varphi_{m}\right)}:=\lim _{t \rightarrow \infty} \exp \left(-\mathrm{i} \mathcal{L}_{K_{0}^{(\mathrm{i} \varphi m)}+H_{\mathrm{int}}} t\right) \rho_{0}^{\left(\mathrm{i} \varphi_{m}\right)} .
$$

can be evaluated within the equilibrium formalism. If an appropriate analytic continuation $\mathrm{i} \varphi_{m} \rightarrow \Phi$ exists, $\rho^{\left(\mathrm{i} \varphi_{m}\right)}$ exactly reduces to the expression (3.55) for $\rho$. The identity $\rho_{0}^{\left(\mathrm{i} \varphi_{m}\right)} \equiv \rho_{0}$, Eq. (3.53), has to be inserted before the analytic continuation is carried out. ${ }^{5}$ Consequently, the Hamiltonian (3.56) can be used for an effective equilibrium calculation, and given the analytic continuation $i \varphi_{m} \rightarrow \Phi$ is adequately possible, the original Hershfield result is reobtained from the Matsubara voltage theory.

\subsection{Physical Observables}

\subsubsection{Range of applicability}

In order to compute physical observables within the Matsubara voltage formalism one first has to check whether and when there is actually an analytic continuation $\mathrm{i} \varphi_{m} \rightarrow \Phi$ which yields physical results, because the formal replacement of $\mathrm{i} \varphi_{m}$ by $\Phi$ in certain terms does not guarantee their existence.

In the original publication, Han and Heary chose to validate their approach for the single-impurity Anderson model. In the wide band limit, the Schwinger-Keldysh perturbative expansion of the dot electron Green's function was compared to the perturbative expansion within the Matsubara formalism. A correct mapping as i $\varphi_{m} \rightarrow \Phi$ of Matsubara theory to real-time theory was indicated by certain term-by-term identifications up to second order in $U$ [27].

\section{Role of $Y_{0}$ quantum number in many-body processes}

In a later publication it was pointed out that under certain circumstances, the correct mapping cannot be realized by a simple analytic continuation rule for higher-order contributions [53]. ${ }^{6}$

\footnotetext{
${ }^{5}$ This rule may preferably be interpreted as a boundary condition to the continuation, as we will see later.

${ }^{6} \mathrm{By}$ an analytic continuation rule we denote a rule to recover real-time theory from a simple functional expression involving certain analytic continuations of the Matsubara theory.
} 


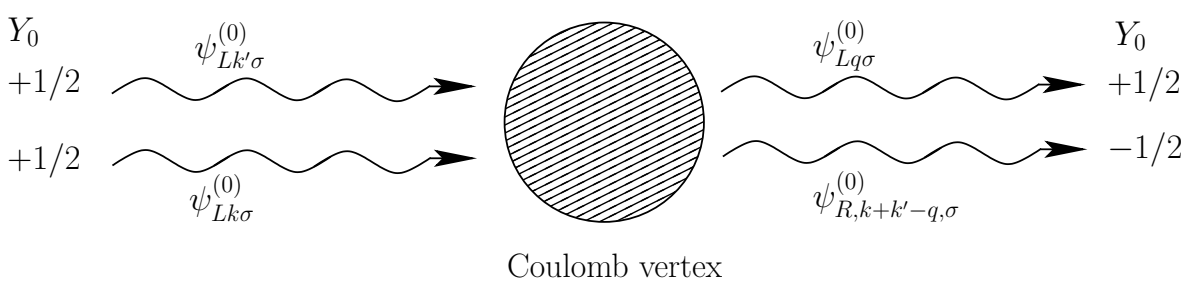

Figure 3.2: A scattering process which does not conserve the $Y_{0}$ quantum number.

The reason for this constraint is a subtlety which itself arises in the first-order perturbation theory with respect to $U$. As sketched in figure 3.2 it is based on the fundamental observation that the $Y_{0}$ quantum number is not conserved by manybody scattering processes: Because the source and drain leads are treated on an equal footing, incoming electrons from the source lead $\left(Y_{0}\right.$ quantum number contribution $+1 / 2$ ) may scatter via the interaction $U$ to the drain lead $\left(Y_{0}\right.$ quantum number contribution $-1 / 2$ ) and vice versa. One may see this formally in the single-impurity Anderson model by rewriting the Coulomb interaction term in the non-interacting scattering states basis: [53]

$$
\begin{aligned}
H_{\mathrm{int}}= & U \sum_{\{\alpha\},\{k\}}\left(\frac{t_{\alpha_{1}} g^{*}\left(\varepsilon_{1}\right)}{\sqrt{\Omega}} \psi_{\alpha_{1} k_{1} \uparrow}^{\dagger}\right)\left(\frac{t_{\alpha_{2}} g\left(\varepsilon_{2}\right)}{\sqrt{\Omega}} \psi_{\alpha_{2} k_{2} \uparrow}\right) . \\
& \cdot\left(\frac{t_{\alpha_{3}} g^{*}\left(\varepsilon_{3}\right)}{\sqrt{\Omega}} \psi_{\alpha_{3} k_{3} \downarrow}^{\dagger}\right)\left(\frac{t_{\alpha_{4}} g\left(\varepsilon_{4}\right)}{\sqrt{\Omega}} \psi_{\alpha_{4} k_{4} \downarrow}\right) .
\end{aligned}
$$

If the source and drain leads are given by the same density of states $\rho(\varepsilon)$, this does not impose a constraint to the applicability of the theory, for symmetry reasons, even for different hoppings $t_{\alpha}$. Nevertheless, the formalism cannot be applied to serially coupled quantum dots [53]. Another serious implication of the missing conservation law for the $Y_{0}$ number is that the imaginary-voltage theory is not applicable to the current operator, although Hershfield's approach is applicable [54; 81]. Nevertheless, the current may often be calculated from the dot Green's function via the MeirWingreen approach [88].

Note that disregarding the formal constraints to Matsubara voltage theory, the fact of the $Y_{0}$ number not being conserved has a simple physical reason. Namely, it is due to the establishment of the correct statistical ensemble for the interacting system. The latter is governed by the interacting $Y$-Operator, via time-evolution with the full Hamiltonian: see the discussion of Eq. (3.57). 
Branch cut at $\operatorname{Im} z_{\varphi}=0$

An additional, but not fundamentally disturbing subtlety is that within certain vertex corrections diagrams appear which introduce a rather involved structure of convoluted bare propagators which gives rise to a branch cut at $\operatorname{Im} z_{\varphi}=0$, where the analytic continuation $\mathrm{i} \varphi_{m} \rightarrow z_{\varphi} \in \mathbb{C}$ has been inserted. Up to present knowledge, the branch cut itself is not a principal constraint, because the physical expectation values may be extracted from the two possible directions approaching it: it is argued that the symmetric averaging over the branch cut generates the correct singular-value type of integrals of the Keldysh real-time expansion [53].

\subsubsection{Structure of the perturbation expansion}

The structure of the imaginary-voltage theory, including some of the previously mentioned properties, may be investigated explicitly in perturbation expansions with respect to $U$ [60-62]. Integrating out the bath degrees of freedom in the wide-band limit, the noninteracting Green's function of the quantum dot becomes [27]

$$
G_{d}^{(0)}\left(\mathrm{i} \varphi_{m} ; \mathrm{i} \omega_{n}\right)=\sum_{\alpha} \frac{\Gamma_{\alpha} / \Gamma}{\mathrm{i} \omega_{n}-\frac{\alpha}{2}\left(\mathrm{i} \varphi_{m}-\Phi\right)-\varepsilon_{d}+\mathrm{i} \Gamma \operatorname{sgn}\left(\omega_{n}-\frac{\alpha}{2} \varphi_{m}\right)} .
$$

In Eq. (3.59), $\Gamma_{\alpha}$ is the hybridization with the lead $\alpha, \Gamma_{\alpha}=\pi N_{F} \cdot\left|t_{\alpha}\right|^{2}$, where $N_{F}$ is the density of states of the respective leads at the Fermi level and $\Gamma=\Gamma_{L}+\Gamma_{R}$. Due to the non-conservation of the quantum number $Y_{0}$ in the interacting theory, we have to assume that $N_{F}$ is identical for source and drain leads, while the hopping amplitudes $t_{\alpha}$ may be different [53].

It is important to underline that no Lehmann representation exists for $G_{0}$, because (3.59) is not analytic with respect to $i \omega_{n}$ on the entire upper half plane $\mathbb{H}[89]$. Further analytic properties will be described in the following chapters.

The time-evolution of the effective Hamiltonian (3.56) is now contained in the time-ordered Matsubara Green's function

$$
G_{d, \sigma}\left(\mathrm{i} \varphi_{m} ; \mathrm{i}\left(\tau-\tau^{\prime}\right)\right)=\int \prod_{\tilde{\sigma}}\left(\mathcal{D}\left[d_{\tilde{\sigma}}, d_{\tilde{\sigma}}^{\dagger}\right]\right) d_{\sigma}(\tau) d_{\sigma}^{\dagger}\left(\tau^{\prime}\right) \mathrm{e}^{-S_{\mathrm{eff}, d}^{\left.\mathrm{i} \varphi_{m}\right)}}
$$

via the $d$-electron quantum effective action

$$
S_{\text {eff }, d}^{\left(\mathrm{i} \varphi_{m}\right)}=\sum_{\tilde{\sigma}} \int_{0}^{\beta} \mathrm{d} \tau \int_{0}^{\beta} \mathrm{d} \tau^{\prime} d_{\tilde{\sigma}}^{\dagger}\left(\tau^{\prime}\right)\left(\mathcal{G}_{d}^{(0)}\right)^{-1}\left(\mathrm{i} \varphi_{m} ; \tau, \tau^{\prime}\right) d_{\tilde{\sigma}}(\tau)+S_{\text {int }}\left[d, d^{\dagger}\right],
$$


where the Hubbard interaction term

$$
S_{\mathrm{int}}=U \cdot \int_{0}^{\beta} \mathrm{d} \tau\left(d_{\downarrow}^{\dagger}(\tau) d_{\downarrow}(\tau)-\frac{1}{2}\right)\left(d_{\uparrow}^{\dagger}(\tau) d_{\uparrow}(\tau)-\frac{1}{2}\right)
$$

represents the local Coulomb repulsion.

In second-order perturbation theory, Han and Heary showed that the particle-hole symmetric irreducible self-energy with respect to the $U$-expansion of (3.60) can be written as

$$
\begin{aligned}
\Sigma^{(2 \mathrm{nd})}\left(\mathrm{i} \varphi_{m} ; \mathrm{i} \omega_{n}\right)= & \sum_{\{\alpha\}} \prod_{i=1,2,3}\left(\int \mathrm{d} \varepsilon_{i} \frac{\Gamma_{\alpha_{i}}}{\Gamma} A_{0}\left(\varepsilon_{i}\right)\right) . \\
& \cdot \frac{f_{\alpha_{1}}\left(1-f_{\alpha_{2}}\right) f_{\alpha_{3}}+\left(1-f_{\alpha_{1}}\right) f_{\alpha_{2}}\left(1-f_{\alpha_{3}}\right)}{\mathrm{i} \omega_{n}-\left(\alpha_{1}-\alpha_{2}+\alpha_{3}\right) \frac{\mathrm{i} \varphi_{m}-\Phi}{2}-\varepsilon_{1}+\varepsilon_{2}-\varepsilon_{3}},
\end{aligned}
$$

where $f_{\alpha_{i}}$ is the Fermi-Dirac function $f\left(\varepsilon_{i}-\alpha_{i} \Phi / 2\right) . \quad A_{0}(\varepsilon)$ is the non-interacting particle-hole symmetric spectral function of the quantum dot, which is given by the Lorentzian distribution

$$
A_{0}(\varepsilon)=\frac{1}{\pi} \frac{\Gamma}{\varepsilon^{2}+\Gamma^{2}}
$$

in the wide-band limit of the Anderson impurity model. One has used the following boundary condition for the Matsubara voltage,

$$
f\left(\varepsilon_{i}+\alpha_{i}\left(\mathrm{i} \varphi_{m}-\Phi\right) / 2\right)=f\left(\varepsilon_{i}-\alpha_{i} \Phi / 2\right) .
$$

Note that apart from the $\alpha$-summations and the energy integrations, the expression (3.63) consists of a single fraction whose numerator is a real function. The denominator has a similar structure to that of the bare Green's function (3.59), but the Matsubara voltage appears in additional ratios $\frac{\alpha_{1}-\alpha_{2}+\alpha_{3}}{2}$ with respect to the Matsubara frequency. In contrast to the bare Green's function, these ratios may also take the values $-3 / 2$ and $+3 / 2$, in addition to $-1 / 2$ and $+1 / 2$ which are already present in $G_{0}$. Similar to equilibrium, the convolution (3.63) leads to discontinuities in the imaginary part of the self-energy which occur whenever the denominator crosses zero.

In general it can be argued $[27 ; 53 ; 90]$ that the higher-order particle-hole excitations result in diagrammatic self-energy contributions, with the approximate structure

$$
D^{\text {(p.h.) }}\left(\mathrm{i} \varphi_{m} ; \mathrm{i} \omega_{n}\right)=\sum_{\gamma \in 2 \mathbb{Z}-1} \int \mathrm{d} \varepsilon \frac{\sigma_{\gamma}\left(\mathrm{i} \varphi_{m} ; \varepsilon\right)}{\mathrm{i} \omega_{n}-\gamma\left(\mathrm{i} \varphi_{m}-\Phi\right) / 2-\varepsilon} .
$$

Here $\sigma_{\gamma}\left(\mathrm{i} \varphi_{m} ; \varepsilon\right)$ are some spectral functions, which are not necessarily real and depend on $\mathrm{i} \varphi_{m}$, including possible non-analyticities with respect to $\mathrm{i} \varphi_{m}$.

Because the particle-hole excitation $D^{\text {(p.h.) }}$ is in general subject to further convo- 
lutions ${ }^{7}$ with certain other terms within the perturbative procedure, it is a priori not clear that $\Sigma$ itself has actual discontinuities along the non-analytic directions of $D^{\text {(p.h.) }}\left(\mathrm{i} \varphi_{m} ; \mathrm{i} \omega_{n}\right)$. Nevertheless, the self-energy and also the Green's function will not be analytic along the directions, i.e. only some higher derivatives of $\Sigma$ and $G$ will actually jump and show discontinuities.

In Ref. [53] it is argued, that also in higher-order contributions to the one-particleirreducible self-energy a systematic partial fraction decomposition of products of energy denominators results in a representation

$$
\Sigma\left(\mathrm{i} \varphi_{m}, \mathrm{i} \omega_{n}\right)=\sum_{\gamma \in 2 \mathbb{Z}-1} \int \mathrm{d} \varepsilon \frac{B_{\gamma}\left(\varepsilon, \mathrm{i} \varphi_{m}-\Phi\right)}{\mathrm{i} \omega_{n}-\frac{\gamma}{2}\left(\mathrm{i} \varphi_{m}-\Phi\right)-\varepsilon}+\Sigma^{(0)}\left(\mathrm{i} \varphi_{m}-\Phi\right)
$$

to become exact, where $B_{\gamma}\left(\varepsilon, \mathrm{i} \varphi_{m}-\Phi\right)$ and the Hartree term $\Sigma^{(0)}\left(\mathrm{i} \varphi_{m}-\Phi\right)$ are analytic as a function of $\mathrm{i} \varphi_{m} \rightarrow z_{\varphi}$ on the upper and lower half plane.

\section{Convolutive Structure of the Feynman graphs}

To make the structure of perturbative expressions more explicit, let us comment in more detail on how an arbitrary one-particle irreducible Feynman diagram of imaginary-voltage theory is structured, using the partial-fraction decomposition argument of Ref. [53]. Without an external magnetic field, a general $N$-th order 1-PI self-energy diagram with external frequency $i \omega_{n}$ has the form

$$
D\left(\mathrm{i} \varphi_{m} ; \mathrm{i} \omega_{n}\right)=\frac{U^{N}}{\beta^{2 N-1}} \sum_{\nu_{1}} \cdots \sum_{\nu_{2 N-1}} \prod_{k=1}^{2 N-1} G_{0}\left(\mathrm{i} \varphi_{m} ; \mathrm{i} \omega_{\nu_{k}}^{(k)}\right) \prod_{l=1}^{N} \delta\left(C_{l}\left(\mathrm{i} \omega_{n} ;\left\{\mathrm{i} \omega_{\nu_{k}}^{(k)}\right\}_{k=1}^{N-1}\right)\right) .
$$

Here, $\delta\left(C_{l}\right)$ is the energy conservation law associated to the $l$-th Coulomb vertex in the diagram. It does not involve the Matsubara voltage $\varphi_{m}$, but is merely a multilinear form with respect to the set of internal and external Matsubara frequencies. The conservation laws now eliminates $N-1$ internal Matsubara frequencies. Without loss of generality, ${ }^{8}$ we can assume that they are the $i \omega_{\nu_{k}}^{(k)}$ with $k>N$. We thus have

$$
\begin{aligned}
D\left(\mathrm{i} \varphi_{m} ; \mathrm{i} \omega_{n}\right)=\left(\frac{U}{\beta}\right)^{N} \sum_{\nu_{1}} \cdots \sum_{\nu_{N}} \prod_{k=1}^{N} G_{0}\left(\mathrm{i} \varphi_{m} ; \mathrm{i} \omega_{\nu_{k}}^{(k)}\right) . \\
\cdot \prod_{k^{\prime}=N+1}^{2 N-1} G_{0}\left(\mathrm{i} \varphi_{m} ; \mathrm{i} \Omega^{\left(k^{\prime}\right)}\left(\mathrm{i} \omega_{n} ;\left\{\mathrm{i} \omega_{\nu_{k}}^{(k)}\right\}_{k=1}^{N}\right)\right) .
\end{aligned}
$$

\footnotetext{
${ }^{7}$ The convolutions are Matsubara summations which can be rewritten using contour integrals.

${ }^{8}$ We can choose the naming convention of the internal Matsubara frequencies in an appropriate way.
} 


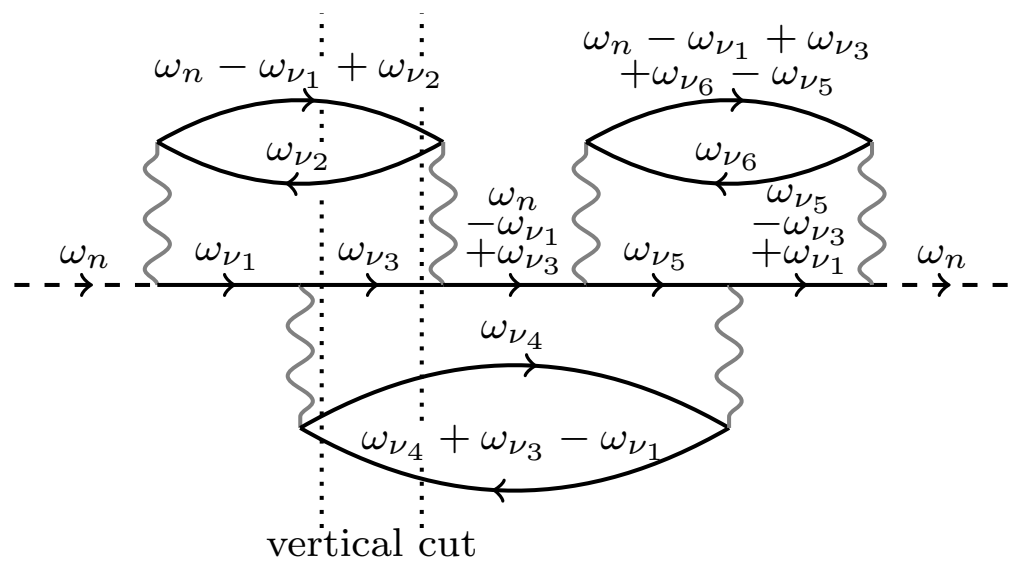

Figure 3.3: An arbitrary 1-PI selfenergy diagram $D\left(i \varphi_{m} ; \mathrm{i} \omega_{n}\right)$ with external Matsubara frequency $i \omega_{n}$ can be written in such a way that $\omega_{n}$ only appears with prefactor 1 or 0 in the internal Matsubara frequencies. One can choose $\omega_{n}$ to appear only in the topmost particlelike propagator for each vertical cut through the diagram. This can be verified inductively by inserting and removing arbitrary pairs of opening/closing vertices in the above diagram. In our notation, the $\Omega^{\left(k^{\prime}\right)}$ functions, $k^{\prime}>6$, are the nontrivial linear combinations of $\omega_{n}$ and the $\omega_{\nu_{k}}$, as presented above.

Here, the $k^{\prime}$-th $\left(k^{\prime}>N\right)$ internal Matsubara frequency is determined by the first $N$ internal frequencies, by defining the linear combination $\Omega^{\left(k^{\prime}\right)}$ according to the conservation law $C_{k^{\prime}-N}$.

It is now crucial to recall that $G_{0}$ is not an analytic function of $i \Omega^{\left(k^{\prime}\right)} \rightarrow z_{\Omega^{\left(k^{\prime}\right)}}$ on the upper and lower half planes, but merely on the stripes and half planes

$$
\begin{aligned}
& \mathbb{S}_{-}=\left\{z_{\Omega^{\left(k^{\prime}\right)}} \in \mathbb{C}\left|\operatorname{Im} z_{\Omega^{\left(k^{\prime}\right)}}<-\right| \varphi_{m} \mid / 2\right\}, \\
& \mathbb{S}_{0}=\left\{z_{\Omega^{\left(k^{\prime}\right)}} \in \mathbb{C}|-| \varphi_{m}\left|/ 2<\operatorname{Im} z_{\Omega^{\left(k^{\prime}\right)}}<+\right| \varphi_{m} \mid / 2\right\}, \\
& \mathbb{S}_{+}=\left\{z_{\Omega^{\left(k^{\prime}\right)}} \in \mathbb{C}|+| \varphi_{m} \mid / 2<\operatorname{Im} z_{\Omega^{\left(k^{\prime}\right)}}\right\} .
\end{aligned}
$$

The mutual boundaries of $\mathbb{S}_{-}$with $\mathbb{S}_{0}$, and of $\mathbb{S}_{0}$ with $\mathbb{S}_{+}$are branch cuts of $G_{0}$ with respect to $z_{\Omega^{\left(k^{\prime}\right)}}$. A Hermitian theory would solely have a branch cut on the real axis.

Within a certain combination of Matsubara indices $n, \nu_{1}, \ldots, \nu_{N}$, the fermionic character of $\Omega^{\left(k^{\prime}\right)}$ enforces the constraint

$$
\Omega^{\left(k^{\prime}\right)}\left(\mathrm{i} \omega_{n} ;\left\{\mathrm{i} \omega_{\nu_{k}}^{(k)}\right\}_{k=1}^{N}\right)=\mu^{\left(k^{\prime}\right)} \cdot \omega_{n}+\sum_{k=1}^{N} \mu_{k}^{\left(k^{\prime}\right)} \cdot \omega_{\nu_{k}}^{(k)},
$$

where the coefficients $\mu^{\left(k^{\prime}\right)}, \mu_{k}^{\left(k^{\prime}\right)} \in\{-1,0,+1\}$ depend only on the bare propagator indices $k^{\prime}$ and $k$, and $\mu^{\left(k^{\prime}\right)}+\sum_{k=1}^{N} \mu_{k}^{\left(k^{\prime}\right)}$ must be an odd number. We can assume that 
the multiplicity of the external energy $\mu^{\left(k^{\prime}\right)}$ only takes the values 0 and 1 . As shown in figure 3.3, one can prove by induction that this choice of representation is not a restriction to generality.

The analytic structure of $G_{0}$ is more explicitly contained in the spectral representation $[53]$

$$
G_{0}\left(\mathrm{i} \varphi_{m} ; \mathrm{i} \omega_{n}\right)=\sum_{\alpha= \pm 1} \int \mathrm{d} \varepsilon \frac{\Gamma_{\alpha}\left|g_{d}(\varepsilon)\right|^{2}}{\mathrm{i} \omega_{n}-\frac{\alpha}{2}\left(\mathrm{i} \varphi_{m}-\Phi\right)-\varepsilon}
$$

where $g_{d}(\varepsilon)$ is defined according to (3.39). The evaluation of Matsubara sums in expression (3.68) can be accomplished similar to a Hermitian equilibrium theory be accomplished by Poisson sum rules. This invokes Fermi-Dirac and Bose-Einstein functions, for which the boundary condition (3.65) of the Matsubara voltage has to be inserted. Since we are interested in the analytic structure with respect to the external frequency, it is insightful to formally rearrange some of the $\left(i \varphi_{m}-\Phi\right)$-terms in Eq. (3.68) first, by a bosonic index shift in the $\nu_{i}$-summations.

We obtain

$$
\begin{aligned}
D\left(\mathrm{i} \varphi_{m} ; \mathrm{i} \omega_{n}\right)= & \left(\frac{U}{\beta}\right)^{N} \sum_{\nu_{1}} \cdots \sum_{\nu_{N}} \prod_{k=1}^{N} \sum_{\alpha_{k}= \pm 1} \frac{\Gamma_{\alpha_{k}} / \Gamma}{\mathrm{i} \omega_{\nu_{k}}^{(k)}+\frac{\alpha_{k}}{2} \Phi-\varepsilon_{d}+\mathrm{i} \Gamma \operatorname{sgn}\left(\omega_{\nu_{k}}\right)} \cdot \\
& \cdot \prod_{k^{\prime}=N+1}^{2 N-1} G_{0}\left(\mathrm{i} \varphi_{m} ; \mathrm{i} \Omega^{\left(k^{\prime}\right)}\left(\mathrm{i} \omega_{n} ;\left\{\mathrm{i} \omega_{\nu_{k}}^{(k)}+\alpha_{k} \mathrm{i} \varphi_{m} / 2\right\}_{k=1}^{N}\right)\right) . \\
= & \left(\frac{U}{\beta}\right)^{N} \sum_{\alpha_{1}= \pm 1} \ldots \sum_{\alpha_{2 N-1}= \pm 1}\left[\prod_{k=1}^{N} \sum_{\nu_{k}} \frac{\Gamma_{\alpha_{k}} / \Gamma}{\mathrm{i} \omega_{\nu_{k}}^{(k)}+\frac{\alpha_{k}}{2} \Phi-\varepsilon_{d}+\mathrm{i} \Gamma \operatorname{sgn}\left(\omega_{\nu_{k}}\right)} .\right. \\
& \left.\cdot \prod_{k^{\prime}=N+1}^{2 N-1} G_{0}^{\left(\alpha_{k^{\prime}}\right)}\left(\mathrm{i} \varphi_{m} ; \mathrm{i} \Omega^{\left(k^{\prime}\right)}\left(\mathrm{i} \omega_{n} ;\left\{\mathrm{i} \omega_{\nu_{k}}^{(k)}+\alpha_{k} \mathrm{i} \varphi_{m} / 2\right\}_{k=1}^{N}\right)\right)\right] .
\end{aligned}
$$

Here, we used the $\alpha_{k^{\prime}}$ th summands of $G_{0}$, namely

$$
G_{0}^{(\alpha)}\left(\mathrm{i} \varphi_{m} ; \mathrm{i} \omega_{n}\right):=\frac{\Gamma_{\alpha} / \Gamma}{\mathrm{i} \omega_{n}-\alpha\left(\mathrm{i} \varphi_{m}-\Phi\right) / 2-\varepsilon_{d}+\mathrm{i} \Gamma \operatorname{sgn}\left(\omega_{n}-\alpha \varphi_{m} / 2\right)},
$$

i.e. $G_{0}\left(\mathrm{i} \varphi_{m} ; \mathrm{i} \omega_{n}\right)=\sum_{\alpha} G_{0}^{\alpha}\left(\mathrm{i} \varphi_{m} ; \mathrm{i} \omega_{n}\right)$. Whenever $\mu^{\left(k^{\prime}\right)}=1$ in (3.73), the external Matsubara frequency is paired with a term $\gamma \mathbf{i} \varphi_{m} / 2$ in $G_{0}^{\left(\alpha_{k^{\prime}}\right)}$, where $\gamma$ is an odd integer. Apart from this, the expression within the squared brackets of (3.76) is formally identical to the output of a Hermitian equilibrium perturbation theory which has a branch cut at $\operatorname{Im} z_{\omega}=0$. This structure is generated by the first term in Eq. (3.67).

In the perturbative expression (3.76) however, the combination of the Matsubara 
frequencies in the denominator of the $G_{0}^{\left(\alpha_{k^{\prime}}\right)}$ in Eq. (3.76) gives rise to

$$
\Omega^{\left(k^{\prime}\right)}-\frac{\alpha_{k^{\prime}}}{2} \varphi_{m}=\left[1 \cdot \omega_{n}+\left(\sum_{k=1}^{N} \mu_{k}^{\left(k^{\prime}\right)} \alpha_{k}-\alpha_{k^{\prime}}\right) \varphi_{m} / 2\right]+\sum_{k=1}^{N} \mu_{k}^{\left(k^{\prime}\right)} \omega_{\nu_{k}}^{(k)} .
$$

This corresponds to different values of $\gamma$ for each factor in $G_{0}^{\left(\alpha_{k^{\prime}}\right)}$ within Eq. (3.76).

We show that these $\gamma$ values are in fact odd numbers. It is important to recall that the energy-conservation coefficients $\mu^{\left(k^{\prime}\right)}$ and $\mu_{k}^{\left(k^{\prime}\right)}$ are constant as functions of the internal Matsubara frequency indices, because they solely balance out the entire diagram with respect to energy conservation. Starting from the definition as the ratio of $\frac{\varphi_{m}}{2}$ and $\omega_{n}$ in each factor $G_{0}^{\left(\alpha_{k^{\prime}}\right)}$ within Eq. (3.76), we obtain

$$
\gamma^{(\{\alpha\},\{\mu\})}=\alpha_{k^{\prime}}-\sum_{k=1}^{N} \mu_{k}^{\left(k^{\prime}\right)} \alpha_{k}
$$

The $\gamma^{(\{\alpha\},\{\mu\})}$ being odd follows from the fermionicity of (3.73) and the fact that $\alpha_{k^{\prime}}$ may take the values 1 and -1 . This suggests the following interpretation: Because there is no conservation law for the $Y_{0}$ quantum number in the Coulomb scattering processes, ${ }^{9}$ the theory consequently produces branch cuts in the 1-PI selfenergy for ratios

$$
\operatorname{Im} z_{\omega}=\frac{\gamma}{2} \operatorname{Im} z_{\varphi}
$$

where $\mathrm{i} \omega_{n} \rightarrow z_{\omega}$ and $\mathrm{i} \varphi_{m} \rightarrow z_{\varphi}$. The fact that we are using ratios of imaginary parts is a result of the convention that the sign function $\operatorname{sgn}\left(\omega_{n}\right)$ analytically continues to $\operatorname{sgn}\left(\operatorname{Im} z_{\omega}\right)$.

\section{Partial Fraction Decomposition}

The locations of the resulting branch cuts are compatible with Eq. (3.67), although it is not yet clear whether there are real discontinuities as branch cuts. As already stated in section 3.4.1, a second kind of branch cut is also generated, which is completely independent of $z_{\omega}$ [53]. In order to see this, the $G_{0}$ functions in Eq. (3.76) have to be re-expressed by means of the representation (3.74). The resulting convoluted product of fractions is given within the brackets of the following expression:

\footnotetext{
${ }^{9}$ See section 3.4.1.
} 


$$
\begin{aligned}
& D\left(\mathrm{i} \varphi_{m} ; \mathrm{i} \omega_{n}\right)=\sum_{\alpha_{1}= \pm 1} \ldots \sum_{\alpha_{2 N-1}= \pm 1} \int \mathrm{d} \varepsilon_{1} \cdots \int \mathrm{d} \varepsilon_{2 N-1} \\
& {\left[\left(\frac{U}{2 \pi \mathrm{i}}\right)^{N} \prod_{k=1}^{N} \int_{\mathcal{C}_{k}} \mathrm{~d} \zeta_{k} \frac{f_{k}\left(\zeta_{k}\right) \Gamma_{\alpha_{k}}\left|g\left(\varepsilon_{k}\right)\right|^{2}}{\zeta_{k}-\varepsilon_{k}} .\right.} \\
& \cdot \prod_{\substack{\left.k^{\prime}=N+1 \\
\left(\mu^{\prime}\right)=1\right)}}^{2 N-1} \frac{\Gamma_{\alpha_{k^{\prime}}}\left|g\left(\varepsilon_{k^{\prime}}\right)\right|^{2}}{\mathrm{i} \omega_{n}-\frac{\gamma^{(\{\alpha\},\{\mu\})}}{2}\left(\mathrm{i} \varphi_{m}-\Phi\right)+\sum_{k} \mu_{k}^{\left(k^{\prime}\right)} \zeta_{k}-\varepsilon_{k^{\prime}}} . \\
& \left.\cdot \prod_{\substack{\left.k^{\prime}=N+1 \\
\left(\mu^{\prime}\right)=0\right)}}^{2 N-1} \frac{\Gamma_{\alpha_{k^{\prime}}}\left|g\left(\varepsilon_{k^{\prime}}\right)\right|^{2}}{\sum_{k} \mu_{k}^{\left(k^{\prime}\right)} \zeta_{k}-\frac{\alpha_{k^{\prime}}}{2}\left(\mathrm{i} \varphi_{m}-\Phi\right)-\varepsilon_{k^{\prime}}}\right] .
\end{aligned}
$$

The function $f_{k}(\zeta)=f\left(\zeta-\alpha_{k} \Phi / 2\right)$ is again the Fermi function, and $\mathcal{C}_{k}$ is an appropriate integration contour for the sum with respect to $i \omega_{\nu_{k}}^{(k)}$.

It is now important to note that the second $k^{\prime}$-product does not depend on $\mathrm{i} \omega_{n}$ but contains singularities with respect to the complexified $i \varphi_{m} \rightarrow z_{\varphi}$. As a next step, one can iteratively employ the partial fraction decompositions

$$
\begin{aligned}
\left(\mathrm{i} \omega_{n}-z_{1}\right)^{-1}\left(\mathrm{i} \omega_{n}-z_{2}\right)^{-1} & =\left(z_{1}-z_{2}\right)^{-1}\left(\left(\mathrm{i} \omega_{n}-z_{1}\right)^{-1}-\left(\mathrm{i} \omega_{n}-z_{2}\right)^{-1}\right) \\
\left(\mathrm{i} \varphi_{m}-z_{1}\right)^{-1}\left(\mathrm{i} \varphi_{m}-z_{2}\right)^{-1} & =\left(z_{1}-z_{2}\right)^{-1}\left(\left(\mathrm{i} \varphi_{m}-z_{1}\right)^{-1}-\left(\mathrm{i} \varphi_{m}-z_{2}\right)^{-1}\right)
\end{aligned}
$$

on Eq. (3.81). One then obtains a structure which may be coped in the form of Eq. (3.67). Eq. (3.67) is thus a general representation of the self-energy. An explicit fourth-order example of this decomposition is demonstrated by Han in the appendix of Ref. [53].

\section{Branch Cut Geometry}

It is now also evident from the perturbative expansion that self-energy and Green's function have branch cuts, which are in fact discontinuities of the functions themselves, with respect to $\mathrm{i} \omega_{n} \rightarrow z_{\omega}$ and $\mathrm{i} \varphi_{m} \rightarrow z_{\varphi}$ for certain values of $\operatorname{Im} z_{\omega}$ and $\operatorname{Im} z_{\varphi}$. These values are specified by the fundamental conditions

$$
\begin{aligned}
\operatorname{Im} z_{\omega} & =\frac{\gamma}{2} \operatorname{Im} z_{\varphi}, \quad \gamma \text { odd } \\
\operatorname{Im} z_{\varphi} & =0
\end{aligned}
$$

Due to its occurrence in second-order perturbation theory, the first kind of branch cuts, Eqs. (3.84), can be thought of being associated with particle-hole excitations. The second kind of branch cut, Eq. (3.85) may be first found in a fourth-order diagram 


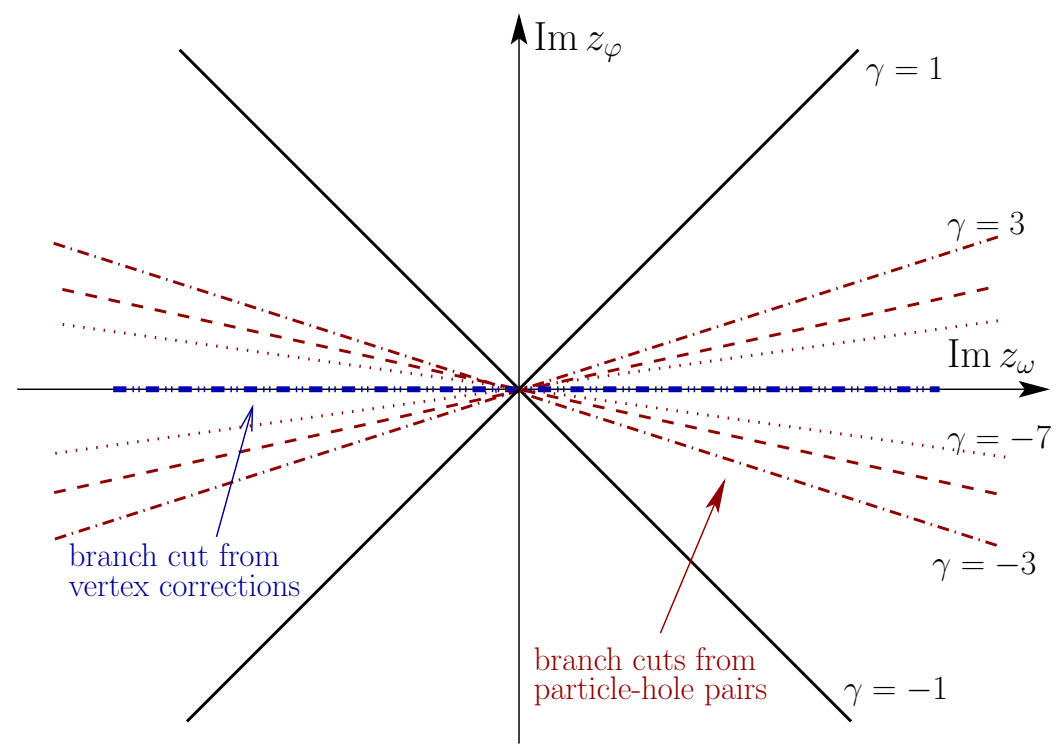

Figure 3.4: Branch cut geometry of dot-electron Green's functions in the Matsubara voltage theory, arising from Eqs. (3.84) and (3.85).

introducing a vertex correction [53]. Note that the strength of the $\gamma$-th branch cut of the (3.84)-kind depends on the highest relevant expansion order $N$ with respect to $U$. Branch cuts for $\gamma \geq N$ are then virtually non-existent, due to Eq. (3.79). It is important to note that physically, the complexity of the branch cut structure is thus proportional to the strength of many-body correlations.

As a more formal consequence from (3.84) and (3.85), a fundamental geometry of Green's functions in the Matsubara voltage theory with respect to the space $\mathbb{C}^{2}$, which is spanned by the pairs $\left(z_{\varphi}, z_{\omega}\right)^{T}$, arises. The geometry is drawn in figure 3.4. It will be the starting point of the systematic function-theoretical framework of chapter 5 .

Following the logic of the preceeding sections, the physical real-time limit of the theory is obtained by first doing the continuation $z_{\varphi} \rightarrow \Phi$ and then doing the continuation $z_{\omega} \rightarrow \omega+\mathrm{i} \delta$. In fact, due to the presence of the (3.85) branch cut, the average of the limits $z_{\varphi} \rightarrow \Phi \pm \mathrm{i} \delta$ has to be taken [53].

\section{Second-Order Approximation to the Continuation}

We continue with a discussion of the representation (3.67) for the selfenergy. A first problem is that nothing is known a priori about the function $B_{\gamma}\left(\mathrm{i} \varphi_{m}, \varepsilon\right)$, except for the existence of the branch cut on the real axis with respect to $i \varphi_{m}$, corresponding to Eq. (3.85). Also, the use of Eq. (3.67) for an analytic continuation is inherently limited by the large number of degrees of freedom which one needs to include in a 
fitting procedure (see chapter 6).

In their original publication, Han and Heary restricted to an approximation to Eq. (3.67) which was motivated by the structure of the second-order selfenergy [27]. Starting from Eq. (3.63) it was required that the particle-hole symmetric self-energy has the following form,

$$
\Sigma\left(\mathrm{i} \varphi_{m} ; \mathrm{i} \omega_{n}\right) \approx \sum_{\gamma \text { odd }} \int \mathrm{d} \varepsilon \frac{\sigma_{\gamma}(\varepsilon)}{\mathrm{i} \omega_{n}-\frac{\gamma}{2}\left(\mathrm{i} \varphi_{m}-\Phi\right)-\varepsilon},
$$

where the spectral functions $\sigma_{\gamma}(\varepsilon)$ are assumed to be real and independent of $i \varphi_{m}$. A fitting procedure with respect to the $\sigma_{\gamma}(\varepsilon)$ guaranteed properly normalized spectral functions through the sum rules which hold for the Dyson equation. Signatures of the nonequilibrium Kondo effect are obtained. In a more recent publication [53], Han made a step towards the representation (3.67) in the numerical fitting procedure by approximating the spectral functions $\sigma$ by a frequency-independent first-order Padé expansion with respect to $\mathrm{i} \varphi_{m}$. However, the approximation (3.86) can only be regarded as a rather uncontrolled way of obtaining results from the Matsubara voltage formalism, because of the ill-posed nature of the actual inverse problem (see chapter 6).

\section{Matsubara Voltage Boundary Condition as Boundedness}

In the course of this thesis it will be important to have a more abstract criterion at hand which is equivalent to the boundary condition of the Green's function with respect to the Matsubara voltage, i.e. identity (3.53) for the density operator. For the Fermi functions in perturbation theory, the boundary condition reads $f\left(\varepsilon-i \varphi_{m}\right)=$ $f(\varepsilon)$. If the condition were not excessively applied to the diagrammatic expansion of the Green's function, the function would diverge as a function of $z_{\varphi} \rightarrow \infty$. Therefore, the boundary condition may simply be interpreted as a statement of boundedness for the two-variable function $G\left(z_{\varphi} ; z_{\omega}\right)$.

\subsubsection{Atomic Limit}

An interesting limiting case of the Matsubara voltage theory is the atomic limit. While it has no apparent physical significance, it provides some insight on how the Matsubara voltage theory itself behaves nonperturbatively. We will find that its analytic structure is consistent with the findings of perturbation theory.

In order to introduce the atomic limit, we set the hybridization to zero in the bare Green's function, namely

$$
G_{d, 0}^{(\text {atom })}=\sum_{\alpha= \pm 1} \frac{1 / 2}{\mathrm{i} \omega_{n}-\frac{\alpha}{2}\left(\mathrm{i} \varphi_{m}-\Phi\right)-\varepsilon_{d}} .
$$




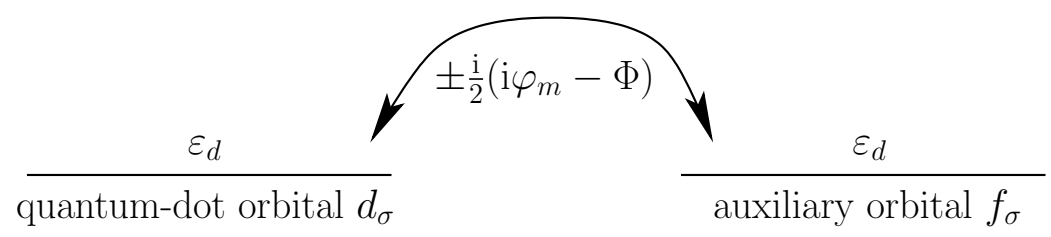

Figure 3.5: The atomic limit $\Gamma=0$ of Matsubara-voltage theory yields a molecule with a non-hermitian hopping of the dot electrons to an auxiliary orbital in the case of formerly symmetric coupling to the source and drain lead leads.

Because in the atomic limit the case of formerly asymmetric coupling to the leads is in fact much more complicated, we restrict to the symmetric one. In this case the imaginary-time Green's operator within the noninteracting part of the effective action (3.61) reduces to the simple expression

$$
\left[\mathcal{G}_{d, 0}^{\text {(atom })}\right]^{-1}=\partial_{\tau}-\varepsilon_{d}-\frac{1}{4} \frac{\left(\mathrm{i} \varphi_{m}-\Phi\right)^{2}}{\partial_{\tau}-\varepsilon_{d}} .
$$

By introducing another pair of spinful fermions $f_{\sigma}$ via a quadratic insertion of Grassmann fields, we equivalently obtain the following simple fermionic molecule: [72]

$$
\begin{aligned}
H_{\mathrm{eff}, \text { atom }}^{\left(\mathrm{i} \varphi_{m}\right)}= & \sum_{\sigma} \varepsilon_{d} d_{\sigma}^{\dagger} d_{\sigma}+U\left(d_{\uparrow}^{\dagger} d_{\uparrow}-\frac{1}{2}\right)\left(d_{\downarrow}^{\dagger} d_{\downarrow}-\frac{1}{2}\right)+ \\
& +\sum_{\sigma} \varepsilon_{d} f_{\sigma}^{\dagger} f_{\sigma}+\sum_{\sigma}\left(-\frac{\mathrm{i}}{2}\left(\mathrm{i} \varphi_{m}-\Phi\right) f_{\sigma}^{\dagger} d_{\sigma}+\frac{\mathrm{i}}{2}\left(\mathrm{i} \varphi_{m}-\Phi\right) d_{\sigma}^{\dagger} f_{\sigma}\right) .
\end{aligned}
$$

It is depicted in figure 3.5.

The auxiliary fermions $f_{\sigma}$ apparently invoke the nonequilibrium statistical ensemble and are decoupled from the dot electrons in the true equilibrium case $i \varphi_{m}-\Phi=0$. Despite being non-Hermitian, the Hamiltonian (3.89) can be diagonalized analytically via a computer algebra system.

Due to the missing availability of a Lehmann representation, the $d$-electron Green's function has to be written using Liouvillians as

$$
\begin{aligned}
G_{d, \sigma}^{(\mathrm{atom})}\left(\mathrm{i} \varphi_{m} ; z_{\omega}\right)= & \operatorname{Tr} \mathrm{e}^{-\beta H_{\text {eff, atom }}^{(\mathrm{i} \varphi m)}} d_{\sigma} \frac{1}{z_{\omega}-\mathcal{L}_{H_{\text {eff, atom }}^{\left(\mathrm{i} \varphi_{m}\right)}}} d_{\sigma}^{\dagger}+ \\
& +\operatorname{Tr} \mathrm{e}^{-\beta H_{\text {eff, atom }}^{(\mathrm{i} \varphi m)}} d_{\sigma}^{\dagger} \frac{1}{z_{\omega}+\mathcal{L}_{H_{\text {eff, atom }}^{\left(\mathrm{i} \varphi_{m}\right)}}} d_{\sigma} .
\end{aligned}
$$




\section{Matsubara Boundary Condition}

For investigating the analytic structure of the Green's function with respect to the variables $\mathrm{i} \varphi_{m} \rightarrow z_{\varphi}$ and $\mathrm{i} \omega_{n} \rightarrow z_{\omega}$ it is now important to ensure that $G_{d, \sigma}^{(\text {atom })}\left(\mathrm{i} \varphi_{m} ; z_{\omega}\right)$ approaches the correct Matsubara boundary condition, by excessively using the identity (3.53) before analytic continuation. The effective-equilibrium density operator $\mathrm{e}^{-\beta H_{\text {eff, atom }}^{\left(\mathrm{i} \varphi_{m}\right)}}$ has some eigenvalues which contain the $\left(\mathrm{i} \varphi_{m}-\Phi\right)$-dependent factors

$$
\begin{aligned}
& \lambda_{1, \pm}=\mathrm{e}^{ \pm \frac{\beta}{4} \sqrt{U^{2}+4\left(\mathrm{i} \varphi_{m}-\Phi\right)^{2}}}, \text { and } \\
& \lambda_{2, \pm}=\mathrm{e}^{ \pm \frac{\beta}{4} \sqrt{U^{2}+16\left(\mathrm{i} \varphi_{m}-\Phi\right)^{2}}} .
\end{aligned}
$$

In contrast to the perturbative expressions from the preceeding section, at finite $U$ it is not clear how the Matsubara boundary condition (3.53) should be imposed on $\lambda_{1, \pm}$ and $\lambda_{2, \pm}$. We will therefore concentrate on the limit $U \rightarrow 0$ in the following. As we will see, this limit is in fact nontrivial, because $U / \Gamma$ is the relevant interaction scale for the Kondo effect, and $\Gamma$ had already been set to zero in the beginning of the calculation.

\section{Poles of the atomic Green's function}

In the limit $U \rightarrow 0$, poles of the properly analytically continued Green's function occur at the points

$$
\begin{aligned}
& z_{\omega}=\varepsilon_{d} \pm \frac{1}{2}\left(z_{\varphi}-\Phi\right), \text { or } \\
& z_{\omega}=\varepsilon_{d} \pm \frac{3}{2}\left(z_{\varphi}-\Phi\right)
\end{aligned}
$$

with respect to $z_{\omega}$. The values coincide with some eigenvalues of the Liouvillian due to the resolvent structure of expression (3.90).

With respect to $\mathrm{i} \varphi_{m} \rightarrow z_{\varphi}$, a singularity is obtained at the value

$$
z_{\varphi}=\Phi
$$

It emerges from the diagonalization procedure with respect to the Hamiltonian. We see that taking the limit $z_{\varphi} \rightarrow \Phi$ essentially involves dealing with a singular structure.

\section{Comparison to Perturbation Theory}

Obviously, the singularities (3.93) and (3.94) are located on the branch cuts of the first kind, Eq. (3.84). Similarly, the singularity (3.95) is located on the second kind of branch cut, (3.85). One may thus interpret the $(\gamma= \pm 3, \pm 1)$-branch cuts and also the ( $\left.\operatorname{Im} z_{\varphi}=0\right)$-branch cut as $U \rightarrow 0$ poles from the atomic limit, as being smeared 
out by the hybridization to the leads. The limit $z_{\varphi} \rightarrow \Phi$ in both variants is nontrivial due to the presence of a singularity. The singularity has to be averaged out in order to obtain the correct physical result, $z_{\varphi} \rightarrow \Phi \pm \mathrm{i} \delta$.

We have also learned that in contrast to the first kind, the second kind of branch cuts cannot simply be extracted from eigenvalues of the Hamiltonian. It is rather an intrinsic feature of the diagonalization procedure with respect to the effective Hamiltonian. This involves the analytic structure of the corresponding eigenvectors. Its analogue in the perturbative approach is the vertex correction.

\subsubsection{Symmetry Relations}

It is straightforward to see that $G_{0}$ complies with the symmetry relation

$$
G_{0}\left(-\mathrm{i} \varphi_{m} ;-\mathrm{i} \omega_{n}\right)^{*}=G_{0}\left(\mathrm{i} \varphi_{m} ; \mathrm{i} \omega_{n}\right)
$$

in general. Inspection of the perturbation series (3.75) then proves that the same symmetry relation holds for the full Green's function and self-energy.

Similarly, using a symmetric coupling to the leads, $\Gamma_{L}=\Gamma_{R}$, one has an invariance of $G_{0}$ with respect to $\left(\mathrm{i} \varphi_{m}-\Phi\right) \leftrightarrow-\left(\mathrm{i} \varphi_{m}-\Phi\right)$. In expression (3.81) one does immediately see that this invariance is also present in full Green's function and selfenergy.

\subsubsection{Analytic Structure of Static Observables}

In the imaginary-time theory, expectation values of static observables may be written as

$$
\langle A\rangle=\frac{\operatorname{Tr} \mathrm{e}^{-\beta K^{(\mathrm{i} \varphi m)}} A}{\operatorname{Tr} \mathrm{e}^{-\beta K^{(\mathrm{i} \varphi m)}}},
$$

for which one has the expansion [54]

$$
\begin{aligned}
\operatorname{Tr} \mathrm{e}^{-\beta K^{\left(\mathrm{i} \varphi_{m}\right)} A=} & \operatorname{Tr} A \rho_{0}+\operatorname{Tr} A \frac{1}{-\mathcal{L}_{\left.K_{0}^{(\mathrm{i} \varphi m}\right)}} \mathcal{L}_{V} \rho_{0}+ \\
& +\operatorname{Tr} A \frac{1}{-\mathcal{L}_{K_{0}^{\left(\mathrm{i} \varphi_{m}\right)}}} \mathcal{L}_{V} \frac{1}{-\mathcal{L}_{K_{0}^{\left(\mathrm{i} \varphi_{m}\right)}}} \mathcal{L}_{V} \rho_{0}+\cdots
\end{aligned}
$$


For example, the second-order contribution to this expansion can be written by means of the noninteracting scattering-states basis as

$$
\begin{aligned}
& \left.\operatorname{Tr} \mathrm{e}^{-\beta K^{\left(\mathrm{i} \varphi_{m}\right)}} A\right|_{2 \text { nd }}=\sum_{\nu \mu \kappa}\left[\frac{\rho_{0, \nu}}{\left(K_{0, \nu}^{\left(\mathrm{i} \varphi_{m}\right)}-K_{0, \mu}^{\left(\mathrm{i} \varphi_{m}\right)}\right)\left(K_{0, \nu}^{\left(\mathrm{i} \varphi_{m}\right)}-K_{0, \kappa}^{\left(\mathrm{i} \varphi_{m}\right)}\right)}+\right. \\
& +\frac{\rho_{0, \mu}}{\left(K_{0, \mu}^{\left(\mathrm{i} \varphi_{m}\right)}-K_{0, \nu}^{\left(\mathrm{i} \varphi_{m}\right)}\right)\left(K_{0, \mu}^{\left(\mathrm{i} \varphi_{m}\right)}-K_{0, \kappa}^{\left(\mathrm{i} \varphi_{m}\right)}\right)}+ \\
& \left.+\frac{\rho_{0, \kappa}}{\left(K_{0, \kappa}^{\left(\mathrm{i} \varphi_{m}\right)}-K_{0, \nu}^{\left(\mathrm{i} \varphi_{m}\right)}\right)\left(K_{0, \kappa}^{\left(\mathrm{i} \varphi_{m}\right)}-K_{0, \mu}^{\left(\mathrm{i} \varphi_{m}\right)}\right)}\right] V_{\nu \mu} V_{\mu \kappa} A_{\kappa \nu} .
\end{aligned}
$$

As $i \varphi_{m}$ is analytically continued to $z_{\varphi}$, the spectrum of $K_{0}^{\left(z_{\varphi}\right)}=H_{0}+\left(z_{\varphi}-\Phi\right) Y_{0}$ will only include zero for $\operatorname{Im} z_{\varphi}=0$. Apart from the terms in the denominator in which the Fock states in the differences such as $\left(K_{0, \nu}^{\left(\mathrm{i} \varphi_{m}\right)}-K_{0, \mu}^{\left(\mathrm{i} \varphi_{m}\right)}\right)$ have the same $Y_{0}$ quantum number, the function (3.99) has poles only on the real axis with respect to $z_{\varphi}$. The terms with identical $Y_{0}$ quantum number are however constant as a function of $z_{\varphi}$. The same will be true for an arbitrary higher-order contribution to $\langle A\rangle\left(z_{\varphi}\right)$.

Therefore, we can expect the quantity $\langle A\rangle\left(z_{\varphi}\right)$ to be holomorphic on the upper and lower half plane with respect to the complexified imaginary voltage $z_{\varphi}$.

\subsection{Summary}

The present chapter dealt with the concept of Hershfield's $Y$-operator, starting from the basic notions of quantum scattering theory. Hershfield's approach has the shortcomings of the nonexistence of an explicit expression for $Y$ and the emergence of two different Hamiltonians for imaginary-time and real-time evolution. The latter point makes a direct application of Matsubara theory very cumbersome. The limitations are overcome by the imaginary-voltage approach by Han and Heary at the cost of an increase in complexity of the analytic structure of physical observables and a smaller set of considerable systems and observables. By means of perturbation theory and a nonperturbative analysis, the analytic structure of observables and correlation functions has been worked out. For the evaluation of the current through an Anderson impurity, a double analytic continuation of the dot-level Green's function has to be performed. This is particularly problematic, because the Green's function exhibits a large amount of branch cuts in the two-dimensional complex space $\left(z_{\varphi}, z_{\omega}\right) \in \mathbb{C}^{2}$.

Chapter 5 will introduce concepts from the function theory of several complex variables and apply them to the unconventional analytic structure of the dot-level Green's function. This connects to chapter 6, where the MaxEnt method is used to infer physical properties from simulation data and to chapter 7 , in which the analytic continuation theory is extended systematically. The next chapter will briefly intro- 
duce continuous-time quantum Monte-Carlo methods and will show that they provide high-quality solutions to the effective actions of the Matsubara-voltage formalism. 


\section{Quantum Monte Carlo}

In this chapter we introduce the Monte Carlo techniques which are used in order to solve the effective-equilibrium quantum impurity systems. We refer to either the Hamiltonian

$$
H=H_{\mathrm{loc}}+H_{\mathrm{hyb}}+H_{\mathrm{bath}}+H_{\mathrm{int}}
$$

or the action-based formulation

$$
S=S_{\mathrm{loc}}+S_{\mathrm{hyb}}+S_{\mathrm{bath}}+S_{\mathrm{int}}
$$

of the Anderson impurity model in thermal equilibrium which was introduced in chapter 2.2. We first give a brief introduction of the algorithms involved. The implementation of the Matsubara voltage approach is discussed. Finally, the computational performance of the algorithms is analyzed.

\subsection{Algorithms}

\subsubsection{Interaction Expansion}

For almost 20 years the standard Monte Carlo quantum impurity solver used to be the Hirsch-Fye method. It was introduced in 1986 by Hirsch and Fye and imposes a Trotter-Suzuki discretization of imaginary time into $M$ equal slices [91]. A HubbardStratonovich transformation is imposed, and a field of Ising spins acts on the now noninteracting electrons. Observables are estimated by an appropriate sampling of the auxiliary field variables. However, the computational complexity of the algorithm increases as a function of $M$. In order to remove systematic artifacts, the extrapolation $M \rightarrow \infty$ has to be carried out rather carefully and binds computational resources.

It turns out [92] that an equivalent method to extrapolating Hirsch-Fye is the continuous-time interaction expansion method by Rubtsov et al. [30], which is nowadays often referred to as "CT-INT". The algorithm is the outcome of a line of continuous-time algorithms starting with bosonic lattice model simulations which had been generalized to fermions [33].

Rubtsov's algorithm is in many aspects equivalent [92] to the "CT-AUX" formulation by Gull et al. [93] which is formally more similar to Hirsch-Fye. The fundamental idea of the algorithms is to divide the Hamiltonian into two parts, $H=H_{a}+H_{b}$ and 
write partition sum and observables in the Dirac picture with respect to $H_{a}$, e.g.

$$
\begin{aligned}
Z & =\operatorname{Tr} \mathcal{T}_{\tau} \mathrm{e}^{-\beta H_{a}} \mathrm{e}^{-\int_{0}^{\beta} \mathrm{d} \tau H_{b}(\tau)} \\
& =\sum_{k}(-1)^{k} \int_{0}^{\beta} \mathrm{d} \tau_{1} \cdots \int_{\tau_{k-1}}^{\beta} \mathrm{d} \tau_{k} \operatorname{Tr} \mathrm{e}^{-\beta H_{a}} H_{b}\left(\tau_{k}\right) \cdots H_{b}\left(\tau_{1}\right) .
\end{aligned}
$$

Continuous-time algorithms enable to sample the $k$-sum and $\tau_{i}$-integrals simultaneously, generating topologically correct diagrams [33].

In the interaction expansion approach, $H_{a}=H_{\mathrm{hyb}}+H_{\mathrm{bath}}+H_{\mathrm{loc}}$. Within our work we impose the auxiliary-field type of CT-QMC for including the interaction. In case of the CT-AUX algorithm it is technically advantageous to introduce an additional term into the Hamiltonian, namely $H_{a} \rightarrow H_{a}+K / \beta$, and $H_{b} \rightarrow H_{b}-K / \beta$, where $K$ is some arbitrary constant number.

Within the auxiliary-field decomposition [94] one finds that [33]

$$
Z=\sum_{k=0}^{\infty} \sum_{s_{1}, \ldots, s_{k}= \pm 1} \int_{0}^{\beta} \mathrm{d} \tau_{1} \cdots \int_{\tau_{k-1}}^{\beta} \mathrm{d} \tau_{k}\left(\frac{K}{2 \beta}\right)^{k} Z_{k}\left(\left\{s_{k}, \tau_{k}\right\}\right)
$$

where

$$
Z_{k}\left(\left\{s_{i}, \tau_{i}\right\}\right)=\operatorname{Tr} \prod_{i=k}^{1} \mathrm{e}^{-\Delta \tau_{i} H_{a}} \mathrm{e}^{s_{i} \gamma\left(n_{\uparrow}-n_{\downarrow}\right)},
$$

$\gamma:=\operatorname{arcosh}\left(1+\frac{U \beta}{2 K}\right)$, and $\Delta \tau_{i}=\tau_{i+1}-\tau_{i}, i<k ; \Delta \tau_{k}=\beta-\tau_{k}+\tau_{1}$. The relative contribution of a configuration $\left(\left\{\tau_{i}\right\}_{i=1}^{k},\left\{s_{i}\right\}_{i=1}^{k}\right)$ to the partition sum simplifies to [93]

$$
\frac{Z_{k}\left(\left\{s_{i}, \tau_{i}\right\}\right)}{Z_{0}}=\operatorname{det} \mathbf{N}_{\uparrow}^{-1}\left(\left\{s_{i}, \tau_{i}\right\}\right) \cdot \operatorname{det} \mathbf{N}_{\downarrow}^{-1}\left(\left\{s_{i}, \tau_{i}\right\}\right)
$$

with

$$
\begin{aligned}
\mathbf{N}_{\sigma}^{-1}\left(\left\{s_{i}, \tau_{i}\right\}\right) & :=\mathrm{e}^{V_{\sigma}^{\left\{s_{i}\right\}}}-\mathbf{G}_{0, \sigma}^{\left\{\tau_{i}\right\}}\left(\mathrm{e}^{V_{\sigma}^{\left\{s_{i}\right\}}}-1\right), \text { where } \\
\mathrm{e}^{V_{\sigma}^{\left\{s_{i}\right\}}} & :=\operatorname{diag}\left(\mathrm{e}^{\gamma(-1)^{\sigma} s_{1}}, \ldots, \mathrm{e}^{\gamma(-1)^{\sigma} s_{k}}\right) .
\end{aligned}
$$

The notations $(-1)^{\uparrow}:=1,(-1)^{\downarrow}:=-1$, and the bare Green's function matrix $\left(\mathbf{G}_{0, \sigma}^{\left\{\tau_{i}\right\}}\right)_{i, j}:=G_{0, \sigma}\left(\tau_{i}-\tau_{j}+0^{+}\right)$have been used. A representation of a Monte-Carlo configuration $\left\{s_{i}, \tau_{i}\right\}$ is depicted in figure 4.1a.

\section{Sign Problem}

The coefficients of the partition function in (4.6) represent weights for the measurement of observables. Because they are not necessarily positive, a probabilistic interpretation for a Monte-Carlo sampling procedure is not readily available. For example, 
the fermionic anticommutator relations generate plus- and minus-signs. In the case of Matsubara-voltage theory, the Hamiltonian is not hermitian and generates complex weight factors. In order to perform a Monte-Carlo average over configurations $\mathcal{C}$ of a quantity

$$
\langle A\rangle=\sum_{\mathcal{C}} w_{\mathcal{C}} A_{\mathcal{C}}
$$

one performs a Monte-Carlo sampling of averages

$$
\langle A\rangle_{|\cdot|}=\sum_{\mathcal{C}}\left|w_{\mathcal{C}}\right| A_{\mathcal{C}}
$$

and $\langle s\rangle_{|\cdot|}$, where $s_{\mathcal{C}}=w_{\mathcal{C}} /\left|w_{\mathcal{C}}\right|$ is the phase factor of the weight. Then, the actual observable is given by

$$
\langle A\rangle=\frac{\langle A\rangle_{|\cdot|}}{\langle s\rangle_{|\cdot|}}
$$

If the average sign $\langle s\rangle_{|\cdot|}$ is smaller than the error of its estimate (this is usually the case for comparably small $\left.\left|\langle s\rangle_{|\cdot|}\right|\right)$, the uncertainty of the $\langle A\rangle$ estimate is extremely large.

While within the single-impurity Anderson model, the sign problem may be completely eliminated by choosing an appropriate value of $K$, [31] the fermionic sign problem is supposed to be $N P$-hard within more complex models [95].

\subsubsection{Hybridization Expansion}

A complementary approach to CT-AUX is the CT-HYB solver by Werner et al. [96]. The expansion of the partition sum is performed with respect to the hybridization of the quantum impurity and the conduction band degrees of freedom. For density-density like interactions and diagonal hybridization to the bath, as in the single-impurity Anderson model, a simple representation of the partition sum can be derived. One finds [33]

$$
\begin{aligned}
& Z=Z_{\text {bath }} \prod_{\sigma} \sum_{k_{\sigma}=0}^{\infty} \int_{0}^{\beta} \mathrm{d} \tau_{1}^{\sigma} \cdots \int_{0}^{\beta} \mathrm{d}{\tau^{\prime}}_{k_{\sigma}}^{\sigma} . \\
& \cdot \operatorname{Tr}_{d}\left[\mathcal{I}_{\tau} \mathrm{e}^{-\beta H_{\mathrm{loc}}} d_{\sigma}\left(\tau_{k_{\sigma}}^{\sigma}\right) d_{\sigma}^{\dagger}\left({\tau^{\prime}}_{k_{\sigma}}^{\sigma}\right) \cdots d_{\sigma}\left(\tau_{1}^{\sigma}\right) d_{\sigma}^{\dagger}\left({\tau^{\prime}}_{1}^{\prime \sigma}\right)\right] \operatorname{det} \Delta_{\sigma},
\end{aligned}
$$

with the hybridization function

$$
\Delta_{\sigma}(\tau)=\sum_{p} \frac{\left|V_{p}\right|^{2}}{\mathrm{e}^{\beta \varepsilon_{p}}+1} \begin{cases}-\mathrm{e}^{-\varepsilon_{p}(\tau-\beta)}, & \text { if } 0<\tau<\beta \\ -\mathrm{e}^{-\varepsilon_{p} \tau}, & \text { if }-\beta<\tau<0 .\end{cases}
$$




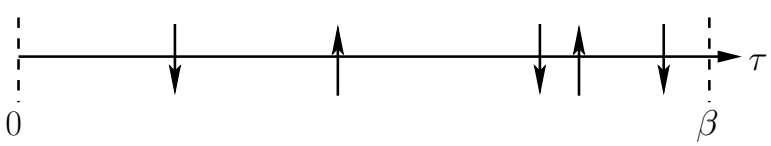

(a) CT-AUX algorithm

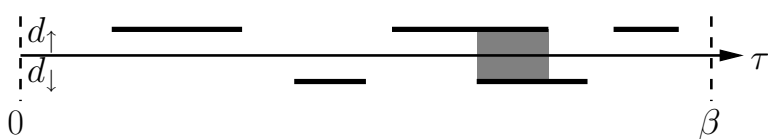

(b) CT-HYB algorithm

Figure 4.1: Configurations within the continuous-time quantum Monte-Carlo algorithms.

The trace over local degrees of freedom can be represented by so-called segments spanned by the pairs of creation and annihilation operators $d_{\uparrow}^{(\dagger)}$ and $d_{\downarrow}^{(\dagger)}$ which were introduced by the density-density interaction. A Monte-Carlo configuration is given by a set of segments, as depicted in figure $4.1 \mathrm{~b}$ which can be pictorially interpreted as spin-up or -down electrons living for a certain lifetime on the imaginary-time interval. Whenever there is an overlap between spin-up and -down segments occurs, the Coulomb interaction introduces a penalty term proportional to $\mathrm{e}^{-U \cdot \Delta \tau}$, where $\Delta \tau$ is the length of the overlap.

\subsection{Application to Matsubara-Voltage Formalism}

\subsubsection{CT-AUX}

The implementation of the $i \varphi_{m}$-th effective-equilibrium effective action of Matsubara voltage theory is straightforward. At a given value of $i \varphi_{m}$, the imaginary-time noninteracting Green's function serves as input to the quantum impurity solver. As opposed to the Hirsch-Fye algorithm, the noninteracting Green's function is resolved arbitrarily well. This is particularly important, because in contrast to conventional Matsubara Green's functions, for rising $\varphi_{m}$, the imaginary-time Green's function develops increasingly severe oscillations, see the lower panel of figure 4.2. Since it is desirable to be able to compute a large range of $\varphi_{m}$ quantities associated to a given physical expectation value for analytic continuation purposes, thus using strongly oscillating $G_{0}(\tau)$ the usage of continuous-time algorithms is practically inevitable.

Practically, in order to introduce very large values of $\varphi_{m}$, it is most convenient to factorize out the oscillating parts of $G_{0}(\tau)$ and introduce them directly into the 


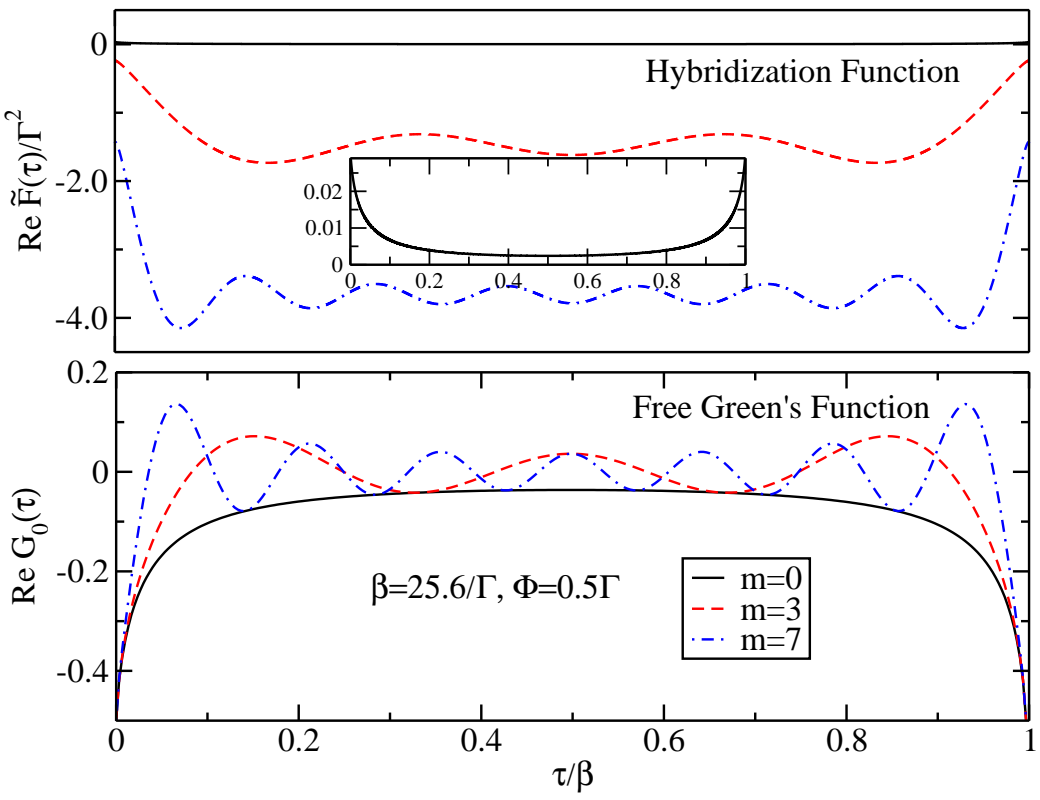

Figure 4.2: Imaginary-Time $G_{0}$ and modification of hybridization function within Matsubara-voltage theory.

quantum Monte-Carlo code. If the frequency-space bare Green's function is given by

$$
\begin{aligned}
G_{0, \sigma}\left(\mathrm{i} \omega_{n}\right) & =\sum_{\alpha= \pm 1} \frac{1}{2} G_{0, \sigma, \alpha}^{\left(\mathrm{i} \varphi_{m}-\Phi\right)}\left(\mathrm{i} \omega_{n}\right), \text { with } \\
G_{0, \sigma, \alpha}^{\left(\mathrm{i} \varphi_{m}-\Phi\right)}\left(\mathrm{i} \omega_{n}\right) & :=\frac{1}{\mathrm{i} \omega_{n}-\frac{\alpha}{2}\left(\mathrm{i} \varphi_{m}-\Phi\right)-\varepsilon_{d, \sigma}+\mathrm{i} \Gamma \operatorname{sgn}\left(\omega_{n}-\frac{\alpha}{2} \varphi_{m}\right)},
\end{aligned}
$$

one can factor out the oscillations by writing

$$
G_{0, \sigma}(\mathrm{i} \tau)=\frac{1}{2} \sum_{\alpha= \pm 1} \mathrm{e}^{-\frac{\alpha}{2} \mathrm{i} \varphi_{m} \tau} f_{\alpha, \varepsilon_{d, \sigma}}(\tau)
$$

where a high-precision tabulation of the regular factor

$$
f_{\alpha, \varepsilon_{d, \sigma}}(\tau)=\frac{1}{\beta} \sum_{\omega_{n}} \mathrm{e}^{-\mathrm{i} \omega_{n} \tau} \frac{1}{\mathrm{i} \omega_{n}+\frac{\alpha}{2} \Phi-\varepsilon_{d, \sigma}+\mathrm{i} \Gamma \operatorname{sgn}\left(\omega_{n}\right)}
$$

can be performed within the initialization procedure of the simulation. A direct tabulation of $G_{0, \sigma}(\mathrm{i} \tau)$ is, even for very fine imaginary-time grids, too inaccurate for large values of $\varphi_{m}$. 


\subsubsection{CT-HYB}

In order to apply the CT-HYB solver to the Matsubara-voltage formalism we have to refuge to the action-based formulation of the algorithm [96]. The hybridization function $F\left(\mathrm{i} \omega_{n}\right)$ is obtained from the bare Green's function via Dyson's equation,

$$
F\left(-\mathrm{i} \omega_{n}\right)=\mathrm{i} \omega_{n}-\varepsilon_{d}-G_{0}\left(\mathrm{i} \varphi_{m} ; \mathrm{i} \omega_{n}\right)^{-1}
$$

Straightforward algebraic manipulation yields

$$
\begin{aligned}
F\left(\mathrm{i} \omega_{n}\right)= & \frac{1}{\mathrm{i} \omega_{n}-\varepsilon_{d}+\frac{\mathrm{i} \Gamma}{2} \sum_{\alpha= \pm 1} \operatorname{sgn}\left(\omega_{n}-\frac{\alpha}{2} \varphi_{m}\right)}\left(\prod_{\alpha= \pm 1}\left[\mathrm{i} \Gamma \operatorname{sgn}\left(\omega_{n}-\frac{\alpha}{2} \varphi_{m}\right)\right]+\right. \\
& \left.+\sum_{\alpha= \pm 1}\left[\mathrm{i} \omega_{n}-\varepsilon_{d}+\alpha\left(\mathrm{i} \varphi_{m}-\Phi\right)\right] \frac{\mathrm{i} \Gamma}{2} \operatorname{sgn}\left(\omega_{n}-\frac{\alpha}{2} \varphi_{m}\right)-\left(\frac{\mathrm{i} \varphi_{m}-\Phi}{2}\right)^{2}\right) .
\end{aligned}
$$

Care has to be taken because of the expressions i $\Gamma \operatorname{sgn}\left(\omega_{n}-\alpha \varphi_{m} / 2\right)$, which are due to the wide-band limit, while for the CT-HYB solver the expansion order diverges. It is therefore necessary to replace the signum function by a sufficiently well-behaved function corresponding to a finite band width.

The high-frequency behaviour of (4.20) is given by

$$
\begin{aligned}
F\left(\mathrm{i} \omega_{n}\right) & \stackrel{\left|\omega_{n}\right| \rightarrow \infty}{\rightarrow} \sum_{\alpha= \pm 1} \frac{\mathrm{i} \Gamma}{2} \operatorname{sgn}\left(\omega_{n}-\frac{\alpha}{2} \varphi_{m}\right)+\frac{c_{1}}{\mathrm{i} \omega_{n}}=: \hat{F}\left(\mathrm{i} \omega_{n}\right)+\frac{c_{1}}{\mathrm{i} \omega_{n}}, \text { with } \\
c_{1} & =-\Gamma^{2}-\left(\frac{\mathrm{i} \varphi_{m}-\Phi}{2}\right)^{2} .
\end{aligned}
$$

In order to perform the Fourier transform of the hybridization function, we separate off the regular contribution

$$
\Delta F(\tau):=\frac{1}{\beta} \sum_{n=-\infty}^{\infty}\left[F\left(\mathrm{i} \omega_{n}\right)-\left(\hat{F}\left(\mathrm{i} \omega_{n}\right)+\frac{c_{1}}{\mathrm{i} \omega_{n}}\right)\right] \mathrm{e}^{-\mathrm{i} \omega_{n} \tau} .
$$

The irregular leading order high-frequency tail $c_{1} /\left(\mathrm{i} \omega_{n}\right)$ yields a constant shift $-c_{1} / 2$ in $F(\tau), 0<\tau<\beta$. The term

$$
\hat{F}\left(\mathrm{i} \omega_{n}\right):=\sum_{\alpha= \pm 1} \frac{\mathrm{i} \Gamma}{2} \operatorname{sgn}\left(\omega_{n}-\alpha \frac{\varphi_{m}}{2}\right)
$$


on the other hand yields

$$
\hat{F}(\tau)=\frac{1}{\beta} \sum_{n=-\infty}^{\infty} \hat{F}\left(\mathrm{i} \omega_{n}\right) \mathrm{e}^{-\mathrm{i} \omega_{n} \tau}=\frac{\Gamma}{\beta} \frac{\cos \left(\varphi_{m} \tau / 2\right)}{\sin (\pi \tau / \beta)} .
$$

Eq. (4.24) diverges for $\tau \rightarrow 0$ and $\tau \rightarrow \beta$, because of the wide-band limit and causes the expansion order of the algorithm to diverge. As a consequence, in practical computations, one can introduce a cutoff parameter $F_{\text {cut }}$ and use

$$
F(\tau)=\Delta F(\tau)-\frac{c_{1}}{2}+\min \left(\hat{F}(\tau), F_{\text {cut }}\right) .
$$

In practice, we used a value $F_{\text {cut }}=10^{4} \Gamma^{2}$, and $\Delta F(\tau)$ was obtained by accumulating the series numerically. In order to illustrate the difference to the conventional implementation to the hermitian single-impurity Anderson Hamiltonian, we investigate the quantity

$$
\tilde{F}(\tau)=F^{\mathrm{eq}}(\tau)-\hat{F}(\tau),
$$

where $F^{\mathrm{eq}}(\tau)=\frac{\Gamma}{\beta}(\sin (\tau / \beta))^{-1}$ is the standard equilibrium hybridization function. A plot is shown in the upper panel of figure 4.2.

\subsection{Computational Performance}

The computational performance of the algorithms drastically depends on the sign or phase problem if present and the average expansion order. When applied to the imaginary-voltage approach, a phase problem is observed for both, the CT-AUX and the CT-HYB algorithms.

We find that in accordance with the literature [31; 96] there is no sign problem present in the hermitian limit $i \varphi_{m}=0$ of the effective impurity model. However, as demonstrated in figure 4.3, there is an increasingly severe phase problem obtained for both, the CT-AUX and the CT-HYB. Nevertheless, there is a drastic difference in computational performance at increasing values of $i \varphi_{m}$. While the CT-HYB approach experiences an exponentially growing phase problem, the problem appears to be much milder for CT-AUX. The severity for CT-HYB is due to the quadratically increasing shift $c_{1}$, see Eq. (4.21), towards negative values in $F(\tau)$ as a function of $i \varphi_{m}$, as in Eq. (4.25). CT-HYB is thus limited to high-precision computations of small $\mathrm{i} \varphi_{m}$ at large values of $U / \Gamma$.

The CT-AUX solver, nevertheless, is able to provide unbiased high-quality data in the Kondo regime over a large regime of the Matsubara voltage variable. In order to demonstrate this, data for the Matsubara self-energy at $U=10 \Gamma, \Phi=0.018 \Gamma$, $T / \Gamma=0.0098$ and $\varphi_{m}=0(m=0), \varphi_{m} / \Gamma=1.23(m=10), \varphi_{m} / \Gamma=2.46(m=$ $20)$, and $\varphi_{m} / \Gamma=3.69(m=30)$ are plotted in figure 4.4. The equilibrium Kondo 


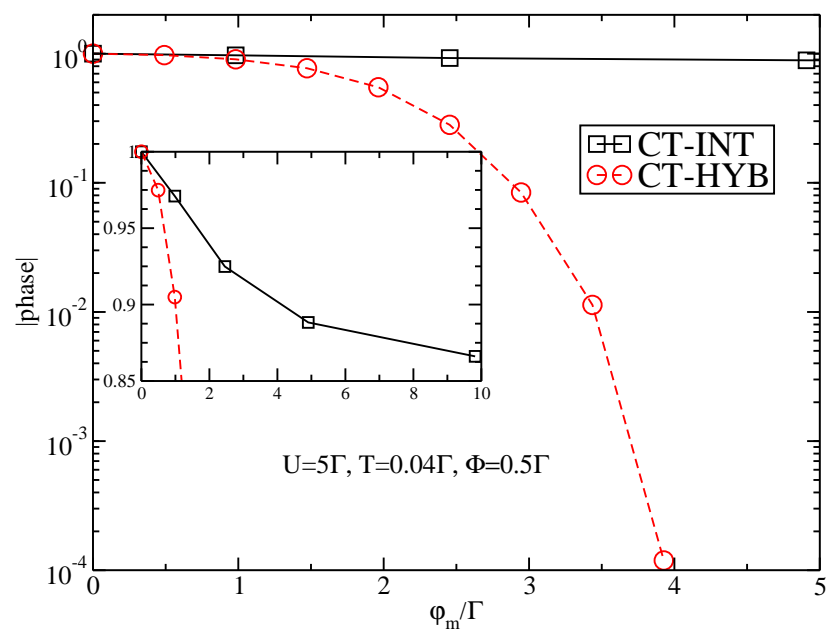

Figure 4.3: Absolute values of the average sampling weight phase factors $\mathrm{e}^{\mathrm{i} \gamma}$ of the CTAUX (solid lines) and the CT-HYB (dashed lines) solver, respectively, as a function of the imaginary voltage. On a logarithmic scale, the average phase decays faster than linearly for the CT-HYB approach when $\varphi_{m}$ is increased. No strong dependence on $\varphi_{m}$ is found for the CT-AUX algorithm.

temperature for this parameter set is $T_{\mathrm{K}} / \Gamma \approx 0.018 \ll 1$, i.e. we are reasonably deep in the Kondo regime of the Anderson model. In sharp contrast to Hirsch-Fye results presented in Ref. [27] we do not observe any discontinuities of the self-energy with respect to the Matsubara frequency. Note that based on this evidence, a recipe has been proposed to alleviate this issue within Hirsch-Fye in reference [53].

\subsection{Summary}

A brief introduction to the continuous-time quantum Monte-Carlo algorithms was given. It was shown that they are particularly suited for solving the effectiveequilibrium systems defined by the Matsubara-voltage formalism. The latter gives rise to a set of effective-equilibrium Green's functions which have a strongly oscillatory behaviour on the imaginary time axis that in contrast to the Hirsch-Fye algorithm can be resolved arbitrarily well by CT-QMC. We found that the CT-AUX algorithm is able to provide highly precise effective-equilibrium data in the non-equilibrium Kondo regime over a broad range of Matsubara voltages. The latter is required for an adequate analytic continuation with respect to $\mathrm{i} \varphi_{m}$. The CT-HYB solver was found to be limited to small values of $\varphi_{m}$ due to a severe sign problem which makes it less useful for practical computations within the Matsubara-voltage approach.

The numerical analytic continuation of data obtained with the CT-QMC approach will be done with the MaxEnt methods developed in chapters 6 and 7 . In order 


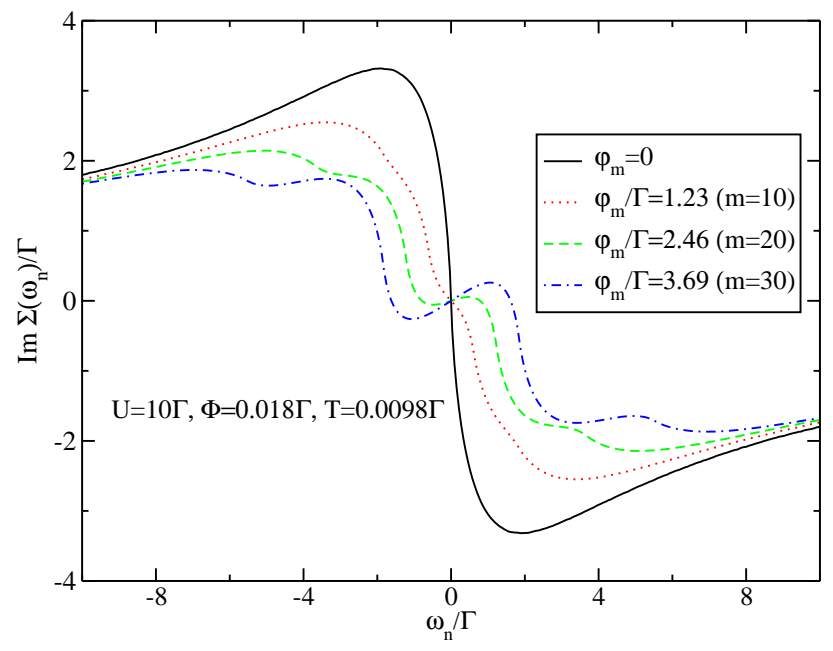

Figure 4.4: Imaginary part of the impurity self-energy obtained with the CT-AUX solver for $V_{G}=0, U / \Gamma=10, T / \Gamma=0.0098$ and $\Phi / \Gamma=0.018$. The equilibrium Kondo scale here is $T_{\mathrm{K}} / \Gamma \approx 0.018$. We easily obtain high-quality data for all values $m=10,20$ and 30 of the complexified voltage, even in this most challenging parameter regime $T_{\mathrm{K}} \ll \Gamma, \Phi \approx T_{\mathrm{K}}$ and $T \approx T_{\mathrm{K}} / 2$. Each $m$-value was run on a single Intel Xeon(R) E5345 CPU for approx. 24 hours, so the data were obtained with relatively moderate computational effort.

to provide necessary mathematical foundations for the analytic continuation of the dot-electron Green's function, the next chapter will issue an introduction to the function theory of several complex variables. Apart from fundamental insights about the structure of the Green's function, it will provide the integral relations between imaginary-time and real-time structure which are necessary for an extraction of dynamical quantities from the QMC data. 
4. Quantum Monte Carlo 


\section{Dynamic Expectation Values and Several Complex Variables}

Regarding dynamic observables, computing physical expectation values from Matsubara voltage simulation data involves both, an analytic continuation with respect to the Matsubara voltage $i \varphi_{m} \rightarrow z_{\varphi} \in \mathbb{C}$ and the fermionic Matsubara frequency $\mathrm{i} \omega_{n} \rightarrow z_{\omega} \in \mathbb{C}$. It implies that a function $G(\underline{z}), \underline{z}:=\left(z_{\varphi}, z_{\omega}\right)^{T} \in \mathbb{C}^{2}$, in particular its reconstruction from a certain set of values, must be studied. This unconventional situation requires tools from the theory of analytic functions of several complex variables.

In this chapter, a detailed introduction to the mathematical structures of several complex variables which are necessary for a profound understanding is provided. In particular, similarities with and differences to one-variable complex analysis are pointed out. As a next step, dynamic correlation functions $G\left(z_{\varphi}, z_{\omega}\right)$ are studied within the resulting theory. Uniqueness of the mathematical procedure involved in the analytical continuations is proved and the connection to maximum entropy approaches for the inference of spectral functions is made (cf. chapter 6). The latter subject connects to the next chapter, in which the numerical implementation is discussed.

Without the presence of $i \varphi_{m}$, i.e. within conventional Matsubara theory, Green's functions are analytic on the upper and lower half-planes, $\mathbb{H}$ and $\mathbb{H}^{*}$. Due to the $\frac{1}{z_{\omega}}$ decay of $G\left(z_{\omega}\right)$ as $z_{\omega} \rightarrow \infty$, a Lehmann spectral representation with respect to the real axis is used [97]. The branch cut on the real axis represents the only set of points $z_{0} \in \mathbb{C}$ for which the Cauchy-Riemann differential equations

$$
\left.\frac{\partial G}{\partial \bar{z}_{\omega}}\right|_{z_{0}}=0
$$

do not hold. ${ }^{1}$ The very location of the branch cut gives rise to the spectral representation in Matsubara theory, i.e. it yields, due the nonsingular structure at $\infty$, a unique characterization of the analytic structure [98].

In order to discuss the holomorphy in the several complex variables context we will partially follow the book on single-sheeted domains of holomorphy, by Vladimirov [99].

\footnotetext{
${ }^{1}$ For notational convenience, we introduced the Wirtinger derivative $\frac{\partial}{\partial \bar{z}}:=\frac{\partial}{\partial x}+\mathrm{i} \frac{\partial}{\partial y}$, with $z=x+\mathrm{i} y$; $x, y \in \mathbb{R}$.
} 
A function $G$ of one complex variable is holomorphic at a point $z$, if and only if condition (5.1) is satisfied. A natural extension of this definition is given for holomorphy of functions of several complex variables. A function $f(\underline{z}), \underline{z} \in \mathbb{C}^{d}$ is holomorphic with respect to $\underline{z}$ at the point $\underline{z}^{(0)}$ if and only if it is holomorphic with respect to each individual variable,

$$
\left.\frac{\partial f}{\partial \bar{z}_{i}}\right|_{\underline{z}^{(0)}}=0, \quad i=1, \ldots, d .
$$

Hartogs' fundamental theorem ${ }^{2}$ asserts that this definition is in fact equivalent to the Weierstraß definition of holomorphy. The latter calls a function holomorphic at $\underline{z}_{0}$ if and only if there exists an open neighbourhood $M$ of $\underline{z}^{(0)}$, such that for all $\underline{z} \in M$ the function $f$ may be written as an absolutely convergent power series $f(\underline{z})=$ $\sum_{|\alpha|=0}^{\infty} a_{\alpha}\left(\underline{z}-\underline{z}^{(0)}\right)^{\alpha} . \alpha$ denotes the multiindex for the monomial $\underline{z}^{\alpha}:=\prod_{n=0}^{d} z_{n}^{\alpha_{n}}$, and $|\alpha|:=\sum_{n=0}^{d} \alpha_{n}$. An analytic complex function of several variables is holomorphic.

The notion of holomorphy implied by Eqs. (5.2) is thus as natural and intuitive as in the one-dimensional case.

\subsection{Domains of Holomorphy and Biholomorphic Transformations}

The major important qualitative difference of single- and multivariable complex analysis is, however, the notion of a domain, and the geometric equivalences among holomorphic functions arising from classes of domains. The notion of a domain in single-variable complex analysis is replaced by the notion of a domain of holomorphy in multi-variable complex analysis - the notion of a conformal map is replaced by the notion of a biholomorphic map. We will address the most prominent differences by first reminding the reader of basic structural properties of one-dimensional complex analysis and then introducing the corresponing terminologies of the more-dimensional theory.

\subsubsection{One-dimensional function theory}

In one-dimensional complex analysis, domains are defined as open connected subsets of $\mathbb{C}$. We will for the first moment restrict to simply connected open sets, i.e. open connected sets with no holes. Conformal maps between domains $U, V$, namely functions

$$
m: U \rightarrow V, \quad z \mapsto m(z)
$$

\footnotetext{
${ }^{2}$ See chapter VII, $\S \S 2,3$ in Ref. [100] or chapter I, $\S 2.6$ in Ref. [101] or chapter I, section 4.2 in Ref. [99].
} 
which are holomorphic and invertible (one-to-one), provide links between certain classes of domains. The Riemann mapping theorem ${ }^{3}$ states that conformal maps between any simply connected $U \neq \mathbb{C}, \emptyset$ and the unit disk exist. I.e. all simply connected domains $\neq \mathbb{C}, \emptyset$ are conformally equivalent: their structures of holomorphic functions map one-to-one to each other and are conformally diffeomorphic. Generalizing the concept of holomorphy to Riemannian surfaces, conformal maps exist only for surfaces of the same topological genus. ${ }^{4}$ The Poincaré-Koebe uniformization theorem $^{5}$ finds that for simply connected Riemann surfaces (topological genus 0), up to conformal equivalence, three classes exist:

- the unit disk $D_{1}=\{z \in \mathbb{C}:|z|<1\}$,

- the complex plane $\mathbb{C}$,

- and the Riemann sphere $\mathbb{C} \cup\{\infty\}$.

These three form the so-called moduli space of genus 0, defined as the space of conformally inequivalent Riemann surfaces of genus 0 . In contrast, the moduli space of $g=1$ is infinite. In general, the moduli space of a Riemann surface of genus $g$ gains complexity as a function of $g$. Each modulus represents an equivalence class of holomorphic functions. ${ }^{6}$

Conformal equivalence plays an important role in physical applications such as two-dimensional potential flows around airfoils or conformal field theory, because it allows for many powerful simplifications. One natural consequence of the conformal equivalence of all non-empty simply connected domains $U \subsetneq \mathbb{C}^{1}$ is that there always exists a function which is not analytically continuable beyond the domain: the function

$$
f_{0}(z)=\sum_{\alpha=0}^{\infty} z^{\alpha !}
$$

is holomorphic on the unit disk but may not be analytically continued to larger domains [99]. Using a conformal map $m$ from the unit disk to $U$, which exists due to the Riemann mapping theorem, one finds the function $f_{0} \circ \mathrm{m}^{-1}$ which cannot be analytically continued beyond $U$. One also calls the unit disk the domain of holomorphy of $f_{0}$, i.e. the largest domain for which $f_{0}$ is holomorphic. Similarly, $U$ is the domain of holomorphy of $f_{0} \circ \mathrm{m}^{-1}$. If there exists any function $f$ for which a domain is a domain of holomorphy, the domain is called a domain of holomorphy.

\footnotetext{
${ }^{3}$ See chapter VII, $\S 4$ in Ref. [102].

${ }^{4}$ The topological genus is in simple words the number of holes of an orientable Riemannian surface. The notion also exists for non-orientable surfaces. For further information see the brief article by Hirzebruch and Kreck [103].

${ }^{5}$ See theorem $10-3$ in Ref. [104].

${ }^{6}$ An intuitively accessible discussion of these findings is provided by Roger Penrose in chapter 8 of Ref. [105].
} 
In general, any domain is also a domain of holomorphy in the analysis of one complex variable, because of the Riemannian mapping theorem. ${ }^{7}$

\subsubsection{Multi-dimensional function theory}

The two statements just mentioned are incorrect for several complex variables $\mathbb{C}^{d}$, $d \geq 2$.

In several complex variables,

a domain is not necessarily a domain of holomorphy;

domains of holomorphy are usually not biholomorphically equivalent.

As compared to one-dimensional function theory, the emerging structures are thus very rich. Depending on the domain geometry, very different sheaves of holomorphic functions arise.

\section{Biholomorphic maps}

The notion of a biholomorphic map, which was used in statement (5.5), is the generalization of a conformal map to several complex variables. If a holomorphic mapping $m: U \rightarrow U^{\prime}$, with $U, U^{\prime} \subset \mathbb{C}^{d}$ is invertible, namely one-to-one, it is called a biholomorphic map. Two domains $U, U^{\prime}$ are biholomorphically equivalent, if and only if a biholomorphic map exists between them. They will have an equivalent sheaf of holomorphic functions. ${ }^{8}$ In contrast to conformal maps, biholomorphic maps do not necessarily preserve angles. Nevertheless, with respect to the holomorphic structure, they are the natural generalization of conformal maps on $\mathbb{C}$, because they are complex diffeomorphisms: the inverse of a biholomorphic map is also holomorphic [99]. Within the present thesis, it will be of practical use to exploit the biholomorphic equivalence of holomorphic functions on two-dimensional wedges.

\section{Domains of holomorphy and holomorphic envelopes}

Regarding statement (5.6), a remarkable example of a domain $\subset \mathbb{C}^{2}$ which is not a domain of holomorphy is given by the hollow sphere $M:=\left\{\underline{z}=\left(z_{1}, z_{2}\right)^{T} \in \mathbb{C}^{2}\right.$ : $\left.\frac{1}{2}<\sqrt{\left|z_{1}\right|^{2}+\left|z_{2}\right|^{2}}<1\right\}$. In sharp contrast to the single-variable case, one can show that any holomorphic function $f: M \rightarrow \mathbb{C}$ may be analytically continued

${ }^{7}$ For simplicity, let us just consider a simply connected bounded domain $G$. Due to the Riemann theorem it is conformally equivalent to the unit disk. The unit disk is a domain of holomorphy, because any simply connected set $\tilde{D}, \tilde{D} \supset D_{1}$, contains a number $\zeta$ on the unit circle. The function $1 /(z-\zeta)$ is holomorphic on $D_{1}$ but not on $\tilde{D}$. Thus, not all functions remain analytic on $\tilde{D}$. Due to conformal equivalence, $G$ is also a domain of holomorphy.

${ }^{8}$ Sheaves are very general mathematical concepts, but here one may just imagine the set of holomorphic functions $U \rightarrow \mathbb{C}$ carried by a given domain $U \subset \mathbb{C}^{d}$. 
to the unit sphere. The unit sphere is, in fact, a domain of holomorphy and is thus named the holomorphic envelope of $M$ [99]. This extends to the famous and surprising result by Friedrich Hartogs that isolated singularities are always removable for analytic functions $\mathbb{C}^{d} \rightarrow \mathbb{C}, d \geq 2$ (Hartogs' lemma). ${ }^{9}$ Isolated singularities play the fundamental role in the residue calculus of conventional complex analysis. This is a rather distinguished situation as compared to the $d \geq 2$ case, due to Hartogs' result. As will be shown in section 5.2, the theory of integral representations of complex functions for $d \geq 2$ has consequently a very different character as compared to the $d=1$ case.

We showed above that general domains such as the hollow sphere are often reducible to larger domains by means of the analytic continuation of its entire sheaf of holomorphic functions. For this reason, mathematicians restrict to the systematic study of corresponding envelopes of holomorphy, i.e. the domains of holomorphy. One can show that a domain is a domain of holomorphy if and only if it is a so-called pseudoconvex domain. Pseudoconvexity is a certain generalization of convexity from $\mathbb{R}^{d}$ to $\mathbb{C}^{d}[99]$. Whether or not a domain is reducible by means of its analytic structure is thus connected to its geometry. ${ }^{10}$

\section{Biholomorphic equivalence}

In order to provide a classification scheme for domains of holomorphy in $\mathbb{C}^{d}, d \geq 2$, the fruitful strategy from $d=1$, namely using biholomorphic equivalences, is adopted. Non-empty simply connected domains of holomorphy $\subsetneq \mathbb{C}^{d}$ are usually not biholomorphically equivalent. There are many different types of holomorphic structures, depending on the domain geometry. A prominent example of biholomorphically inequivalent domains of holomorphy is given by the unit ball $|\underline{z}|<1$ and the bicylinder $D_{1} \times D_{1}$ in $\mathbb{C}^{2}$. The unit ball in higher dimensions is with regard to the holomorphic structure in no way related to the unit disks and carries in fact a highly singular holomorphic structure. As mentioned above, in the present thesis, the equivalence class of two-dimensional wedges will become important. It is introduced in the following sections.

\subsection{Boundary value problems}

The Cauchy integral representation from conventional function theory can only be adopted for domains which are Cartesian products of bounded simply connected $\mathbb{C}$ domains, $G=G_{1} \times G_{2} \times \cdots \times G_{d}$. The function has to be continuous in $\bar{G}$ in

\footnotetext{
${ }^{9}$ Hartogs' lemma is also known as Osgood-Brown theorem. For further reference see the corollary in chapter III, section 17.4 of Ref. [99].

${ }^{10}$ In particular, for this reason, the notion of the holomorphic envelope is conceptually related to the notion of the convex envelope of a set. Note that the sphere is both, the holomorphic and the convex envelope of the hollow sphere.
} 
terms of the points in $\partial G_{1} \times \partial G_{2} \times \cdots \times \partial G_{d}{ }^{11}$ In some sense due to the surprising implications of Hartogs' lemma, finding a generalization of Cauchy integral representations to arbitrary domain geometries and function sets, yielding, for example, a spectral representation of our two-variable Green's function, turned out to be a cumbersome task. Many efforts were put in by mathematicians to find representations. At present, there are several, more or less general, possibilities to construct such representations, known, even with different dimensions of the integration manifolds. The Bochner-Martinelli representation ${ }^{12}$ is, for example, probably the most general integral representation, but the integration manifold is $2 d-1$ dimensional.

In our practical application, the integration space will become identical to a fit space in order to reconstruct real-time properties of the Green's function (cf. chapter 6 ). Of course, it is most desirable to keep the dimension of the fit space as small as possible. In the following, we aim at constructing a minimally redundant integral representation which will be tailored to the reconstruction of real-time properties.

\subsubsection{The Shilov Boundary}

The fact that there are different integral representations known which invoke different dimensionalities of function values or boundary values on the domain is clarified by the important concept of the distinguished boundary or Bergman-Shilov boundary ${ }^{13}$ of a bounded domain.

In $d=1$ complex analysis, Cauchy's integral representation reconstructs all values of a function on a simply connected open domain of finite radius using the limit values on the topological boundary, as long as the the boundary values are continuous. I.e. the structure on the topological boundary determines the structure of the interior. For $d>1$, the topological boundary can in this sense be reduced to an often much smaller set, the so-called distinguished or Bergman-Shilov boundary. In the 1930s, Stefan Bergman discovered the distinguished boundary in the context of $\mathbb{C}^{2}$ for bounded domains with piecewise smooth boundaries. He found that under certain regularity conditions, an integral representation with respect to the only twodimensional manifold $S$ of all intersections of the smooth boundaries was possible This invokes the so-called Bergman kernel function (cf. section 5.4.3). A precise geometric and analytic description of these results is provided in great detail by chapter XI of Ref. [106].

A pictorial comparison of the Bergman-Weil and the Cauchy representations is provided in figure 5.1. Bergman realized that the distinguished boundary $S$ is given

\footnotetext{
${ }^{11}$ See chapter IV in Ref. [99].

${ }^{12}$ For details see chapter IV, section 23 in Ref. [99].

13 The notion distinguished boundary was introduced in Bergman's book [106], in which analytic polyhedra (see below) in $\mathbb{C}^{2}$ were considered. For a more general discussion see the article on Bergman-Shilov boundaries in Ref. [107].
} 


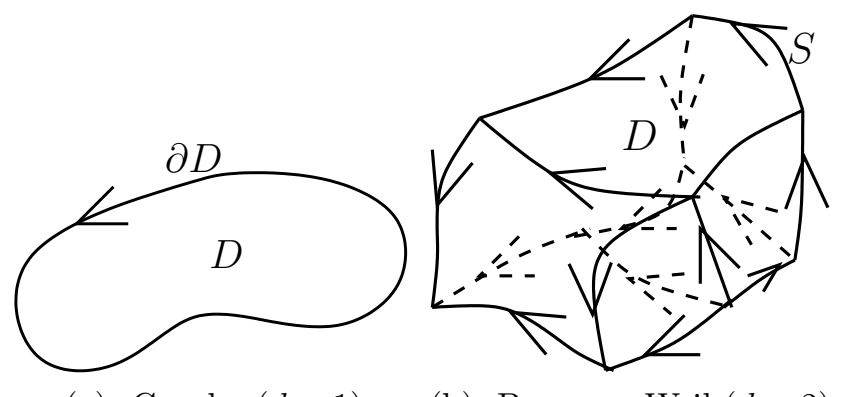

$\begin{array}{ll}\text { (a) Cauchy }(d=1) & \text { (b) Bergman-Weil }(d=2)\end{array}$

Figure 5.1: Comparison of Cauchy and Bergman-Weil integral representation theories. Integrations run over the (a) full topological boundary $\partial D$ and (b) the Bergman-Shilov boundary $S \subset \partial D$ of an analytic polyhedron, respectively.

by a maximum set $\tilde{S}$. This is a compact set $S \subset \bar{D}$ for which

$$
|f(\underline{z})| \leq \max _{\underline{\zeta} \in \tilde{S}}|f(\underline{\zeta})|
$$

for any $\underline{z} \in \bar{D}$, and any $f \in \mathcal{H}_{\bar{D}}$ where $\mathcal{H}_{\bar{D}}$ is the set of holomorphic functions $f: \bar{D} \rightarrow \mathbb{C}$. In the 1940 s, Shilov stumbled across the distinguished boundary in the rather different context of commutative Banach algebras. He showed that the intersection of all such maximum sets is nonempty and defines the distinguished boundary [107].

\section{Generalization}

Note that in modern terminology the Shilov boundary, as a generalization of the Bergman-Shilov boundary, may be defined for any compact space with respect to an algebra of continuous complex-valued functions on the space [107]. ${ }^{14}$ If we, for example, find physical constraints imposing certain conditions to the set of holomorphic functions, the Shilov boundary with respect to these functions may, in fact, "shrink" to a smaller subset of the Shilov boundary of the holomorphic functions. If a sufficiently elaborate kind of integral representation is used, this might enable us again to reduce the number of linear fit parameters significantly in the Bayesian inference problem.

\footnotetext{
${ }^{14}$ The considered algebra $\mathfrak{A}$ of continuous complex-valued functions also has to separate the points of the considered domain $G$, i.e. $\forall \underline{z}_{1}, \underline{z}_{2} \in G \exists f \in \mathfrak{A}: f\left(\underline{z}_{1}\right) \neq f\left(\underline{z}_{2}\right)$. This prevents to consider, e.g., the algebra of constant functions, for which $S$ would be empty.
} 


\section{Examples}

For the domains with piecewise smooth boundaries which Bergman investigated, $S$ is given by the unification of all possible intersections between the smooth boundary hypersurfaces, as long as certain regularity conditions hold. We return to our two examples from part 5.1.2, the bicylinder and the unit sphere and comment on their Bergman-Shilov boundaries.

The bicylinder, $D_{1} \times D_{1}$, is one of the most easily accessible domains of holomorphy, because it simply factorizes into two $D_{1}$-disks in $\mathbb{C}$. A minimal integral representation of a holomorphic function which is continuous on the closure $\overline{D_{1} \times D_{1}}$, is simply given by the product of two conventional Cauchy representations (see Theorem 2.2.1 in Ref. [108]),

$$
f(\underline{z})=\frac{1}{(2 \pi \mathrm{i})^{2}} \iint_{S_{1} \times S_{1}} \mathrm{~d}^{2} \zeta \frac{f(\underline{\zeta})}{\prod_{k}\left(z_{k}-\zeta_{k}\right)} .
$$

Therefore, the Bergman-Shilov boundary of $D_{1} \times D_{1}$ is given by the only twodimensional toroidal subset $S_{1} \times S_{1}=\partial D_{1} \times \partial D_{1}$ of the three-dimensional topological boundary $\partial\left(D_{1} \times D_{1}\right)=\partial D_{1} \times D_{1} \cup D_{1} \times \partial D_{1}$. Similarly, integral representations of domains which are direct products of $\mathbb{C}^{1}$ domains can be constructed from the conventional Cauchy integral formula.

It was already mentioned in part 5.1.2 that the unit sphere in $\mathbb{C}^{2}$ is not biholomorphically equivalent to the bicylinder. In contrast to the two connected smooth boundary hypersurfaces of the bicylinder, the boundary hypersurface of the unit sphere is not even a smooth hypersurface in Bergman's notion. One can show that here, the Bergman-Shilov boundary is, in fact, identical to the topological boundary. Thus, any integral representation for holomorphic functions in the unit sphere must invoke at least three real integrals. The unit sphere in $d \geq 2$ is thus a highly pathological type of domain. The intuition one might have from $d=1$ and from the fact that the bicylinder and the unit sphere are topologically equivalent is completely misguiding. The different dimensionalities of the distinguished boundary surfaces of the bicylinder and the unit sphere is an easy and intuitive indicator for the absence of a biholomorphic map between the two.

\subsection{Holomorphic Structure of the Green's Functions}

As a next step, the findings of section 3.4.2 shall be observed from the functiontheoretical point of view. The bare Green's function with respect to the two variables $\mathrm{i} \varphi_{m}, \mathrm{i} \omega_{n}$ reads $[27]$

$$
G_{0}\left(\mathrm{i} \varphi_{m}, \mathrm{i} \omega_{n}\right)=\sum_{\alpha= \pm 1} \frac{\Gamma_{\alpha} / \Gamma}{\mathrm{i} \omega_{n}-\alpha\left(\mathrm{i} \varphi_{m}-\Phi\right) / 2-\varepsilon_{d}+\mathrm{i} \Gamma \operatorname{sgn}_{n m}} .
$$


Here, $\operatorname{sgn}_{n m}:=\operatorname{sgn}\left(\omega_{n}-\alpha \varphi_{m} / 2\right)$. Performing the analytic continuations $i \omega_{n} \rightarrow z_{\omega}$, $\mathrm{i} \varphi_{m} \rightarrow z_{\varphi}$, the sgn function in the denominator results in an ambiguity, as far as the definition of domains for which $G_{0}$ is holomorphic is concerned.

Choosing a branch cut structure which corresponds to the continuation

$$
\operatorname{sgn}\left(\omega_{n} \pm \varphi_{m} / 2\right) \rightarrow \operatorname{sgn}\left(\operatorname{Im} z_{\omega} \pm \operatorname{Im} z_{\varphi} / 2\right)
$$

appears to be most sensible from both, a mathematic and a physical point of view:

First, in contrast to other choices the resulting domains are also domains of holomorphy and are thus "maximal" with respect to the holomorphic structure. ${ }^{15}$ The four domains of holomorphy are given by $\mathbb{C}^{2}$ separated into wedges by the two branchcut hyperplanes $\operatorname{Im} z_{\omega} \pm \operatorname{Im} z_{\varphi} / 2=0$. Second, it is just the imaginary part of the linear combinations of $z_{\omega}$ and $z_{\varphi}$, appearing in the denominators of perturbative expressions in $U$, which yields the crucial sign-switching delta functions generating the non-analytic terms separating the domains for which $G$ is holomorphic. See e.g. the structure of equation (3.81).

Consequently, for a finite many-body interaction $U \neq 0$, the introduction of a branch cut must be expected for any new kind of linear combination of $z_{\omega}$ and $z_{\varphi}$ in denominators of perturbative integrands. Second-order perturbation theory from Ref. [27] indicated that particle-hole bubbles create higher-order branch cuts $\operatorname{Im} z_{\omega}-\frac{\gamma}{2} \operatorname{Im} z_{\varphi}=0 ; \gamma$ odd, due to the structure of convolutions which are involved (see section 3.4.2). In a later publication [53] it was pointed out that vertex corrections appear to introduce yet another species of a branch cut, given by $\operatorname{Im} z_{\varphi}=0$. The physical retarded Green's function is then given by

$$
G_{\mathrm{ret}}(\omega)=\lim _{z_{\omega} \rightarrow \omega+\mathrm{i} 0^{+}} \frac{\lim _{\delta_{\varphi} \rightarrow 0^{+}}+\lim _{\delta_{\varphi} \rightarrow 0^{-}}}{2} G\left(\Phi+\mathrm{i} \delta_{\varphi}, z_{\omega}\right)
$$

i.e. one has to average over the two possible limits with respect to $\delta_{\varphi}$, in order to generate certain principal-value integrals of Keldysh perturbation theory. ${ }^{16}$

\subsubsection{Resulting Mathematical Assumptions}

The following assumptions are being made for the mathematical structure of the Green's function:

1. By means of holomorphy, we obtain cone-like constraints for the combinations of imaginary parts, as depicted in Fig. 3.4. More precisely, we require $G$ to be a solution of the Cauchy-Riemann equations (5.2) for any $\underline{z}^{(0)}$ except for those $\underline{z}^{(0)}$ which

$$
\operatorname{Im} z_{\omega}^{(0)}=\frac{\gamma}{2} \operatorname{Im} z_{\varphi}^{(0)} \vee \operatorname{Im} z_{\varphi}^{(0)}=0
$$

\footnotetext{
${ }^{15}$ They are "maximal" in the sense of a holomorphic envelope, cf. section 5.1.2.

${ }^{16}$ See the discussion in section 3.4.2.
} 
with some $\gamma \in 2 \mathbb{Z}+1$. Those $\underline{z}^{(0)}$ define the branch cut hyperplanes and delimit the wedges for which $G$ is holomorphic.

2. We will require the interacting Green's function $G(\underline{z})$ to be bounded, i.e.

$$
\sup _{\underline{z} \in \mathbb{C}^{2}}|G(\underline{z})|<\infty .
$$

3. We will assume that the Green's function $G(\underline{z})$ is uniquely defined by the discrete function values $G\left(\mathrm{i} \varphi_{m}, \mathrm{i} \omega_{n}\right)$ which are obtained from the effectiveequilibrium computations. I.e. we require that the continuation to a multisheeted holomorphic function

$$
G\left(\mathrm{i} \varphi_{m}, \mathrm{i} \omega_{n}\right) \rightarrow G\left(z_{\varphi}, z_{\omega}\right)
$$

is unique.

The second assumption is justified by the structure of the convolution equations (Wick contractions) in perturbation theory and the boundary conditions that terms $\mathrm{e}^{\mathrm{i} \varphi_{m} \beta / 2}$ and $\mathrm{e}^{\mathrm{i} \omega_{n} \beta}$, evaluate to 1 and -1 before the analytic continuations are carried out.

A proof of the third assumption, which is of course crucial for the physical theory itself, is provided in appendix A. It is based on assumption 1 and 2 and the assumption 3' which sharpens the requirements on the $\underline{z} \rightarrow \infty$-asymptotics:

3'. Given arbitrary $\underline{x}^{(0)} \in \mathbb{R}^{2} \backslash\{0\}$ and $\zeta \in \mathbb{C}$, we have

$$
\lim _{\zeta \rightarrow \infty}\left|\zeta \cdot G\left(\zeta \underline{x}^{(0)}\right)\right|<\infty \Leftrightarrow x_{\omega}^{(0)} \neq \pm x_{\varphi}^{(0)} / 2 .
$$

In other words, $G(\underline{z})$ is required to decay like a usual Green's function as a function of $\zeta$, where $\underline{z}=\zeta \cdot \underline{x}^{(0)}$, if and only if the direction $\underline{x}^{(0)}$ satisfies the regularity condition $x_{\omega}^{(0)} \neq \pm \bar{x}_{\varphi}^{(0)} / 2$.

\subsubsection{Justification of assumption 3'}

The assumption 3' may be justified as follows.

Consider the absolute value of the free Green's function (5.9), $\left|G_{0}\left(z_{\varphi}, z_{\omega}\right)\right| \leq$ $\sum_{\alpha= \pm 1} \frac{\Gamma_{\alpha}}{\Gamma} \frac{1}{\mid\left(z_{\omega}-\alpha\left(z_{\varphi}-\Phi\right) / 2-\varepsilon_{d}+\mathrm{i} \Gamma \operatorname{sgn} I m\left(z_{\omega}-\alpha z_{\varphi} / 2\right) \mid\right.}$. It is obvious that it decays $\propto \frac{1}{\zeta}$ when $z_{\varphi}=\zeta \cdot x_{\varphi}^{(0)}$ and $z_{\omega}=\zeta \cdot x_{\omega}^{(0)}$ for the nonsingular combinations of $x_{\varphi}^{(0)}$ and $x_{\omega}^{(0)}$. It does not decay at all in the singular cases $x_{\omega}^{(0)}= \pm x_{\varphi}^{(0)} / 2$. 
It is easy to check that interaction $U>0$ does not alter this high-energy structure. Let us examine the second-order selfenergy expression (Eq. (15) in Ref. [27]):

$$
\Sigma^{(2)}(\underline{z})=U^{2} \sum_{\alpha_{i}}\left[\prod_{i=1}^{3} \int \mathrm{d} \varepsilon_{i} \frac{\Gamma_{\alpha_{i}}}{\Gamma}\right] \cdot \frac{f_{\alpha_{1}}\left(1-f_{\alpha_{2}}\right) f_{\alpha_{3}}+\left(1-f_{\alpha_{1}}\right) f_{\alpha_{2}}\left(1-f_{\alpha_{3}}\right)}{z_{\omega}-\left(\alpha_{1}-\alpha_{2}+\alpha_{3}\right) \frac{z_{\varphi}-\Phi}{2}-\varepsilon_{1}+\varepsilon_{2}-\varepsilon_{3}},
$$

with $f_{\alpha_{i}}=f\left(\varepsilon_{i}-\alpha_{i} \Phi / 2\right)$.

Due to the structure of the denominator, we see that on top of the singular directions of the bare Green's function, $x_{\omega}^{(0)}= \pm x_{\varphi}^{(0)} / 2$, we also have the singular directions $x_{\omega}^{(0)}= \pm \frac{3}{2} x_{\varphi}^{(0)}$.

Consequently, assumption 3' is incorrect for the second-order selfenergy. Nevertheless, when inserted into Dyson's equation,

$$
G^{(2)}(\underline{z})=\frac{G_{0}(\underline{z})}{1-G_{0}(\underline{z}) \Sigma^{(2)}(\underline{z})}
$$

we see that for the directions $x_{\omega}^{(0)}= \pm \frac{3}{2} x_{\varphi}^{(0)}$ the limiting behaviour of $G_{0}$ is adopted, i.e. the behaviour (5.15). Note that the uniqueness proof of appendix A also holds when applied directly to the selfenergy, because the singular directions of the secondorder perturbation theory, $x_{\omega}^{(0)}= \pm \frac{3}{2} x_{\varphi}^{(0)}$, are not required to be regular in the proof. This is because the direction $x_{\omega}^{(0)}= \pm \frac{3}{2} x_{\varphi}^{(0)}$ also defines a branch cut (assumption 1).

\subsection{Tubular Cone Domains (“Wedges”)}

As we have seen, the structure of $G_{0}$ combined with the structure of convolutions in the perturbation theory with respect to $U$, indicate that the numerous branch cut hyperplanes divide $\mathbb{C}^{2}$ into several, in fact infinitely many, wedges of the form $T^{C}=\mathbb{R}^{2}+\mathrm{i} C$. $C$ is a convex cone with vertex at zero. See also figure 3.4. Due to the convexity of $C, T^{C}$ is pseudoconvex and is thus a domain of holomorphy, i.e. in some sense irreducible with respect to its analytic structure [99]. ${ }^{17}$ In the function-theoretical classification scheme, domains like these are called tubular cone domains.

\subsubsection{Geometry of the Cones}

We briefly introduce certain notions of the description of the analytic geometry of cones in $\mathbb{R}^{d}$. This is necessary to thoroughly follow the mathematical formulae which are involved in the description of the analytic structure of $T^{C}$. A cone $C$ with vertex at zero is formally defined by the scaling property $\underline{x} \in C \Rightarrow \forall \lambda>0: \lambda \underline{x} \in C$.

\footnotetext{
17 See the discussion of holomorphic envelopes in section 5.1.2.
} 
Its dual cone $C^{*}$ is defined via the standard scalar product

$$
(\underline{\xi}, \underline{x}):=\sum_{k=1}^{d} \xi_{k} x_{k} ; \quad \underline{\xi}, \underline{x} \in \mathbb{R}^{d},
$$

by

$$
C^{*}:=\left\{\underline{\xi} \in \mathbb{R}^{d} \mid \forall \underline{x} \in C:(\underline{\xi}, \underline{x}) \geq 0\right\} .
$$

$C^{*}$ represents the space of positive semi-definite linear functionals on $C$ when the functional form (5.18) is considered. The dual cone is important, because the construction of kernel functions often involves Fourier transforms.

A convex cone is a cone for which the straight line between any pair of points within the cone is also contained in the cone. We will also use the analytic continuation of the scalar product (5.18) with respect to $\underline{x}$. We continue $\underline{x} \rightarrow \underline{z}$ holomorphically in (5.18):

$$
(\underline{\xi}, \underline{z}):=\sum_{k=1}^{d} \xi_{k} z_{k} ; \quad \underline{\xi} \in \mathbb{R}^{d}, \underline{z} \in \mathbb{C}^{d} .
$$

\subsubsection{Analytic Structure and Biholomorphic Equivalence Classes}

Tubular cones and domains are very well-known objects in the theory of several complex variables, because they naturally arise in certain fields of mathematics. As a consequence, many efforts were put in a detailed understanding of their structure. In the prominent physical example, axiomatic quantum field theory, the cones represent forward and backward light cones, in four-dimensional spacetime, $d=4$. A celebrated result was Bogolyubov's edge-of-the-wedge theorem. ${ }^{18}$

A key component of the wedge is given by its edge, namely the real subspace associated with the vertex of the cone. Because in our case the vertex is located at zero, the edge of the wedge $T^{C}$ can be formally identified with an oriented copy of the real subspace,

$$
\operatorname{Edge}_{T^{C}}:=\mathbb{R}^{d}+\mathrm{i}^{C},
$$

where $0^{C}$ is an infinitesimal vector within the cone $C$. Although there exist, depending on the direction approaching the origin within $C$, several infinitesimals $0^{C}$, the Edge is well-defined through (5.21), because all infinitesimals in $C$ are obviously equivalent with respect to holomorphic continuation in $T^{C} . T^{C}$ may be regarded as a generalization of the upper half plane in $\mathbb{C}$.

Let $C, C^{\prime}$ be arbitrary convex cones in $\mathbb{R}^{d}, C \neq C^{\prime} . T^{C}$ and $T^{C^{\prime}}$ are in general not biholomorphically equivalent. However, our two-dimensional case is fortunately an exception to this rule: $T^{C}$ and $T^{C^{\prime}}$ are biholomorphically equivalent for any combination $C, C^{\prime}$. See also the introductory notes in the corresponding part of the second

\footnotetext{
${ }^{18}$ The edge-of-the-wedge theorem will be briefly discussed in section 7.1 .
} 
volume of Ref. [34]. In $\mathbb{C}^{2}$, biholomorphisms between $T^{C}$ and $T^{C^{\prime}}$ may be constructed easily using complexified rotations and dilations of $\mathbb{R}^{2}$ : Consider that the real (nonsingular) matrix $M: \mathbb{R}^{2} \rightarrow \mathbb{R}^{2}, \underline{x} \mapsto\left(\begin{array}{ll}a & b \\ c & d\end{array}\right) \underline{x}$ maps $C$ to $C^{\prime}$, i.e. $C^{\prime}=M C$. The corresponding biholomorphism between $T^{C}$ and $T^{C^{\prime}}$ is obtained from the complexified map $\tilde{M}: \mathbb{C}^{2} \rightarrow \mathbb{C}^{2}, \underline{z} \mapsto\left(\begin{array}{ll}a & b \\ c & d\end{array}\right) \underline{z}$. It is easy to see that $T^{C^{\prime}}=M T^{C}$, that $M$ is holomorphic, and invertible. If the complexified linear map is a rotation, we will also call it a biholomorphic rotation when we want to emphasize the biholomorphic character of the mapping.

Note that the helpful notion of the Bergman-Shilov boundary is not directly applicable to $T^{C}$, because $T^{C}$ is unbounded. However, as will be discussed next, sequences of bounded domains approaching $T^{C}$ from its interior may be used to understand the holomorphic structure on $T^{C}$.

\subsubsection{Bergman-Weil Representations}

The following three subsections describe how not to build an integral representation for wedges of the two-variable Green's function of Matsubara voltage theory. By this, some insightful peculiarities of the theory analytic structure of the theory are revealed. The proper integral representation is then derived in section 5.5, based on the Cauchy-Bochner integral kernel which is introduced in section 5.4.4.

We may construct a sequence of bounded domains $\mathcal{D}_{n} \subset T^{C}, \lim _{n \rightarrow \infty} \mathcal{D}_{n}=T^{C}$, with piecewise smooth boundaries like in Fig. $5.1 \mathrm{~b}$ easily, such that the edge of the wedge contains a part of the Bergman-Shilov boundary $\mathcal{S}_{n}$ of $\mathcal{D}_{n}$ and such that the other subsets of $\mathcal{S}_{n}$ disappear to $\infty$ as $n \rightarrow \infty$. In our case, $d=2$, an explicit construction of such a sequence may be obtained by intersecting $T^{C}$ with a growing bicylinder $\mathcal{B}_{n}:=R_{n} \cdot\left(D_{1} \times D_{1}\right), \mathcal{D}_{n}:=\mathcal{B}_{n} \cap T^{C}$, with $R_{n} \propto n$. The procedure is sketched in Fig. 5.2. One finds that the sequence of Bergman-Shilov boundaries improperly converges to

$$
\mathcal{S}_{\infty}:=\operatorname{Edge}_{T^{C}} \cup\{\infty\},
$$

where " $\infty$ " shall informally denote the point or merely a list of points which emerge when $T^{C}$ is, depending on the holomorphic structure, compactified suitably. ${ }^{19}$ At first glance, each direction for approaching $\infty$ might yield a different point in $\{\infty\}$. The points " $\infty$ " carry the additional information which is necessary to turn the structure on Edge ${ }_{T_{C}}$ into a unique description of the holomorphic structure on $T^{C}$.

\footnotetext{
${ }^{19}$ This informal terminology shall just emphasize the fact that the sequence of Shilov boundaries leads to a non-trivial structure " $\infty$ " at infinity which needs to be taken care of when an integral representation is constructed. Fortunately, however, the finite part of $\mathcal{S}_{\infty}$, namely the edge of $T^{C}$, is just the real-time limit of the holomorphic branch of the Green's function on $T^{C}$ enclosed by two subsequent branch cuts (cf. figure 3.4).
} 


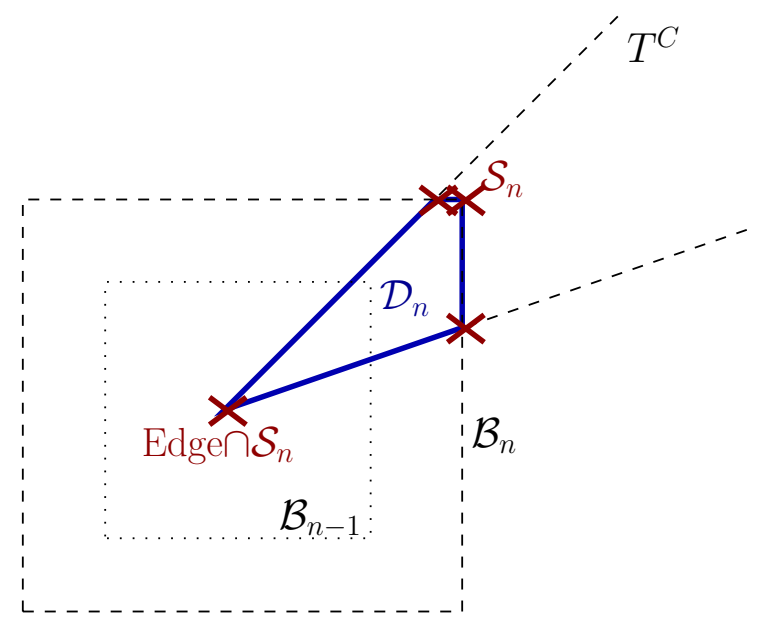

Figure 5.2: An artist's impression of asymptotically filling the wedge $T^{C}$ with the sequence $\mathcal{D}_{n} . \quad \mathcal{D}_{n}$ is a domain with piecewise smooth boundaries created by intersecting with a growing bicylinder $\mathcal{B}_{n}$, creating the analytic polyhedra $\mathcal{D}_{n}$. The corresponding sequence of Bergman-Shilov boundaries improperly converges to the set Edge $\cup\{\infty\}$. Therefore, the sheaf of holomorphic functions on $T^{C}$ is solely characterized by its edge values and its asymptotic behaviour $\underline{z} \rightarrow \infty$.

The Bergman representation for an analytic polyhedron in $\mathbb{C}^{2}$ may be obtained by the following procedure [106]. An analytic polyhedron is defined by

$$
\zeta^{(k)}=\mathfrak{f}^{(k)}\left(\underline{z}, \lambda_{k}\right)
$$

where $\mathfrak{f}^{(k)}\left(\underline{z}, \lambda_{k}\right)$ are $\lambda$-parametrized families of analytic functions of $\underline{z}$ and $\lambda_{k} \in$ $\Lambda_{k} \subset \mathbb{R}, k=1, \ldots, K$. Each equation yields a surface in $\underline{z}$-space for a given $\lambda$ and a hypersurface $h_{k}$ in $\underline{z}$-space as $\lambda$ is varied continuously. The mutual intersections $S_{k l}=h_{k} \cap h_{l}$ yield the Bergman-Shilov boundary surface $S=\bigcup_{k, l} S_{k l}$. $S_{k l}$ is then parametrized by a function $\underline{z}=\underline{\mathfrak{g}}^{(k l)}\left(\lambda_{k}, \lambda_{l}\right)$. A holomorphic function $f$ on the analytic polyhedron may then be written with respect to the Bergman-Shilov boundary of the latter using the Bergman kernel function [106]

$$
B_{k l}=\left|\frac{\partial\left(\mathfrak{g}_{1}^{(k l)}, \mathfrak{g}_{2}^{(k l)}\right)}{\partial\left(\lambda_{k}, \lambda_{l}\right)}\right| \cdot\left(\frac{\mathfrak{f}_{l}\left(z_{1}, z_{2}, \lambda_{l}\right) \mathfrak{f}_{k}\left(z_{1}, \mathfrak{g}_{2}^{(k l)}, \lambda_{k}\right)}{\left(\mathfrak{g}_{1}^{(k l)}-z_{1}\right)\left(\mathfrak{g}_{2}^{(k l)}-z_{2}\right)}-\frac{\mathfrak{f}_{k}\left(z_{1}, z_{2}, \lambda_{k}\right) \mathfrak{f}_{l}\left(z_{1}, \mathfrak{g}_{2}^{(k l)}, \lambda_{l}\right)}{\left(\mathfrak{g}_{1}^{(k l)}-z_{1}\right)\left(\mathfrak{g}_{2}^{(k l)}-z_{2}\right)}\right) .
$$

The integral representation with respect to $\left.f\right|_{S}$ then reads

$$
f(\underline{z})=-\frac{1}{8 \pi^{2}} \sum_{k \neq l} \int_{\lambda_{k}, \lambda_{l}} \frac{f\left(\mathfrak{g}_{1}^{(k l)}, \mathfrak{g}_{2}^{(k l)}\right) B_{k l}}{\mathfrak{f}^{(k)}\left(\underline{z}, \lambda_{k}\right) \mathfrak{f}^{(l)}\left(\underline{z}, \lambda_{l}\right)}
$$


and can be applied directly to our $\mathcal{D}_{n}$ domains.

An explicit test on whether a Bergman integral representation for $G_{0}$ on the $\mathcal{D}_{n}=$ $\mathcal{B}_{n} \cap T^{C}$ domains is feasible yields that the subsets of $\mathcal{S}_{n}$ which go to $\infty$ may not be neglected for $G(\underline{z})$ functions. ${ }^{20}$ This is because $G_{0}(\underline{z})$ has a nonzero limit as $\underline{z} \rightarrow \infty$ if one goes along the cross-shaped submanifold $\operatorname{Re} z_{\omega}= \pm \operatorname{Re} z_{\varphi} / 2$ and keeps $\operatorname{Im} \underline{z}$ constant. Due to the independence from Im $\underline{z}$, this problem occurs for each of the wedges. Hence, the Bergman kernel function (5.24) is only of limited practical use for the two-variable Green's functions.

However, the formal use of the sequence $\mathcal{D}_{n}$ enables us to see very explicitly that the Edge of $T^{C}$ is the only carrier of structural information which involves finite values of $\underline{z}$. The rest of the information, then uniquely defining the holomorphic structure on $T^{C}$, is encoded in the several possible classes of limiting behaviour as $\underline{z}$ approaches infinity.

\subsubsection{Cauchy-Bochner Integral Representation}

As an almost straightforward consequence of this, assuming a certain limiting behaviour of the considered set of functions on $T^{C}$, integral representations with respect to the Edge $\mathbb{R}^{d}+\mathrm{i}^{C}$ may be derived. Even more generally, a constraint on the function class which also limits the Edge behaviour, can be imposed in such a way that the Edge function yields a unique description. The several possible $\underline{z} \rightarrow \infty$ behaviours are then, using the information from the constraint, encoded in the Edge. This appears to be linked deeply to the extension of the notion of the Bergman-Shilov boundary to the notion of the Shilov boundary mentioned in the course of section 5.2.1: Considering only a subset of the sheaf of holomorphic functions on a certain (compactified) domain may cause the Shilov boundary to "shrink".

One of the earliest of such developments was another extension of the Cauchy integral formula with respect to tubular cones by Salomon Bochner. ${ }^{21}$ It goes beyond restricting the considered function set such that a naïve extrapolation of Bergman representations, like in Fig. 5.2, holds. It considers a function $f$ which is holomorphic on $T^{C}$ and satisfies the constraint

$$
\|f(\underline{x}+\mathrm{i} \underline{y})\| \leq M_{\varepsilon, f}\left(C^{\prime}\right) \mathrm{e}^{\varepsilon|y|}
$$

which has to hold for any compact ${ }^{22}$ cone $C^{\prime} \subset C$ and for any $\varepsilon>0$, where $M_{\varepsilon, f}\left(C^{\prime}\right)$ is a suitably chosen real number. $\|\cdot\|$ is a norm which integrates out the $\underline{x}$ variable,

20 We aim at a parametrization of the function with respect to the real-time structure which corresponds to the edge of $T^{C}$. Therefore, getting rid of the explicit dependence on the structure at " $\infty$ " is desirable. However, it cannot be accomplished by simply "extrapolating" Bergman-Weil representations.

${ }^{21}$ See chapter IV, section 25 in Ref. [99].

${ }^{22}$ See paragraph 1 in section 25 of chapter IV of Ref. [99] for an appropriate definition of the compact subcone. 
$\|f(\underline{x}+\mathrm{i} \underline{y})\|^{2}:=\int \mathrm{d}^{d} x|f(\underline{x}+\mathrm{i} \underline{y})|^{2}[99]$.

Inequality (5.26) constrains the limiting behaviour $\underline{z} \rightarrow \infty$ in a sufficiently strong way such that an integral representation with respect to the Edge may be constructed. $^{23}$ Namely, the Cauchy-Bochner representation then allows a function $f$ satisfying (5.26) to be written as

$$
f(\underline{z})=\frac{1}{(2 \pi)^{d}} \int \mathrm{d}^{d} x^{\prime} \mathcal{K}_{C}\left(\underline{z}-\underline{x}^{\prime}\right) f\left(\underline{x}^{\prime}+\mathrm{i} 0^{C}\right) ;
$$

with the Edge values $f\left(\underline{x}^{\prime}+\mathrm{i}^{C}\right)$. Here, the so-called Cauchy kernel [99] of the cone $C$,

$$
\mathcal{K}_{C}(\underline{z}):=\int_{C^{*}} \mathrm{~d}^{d} \xi \mathrm{e}^{\mathrm{i}(\underline{\xi}, \underline{z})},
$$

was introduced. It is straightforward to compute the Cauchy kernel for our wedges with this formula. We will provide a general but easily applicable expression for further numerical and analytical computations in section 5.6.

Unfortunately, as in the Bergman approach, a numerical test of Eq. (5.27) for $f=G_{0}$ using an arbitrary wedge for which $G_{0}$ is holomorphic shows that the CauchyBochner representation (5.27) is also incorrect for $G_{0}$. As a consequence, we find that the Green's functions does not satisfy (5.26). This is compatible with the fact that the left hand side of (5.26) diverges in the case $f=G_{0}$, no matter which $C^{\prime}$ is considered.

Nevertheless, as we will see, the Cauchy-Bochner kernel will serve as a building block for the construction of a kernel for a different class of holomorphic functions which in fact contains our Green's functions $G(\underline{z})$ when a single wedge is considered.

\subsubsection{The tubular octant $\mathbb{H} \times \mathbb{H}$ and Biholomorphic Equivalence to the Bicylinder}

Before coming to the practically usable integral equation, one further conceptually important mapping should be discussed, which is in fact essential for the applicability of the actual representation derived in section 5.5. Due to the biholomorphic equivalence of all $T^{C}$ in $\mathbb{C}^{2}$, any of the wedges which arise for the Green's function may be mapped biholomorphically to the tubular octant $\mathbb{H} \times \mathbb{H}=\mathbb{R}^{2}+\mathrm{i}\left(\mathbb{R}^{+} \times \mathbb{R}^{+}\right)$, where $\mathbb{H}$ is the upper half plane of $\mathbb{C}$. This domain may itself be mapped biholomorphically to the bicylinder $D_{1} \times D_{1}$ via a piecewise Möbius transformation of the coordinates. Hence, all wedges of the Green's function are biholomorphically equivalent to the bicylinder. Let us denote a corresponding biholomorphism by $\mathfrak{m}_{C}: T^{C} \rightarrow D_{1} \times D_{1}$. We would like to comment on this due to the striking simplicity of the bicylinder and of

\footnotetext{
${ }^{23}$ This is possibly because the Shilov boundary of the constrained set of holomorphic functions shrinked to the edge. See the discussion of the generalized notion in section 5.2.1.
} 


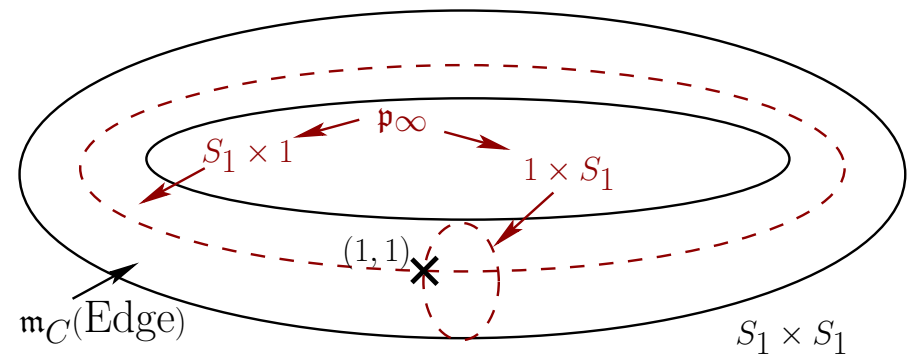

Figure 5.3: On the distinguished boundary surface of the bicylinder, the picture of $\operatorname{Edge}_{T^{C}}$ under the component-wise Möbius transformation $\mathfrak{m}_{C}$ is delimited by the dash-dotted lines $\mathfrak{p}_{\infty}$. A discontinuity of $\left.G\right|_{T^{C}} \circ \mathfrak{m}_{C}^{-1}$ occurs at the intersection point $(1,1)$ of the two circles and prevents a Cauchy representation from being applicable. The violation of the CauchyBochner condition (5.26) appears to be related to the occurance of the discontinuity.

domains which are direct products of $\mathbb{C}^{1}$ domains with respect to the construction of integral representations. From the point of view of this construction, the tubular octant may be regarded as the simplest representant of the biholomorphic equivalence class of all wedges in $\mathbb{C}^{2}$.

Due to the premises of the Cauchy integral formula, a usage of the representation (5.8) for the biholomorphically transformed sheaf of holomorphic functions is feasible in case the transformed Green's function $\left.G\right|_{T^{C}} \circ \mathfrak{m}_{C}^{-1}$ is continuous on its topological boundary $\partial\left(D_{1} \times D_{1}\right) .{ }^{24}$ Note that under the biholomorphic transformations, $S_{1} \times S_{1}$ is mapped to the edges of the $T^{C}$ wedges. The " $\infty$ " in $T^{C}$ maps to " $\infty$ " in $\mathbb{H} \times \mathbb{H}$ under biholomorphic transformation, and that again maps biholomorphically, using the component-wise Möbius transformation $\frac{z_{k}-\mathrm{i}}{z_{k}+\mathrm{i}}$ to the points $\mathfrak{p}_{\infty}:=\{1\} \times S_{1} \cup S_{1} \times\{1\}$. Note that the points $\mathfrak{p}_{\infty} \subset \partial\left(D_{1} \times D_{1}\right)$ are also part of the distinguished boundary torus $S_{1} \times S_{1}$.

The boundary behaviour of the transformed Green's function $\left.G\right|_{T^{C}} \circ \mathfrak{m}_{C}^{-1}$ is not continuous at the intersection point $(1,1)$ of the two circles $\mathfrak{p}_{\infty}$, due to the properties of $\left.G_{0}\right|_{T^{C}}$ at $\infty$ leading to singular directions, as summarized in assumption 3' of section 5.3.

An illustration may be found in Fig. 5.3.

Therefore, using the biholomorphic equivalence to the bicylinder is not immediately helpful for the construction of an integral representation of the Green's function $G$. Nevertheless, it is essential in the application of Vladimirov's approach which will be subject of the next section.

\footnotetext{
$\overline{{ }^{24} \text { More precisely, it must be continuous }}$ in the closure $\overline{D_{1} \times D_{1}}$ at all points on the distinguished boundary torus $S_{1} \times S_{1}$ shown in figure 5.3. See chapter IV in Ref. [99].
} 


\subsection{Vladimirov's Integral Formula}

In the late 1970s and early 1980s, Vladimirov provided a generalization [34; 109] of the so-called Herglotz-Nevanlinna representation to tubular cone domains which is, due to its generality, applicable to the interacting Green's function $G$. His investigations were motivated by applications in the field of linear passive systems in mathematical physics.

\subsubsection{Herglotz-Nevanlinna Representation $(d=1)$}

Herglotz' representation theorem considers holomorphic functions in the open unit disk $D_{1}$ which have a positive real part, the so-called Carathéodory functions [110112]. By separating a phase factor out of the function, one can also consider functions with positive or negative imaginary part, and so on. Since the open unit disk can be conformally mapped to the upper half plane $\mathbb{H}$, using the Möbius transformation $\frac{z-\mathrm{i}}{z+\mathrm{i}}$, as mentioned above, the representation can, under certain circumstances, be also used for $\mathbb{H}$.

By considering Carathéodory functions, Herglotz' theorem only imposes assumptions on the positivity of the real (imaginary) part of the function. In contrast to Cauchy's integral formula, no assumptions about the behaviour of the Carathéodory functions on the boundary of the disk are made, such as the continuity. The theorem states that every Carathéodory function $f$ can be represented by

$$
f(z)=\mathrm{i} \cdot \operatorname{Im} f(0)+\int_{0}^{2 \pi} \frac{\mathrm{e}^{\mathrm{i} t}+z}{\mathrm{e}^{\mathrm{i} t}-z} \mathrm{~d} \sigma(t)
$$

where $\mathrm{d} \sigma$ is a nonnegative finite measure [110].

Regarding a different set of functions, a formally very similar representation is the so-called Poisson formula, ${ }^{25}$ which is the analog of the Cauchy formula to the real analysis of harmonic functions, and can in fact be derived from it. It provides an integral kernel for the solution to the Dirichlet problem for the Laplace equation on the unit disk in $\mathbb{R}^{2}$. For a continuous function $\mathfrak{f}: \partial D_{1} \rightarrow \mathbb{R}$ it allows to construct a harmonic function $u: D_{1} \rightarrow \mathbb{R}$ as follows:

$$
u(z)=\frac{1}{2 \pi} \operatorname{Re} \int_{0}^{2 \pi} \frac{\mathrm{e}^{\mathrm{i} t}+z}{\mathrm{e}^{\mathrm{i} t}-z} \mathfrak{f}\left(\mathrm{e}^{\mathrm{i} t}\right) \mathrm{d} t
$$

A comparison of Eqs. (5.29) and (5.30) shows that the measure $\mathrm{d} \sigma$ of the Herglotz formula is in fact defined by the (possibly singular) boundary limit of the holomorphic function.

\footnotetext{
${ }^{25}$ See pp. 169ff. in Ref. [113].
} 
As a natural extension of the Poisson formula, the Schwarz integral formula ${ }^{26}$ reconstructs a holomorphic function $f$ on the closed unit disk from the real part of its boundary values, up to a constant imaginary offset. It reads

$$
f(z)=\mathrm{i} \cdot \operatorname{Im} f(0)+\frac{1}{2 \pi} \int_{0}^{2 \pi} \frac{\mathrm{e}^{\mathrm{i} t}+z}{\mathrm{e}^{\mathrm{i} t}-z} f\left(\mathrm{e}^{\mathrm{i} t}\right) \mathrm{d} t .
$$

Apparently, the only formal difference between (5.30) and (5.31) is the different measure.

Due to the conformal equivalence, for a holomorphic function $f$ on the closed upper half plane $\operatorname{Im} z \geq 0$, under the assumption that there is an $\alpha>0$ for which $\left|z^{\alpha} f(z)\right|$ is bounded, one has the Schwarz representation in the following form:

$$
f(z)=\frac{1}{\pi \mathrm{i}} \int_{-\infty}^{\infty} \frac{\operatorname{Re} f\left(x+\mathrm{i} 0^{+}\right)}{x-z} \mathrm{~d} x .
$$

Note the formal equivalence to the spectral representation of a conventional Matsubara Green's function [97], $G(z)=\int \mathrm{d} x \frac{1}{-\pi} \frac{\operatorname{Im} G\left(x+\mathrm{i} 0^{+}\right)}{z-x}$.

Similarly, the Poisson kernel for the closed upper half plane is

$$
P_{y}(x)=\frac{y}{x^{2}+y^{2}}
$$

yielding the representation

$$
u(x+\mathrm{i} y)=\frac{1}{\pi} \int_{-\infty}^{\infty} P_{y}(x-t) \mathfrak{f}(t) \mathrm{d} t,
$$

with $\mathfrak{f} \in L^{p}(\mathbb{R})$.

The full Herglotz-Nevanlinna representation [109] of arbitrary analytic functions with positive real part for the open upper half-plane reads

$$
\begin{aligned}
f(z)= & \frac{z+\mathrm{i}}{\pi \mathrm{i}} \int \frac{\mathrm{d} \mu\left(x^{\prime}\right)}{\left(x^{\prime}-\mathrm{i}\right)\left(x^{\prime}-z\right)} \\
& -\frac{1}{\pi} \int \frac{\mathrm{d} \mu\left(x^{\prime}\right)}{1+x^{\prime 2}}-\mathrm{i} a z+b .
\end{aligned}
$$

Here, $\mu$ is given by the boundary-value distribution of $\operatorname{Re} f$ :

$$
\mu=\operatorname{Rebv} f
$$

${ }^{26}$ See p. 171 in Ref. [113]. 
i.e. $\mu(x)^{"}=" \operatorname{Re} f\left(x+\mathrm{i} 0^{+}\right)$. The linear coefficient

$$
a=\operatorname{Re} f(\mathrm{i})-\frac{1}{\pi} \int \frac{\mathrm{d} \mu\left(x^{\prime}\right)}{1+x^{\prime 2}},
$$

and the constant term $b=\operatorname{Im} f(\mathrm{i})$. For example, in the case of the function $f(z)=$ $\mathrm{i} / \pi z, \mu$ is the Dirac measure $\mu(x)=\operatorname{Rebv} f=\delta(x)$ and the coefficients $a=b=0$. The case $\mu(x)=-a \cdot \delta(x)$ is not permitted by construction.

At first glance, with regard to their formal structure Eqs. (5.32) and (5.34) and the connection to the Herglotz-Nevanlinna representation (5.35) seem to be rather straightforward applications of the Cauchy integral formula. However, attempting the multidimensional generalization, we found that in our case, $d>1$, the CauchyBochner way of invoking $\mathcal{K}_{C}$ for a representation, equation (5.28), is not valid for the noninteracting Green's function $\left.G_{0}\right|_{T^{C}}$ : see section 5.4.4. Remarkably, as pointed out in section 5.4.5, taking assumption 2 from section 5.3 into account, we find that when transformed to the bicylinder, the Green's function $\left.G\right|_{T^{C}} \circ \mathfrak{m}_{C}^{-1}$ is very similar to a Carathéodory function, but the Cauchy-Bochner representation is invalid. These subtleties are apparently reflected by the central assumption (5.26) of CauchyBochner representations.

\subsubsection{Functions with positive real or imaginary part in $T^{C}$}

Note that the representation (5.35) can be also used for bounded functions on the upper half plane. This can be formally accomplished by introducing a shift in $f$ which makes the real part of the function of consideration positive definite. While the signs of its real and imaginary parts will vary, the considered holomorphic sheet of the Green's function, $\left.{ }^{27} G\right|_{T^{C}}$, is guaranteed to be bounded, due to assumption (5.13).

It is thus worthwhile to study the set of holomorphic functions with positive imaginary parts on $T^{C}$ which shall be denoted by $H_{+}\left(T^{C}\right)$. Due to the biholomorphic equivalence of the Green's function's wedges to the bicylinder, one may think of $H_{+}\left(T^{C}\right)$ as a generalization of the Carathéodory functions. Note that in the literature, sometimes functions with positive real and sometimes functions with positive imaginary parts are considered, resulting in marginal differences in the equations.

\footnotetext{
$\overline{27}$ Please recall that holomorphic sheets of the Green's function are enclosed by subsequent branch cuts, as depicted in figure 3.4.
} 


\subsubsection{Vladimirov's Kernel Functions for $T^{C}$}

We will now study the generalization of the Herglotz-Nevanlinna representation to $d$-dimensional wedges $[34 ; 109] .{ }^{28}$ Let us first introduce Vladimirov's generalizations of the Poisson and Schwarz kernels, using the Cauchy kernel $\mathcal{K}_{C}$ from Eq. (5.28) as a starting point.

The (generalized) Poisson kernel for the wedge $T^{C}$ is defined by

$$
\mathcal{P}_{C}(\underline{z}):=\frac{\left|\mathcal{K}_{C}(\underline{z})\right|^{2}}{(2 \pi)^{d} \mathcal{K}_{C}(2 \underline{\mathrm{i}} \underline{y})} ; \quad \underline{z}=\underline{x}+\mathrm{i} \underline{y} .
$$

The (generalized) Schwarz kernel with respect to a point $\underline{z}^{(0)}=\underline{x}^{(0)}+\mathrm{i} \underline{y}^{(0)} \in T^{C}$ is given by

$$
\begin{array}{r}
\mathcal{S}_{C}\left(\underline{z}, \underline{z}^{(0)}\right):=\frac{2 \mathcal{K}_{C}(\underline{z}) \overline{\mathcal{K}_{C}\left(\underline{z}^{(0)}\right)}}{(2 \pi)^{d} \mathcal{K}_{C}\left(\underline{z}-\overline{\underline{z}^{(0)}}\right)} \\
-\mathcal{P}_{C}\left(\underline{x}^{(0)}, \underline{y}^{(0)}\right) .
\end{array}
$$

For a measure $\mu(\underline{x})$ we call

$$
P_{C}[\mathrm{~d} \mu](\underline{z}):=\int \mathrm{d} \mu(\underline{x}) \mathcal{P}_{C}(\underline{z}-\underline{x})
$$

the Poisson integral with respect to $\mu$.

\subsubsection{Vladimirov's Theorem}

We already pointed out that in the case $d \geq 3$ two arbitrary different wedges, $T^{C}$ and $T^{C^{\prime}}$, are usually not biholomorphically equivalent. In particular, the wedge $T^{C}$ is not necessarily biholomorphically equivalent to $\mathbb{H}^{d}$ when $d \geq 3$. Hence, one may expect the structural similarity to the Carathéodory functions to break down more easily in higher dimensions.

This is the reason why Vladimirov's $d$-dimensional generalization of Herglotz' theorem is stated in the following way [34]:

Theorem. (Vladimirov, 1978/79) The following conditions for a function $f \in$ $H_{+}\left(T^{C}\right)$ are equivalent for a cone $C \subset \mathbb{R}^{d}$ and $\mu(\underline{x}):=\operatorname{Im} f\left(\underline{x}+\mathrm{i} 0^{C}\right)$ :

1. The Poisson integral $P_{C}[\mathrm{~d} \mu]$ is pluriharmonic in $T^{C}$;

28 Vladimirov's approach may be found for positive real parts in Ref. [109] and for positive imaginary parts in Ref. [34]. 
2. the function $\operatorname{Im} f(\underline{z}), \underline{z}=\underline{x}+\mathrm{i} \underline{y} \in T^{C}$, is represented by the Poisson formula

$$
\operatorname{Im} f(\underline{z})=P_{C}[\mathrm{~d} \mu](\underline{z})+(\underline{a}, \underline{y}),
$$

for some $\underline{a} \in C^{*}$, where $C^{*}$ is the dual cone of $C$;

3. for all $\underline{z}^{0} \in T^{C}$, under the assumption that $C$ is regular, the Schwarz representation

$$
\begin{aligned}
f(\underline{z})= & \mathrm{i} \int_{\mathbb{R}^{d}} \mathcal{S}_{C}\left(\underline{z}-\underline{t}, \underline{z}^{0}-\underline{t}\right) \mathrm{d} \mu(t) \\
& +(\underline{a}, \underline{z})+\underline{b}
\end{aligned}
$$

holds, with $b=b\left(\underline{z}^{0}\right)=\operatorname{Re} f\left(\underline{z}^{0}\right)-\left(\underline{a}, \underline{x}^{0}\right)$.

Pluriharmonic functions are the natural multidimensional generalization of harmonic functions. A regular cone $C$ in our context is a cone for which $1 / \mathcal{K}_{C}$ is non-singular in $T^{C}$. In the cases $d=1,2,3$ all pointed cones are regular [109].

The first statement of the theorem is true for our type of domains, because it is known from the literature (see p. 134 in Ref. [109]) that the Poisson integral is pluriharmonic for any function $H_{+}\left(\mathbb{H}^{d}\right)$. Due to the biholomorphic equivalence of all $T^{C}$ to $\mathbb{H}^{2}$ in $\mathbb{C}^{2},{ }^{29}$ the two integral representations provide exact relations for all holomorphic sheets of the interacting Green's function. ${ }^{30}$ A parametrization of the Green's function with respect to their Edge values is gained by this. Due to the far-reaching biholomorphic equivalences, the validity of our representation has first been shown for the polycylinder by Korányi and Pukánsky [114].

Note that the linear growth term $\underline{a}$ is zero for the Green's function, because it is bounded, as required by assumption (5.13).

\subsection{Application to the Green's function}

It turns out to be reasonable to specify a given cone domain arising from the branch cut structure by an angle $\vartheta$ and an opening ratio $r$. See figure 5.4. It is sufficient to consider the case $\vartheta=0$ first, because relations for finite $\vartheta$ may be reconstructed from biholomorphic rotations, as explained in section 5.4.2.

\footnotetext{
${ }^{29}$ See the discussion of the biholomorphic transformations in section 5.4.5.

${ }^{30}$ It may again be helpful to recall that the holomorphic sheets are defined by subsequent branch cuts, as depicted in figure 3.4 .
} 


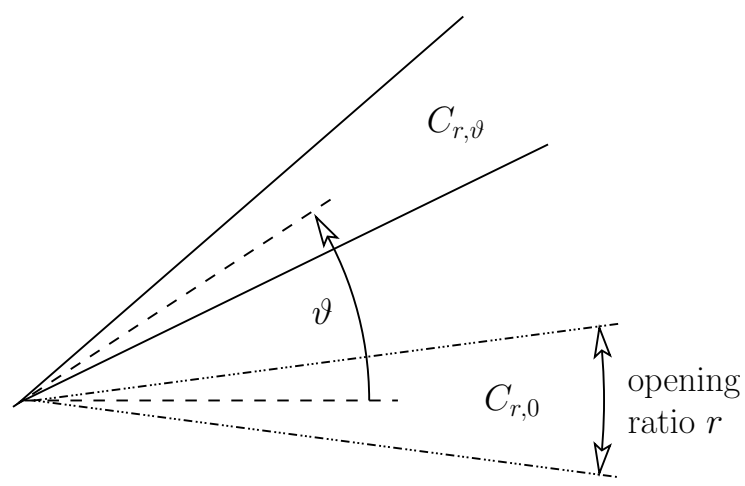

Figure 5.4: An arbitrary cone $C$ with 0 as vertex can be parametrized by an opening ratio $r$ and an orientation angle $\vartheta$. The rotation $R_{\vartheta}$ which maps $C_{r, 0}$ to $C_{r, \vartheta}$ induces a biholomorphism between $T^{C_{r, 0}}$ and $T^{C_{r, \vartheta}}$.

\subsubsection{Kernels for $\vartheta=0$}

For the case $\vartheta=0$, the computation of the kernels $\mathcal{K}_{C_{r, \vartheta}}$ and $\mathcal{P}_{C_{r, \vartheta}}$ can be accomplished straightforwardly. Using the definition

$$
C_{r, \vartheta}:=\bigcup_{\lambda \in(-r, r)}\left\{\left(x_{1}, x_{2}\right) \in \mathbb{R}^{2} \mid x_{2}>0 \wedge x_{1}=\lambda x_{2}\right\}
$$

one can compute the Cauchy and Poisson kernels via equations (5.28) and (5.38). The resulting Cauchy kernel is

$$
\mathcal{K}_{C_{r, 0}}(\underline{z})=-2 r \prod_{\mu= \pm 1} \frac{1}{r z_{2}-\mu z_{1}},
$$

and the resulting Poisson kernel is

$$
\mathcal{P}_{C_{r, 0}}(\underline{z})=\frac{r}{\pi^{2}} \prod_{\mu= \pm 1} \frac{r y_{2}-\mu y_{1}}{\left(r x_{2}-\mu x_{1}\right)^{2}+\left(r y_{2}-\mu y_{1}\right)^{2}} .
$$

An explicit use of the Schwarz kernel (5.39) seems to be less advantegeous, because the occurance of the reference point $\underline{z}^{(0)}$ appears to introduce additional technical complications.

\subsubsection{Operator Notation}

In order to put a stronger emphasize on the functional-analytic nature of the integral representations which interrelate edge and wedge values of the Green's function, let us introduce an operator notation for the Poisson integral and also for the biholomorphic 
rotations. Let us denote the set of all a-priori admitted Green's functions on $T^{C}$ by $\mathcal{G}_{C}$. By "a-priori admitted" we mean those analytic functions $\left.G\right|_{T^{C}}: T^{C} \rightarrow \mathbb{C}$ for which axiom 2 from section 5.3 holds ( $T^{C}$ has to comply with axiom 1$)$. Furthermore, let us denote the corresponding space of boundary value distributions $\left.G\right|_{T^{C}}\left(\underline{x}+\mathrm{i} 0^{C}\right)$ by $\mathcal{E}_{C}$. In order to focus on the Poisson kernel, we introduce the corresponding spaces of imaginary parts, $\mathcal{G}_{C}^{(I)}$ and $\mathcal{E}_{C}^{(I)}$.

We denote by the operator $\mathcal{P}_{C}$ the linear map

$$
\begin{aligned}
\mathcal{P}_{C}: \mathcal{E}_{C}^{(I)} & \rightarrow \mathcal{G}_{C}^{(I)}, \\
\operatorname{Im} G\left(\underline{x}+\mathrm{i} 0^{C}\right) & \mapsto \int \mathrm{d} x^{2} \mathcal{P}_{C}(\underline{z}-\underline{x}) \operatorname{Im} G\left(\underline{x}+\mathrm{i} 0^{C}\right) .
\end{aligned}
$$

Note that the Schwarz kernel (5.39) does not directly yield a comparable map from $\mathcal{E}_{C}$ to $\mathcal{G}_{C}$, due to the occurance of the $\underline{z}^{(0)}$ reference point.

Furthermore, the rotation $R_{\vartheta}$, which maps the cone $C_{r, 0}$ to the cone $C_{r, \vartheta}$, induces a biholomorphic map $\tilde{R}_{\vartheta}: T^{C_{r, 0}} \rightarrow T^{C_{r, \vartheta}}$ (see section 5.4.2). This biholomorphism maps functions $f \in \mathcal{G}_{C_{r, 0}}$ to functions $f \in \mathcal{G}_{C_{r, \vartheta}}$. This can be similarly represented by the linear operator

$$
\begin{aligned}
\mathcal{R}_{\vartheta}: \mathcal{G}_{C_{r, 0}} & \rightarrow \mathcal{G}_{C_{r, \vartheta}}, \\
f(\underline{z}) & \mapsto f\left(\tilde{R}_{\vartheta}^{-1}(\underline{z})\right) .
\end{aligned}
$$

The operator $\mathcal{R}_{\vartheta}$ also naturally extends to a linear map from $\mathcal{E}_{C_{r, 0}}$ to $\mathcal{E}_{C_{r, \vartheta}}$ which we will denote by the same symbol $\mathcal{R}_{\vartheta}$.

\subsubsection{Kernel functions at finite $\vartheta$}

Consequently, for finite $\vartheta$, the Poisson kernel operator of $T^{C_{r, \vartheta}}$ is

$$
\mathcal{P}_{C_{r, \vartheta}}=\mathcal{R}_{\vartheta} \mathcal{P}_{C_{r, 0}} \mathcal{R}_{\vartheta}^{-1}
$$

Equivalently, the Poisson kernel function of $T^{C_{r, \vartheta}}$ is given by

$$
\mathcal{P}_{C_{r, \vartheta}}(\underline{z})=\mathcal{P}_{C_{r, 0}}\left(R_{\vartheta}^{-1} \cdot \underline{z}\right) .
$$

In practical computations, the function can be evaluated combining equation (5.45) and the rotation matrix

$$
R_{\vartheta}^{-1}=\left(\begin{array}{cc}
\cos \vartheta & -\sin \vartheta \\
\sin \vartheta & \cos \vartheta
\end{array}\right)
$$




\subsubsection{Edge Properties of $G_{0}$}

Since we essentially reduced the structure of the Green's function to the edge values of their holomorphic branches, it seems worthwhile to investigate the edge structure of $G_{0}$, and later also the perturbative structure of the theory in $U$, more carefully. See section 7.1 for the deeper analysis.

The edge limit of the bare Green's function (5.9), as a function of the edge orientation $\vartheta$, is given by

$$
G_{0}^{(\text {edge })}(\vartheta ; \underline{x})=\sum_{\alpha= \pm 1} G_{0}^{(\text {edge }), \alpha}(\vartheta ; \underline{x})
$$

where

$$
G_{0}^{(\mathrm{edge}), \alpha}(\vartheta ; \underline{x})=\frac{\Gamma_{\alpha} / \Gamma}{x_{\omega}-\alpha\left(x_{\varphi}-\Phi\right) / 2-\varepsilon_{d}+\mathrm{i} \Gamma \operatorname{sgn}_{\vartheta}}
$$

and

$$
\operatorname{sgn}_{\vartheta}:=\operatorname{sgn}\left(\cos \vartheta-\frac{\alpha}{2} \sin \vartheta\right) .
$$

Apparently, the edge function only changes as a function of $\vartheta$ whenever $\cos \vartheta \pm$ $\sin \vartheta / 2$ crosses zero. This reflects the equivalence of all directions

$$
\underline{x}+\mathrm{i} 0^{\vartheta}:=\underline{x}+\mathrm{i} \cdot\left(\begin{array}{c}
\sin \vartheta \\
\cos \vartheta
\end{array}\right) 0^{+}
$$

when approaching the edge within a holomorphic branch $T^{C}$, as discussed in section 5.4.2. The edge function changes whenever $\vartheta$ crosses a branch cut, namely for the following singular orientations in the interval $[0,2 \pi)$ :

$$
\begin{aligned}
& \vartheta_{1}^{(\text {sing })}=\arctan 2 \\
& \vartheta_{2}^{(\text {sing })}=\pi-\arctan 2 \\
& \vartheta_{3}^{(\text {sing })}=\pi+\arctan 2 \\
& \vartheta_{4}^{(\text {sing })}=2 \pi-\arctan 2 .
\end{aligned}
$$

The orientations $\vartheta_{i}^{(\text {sing })}$ are also identical to the singular directions of assumption 3' in section 5.3.

There is another subtle feature of the edge behaviour of the bare Green's function. The real part

$$
\operatorname{Re} G_{0}^{(\text {edge })}(\vartheta ; \underline{x})=\sum_{\alpha= \pm 1} \frac{\frac{\Gamma_{\alpha}}{\Gamma}\left(x_{\omega}-\alpha\left(x_{\varphi}-\Phi\right) / 2-\varepsilon_{d}\right)}{\left(x_{\omega}-\alpha\left(x_{\varphi}-\Phi\right) / 2-\varepsilon_{d}\right)^{2}+\Gamma^{2}}
$$

is completely $\vartheta$-independent. As a consequence, for any branch of $G_{0}$, the edge limit $\operatorname{Re} G_{0}\left(\underline{x}+\mathrm{i} 0^{C}\right)$ is identical. 
Another property is related to the physical limit (5.11) of the Green's function. With regard to the orientation within the branch cut structure of the respective limiting procedure, one obtains that $\vartheta=0$ corresponds to the retarded Green's function. One finds that the quantity

$$
\tilde{A}_{0}(\underline{x}):=-\frac{1}{\pi} \operatorname{Im} G_{0}\left(\underline{x}+\mathrm{i} \cdot 0^{\vartheta=0}\right),
$$

where $\underline{x}+\mathrm{i} \cdot 0^{\vartheta=0}$ is defined according to Eq. (5.55), is positive definite:

$$
\tilde{A}_{0}(\underline{x})=\sum_{\alpha= \pm 1} \frac{\Gamma_{\alpha} / \pi}{\left(x_{\omega}-\alpha\left(x_{\varphi}-\Phi\right) / 2-\varepsilon_{d}\right)^{2}+\Gamma^{2}} .
$$

Within the MaxEnt method employed in the following chapters, this will be of use in the probabilitic interpretation of the fit variables associated to the integration space.

In particular, the non-interacting spectral function

$$
A_{0}(\omega)=\tilde{A}_{0}(\Phi, \omega) .
$$

Again one can see in equation (5.59) that $\tilde{A}_{0}$ does not decay to zero as a function of $\underline{x} \rightarrow \infty$ along the singular directions $\vartheta_{i}^{(\text {sing })}\left(\underline{x}^{(0)}\right.$ in axiom 3', section 5.4.2). This is because the occurance of singular directions is an essential feature of the edges and the holomorphic structure of the Green's function. It leads to a discontinuity at $\infty .{ }^{31}$

\subsection{Summary}

In the present chapter, basic notions of the theory of several complex variables were introduced. It was pointed out that the Green's functions of Matsubara voltage theory have holomorphic branches living on wedge domains which are in mathematical terminology the well-known tubular cone domains. They represent a generalization of the upper half plane to two or more complex variables. The analytic properties of the Green's function within a wedge were characterized. An integral representation for an arbitrary analytic branch of the Green's function for a wedge was derived. By this, a linear relation between imaginary-voltage data and the edge of the wedge was achieved. Since it establishes a link between real-time and imaginary-time structure of the theory, it will be utilized by the numerical analytic continuation procedures in the next two chapters.

\footnotetext{
${ }^{31}$ See the compactification discussed in chapter 5.4.5 and in particular figure 5.3.
} 


\section{Bayesian Inference for Numerical Analytic Continuation}

In order to analytically continue the imaginary-time quantum Monte-Carlo simulation data, a rather involved numerical technique has to be invoked. This so-called MaxEnt algorithm [35] provides Bayesian estimates on real-time properties based on evidence given by the imaginary-time simulation data. The Bayesian estimates are necessary because real-time and imaginary-time structure are related via an integral equation whose inversion is numerically ill-posed. As consequence, the stochastic noise associated to the Monte-Carlo simulation is amplified by the numerical inversion. The inversion has to be regularized appropriately, in order to obtain physically insightful data from the simulation. The MaxEnt incorporates a-priori information about the real-time structure via Bayes' theorem in order to accomplish this task.

In the example of conventional equilibrium bosonic or fermionic correlation functions one has the representation [97]

$$
G\left(\mathrm{i} \omega_{n}\right)=\int \frac{A(\varepsilon)}{\mathrm{i} \omega_{n}-\varepsilon} \mathrm{d} \varepsilon=: K_{\mathrm{eq}}[A]\left(\omega_{n}\right),
$$

where $G\left(\mathrm{i} \omega_{n}\right)$ is the Gaussian random variable associated to the output of a thorough quantum Monte-Carlo simulation at bosonic or fermionic Matsubara frequency $\omega_{n}$. An optimal estimator for the spectral function $A(\varepsilon)$ is needed, given some measured mean values $\bar{G}$ and their covariance matrix $C$. A simple least-square estimator identifying a function $A$, with $\chi^{2}[A] \approx N$, given $N$ QMC data points, is not of any practical use, because it can be shown that a vast amount of qualitatively different functions with this property exists. ${ }^{1}$ This is the ill-posed nature of the analytic continuation problem of quantum Monte-Carlo data.

The standard approach to understand and partially resolve this issue is the MaxEnt method. Based on Bayesian inference, ${ }^{2}$ it provides a language to systematically incorporate the information provided by QMC data into a-priori known facts about the spectral function. In the fermionic case, $A(\omega)$ is a positive semidefinite function and can be interpreted as a probability density. The additional information is then

\footnotetext{
${ }^{1}$ We use the definition $\chi^{2}:=\sum_{k, l=1}^{N}\left(\bar{G}_{k}-(K A)_{k}\right) C^{-1}\left(\bar{G}_{l}-(K A)_{l}\right)$, where $K$ is the analytic continuation kernel.

2 An excellent introduction to the algebra of probabilistic inference, which may be regarded as a generalization of Boolean algebra to random variables, is provided by Ref. [115].
} 
introduced by a so-called default model $D(\omega)$, defining an entropic prior. The entropic prior can be derived with Bayes' theorem, introducing the entropy [116]

$$
S[A, D]=\int \mathrm{d} \varepsilon\left(A(\varepsilon)-D(\varepsilon)-A(\varepsilon) \log \frac{A(\varepsilon)}{D(\varepsilon)}\right)
$$

If it is known that the $\bar{G}$ data do not contain any systematic error, the exact constraint $\int A(\varepsilon) \mathrm{d} \varepsilon=1$ yields via Bayesian $\operatorname{logic}^{3}$ the entropy

$$
S^{\mathrm{norm}=1}[A, D]=-A(\varepsilon) \log \frac{A(\varepsilon)}{D(\varepsilon)} .
$$

For Hirsch-Fye input data, the choice (6.2) is standard for practical reasons, although the normalization is known exactly [35]. The entropy (6.2) is the optimal choice in cases in which function $A(\varepsilon)$ is a positive definite function with unknown normalization. The entropic prior has the form $\operatorname{Pr}[A] \propto \mathrm{e}^{\alpha S}$, where $\alpha$ is some scale factor. In order to introduce the information contained in the stochastic data, the functional

$$
Q:=\alpha S-\frac{1}{2} \chi^{2}
$$

is maximized with respect to $A$ in MaxEnt. In order to obtain results which are independent of the choice of the hyperparameter $\alpha$, several approaches exist. The simplest approach, so-called historic MaxEnt, chooses the $\alpha$ for which $\chi^{2}=N$. Historic MaxEnt is, however, not considered to be optimal with regard to the ultimate aim of taking account of all information contained in the data. It merely tends to ignore some valuable information.

From a Bayesian point of view, a better approach with this respect are classic and Bryan's MaxEnt. They are based on the computation of the posterior probability

$$
\operatorname{Pr}[\alpha \mid \bar{G}, D]=\operatorname{Pr}[\alpha] \int \mathcal{D} A \frac{\mathrm{e}^{Q}}{Z_{l} Z_{s}(\alpha)}
$$

Because $\alpha$ is a scaling factor, the prior $\operatorname{Pr}[\alpha] \propto \alpha^{-1}$ (Jeffreys prior) [117]. The terms $Z_{l}$ and $Z_{s}(\alpha) \propto \alpha^{-N / 2}$ are some partition sums. A sharp maximum of $\operatorname{Pr}[\alpha \mid \bar{G}, D]$ is obtained in practical computations if $N$ is large [118; 119]. Classic MaxEnt chooses the $A$ which maximizes $\operatorname{Pr}[\alpha \mid \bar{G}, D]$. Bryan's MaxEnt computes the weighted average $\int \mathrm{d} \alpha \operatorname{Pr}[\alpha \mid \bar{G}, D] A^{(\alpha)}$. While classic and Bryan's MaxEnt are, from a Bayesian point of view, better estimates, a good check for QMC data quality and significance of inferred results is the deviation between historic, classic, and Bryan's MaxEnt.

The standard MaxEnt implementation for the analytic continuation problem [35] uses the algorithm by Bryan [118]. It is particularly useful, because the algorithm is

\footnotetext{
${ }^{3}$ It can readily be derived from Eq. (6.2), also.
} 
optimized for oversampled data sets. The ill-posed problem of analytic continuation is in fact oversampled, because it is comparably cheap to generate a vast amount of imaginary-time data at different imaginary times or frequencies by QMC measurements which at some point, which is to be qualified, do not contribute any additional information.

While the application of MaxEnt to the continuation problem is almost excessively discussed in Ref. [35], we will have to comment on the implementation used in this work. It is found practical to go beyond the description of Bryan's algorithm given in Ref. [35] and implement the optimizations specified by Bryan in his original paper, Ref. [118]. The optimizations are very effective for the vast amount of imaginary-time data which come along with the imaginary-voltage formalism. The central trick is a singular value decomposition (SVD) of the kernel

$$
K: V_{\tilde{A}} \rightarrow V_{\text {data }}, \quad K=V \Sigma U^{T},
$$

with $V, U^{T}$ orthogonal, and the singular values

$$
\Sigma=\operatorname{diag}\left(\sigma_{1}, \sigma_{2}, \ldots, \sigma_{s}, 0, \ldots, 0\right),
$$

$\sigma_{1} \geq \sigma_{2} \geq \cdots \geq \sigma_{s}>0$. Many important quantities may be reduced to the $s$ dimensional singular space $V_{\Sigma}$. Most notably, the $\left(\operatorname{dim} V_{\tilde{A}}\right)$-dimensional optimization problem given by

$$
Q[\tilde{A}] \stackrel{!}{=} \min
$$

may be solved within the singular space using Levenberg-Marquardt iterations. As $s$ is comparably small after truncating the singular space with respect to the floating point precision of the singular values $\sigma_{i}$, the algorithm is still sufficiently efficient.

In order to analytically continue data from different QMC simulations we use different seeds for the random number generators of each $\varphi_{m}$ value. It is assumed that observables gained for values $m \neq m^{\prime}$ are not correlated. Consequently, in order to estimate the covariance matrix $C$, a block-diagonal shape

$$
C=\left(\begin{array}{cccc}
C^{\left(m_{\min }\right)} & 0 & \cdots & \\
0 & C^{\left(m_{\min }+1\right)} & 0 & \ldots \\
\vdots & 0 & \ddots & \\
& \cdots & 0 & C^{\left(m_{\max }\right)}
\end{array}\right)
$$

is assumed whose blocks $C^{(m)}$ are estimated from the output of each individual $\varphi_{m}$-th QMC simulation. 


\subsection{Static Observables}

In this section, we will introduce the application of Maximum Entropy to the estimation of static quantities within Matsubara voltage theory. In contrast to equilibrium QMC computations, static observables cannot be measured directly but have to be analytically continued with respect to the single variable $i \varphi_{m}$. On top of this, for some observables, most prominently the current operator, it can be shown that the analytic continuation $\mathrm{i} \varphi_{m} \rightarrow \Phi$ does not yield the physical result. ${ }^{4}$ However, this is apparently possible for other types of observables. An important subset of these "good" observables appears to be comprised of those which only measure correlations between dot degrees of freedom. It has been shown that functions of $n_{d, \sigma}$ are observables of this kind. Details will be published in Ref. [54]. Consequently, dot magnetization

$$
m=\left\langle d_{\uparrow}^{\dagger} d_{\uparrow}\right\rangle-\left\langle d_{\downarrow}^{\dagger} d_{\downarrow}\right\rangle
$$

and double occupancy

$$
d=\left\langle d_{\uparrow}^{\dagger} d_{\uparrow} d_{\downarrow}^{\dagger} d_{\downarrow}\right\rangle
$$

are good observables. It has been shown that static observables are holomorphic on the upper and lower half plane (cf. section 3.4.5). As derived below, for an observable $\mathcal{O}$ which is either double occupancy or magnetization, one finds that a representation

$$
\langle\mathcal{O}\rangle\left(\mathrm{i} \varphi_{m}\right)=\langle\mathcal{O}\rangle_{\text {const }}+\int \frac{A_{\mathcal{O}}(\varphi)}{\left(\mathrm{i} \varphi_{m}-\Phi\right)-\varphi} \mathrm{d} \varphi
$$

holds, where $\langle\mathcal{O}\rangle_{\text {const }}$ is a real number, and $A_{\mathcal{O}}(\varphi) \in \mathbb{R}$ is a real-valued spectral function.

Once the validity of representation (6.12) is established, the implementation of a MaxEnt estimator for the physical expectation value is rather straightforward. After measuring the complex expectation values $\langle\mathcal{O}\rangle\left(\mathrm{i} \varphi_{m}\right)$ of the observable $\mathcal{O}$ with respect to the effective-equilibrium system with nonhermitian Hamiltonian at Matsubara voltage $i \varphi_{m}$, one has typically obtained a reliable estimate of the correlation matrix $C$ of all values. This is because due to the independence of the simulations, there is only the variance and correlation of imaginary and real parts of a single $i \varphi_{m}$ value to be estimated. Therefore, the typical problems which arise for the covariance estimation of Matsubara Green's function measurements, within a single QMC run, do not appear. $^{5}$ The constant $\langle\mathcal{O}\rangle_{\text {const }}$ can be eliminated before the MaxEnt procedure is involved into data analysis. In order to do so the high-energy tail of $\langle\mathcal{O}\rangle\left(\mathrm{i} \varphi_{m}\right)$ is fitted to $\langle\mathcal{O}\rangle_{\text {const }}+\frac{c}{\mathrm{i} \varphi_{m}}+\frac{d}{\left(\mathrm{i} \varphi_{m}\right)^{2}}+\cdots$. In practice, the weighted least-square fit yields reliable values and error bars for $\langle\mathcal{O}\rangle_{\text {const }}$. Via Gaussian error propagation it is then

\footnotetext{
${ }^{4}$ See section 3.4.1.

${ }^{5}$ Covariance estimation issues are discussed in more detail in the discussion of dynamic expectation values, section 6.2 .
} 


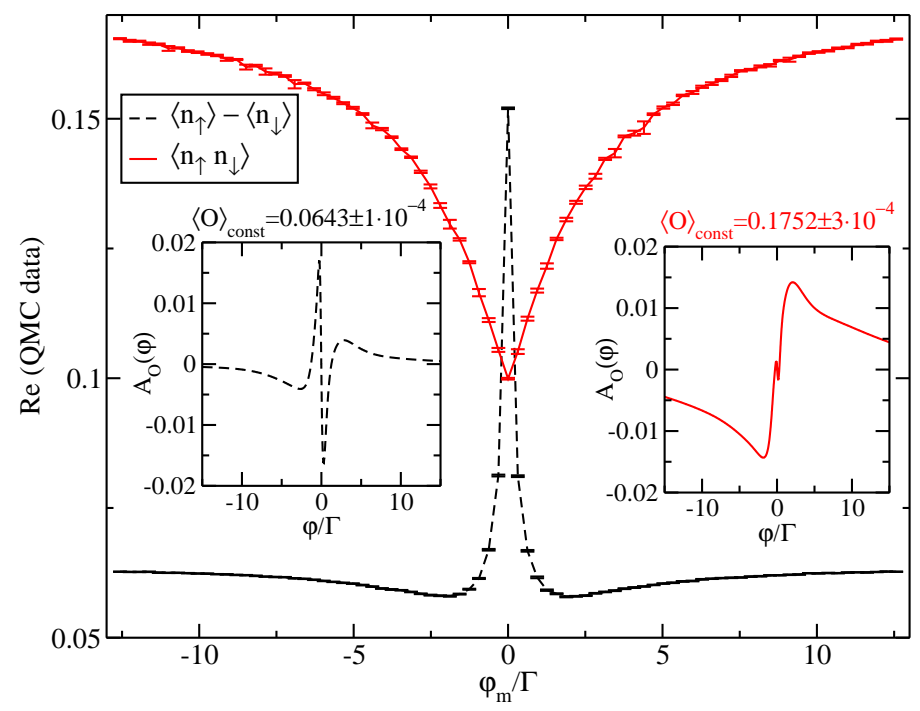

Figure 6.1: Simulated QMC data and inferred spectral functions at $U=8 \Gamma, e \Phi=0.01 \Gamma$, $\beta=40.0 \Gamma^{-1}$, and the magnetic field $\mu_{B} B=0.02 \Gamma$.

possible to incorporate the uncertainty of $\langle\mathcal{O}\rangle_{\text {const }}$ into the covariance matrix of the quantity $\langle\mathcal{O}\rangle\left(\mathrm{i} \varphi_{m}\right)-\langle\mathcal{O}\rangle_{\text {const. }}{ }^{6}$

Because the MaxEnt method is only applicable to the inference of positive definite functions, a shift function $A_{\text {shift }}(\varphi)$ of the spectral function $A_{\mathcal{O}}(\varphi)$ has to be introduced, which makes the to-be-inferred $A_{\mathcal{O}}^{\prime}(\varphi)=A_{\mathcal{O}}(\varphi)-A_{\text {shift }}(\varphi)$ positive. We employ a symmetry condition

$$
A_{\text {shift }}(\varphi)=A_{\text {shift }}(-\varphi)
$$

because this choice is robust with respect to the physical result ${ }^{7}$

$$
\langle\mathcal{O}\rangle_{\text {phys }}=\frac{1}{2} \sum_{\alpha= \pm 1}\langle\mathcal{O}\rangle\left(\Phi+\alpha \mathrm{i}^{+}\right)=\langle\mathcal{O}\rangle_{\text {const }}-\not \oint \mathrm{d} \varphi \frac{A_{\mathcal{O}}(\varphi)}{\varphi} .
$$

This is due to the invariance of $\langle\mathcal{O}\rangle_{\text {phys }}$ under symmetric modifications of $A_{\mathcal{O}}(\varphi)$. Using a particular $D_{\text {shift }}(\varphi)$, the data $\langle\mathcal{O}\rangle\left(\mathrm{i} \varphi_{m}\right)-\langle\mathcal{O}\rangle_{\text {const }}-\int \mathrm{d} \varphi \frac{A_{\text {shift }}(\varphi)}{\mathrm{i} \varphi_{m}-\Phi-\varphi}$ are used as input for the MaxEnt algorithm. Example input and output data of the inference process are shown in figure 6.1 for double occupancy and magnetization. The re-

\footnotetext{
${ }^{6}$ Note that corrections from error propagation to offdiagonal covariance matrix elements were neglected. This may be justified, because correlations between real and imaginary part of an effective-equilibrium expectation value are the only nonzero offdiagonal elements.

7 The principal value integral results from the symmetric averaging discussed in section 3.4. It generates the correct principal value integrals of the real-time expansion in the mapping from Matsubara voltage theory to Keldysh theory. Note that if upper or lower value would have a stronger weight, the physical expectation value would be complex, which is, of course, unphysical.
} 
sulting MaxEnt spectra are shown, together with the previously estimated constant offset term.

\subsubsection{Double Occupancy}

We will first show that the representation (6.12) holds for the double occupancy,

$$
d\left(\mathrm{i} \varphi_{m}\right)=d_{0}+\int \frac{A_{\mathrm{docc}}(\varphi)}{\mathrm{i} \varphi_{m}-\Phi-\varphi} \mathrm{d} \varphi
$$

We restrict to the case of particle-hole symmetry and symmetric coupling to the leads, $\Gamma_{L}=\Gamma_{R}$. Wick's theorem yields that

$$
d\left(\mathrm{i} \varphi_{m}\right)=\left\langle n_{\uparrow}\right\rangle\left\langle n_{\downarrow}\right\rangle+\frac{1}{\beta U} \sum_{\omega_{n}} \Sigma\left(\mathrm{i} \varphi_{m} ; \mathrm{i} \omega_{n}\right) G\left(\mathrm{i} \varphi_{m} ; \mathrm{i} \omega_{n}\right) \mathrm{e}^{\mathrm{i} \omega_{n} 0^{+}} .
$$

Due to particle-hole symmetry, we have $\left\langle n_{\uparrow}\right\rangle\left\langle n_{\downarrow}\right\rangle=1 / 4$. Obviously, the $1 / \mathrm{i} \varphi_{m}$ decay of Green's function and selfenergy allow for the existence of the spectral representation (6.15), as long as there is only a single branch cut at $\operatorname{Im} z_{\varphi}=0$.

The real-valuedness of spectral function and constant offset remain to be shown. The general relation $G\left(-\mathrm{i} \varphi_{m},-\mathrm{i} \omega_{n}\right)^{*}=G\left(\mathrm{i} \varphi_{m}, \mathrm{i} \omega_{n}\right)$ holds for Green's function and selfenergy, see section 3.4.4. Inserting into Eq. (6.16), we find

$$
d\left(-\mathrm{i} \varphi_{m}\right)^{*}=d\left(\mathrm{i} \varphi_{m}\right) .
$$

Consequently, the real part of $d\left(\mathrm{i} \varphi_{m}\right)-d\left(-\mathrm{i} \varphi_{m}\right)$ vanishes. Using the symmetric coupling to the leads, we have an invariance of the Green's function and selfenergy under $\left(\mathrm{i} \varphi_{m}-\Phi\right) \leftrightarrow-\left(\mathrm{i} \varphi_{m}-\Phi\right)$. Consequently, $d_{0}$ is an actual, sheet-independent constant. Due to the symmetry of $\operatorname{Im} d\left(\mathrm{i} \varphi_{m}\right), d_{0}$ is real. By inserting the representation (6.15) into (6.17) we see that $A_{\text {docc }}(\omega)$ is real-valued.

Figure 6.2 shows computed double occupancy data as compared to scatteringstates numerical renormalization group (SNRG) [22], second-order perturbation theory (PT), and real-time quantum Monte-Carlo (RT-QMC) [32]. ${ }^{8}$ The "classic" MaxEnt estimates seem to be subject to more statistical fluctuations than the "historic" MaxEnt, presumably because the "classic" estimator is designed to overfit data $\left(\chi^{2} / N_{\text {data }}<1\right)$ in favor of information which is supposed to be contained in the default model. The error bars are not actual error bars, because an arbitrarily informative default model can generate arbitrary physical expectation values via the MaxEnt process. We rather use a set of symmetric flat shift functions. The error bars denote the range over which resulting expectation values are scattered. ${ }^{9}$ The

\footnotetext{
${ }^{8}$ SNRG data were provided by Sebastian Schmitt and Frithjof Anders. Real-time QMC data were provided by Philipp Werner. Used with permission.

${ }^{9}$ Further details of this procedure will be published in Ref. [54].
} 


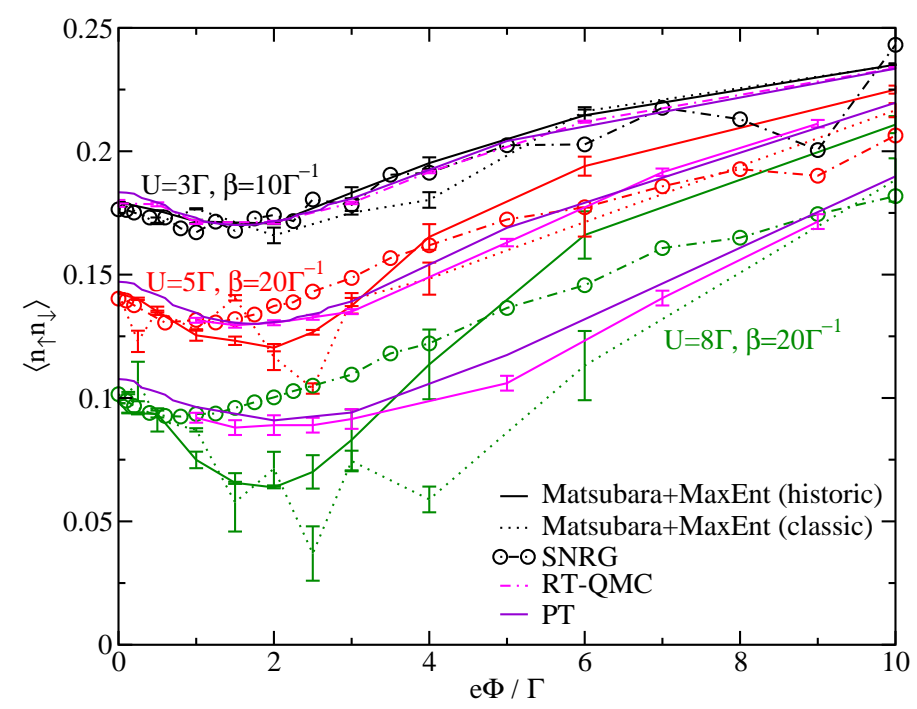

Figure 6.2: Double occupancy as a function of the bias voltage for "historic" and "classic" estimators over a broad range of the bias voltage. Data are compared to second-order perturbation theory (PT), scattering-states NRG (SNRG), and real-time quantum MonteCarlo (RT-QMC).

default model is chosen to be flat, because no a-priori information is available.

An excellent agreement between SNRG and our method is obtained for small charging energy $U=3 \Gamma$ and also for any $U$ at small bias voltages $e \Phi<0.5 \Gamma$. At larger bias voltages, the effective-equilibrium systems associated to the high-energy tail needed to estimate $d_{0}$ increasingly suffer from a phase problem, which makes the MaxEnt estimates less accurate. Nevertheless, also for the range $e \Phi>0.5 \Gamma$, the estimated curves for the double occupancy look reasonable. The Matsubara voltage approach appears to predict a deeper minimum at larger voltage for the double occupancy, as compared to SNRG. For all curves, at large biases, the non-interacting value of 1/4 is approached.

All methods agree on the prediction with regard to interaction strength $U=3 \Gamma$. Interestingly, SNRG disagrees with the other methods with regard to the position of the minimum for $U \geq 5 \Gamma$. While real-time QMC tends to agree with second-order perturbation theory, our MaxEnt estimates give rise to more pronounced minima, but apart from that a good agreement with real-time QMC is obtained. Source of the deviation may be the flat default model.

\subsubsection{Magnetic Susceptibility}

An observable which is much more sensitive to the Kondo effect is the magnetic susceptibility of the quantum dot, because it directly probes the destruction of the Kondo singlet. In equilibrium, a strong dependence on the temperature is observed, 


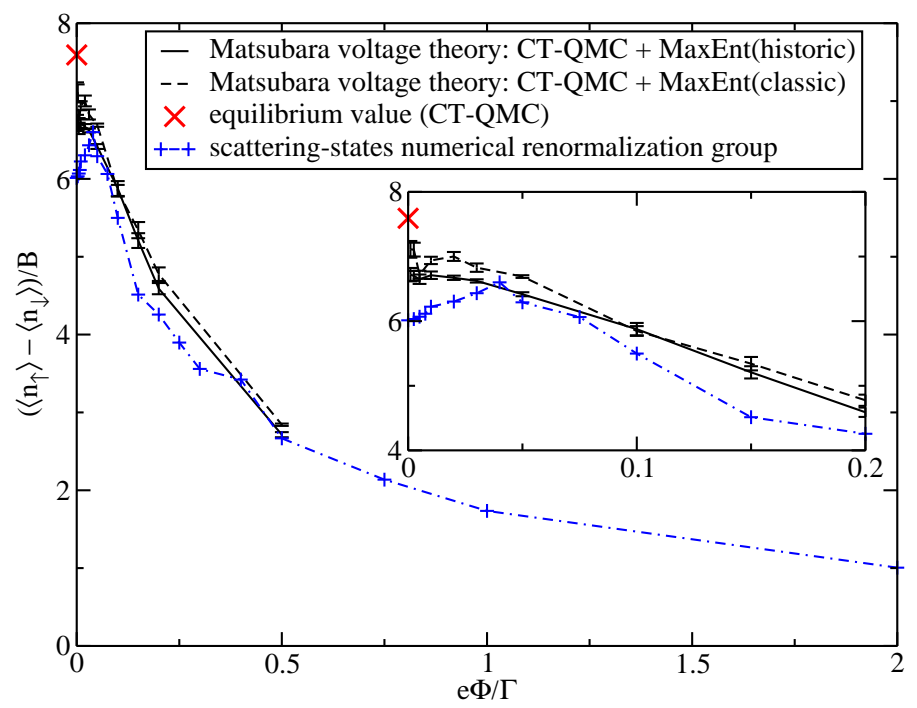

Figure 6.3: Magnetic susceptibility as a function of the bias voltage for $U=8 \Gamma, \mu_{B} B=$ $0.02 \Gamma, \beta=40 \Gamma^{-1}$ compared to scattering-states numerical renormalization group.

on the scale of the Kondo temperature [6].

The validity of a spectral representation

$$
m\left(\mathrm{i} \varphi_{m}\right)=m_{0}+\int \frac{A_{\text {magn }}(\varphi)}{\mathrm{i} \varphi_{m}-\Phi-\varphi} \mathrm{d} \varphi
$$

can readily be confirmed. Starting from the symmetry $G\left(-\mathrm{i} \varphi_{m},-\mathrm{i} \omega_{n}\right)^{*}=G\left(\mathrm{i} \varphi_{m}, \mathrm{i} \omega_{n}\right)$, one can again show that $m\left(-\mathrm{i} \varphi_{m}\right)^{*}=m\left(\mathrm{i} \varphi_{m}\right)$, and the same arguments which were used for the double occupancy apply, concerning the interchange $\left(\mathrm{i} \varphi_{m}-\Phi\right) \leftrightarrow$ $-\left(\mathrm{i} \varphi_{m}-\Phi\right)$.

As can be expected from the equilibrium case, our nonequilibrium results show a strong dependence on the voltage within the Kondo scale. ${ }^{10}$ This is shown in figure 6.3. An excellent agreement with the scattering-states numerical renormalization group is found. The MaxEnt results are biased from below, by construction, because the default value of the "classic" and "historic" estimators is $\langle\mathcal{O}\rangle_{\text {const }}$, due to the flat default model. A further analysis of SNRG data indicates that the breakdown at small voltages is an artifact of the method. ${ }^{11}$ The biasedness of the MaxEnt estimates as compared to the equilibrium value is less drastic for the Matsubara-voltage theory. Hence, our method seems to be a promising computational tool for the study of the Kondo effect out of equilibrium.

\footnotetext{
${ }^{10}$ The resolved energy scale is in fact the Kondo scale and not the energy scale associated to the magnetic field. This was checked by a qualitative scaling analysis at different values of $U$ at same values of $e \Phi, \mu_{B} B$, and $k_{B} T$ in units of the equilibrium Kondo temperature $k_{B} T_{K}$ [54].

${ }^{11}$ Sebastian Schmitt, private conversation.
} 


\subsection{Dynamic Observables}

We will now discuss the estimation of dot electron spectral functions within Matsubara voltage theory. The computation of conductance data within the formalism crucially depends on the knowledge of the spectral function, because the current operator itself is not a "good" observable within the formalism (see section 3.4.1). Once the spectral function $A_{\sigma}(\omega)$ of the spin- $\sigma$ dot electron is known, the expectation value of the current operator is given by the Landauer-type expression by Meir and Wingreen [88]:

$$
\langle I\rangle=\frac{e \Gamma}{2 h} \sum_{\sigma} \int \mathrm{d} \varepsilon\left[f_{L}(\varepsilon)-f_{R}(\varepsilon)\right] A_{\sigma}(\varepsilon) .
$$

As a consequence, in order to succeed in computing conductance data within the formalism, a detailed understanding and controlled algorithmic consideration of the structure of the dot electron Green's function is mandatory.

Preceding calculations of conductance data within the Matsubara-voltage formalism rely on arbitrarily regularized fits to the functional form of the second-order selfenergy [27; 53]. It is not clear to which extent these fits yield reliable estimates. Nevertheless, a good agreement with other methods has been reported in the weak to intermediate coupling limit [53].

A main goal of the present work is thus to investigate systematically the applicability of standard Bayesian inference methods to the double analytic continuation problem which is obtained for the dot-electron Green's function. In order to do so, we use the exact relations from two-variable complex function theory which were derived in chapter 5 .

\subsubsection{Single-Wedge Continuation}

A naïve and straightforward attempt on applying Bayesian inference to the functiontheoretical structure of the Green's function is the following. We ignore the $\operatorname{Im} z_{\varphi}=0$ branch cut, ${ }^{12}$ indications for the mere existence of which were first pointed out in a comparably recent publication [53].

One can argue that, considering the first kind of branch cuts, given by $\operatorname{Im} z_{\omega}=$ $\frac{\gamma}{2} \operatorname{Im} z_{\varphi}$, Eq. (3.84), $\gamma$ odd, the $\gamma$-th branch cut first appears in approximately $\gamma$-th order of perturbation theory. Consequently, at finite temperature and finite $U / \Gamma$, there will exist a critical value $\gamma_{\text {crit }}$, for which first-kind branch cuts $\gamma>\gamma_{\text {crit }}$ effectively do not exist. At this point, the Green's function may be assumed to be analytic within the wedge comprised of the remaining area governed by the neglected branch cuts. This is sketched in figure 6.4. The shaded area is the wedge within which the Green's function is assumed to be analytic. Within the grid of Matsubara data, the crosses denote those data points which are taken into account and circles

\footnotetext{
${ }^{12}$ See "branch cut from vertex corrections" in figure 3.4 and discussion of equation (3.85).
} 


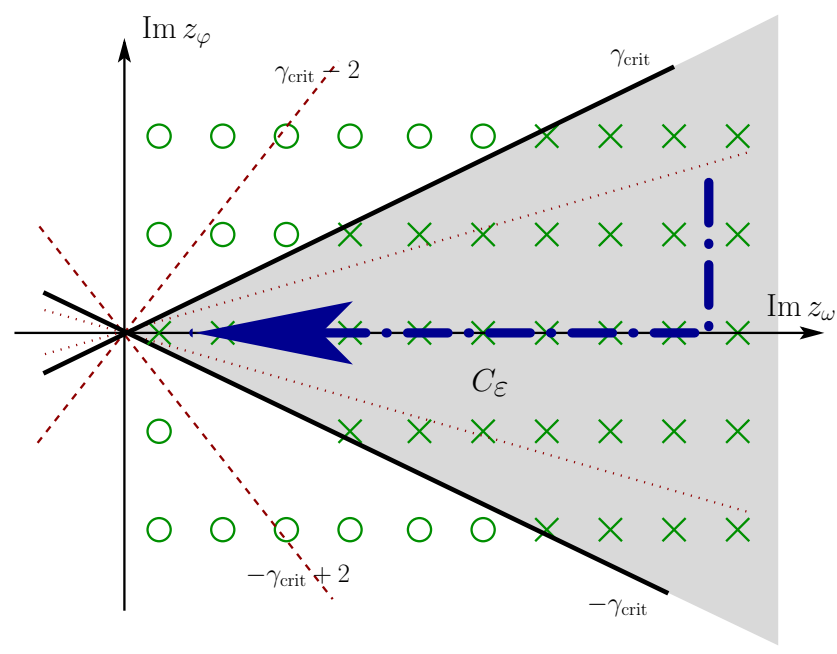

Figure 6.4: Geometry of the single-wedge continuation approach. All branch cuts within the $T^{C_{\varepsilon}}$ wedge are neglected. This can only be justified for the first-kind branch cuts, equation (3.84). The dot-electron spectral function is located on the edge of $T^{C_{\varepsilon}}$.

those which are not. The blue arrow denotes the direction of analytic continuation, which is compatible with the limiting procedure (5.11).

The resulting object of interest is a wedge $T^{C_{\varepsilon}}$. One can hence invoke Vladimirov's continuation kernel (5.45). We rename the opening ratio $r$ by $\varepsilon$. The positive definiteness of the function $\tilde{A}_{0}(\underline{x})$, see section 5.6.4, justifies the a-priori assumption that for the interacting Green's function $G$, the edge function

$$
\tilde{A}(\underline{x}):=-\left.\frac{1}{\pi} \cdot \operatorname{Im} G\right|_{\operatorname{Edge}_{T} C_{\varepsilon}}(\underline{x})
$$

is also positive definite. In principle, a violation of this assumption can be detected by the MaxEnt method.

\section{Computational Procedure}

In order to qualify data, default model, and solution quality, the strategies from Ref. [35] are adopted. A strategy to qualify the kernel function itself (i.e. a measure for the validity of the analyticity assumption for $T^{C_{\varepsilon}}$ ), is unfortunately not at hand. In principle, this would fall into the class of so-called blind deconvolution problems, which are far beyond the scope of this thesis [120]. Thus, in the present implementation, a small enough $\varepsilon$ is chosen a priori (and the existence of the $\operatorname{Im} z_{\varphi}=0$ branch cut is ignored).

Using the posterior probability distribution (6.5), a posterior for the default model 
$\tilde{D}(\underline{x})$ can be computed, based on the evidence of the QMC data, by

$$
\operatorname{Pr}(\tilde{D} \mid \bar{G}) \propto \int \mathrm{d} \alpha \int \mathcal{D} \tilde{A} \operatorname{Pr}(\alpha) \frac{\mathrm{e}^{Q}}{Z_{L} Z_{S}(\alpha)}
$$

In order to qualify the estimate of the covariance matrix $C$, one can test for a most probable mock rescaling $\sigma^{2}$ of $C$ [35]. The covariance is formally substituted by $\sigma^{2} C$. If the most probable $\sigma$, i.e. the solution of

$$
\frac{\chi_{\text {classic }}^{2}}{\sigma^{2}}+N_{g}=N
$$

deviates from 1 by more than a few tens of percent, the input data are rejected [35]. Here, $\chi_{\text {classic }}^{2}$ is the $\chi^{2}$ value of the classic MaxEnt solution, the number of data points $N$, and the number of "good" data points $N_{g}=\sum_{i} \frac{\lambda_{i}}{\alpha_{\text {classic }}+\lambda_{i}}$ with $\lambda_{i}$ the eigenvalues of

$$
\Lambda_{i j}=\left[\sqrt{\tilde{A}_{i}} \frac{\partial^{2} \chi^{2} / 2}{\partial \tilde{A}_{i} \partial \tilde{A}_{j}} \sqrt{\tilde{A}_{j}}\right]_{\tilde{A}_{\text {classic }}} .
$$

In practice, a maximal Matsubara frequency $n_{\max }$ compatible with the error rescaling merit is determined, and all data $\operatorname{Im} G\left(\mathrm{i} \varphi_{m}, \mathrm{i} \omega_{n}\right)$ in $T^{C_{\varepsilon}}$, with $n \leq n_{\max }$ are used for inference. Presumably, better data selection strategies do exist. For example, using independent measurements for $\operatorname{Re} G$ and taking them into account by using a Schwarz representation (see equation (5.39)) could yield better results. Furthermore, the largest Matsubara frequency index $n_{\max }$ could be determined for each $\varphi_{m}$ individually. The latter appears to be necessary for non-equilibrium data.

For the truncation of singular values, a threshold $\lambda$ is used in Bryan's algorithm,

$$
\sigma_{i} \mapsto \begin{cases}\sigma_{i}, & \text { if } \sigma_{i} \geq \lambda \sigma_{1} \max \{M, N\}, \\ 0, & \text { else }\end{cases}
$$

for an $M$ by $N$ kernel matrix. While for the conventional Wick rotation $\lambda \approx 10^{-8}$ is sufficient, $\lambda \approx 10^{-12}$ has to be chosen in our case in order to take all relevant search directions in the $\tilde{A}$ space into account. Quadruple precision floating point arithmetic is found to be unnecessary. For discretizing the $\tilde{A}(\underline{x})$ function, logarithmic meshes for the $x_{1}$ and $x_{2}$ variables are used. Although $\tilde{A}(\underline{x})$ does not decay for all directions as $\underline{x} \rightarrow \infty$, choosing a finite mesh and truncating the integrals is not found to be critical.

The entropy expression for $\tilde{A}$ with respect to the default model $\tilde{D}$ is given by

$$
S[\tilde{A}]=\iint \mathrm{d}^{2} x\left(\tilde{A}(\underline{x})-\tilde{D}(\underline{x})-\tilde{A}(\underline{x}) \log \frac{\tilde{A}(\underline{x})}{\tilde{D}(\underline{x})}\right) .
$$




\section{Equilibrium limit}

Despite its naïvety, the equilibrium limit of the single-wedge approach yields decent results. As a test, we can analytically continue the $\varphi_{m}=0$ data via the MaxEnt approach to the conventional Wick rotation

$$
G\left(\mathrm{i} \varphi_{m}=0 ; \mathrm{i} \omega_{n}\right)=\int \frac{A(\varepsilon)}{\omega_{n}-\varepsilon} \mathrm{d} \varepsilon
$$

Figure 6.5 compares this $1 \mathrm{D}$ spectral function to the result based on the $2 \mathrm{D}$ data set $G\left(\mathrm{i} \varphi_{m}, \mathrm{i} \omega_{n}\right)$ and continued using the domain $T^{C_{\varepsilon}}$ and Vladimirov's Poisson kernel (5.45). As default models for high temperatures we introduce Lorentzian-shaped functions with variable width $\Gamma_{\text {default }}$. They read

$$
D(\omega)=\frac{1}{\pi} \frac{\Gamma_{\text {default }}}{\omega^{2}+\Gamma_{\text {default }}^{2}}
$$

for the 1D continuation and

$$
\tilde{D}(x, \omega)=\frac{1}{\pi} \frac{\tilde{\Gamma}_{\text {default }}(x)}{\omega^{2}+\tilde{\Gamma}_{\text {default }}(x)^{2}}
$$

for the $2 \mathrm{D}$ continuation, with $\tilde{\Gamma}_{\text {default }}(x)=\sqrt{\Gamma_{\text {default }}^{2}+x^{2}}$. An annealing procedure in the temperature is used for both, the $1 \mathrm{D}$ and $2 \mathrm{D}$ data for invoking adequate prior information, i.e. we use the $\tilde{A}$ solution of the next higher temperature as default model, starting with the Lorentzian at the highest temperature.

This annealing-type default model selection procedure appears not to have any strict Bayesian justification, however the physical argument is freezing out the highfrequency degrees of freedom and using present data for inferring low-energy details of the spectrum step by step [35]. Note that Gaussian default models are not well-suited for our data, since the high-frequency tail in the wide-band limit is Lorentzian. This manifests itself quantitively in the following way: For the Gaussian default models we tested all had $\operatorname{Pr}(\tilde{D} \mid \bar{G})$ one order of magnitude lower than the Lorentzian ones. For both, the Gaussian and the Lorentzian, we can expect the quantity $\Gamma_{\text {default }} / \Gamma$ to be $>1$, due to the overall broadening introduced by a finite interaction $U$.

Indeed, for the parameters $U=5 \Gamma, V_{G}=0, \Phi=0$ shown in Fig. 6.5(d), the (unnormalized) posterior probabilities $\operatorname{Pr}(D \mid \bar{G})$ and $\operatorname{Pr}(\tilde{D} \mid \bar{G})$ as a function of the parameter $\Gamma_{\text {default }}$ are peaked at $\approx 2 \Gamma$ for both, the $1 \mathrm{D}$ and $2 \mathrm{D}$ continuation procedures, respectively. These probabilities are calculated for $\beta=10 \Gamma^{-1}$. The most probable $\Gamma_{\text {default }}$ is chosen as default model. However, a strong dependence of the results on $\Gamma_{\text {eff }}$ is not observed.

The spectral functions shown in Fig. 6.5 are obtained for $\beta \Gamma=5,10$, and 20 . The sufficiently small value $\varepsilon=\frac{2}{19}$ had to be chosen for the $2 \mathrm{D}$ domain. The maximum 


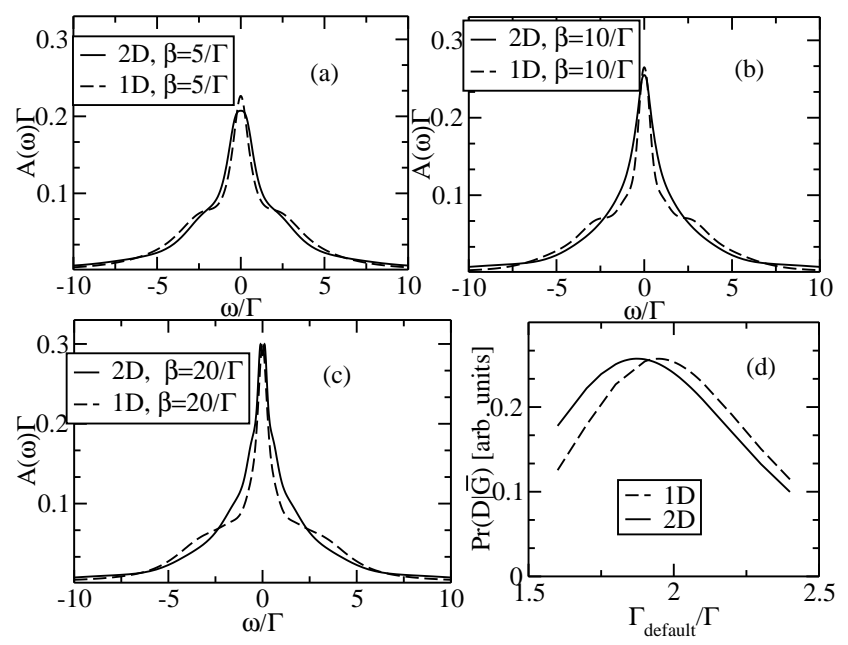

Figure 6.5: Analytically continued data for equilibrium $(\Phi=0)$ obtained using the conventional Wick rotation (1D) and the unconventional two-variable continuation (2D), with $U=5 \Gamma$, with particle-hole symmetry. The domain parameter for $2 \mathrm{D}$ continuation is $\varepsilon=\frac{2}{19}$. Subfigure (d) shows the posterior probabilities $\operatorname{Pr}(D \mid \bar{G})$ of the default models as a function of $\Gamma_{\text {default }}$ (Eqs. (6.27) and (6.28)).

number of Matsubara frequencies were $n_{\max }=10,20,40$ for $\beta \Gamma=5,10$, and 20, respectively. Note that due to the simple data selection strategy described in the previous section only data points with $\varphi_{-2} \leq \varphi_{m} \leq \varphi_{2}$ were taken into account. Using a global $n_{\max }$, the finite-sample estimate for the covariance submatrix $C^{(m=0)}$ in Eq. (6.9) eventually becomes singular, even though $C^{(m)}$ with $|m| \geq 3$ and $\omega_{n}>$ $\omega_{n_{\max }}$ could still be estimated for a limited set of Matsubara frequencies. Using such additional, well-estimated $C^{(m)}$ might lead to more structured spectral functions. In practice, however, the merit $\sigma$ must yet be viewed as a rather crude measure of the quality of the covariance estimate. So for the purpose of both simplicity and reproducibility we used the stronger restriction.

The Kondo temperature for $U=5 \Gamma$ is $T_{\mathrm{K}} / \Gamma \approx 0.1$, i.e. we can expect first signatures of strong coupling physics like Hubbard bands and a temperature dependent quasi-particle peak of reduced width in the spectra. Indeed both the $1 \mathrm{D}$ and $2 \mathrm{D}$ MaxEnt reproduce these features. More importantly, the overall shape of the spectra obtained agrees for all temperatures shown in Fig. 6.5(a-c). The results depend only slightly on the choice of $\Gamma_{\text {default }}$ for relevant values of $\operatorname{Pr}(\tilde{D} \mid \bar{G})$. Although the spectra inferred from the $2 \mathrm{D}$ procedure using our current implementation appear to be less structured, the overall shape seems to be reconstructed quite well. For more serious calculations, the detailed high-frequency behavior (and behavior for large $\underline{x}$ ) can be introduced with a more sophisticated default model, e.g. based on perturbation theory. 


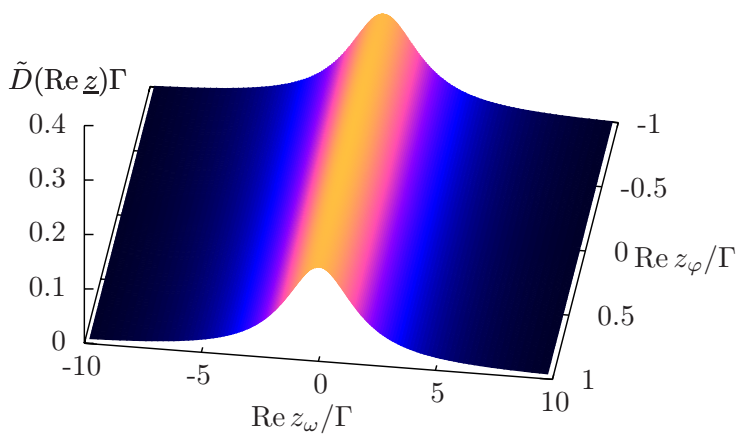

(a) default model

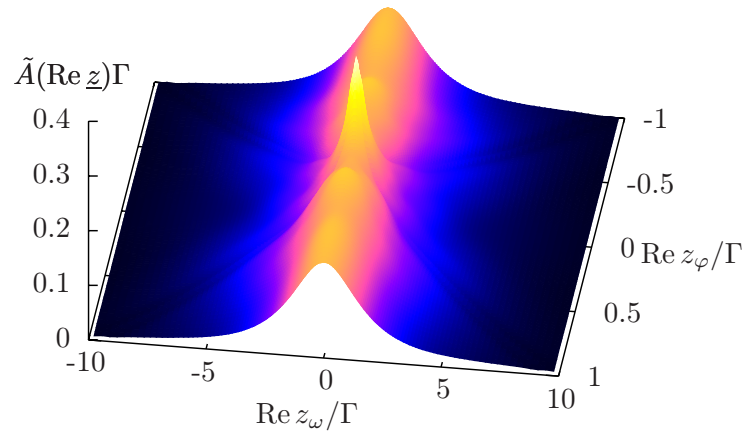

(b) MaxEnt solution

Figure 6.6: Lorentzian default model (6.28) with best $\operatorname{Pr}(\tilde{D} \mid \bar{G})$ for first annealing step in equilibrium, and MaxEnt solution for $\tilde{A}\left(x_{\varphi}, x_{\omega}\right)$ inferred from the QMC data at the lowest temperature, $\beta \Gamma=20$, for the equilibrium test case shown in Fig. 6.5.

\section{Inferred Edge function $\tilde{A}\left(x_{\varphi}, x_{\omega}\right)$}

Figure 6.6a shows the Lorentzian default model we used at the highest temperature in the annealing procedure, $\beta \Gamma=2$. At the lowest temperature $\beta \Gamma=20$, the representation shown in Fig. $6.6 \mathrm{~b}$ was obtained. The equilibrium spectral function shown in Fig. $6.5(\mathrm{c})$ is given by the cut $\tilde{A}(\Phi=0, \omega)$. Other values of $\operatorname{Re} z_{\varphi}$ do not have any physical meaning.

Note that certain structures appear in the inferred $\tilde{A}\left(x_{\varphi}, x_{\omega}\right)$ which vary as the domain parameter $\varepsilon$ is changed: they occur for $\operatorname{Re} z_{\varphi}= \pm \operatorname{Re} \varepsilon z_{\omega}$. They can be interpreted as resulting from the fundamental properties of the kernel function discussed in Sec. 6.2.2 in combination with the MaxEnt principle of only incorporating changes which are strongly supported by data. Also, at larger distance from the origin, discretization errors from the discretization of the double integral are most dominant for this most structured region of the kernel. At finite bias, the qualitative structure of the inferred representation remains unchanged.

\section{Finite bias voltage}

The rule of thumb $n_{\max } \approx \frac{\beta U}{2}$ appeared to be a good choice for preparing the equilibrium QMC data for inference. For the nonequilibrium $\Phi>0$ a first interesting observation is that at sufficiently low temperatures $n_{\max }$ seems to be considerably smaller than $\frac{\beta U}{2}$.

In fact, at least the simple data selection strategy yielding $n_{\max }$ does not appear to produce a sufficiently informative data set to obtain quantitative agreement with for example real-time QMC calculations [21]. This severe problem was observed for $\beta \Gamma=10$ and the interaction strengths $U=4 \Gamma$ and $U=6 \Gamma$ and several values of the bias voltage $\Phi$. As a result, the MaxEnt leaves the nonequilibrium spectral function 
invariantly equal to the default model's distribution. The reason for this behaviour is due to the geometry of the continuation problem, as explained in section 6.2.2.

Nevertheless, as shown in appendix A, the single-wedge continuation problem in principle allows for unique solutions. It is thus interesting to see to which extent the MaxEnt can be used within this simple setup in order to compute nonequilibrium spectra and conductance data. By picking an $n_{\max }$ for each $\varphi_{m}$ separately, driving the applicability of the MaxEnt to a limit, larger sets of admissible input data were obtained. At least for small voltages, they eventually produce MaxEnt changes in the nonequilibrium spectral function based on the data, yielding currents which show show a better agreement with real-time data for the current-voltage characteristics.

Because the procedure is rather arbitrary and at the edge of the applicability of the MaxEnt, the following criteria were used to restrict the choices of data sets producing convergent MaxEnt solutions:

- ensure an error rescaling $\sigma \approx 1$;

- discard strongly oscillating solutions and solutions with obvious artifacts around $\omega \approx 0$

- discard solutions which strongly violate the physical sum rule $\|A\|:=\int \mathrm{d} \omega A(\omega)=$ 1. In many cases, too small values $\|A\| \approx 0.9$ were obtained. Note that the MaxEnt as we implemented it only has prior information about the value of the truncated double integral $\iint \mathrm{d}^{2} x \tilde{A}(\underline{x})$, because two-dimensional probability densities are considered when the entropy expression (6.25) is straightforwardly generalized with respect to $\tilde{A}$;

- use as many data points as possible, starting with small $\omega_{n}$, to maximize the amount of accessible information.

Note that the domain parameter $\varepsilon$ was, again, chosen somewhat arbitrarily: For $U=4 \Gamma$ we only investigated $\nu_{\max }=16$, for $U=6 \Gamma$ we picked $\nu_{\max }=20$, with $\varepsilon=\frac{2}{\nu_{\max }-1}$. The dependence of the results on the particular choice of $\varepsilon$ was not studied systematically yet, but work along these lines is under way and the results will be presented elsewhere. The usual annealing procedure with temperatures $\beta \Gamma=2$, $\beta \Gamma=5, \beta \Gamma=10$, where for $\beta \Gamma=2$ the Lorentzian default models with $\Gamma_{\text {default }}=1.5 \Gamma$ $(U=4 \Gamma)$ and $\Gamma_{\text {default }}=2.1 \Gamma(U=6 \Gamma)$ were found to be most suitable based on the posterior $\operatorname{Pr}(\tilde{D} \mid \bar{G})$.

The source-drain current $J$ was computed using the Meir-Wingreen equation (6.19),

$$
J=J_{\max } \int \mathrm{d} \omega\left[f_{L}(\omega)-f_{R}(\omega)\right] A(\omega)
$$

with $J_{\max }=\frac{\Gamma e}{h}$. Again, it should be emphasized that due to the estimator bias from the default model, for too small sets of QMC data the method systematically underestimates the current, because Bryan's algorithm by convention does not incorporate 


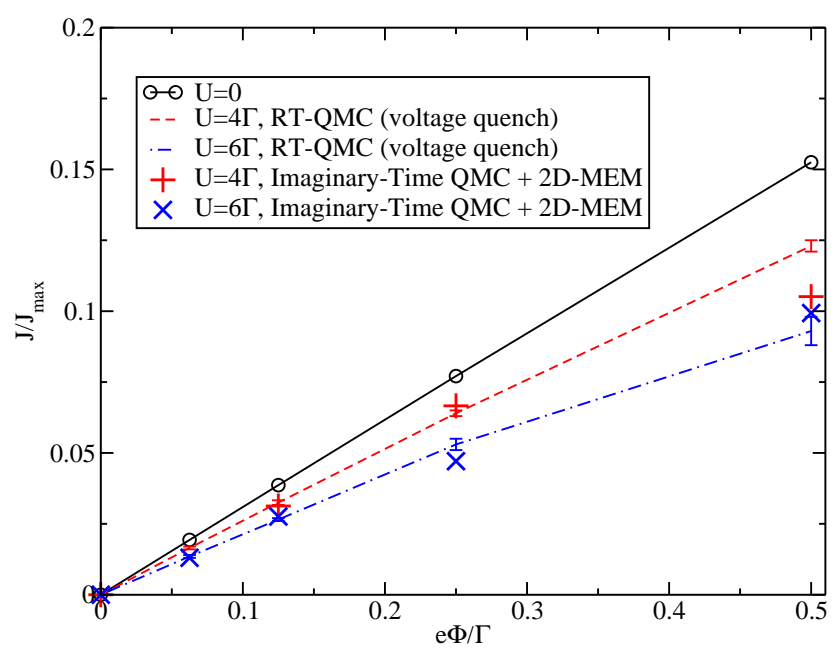

Figure 6.7: Current-voltage characteristics obtained using the single-wedge MaxEnt inference of Matsubara-voltage theory compared to RT-QMC [21] data for indicated Coulomb interactions at inverse temperature $\beta \Gamma=10$.

any changes to $\tilde{A} \approx \tilde{D}$ in case the data do not provide sufficient evidence for such modifications. As a result, the current is too small, because in the vicinity of $\omega \approx 0$ the less structured default model obtained from the next higher temperature (initially the broad Lorentzian (6.28)) is much flatter than the true solution, which features a sharp Abrikosov-Suhl resonance in the relevant frequency range. Hence, the spectral function obtained from the MaxEnt has less spectral weight in the integration window in Eq. (6.29) than the true $A(\omega)$. Due to this trend of underestimation, in Fig. 6.7 we compare the largest values of the current compatible with the above-listed restrictions to data obtained using a recently developed real-time approach [21]. A generally good agreement is obtained. However, the data selection procedure is too arbitrary to consider these results unbiased. Error bars are not available. If we only considered a fixed set of data $\bar{G}$, the covariance $\operatorname{Cov}\left(\tilde{A}\left(\underline{x}^{(1)}\right), \tilde{A}\left(\underline{x}^{(2)}\right)\right)$ would be estimated easily [118]. However, due to large off-diagonal terms, attempting to estimate an error bar for $J$ is rather cumbersome. The $e \Phi / \Gamma=0.0625$ run did not converge to a solution meeting our criteria for $U=4 \Gamma$ and was not taken into account.

\section{Non-Equilibrium Spectral Functions}

Spectra resulting from the procedure described above are shown in Fig. 6.8. These are the spectral functions used to compute the current in Fig. 6.7.

Because the data selection criteria for the nonequilibrium case drive the input data range for the MaxEnt to a limit, neglect the error of the associated covariance estimate [121], and - maybe most importantly - rely on a somewhat arbitrarily chosen $\gamma_{\text {crit }}$, an overfitting resulting in oscillations in the spectra is obtained. For these reasons, 


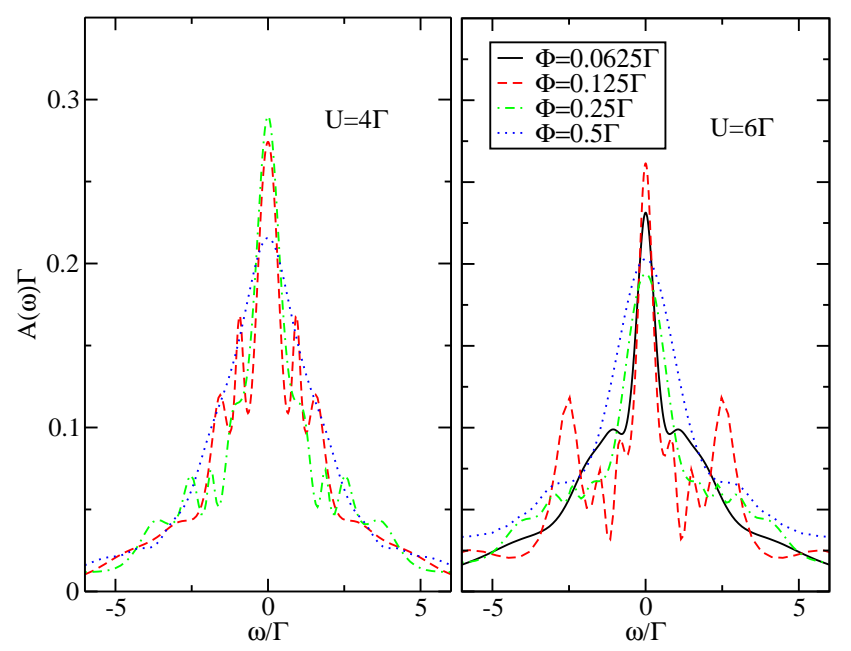

Figure 6.8: Spectra $A(\omega)=\tilde{A}(\Phi, \omega)$ used for the computation of the current shown in Fig. 6.7.

the oscillations are pure numerical artifacts and cannot be traced back to a formal inconsistency of the theory beyond the neglected influence of branch cuts within $T^{C_{\varepsilon}}$, because the inferred equilibrium functions, Fig. 6.5 and Fig. 6.6b, do not show these features although they result from the same formal analytical structure. In fact, when artificial noise is added to the free Green's function, a strong overfitting is obtained whenever a wrong covariance estimate is passed to the MaxEnt. Therefore, the continuation problem is merely more demanding from a stochastic point of view as discussed in section 6.2.2.

A tendency towards suppression of spectral weight is observed at small $\omega$ with increasing $U$. This is consistent with the expectation that the quasi-particle resonance for $U=6 \Gamma$ is already reduced, because $\beta^{-1}=0.1 \Gamma>T_{K}$, whereas $\beta^{-1} \approx T_{K}$ for $U=4 \Gamma$.

In Fig. 6.9, we show a comparison of the spectral functions for $U / \Gamma=4$ and $\beta \Gamma=10$ to the result obtained from fourth-order perturbation theory [122]. Based on the results presented in Ref. [21], we expect that fourth-order perturbation theory is quite accurate at this interaction strength and temperature. Besides the unphysical oscillations in the MaxEnt result and a bias towards the high-temperature default model, especially for larger voltage biases, the agreement between the spectral functions seems satisfactory.

Table 6.1 shows the norm $\|A\|=\int A(\omega) \mathrm{d} \omega$ for the functions presented in the figure. Obviously, the physical sum rule $\|A\|=1$ is not strictly obeyed, and there is a slight tendency towards too small norms whose origin is unclear but which appears to be consistent with the trend of current underestimation. Moreover, the selection of data we chose at $\beta \Gamma=10$ for $U=4 \Gamma$ and $U=6 \Gamma$ is shown in table 6.1 and table 6.3 , 


\begin{tabular}{|c||c|c|}
$\Phi / \Gamma$ & $\|A\|_{U=4 \Gamma}$ & $\|A\|_{U=6 \Gamma}$ \\
\hline 0.0625 & - & 0.91 \\
0.125 & 0.92 & 0.92 \\
0.25 & 0.92 & 0.95 \\
0.5 & 1.03 & 1.16
\end{tabular}

Table 6.1: Norms of the spectral functions shown in Fig. 6.8.

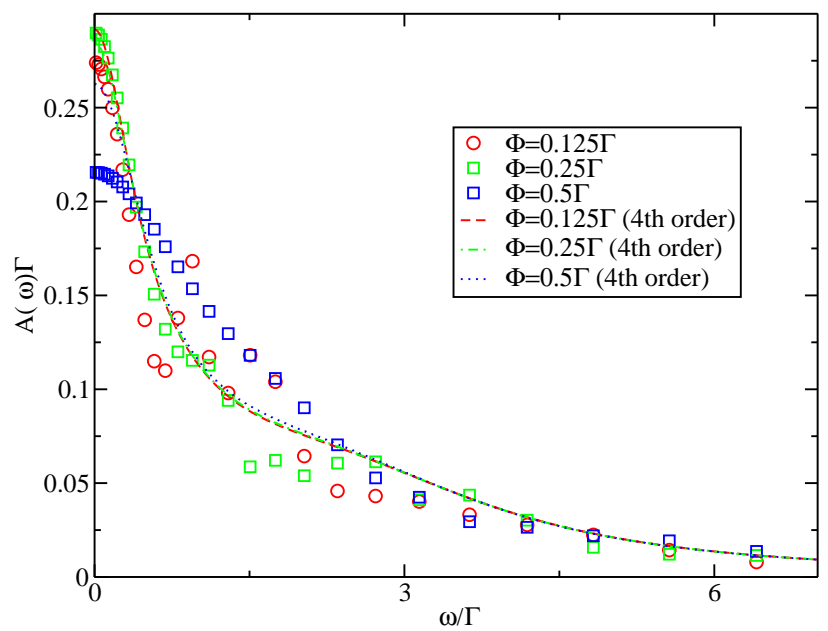

Figure 6.9: Spectra $A(\omega)=\tilde{A}(\Phi, \omega)$ for $U=4 \Gamma$ as compared to fourth-order perturbation theory.

respectively. The tables present the number $N_{m} \approx n_{\max }(m)-2 m / \varepsilon$ of Matsubara frequencies which are located within the cone domain $T^{C_{\varepsilon}}$ for the chosen $n_{\max }(m)$. Larger values of $m$ were not considered, although at least $m= \pm 4$ yields further relevant information about $\tilde{A}$. For a test case the spectra did not show dramatic qualitative changes as additional values at larger $\varphi_{m}$ were included, as long as the error scaling merit remained $\sigma \approx 1$. However, the level of arbitrariness in the data selection would have been even larger, because of the corresponding additional $n_{\max }$ parameters.

Apart from these in principle correct features, obtaining reliable nonequilibrium spectral functions will obviously require more effort. In principle, apart from the recently discovered existence of the $\operatorname{Im} z_{\varphi}=0$ branch cut, better nonequilibrium spectral functions should be obtained by using longer QMC runs, as long as the choice of the critical branch cut index $\gamma_{\text {crit }}$ is adequate. However, increasing the number of Monte-Carlo sweeps, we found that in some cases the noise on the spectral function increased as the QMC data accuracy was improved, while in other cases the quality increased. The fact, that the quality of the output spectra does not 


\begin{tabular}{|c||c|c|c|c|}
$\Phi / \Gamma$ & $N_{m=0}$ & $N_{m= \pm 1}$ & $N_{m= \pm 2}$ & $N_{m= \pm 3}$ \\
\hline 0.0625 & - & - & - & - \\
0.125 & 26 & 12 & 6 & 3 \\
0.25 & 24 & 12 & 5 & 3 \\
0.5 & 24 & 12 & 6 & 3
\end{tabular}

Table 6.2: Number $N_{m}$ of Matsubara frequencies taken into account for each value of $m$ taken into account in the data selection at $\beta \Gamma=10$ for $U / \Gamma=4$ and for the voltages plotted in Fig. 6.7.

\begin{tabular}{|c||c|c|c|c|}
$\Phi / \Gamma$ & $N_{m=0}$ & $N_{m= \pm 1}$ & $N_{m= \pm 2}$ & $N_{m= \pm 3}$ \\
\hline 0.0625 & 20 & 11 & 6 & 1 \\
0.125 & 21 & 11 & 6 & 8 \\
0.25 & 21 & 11 & 6 & 3 \\
0.5 & 20 & 11 & 6 & 1
\end{tabular}

Table 6.3: Same as Table 6.1 but for $U / \Gamma=6$.

improve systematically with the data quality illustrates the inherent difficulty of the approach. Apparently, in cases, where the noise grows as function of the number of QMC sweeps, the neglected branch cuts within the $T^{C_{\varepsilon}}$ domain become important. In particular, this is expectable for the $\operatorname{Im} z_{\varphi}=0$ branch cut. This problem appears to be amplified by the increased difficulty of the continuation problem at finite bias (see section 6.2.2). An elimination of the restriction to the domain $T^{C_{\varepsilon}}$ and thus the necessity to arbitrarily determine a critical branch cut index is therefore highly desirable. Chapter 7 goes along this line and introduces a formal elimination of the function-theoretical restriction of the data space to the cone domain $T^{C_{\varepsilon}}$.

\section{Optional Extension: Taking into account the $\operatorname{Im} z_{\varphi}=0$ branch cut}

In order to take into account the $\operatorname{Im} z_{\varphi}=0$ branch cut explicitly within the singlewedge continuation strategy, one could use the $\left(\mathrm{i} \varphi_{m}-\Phi\right) \leftrightarrow-\left(\mathrm{i} \varphi_{m}-\Phi\right)$ invariance at symmetric coupling to the leads (see section 3.4.4) and - in terms of figure 6.4 - cut $T^{C_{\varepsilon}}$ into an upper and lower part. The functions living on $T^{C_{\varepsilon}}$ can then be parametrized via the edge of the upper part, using the symmetry relation.

An unanswered question about this approach is how to appropriately incorporate the information about low-energy physics from the $i \varphi_{m}=0$ data, which are located on the boundary of upper and lower half of $T^{C_{\varepsilon}}$. Apart from this, the numerical performance of this refined strategy has not been tested within this work, because it is also expected to be limited by the following aspects in principle. 


\subsubsection{Geometry of the Single-Wedge Inference Problem}

Very important insights result from the structure of the kernel function (5.45) and its role in the problem to reconstruct function values on the edge from data within a given wedge. In the language of Bayesian inference, the kernel function defines the information channel through which evidence about the shape of the representation function $\tilde{A}(\underline{x})$ and thus also the physical spectral function $A(\omega)$ is extracted from the Monte Carlo data.

For the information provided by a single data point, this channel may result in rather vague or rather strong evidence for changes in a given compact region $R \subset V_{\tilde{A}}$, see Eq. (6.6). It depends on whether the subset of column vectors $u_{i}$ of $U$ spanning $R$ is associated with rather small or rather large singular values $\sigma_{i}$, respectively. Furthermore, the overlap of the column vectors $v_{i}$ of $V$ with the data point is important. For example, in the one-dimensional continuation problem (6.1) one is most reliably able to extract low-energy features (see below).

For this reason, very small singular values yield irrelevant components of the channel and are therefore projected out in Bryan's algorithm by introducing the threshold $\lambda$, Eq. (6.24). We can neither perform the SVD analytically, nor can we analytically take into account structural changes which occur when rotating the basis of $V_{\text {data }}$ to the eigenbasis of the covariance matrix $C$ in order to consider statistically independent data. We can however consider values of the kernel in $V_{\tilde{A}}$ for a given data point, assuming it to be uncorrelated with other data points so that it may be investigated separately. Within our QMC implementation, the structure of measured covariance matrices reveals that correlations between Matsubara frequencies $\omega_{n}, \omega_{n^{\prime}}$ monotonically decrease as a function of distance $\left|\omega_{n^{\prime}}-\omega_{n}\right|$, though very slowly.

Let us first consider a single uncorrelated imaginary part of a Green's function at Matsubara frequency $\omega_{n}$ in the standard Wick rotation problem. The spectral function $A(\varepsilon)$ is inferred through the Lorentzian-shaped kernel (6.1),

$$
\operatorname{Im} K_{\mathrm{eq}}[A(\varepsilon)]\left(\omega_{n}\right)=-\frac{\omega_{n}}{\varepsilon^{2}+\omega_{n}^{2}} .
$$

For all $\omega_{n}$, the kernel (6.1) is centered around $\varepsilon=0$ and higher frequencies are associated with larger values of the kernel as the width given by $\omega_{n}$ is increased. As compared to $\varepsilon \approx 0$ the values of the kernel at large frequencies are still small. We can therefore expect large singular values and thus relevant components of the kernel to be associated with small frequencies only. This is in agreement with the well-known observation [35] that high-frequency information about the spectral function is better put into the default model as prior knowledge and a good resolution is obtained for the - fortunately most interesting - low-frequency region.

In the case of our two-dimensional continuation, the situation is quite similar. For 
given data $\operatorname{Im} G\left(\mathrm{i} \varphi_{m}, \mathrm{i} \omega_{n}\right)$, the Poisson kernel, Eq. (5.45), is

$$
\mathcal{P}_{C_{\varepsilon, 0}}\left(\left(\mathrm{i} \varphi_{m}-x_{\varphi}, \mathrm{i} \omega_{n}-x_{\omega}\right)^{T}\right)=\frac{1}{\pi^{2} \varepsilon} \prod_{\mu= \pm 1} \frac{\omega_{n}-\mu \varphi_{m} / \varepsilon}{\left(x_{\omega}-\mu x_{\varphi} / \varepsilon\right)^{2}+\left(\omega_{n}-\mu \varphi_{m} / \varepsilon\right)^{2}}
$$

It is the product of two Lorentzians.

In analogy to the argument given above, one may expect the best resolution for data $\tilde{A}\left(\underline{x}^{(\text {best })}\right)$ with

$$
x_{\omega}^{(\text {best })} \approx \pm x_{\varphi}^{(\text {best })} / \varepsilon \text { and } x_{\omega}^{(\text {best })}, x_{\varphi}^{(\text {best })} \approx 0 .
$$

This is illustrated in figure 6.10 for some assorted values of $i \varphi_{m}$ and $i \omega_{n}$. One can see that the orientation of the imaginary-time data point defines the orientation of the best-resolved area on the edge. As a consequence, in contrast to equilibrium, the low-energy region $\left(x_{\omega} \approx 0\right)$ of the spectral function is not always the best resolved one.

In other word, for a fixed opening ratio $\varepsilon$, the structure of the best-resolved region (6.31) does not depend on the physical voltage $\Phi$. In the limit of very large voltages, especially the low-frequency region of the physical spectrum $A(\omega)=\tilde{A}(\Phi, \omega)$ is not expected to be in the best resolvable region (6.31).

Thus, the approach based on a representation of data in $T^{C_{\varepsilon}}$ appears to be limited to relatively small voltages. On top of this, the low-frequency region of the nonequilibrium spectral function is, even for small voltages, not contained in the well-inferred region of $\tilde{A}$. It should be evident from figure 6.10 why the sharp cross-like structure in figure $6.6 \mathrm{~b}$ arises and that it corresponds to the angle-dependent geometry of the inverse problem.

\section{General Wedges}

Due to the biholomorphic equivalence of all $T^{C_{r, \vartheta}}$ wedges (section 5.4.2), an analogue geometry of the inverse problem arises for inference of edge values of functions living on the rotated wedges, $\vartheta \neq 0$. It is displayed in figure 6.11. As can be seen, the low-energy region of the cut which was corresponding to the nonequilibrium spectral function is now well-resolved. Unfortunately, the upper edge function corresponds to the wrong limiting procedure, and not the physical (5.11). The key task thus appears to be providing a link between the corresponding regions of the edge functions in $\left(x_{\varphi}, x_{\omega}\right)$-space, in order to improve the resolution of the nonequilibrium spectral function. Possible approaches will be discussed and developed in chapter 7 . 


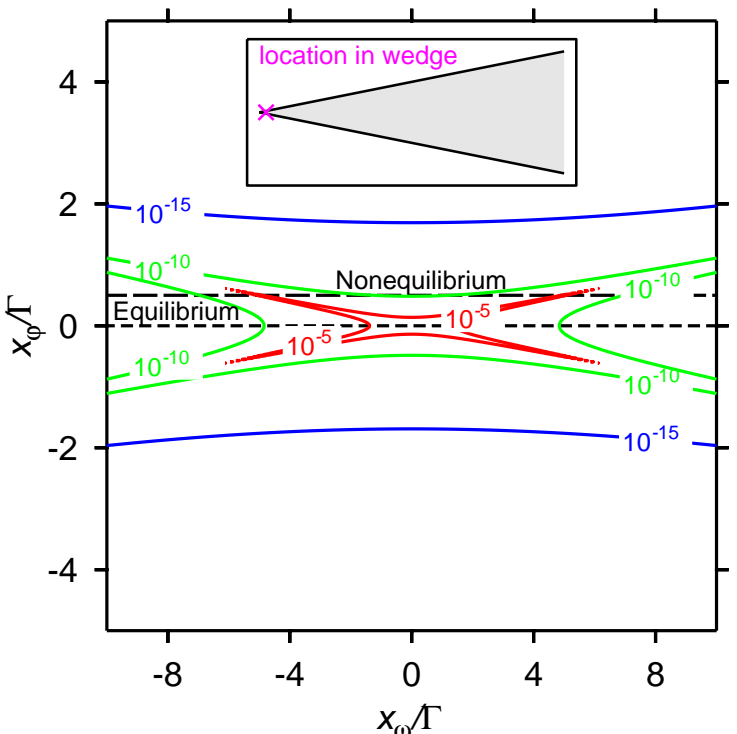

(a) $m=0, n=0$

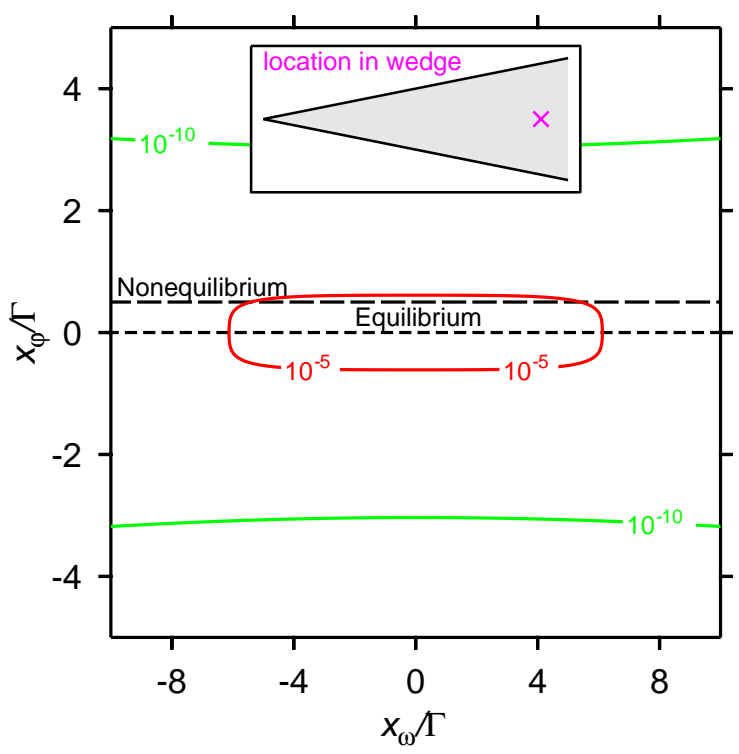

(c) $m=0, n=20$

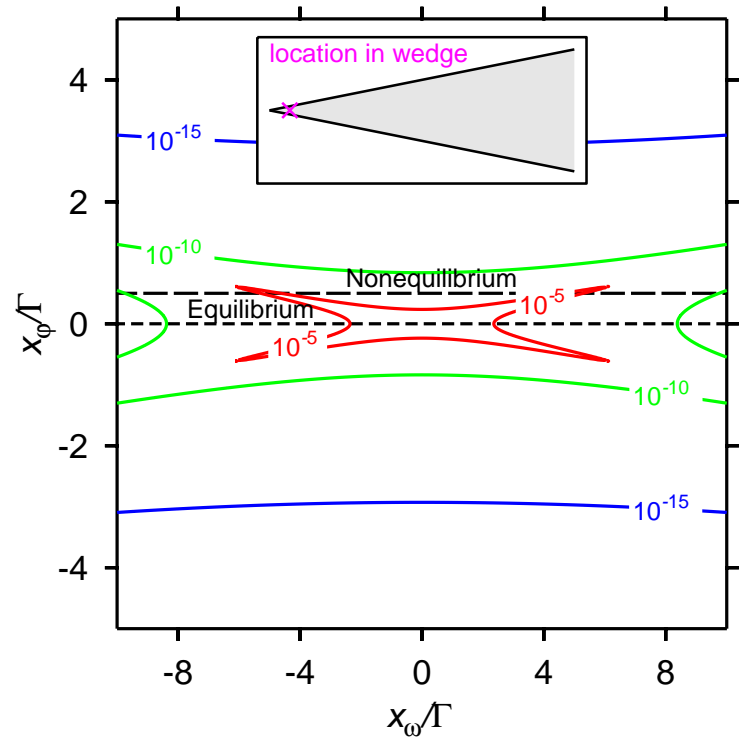

(b) $m=0, n=1$

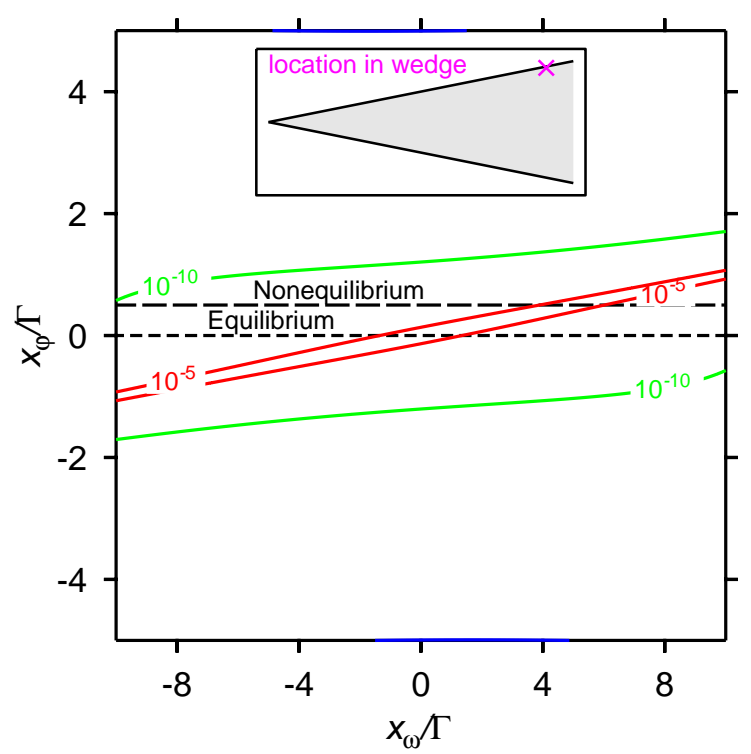

(d) $m=1, n=20$

Figure 6.10: Geometry of the single-wedge inverse problem. A contour plot of $\mathcal{P}_{C_{\varepsilon, 0}}\left(\left(\mathrm{i} \varphi_{m}-\right.\right.$ $\left.\left.x_{\varphi}, \mathrm{i} \omega_{n}-x_{\omega}\right)^{T}\right)$ vs. $\left(x_{\varphi}, x_{\omega}\right)^{T}$ is shown for some chosen combinations of $\mathrm{i} \varphi_{m}$ and $\mathrm{i} \omega_{n}$ at a wedge opening ratio $\varepsilon=0.1$. Inverse temperature is $\beta=20.0$. In case of the equilibrium theory $(\Phi=0)$, the spectral function is located on the short-dashed line. In the nonequilibrium case $(e \Phi / \Gamma=0.5)$, the spectral function is on the long-dashed line. 


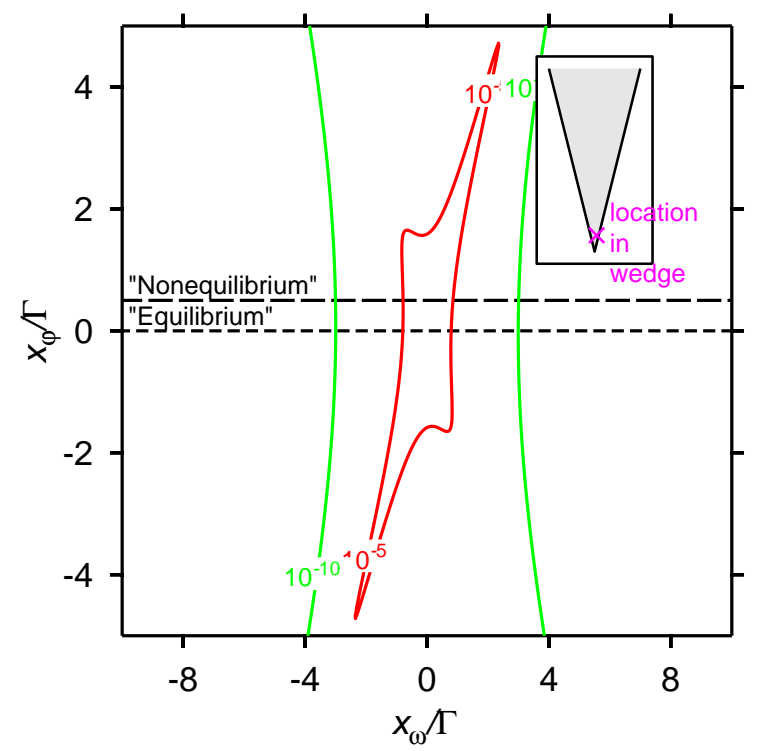

(a) $m=1, n=0$

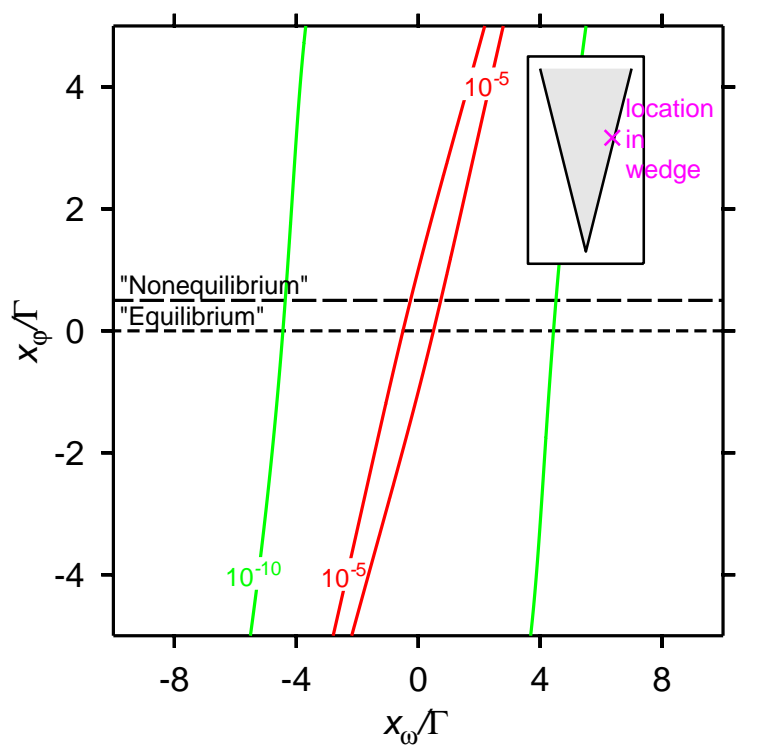

(b) $m=7, n=6$

Figure 6.11: Same as Fig. 6.10, but for the connection of upper wedge and the upper edge function, i.e. $\mathcal{P}_{C_{r=1 / 2, \vartheta=\pi / 2}}\left(\left(\mathrm{i} \varphi_{m}-x_{\varphi}, \mathrm{i} \omega_{n}-x_{\omega}\right)^{T}\right)$. The upper edge function is not directly related to the physical spectrum. But if there is a simple relation between edge functions which is local in $\underline{x}$, the resolution of nonequilibrium spectra is improved dramatically.

\subsection{Summary}

The MaxEnt technique for the analytic continuation of quantum Monte-Carlo data was introduced. We showed that for static observables, namely the double occupancy and the magnetic susceptibility, the Matsubara-voltage approach yields physically consistent expectation values. The magnetic susceptibility was found to be a sensitive probe of the Kondo effect out of equilibrium which is in reasonable quantitative agreement with the SNRG. Discrepancies in the double occupancy as obtained from different numerical methods were pointed out.

Based on the mathematical preliminaries of the previous chapter, we set up a MaxEnt method for the two-variable analytic continuation of the Green's function. The method successfully reproduced equilibrium spectral functions but failed to yield reliable non-equilibrium spectral functions. The issue was mainly traced back to the kernel structure which results from considering a single sharp wedge containing only a subset of the raw data. In order to overcome this problem, the next chapter will develop a method to systematically incorporate data from different branches of the Green's function into the inference of the non-equilibrium spectral function. 


\section{Generalized Approach to the Double Analytic Continuation}

The aim of the present chapter is to extend the function-theoretical approach to spectral functions of section 6.2 to a computationally feasible method for true nonequilibrium properties. To do so, a relation between the wedges has to be found, in order to use data of all wedges for the determination of the nonequilibrium spectral function. We will introduce a connection based on a continuity property of $\operatorname{Re} G$ at the branch point of the wedges. ${ }^{1}$ From a-priori considerations, it will be shown that the high-energy structure of the Green's function is described correctly. Furthermore, the continuation approach by Han and Heary [27] is included as a special case. ${ }^{2}$ It turns out that the approximation is an appropriate tool for computing spectral functions and transport properties for intermediate many-body correlations. An exact generalization of the algorithm will be suggested. Results from this section will be published in Ref. [123].

\subsection{Bogolyubov's edge-of-the-wedge theorem}

From a formal point of view, it should be mentioned that a candidate of a link between functions on wedges is in principle provided by Bogolyubov's famous edgeof-the-wedge theorem which was first discovered in the context of axiomatic quantum field theory [99]. It considered the analytic continuation of Wightman functions in order to establish certain dispersion relations. From a mathematical point of view, it also introduced a generalization of the very notion of analytic continuation [99].

There are several versions of the theorem. A simple version which captures the essential idea may be found in the book by Hörmander on partial differential operators [124]. It roughly considers two functions $f^{ \pm}$which are holomorphic on the tube cones $T^{ \pm C}$, where $C$ is a convex open cone with vertex at zero. Consequently, the edges of $T^{C}$ and $T^{-C}$ are "infinitesimal neighbours". If the functions have the same boundary value distributions, $f^{+}\left(\underline{x}+\mathrm{i} 0^{C}\right)=f^{-}\left(\underline{x}-\mathrm{i} 0^{C}\right)=: f_{0}$, then $f_{0}$ is an analytic function. $f_{0}$ provides an analytic continuation of both, $f^{+}$and $f^{-}$.

In its more general formulations, the theorem actually demands the functions $f^{ \pm}$ to be holomorphic only locally at the edge and establishes certain facts about the

\footnotetext{
${ }^{1}$ The branch point is the point around which the edges of the wedges are arranged (figure 3.4).

${ }^{2}$ The generalized approach with Padé corrections (Ref. [53]) is, however, not included.
} 


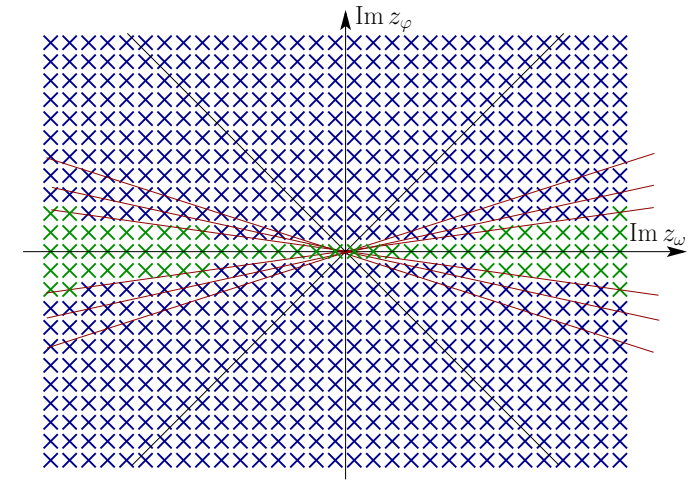

(a) imaginary-voltage formalism data

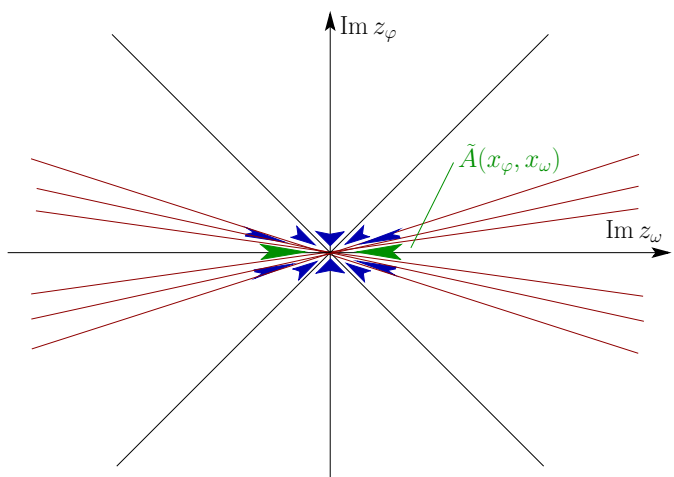

(b) corresponding set of Edge functions

Figure 7.1: Abstract picture of the analytic structure of dynamical correlation functions. The set of branch cuts has been reduced for reasons of clarity and comprehensibility. (a) and (b) are equivalent structures, due to the uniqueness theorem provided in appendix A. In particular, (b) is a unique decomposition of (a). They are linearly related by Vladimirov's representations such as equation (5.50). The green edge functions are MaxEnt-inferable due to the presumed positive/negative definiteness of the imaginary part. Due to their partial meaning as an advanced/retarded Green's function they also yield the spectral function. Only green data points in the left panel give direct evidence about physics (section 6.2.1).

domain in which $f_{0}$ is analytic (global edge-of-the-wedge theorem) [125].

Matching the geometry of our correlation functions, an extension to several cones whose edges meet in a single point is provided by Martineau's theorem [34]. As in Bogolyubov's theorem, locally, holomorphic functions may be found which constitute analytic continuations of pairs of functions living on wedges. Again, the edge values of the considered set of functions have to be interrelated in a more or less direct way.

\subsection{Systematic extension of the continuation procedure by use of edge relations}

In order to construct an explicit functional-analytic approach to the analytic continuation which would enable us to extend the numerical implementation of the maximum entropy approach, the edge-of-the-wedge theorem is only of indirect use. It is however clear that any simple relation between edges of the different branches of the Green's function provides a rather direct link between integral representations of the respective wedges. In particular, a simple identity among Edge functions would be of great help. This is illustrated in figure 7.1: an identity between the edge functions would make blue-colored data available for the inference of the physically relevant green-colored edge functions. At the same time, from a more technical point of view, 
it reduces the amount of fit parameters necessary to describe the entire analytic structure.

\subsubsection{Shared real part on edges}

A candidate for such a local relation between edge functions is the equality of all functions $\operatorname{Re} G_{0}\left(\underline{x}+\mathrm{i} 0^{\vartheta}\right)$ at arbitrary edge orientations of the bare Green's function, i.e. equation (5.57). It is easy to verify that same is true for the second-order self-energy (5.16). The continuation approach by Han and Heary in Ref. [27] also generates this identity. We have

$$
\begin{aligned}
\operatorname{Re} G_{0}\left(\underline{x}+\mathrm{i} 0^{\vartheta}\right) & =\operatorname{Re} G_{0}\left(\underline{x}+\mathrm{i} 0^{\vartheta^{\prime}}\right), \\
\operatorname{Re} \Sigma^{(2)}\left(\underline{x}+\mathrm{i} 0^{\vartheta}\right) & =\operatorname{Re} \Sigma^{(2)}\left(\underline{x}+\mathrm{i} 0^{\vartheta^{\prime}}\right),
\end{aligned}
$$

for all $\vartheta, \vartheta^{\prime} \in[0,2 \pi)$.

This structure is similar to the conventional Green's function causality relation

$$
G\left(z^{*}\right)=G(z)^{*}
$$

There, we consequently have

$$
\operatorname{Re} G\left(\omega+\mathrm{i} 0^{+}\right)=\operatorname{Re} G\left(\omega-\mathrm{i} 0^{+}\right) .
$$

However, in our case we only know for sure the symmetry (3.96),

$$
G\left(z_{\varphi}^{*}, z_{\omega}^{*}\right)=G\left(z_{\varphi}, z_{\omega}\right)^{*}
$$

From equation (7.5) only the edge relation

$$
\operatorname{Re} G\left(\underline{x}+\mathrm{i} 0^{\vartheta+\pi}\right)=\operatorname{Re} G\left(\underline{x}+\mathrm{i} 0^{\vartheta}\right), \vartheta \in[0, \pi)
$$

can be derived. I.e., conjugate wedges $T^{C}, T^{-C}$ carry the same real parts of $G(\underline{z})$ on their edges.

\subsubsection{A-priori constraints to the shared-real-part assumption}

We will now investigate to which extent the continuity relations (7.1) and (7.2) can a priori be expected to hold for higher-order contributions to the fully interacting Green's function $G(\underline{z})$. It is insightful to study the algebraic properties of a conventional Green's function $G(z)$ first and subsequently consider the two-variable function $G(\underline{z})$. 


\section{Conventional Green's function}

As a simple example, let us consider the summation of the Dyson series [97]

$$
\begin{aligned}
G & =G_{0}-G_{0} \Sigma G \\
& =G_{0}-G_{0} \Sigma G_{0}+G_{0} \Sigma G_{0} \Sigma G_{0}-\cdots .
\end{aligned}
$$

The entities $G(z), G_{0}(z)$, and $\Sigma(z)$ satisfy the causality relation (7.3). For the equation to hold, the product (and the sum) of two (7.3)-satisfying quantities $A(z), B(z)$ shall also satisfy (7.3). This is obviously the case, because

$$
\begin{aligned}
\operatorname{Re}(A B)\left(\omega+\mathrm{i} 0^{+}\right)= & \operatorname{Re} A\left(\omega+\mathrm{i} 0^{+}\right) \operatorname{Re} B\left(\omega+\mathrm{i} 0^{+}\right)-\operatorname{Im} A\left(\omega+\mathrm{i} 0^{+}\right) \operatorname{Im} B\left(\omega+\mathrm{i} 0^{+}\right) \\
= & \operatorname{Re} A\left(\omega-\mathrm{i} 0^{+}\right) \operatorname{Re} B\left(\omega-\mathrm{i} 0^{+}\right)- \\
& \left(-\operatorname{Im} A\left(\omega-\mathrm{i} 0^{+}\right)\right)\left(-\operatorname{Im} B\left(\omega-\mathrm{i} 0^{+}\right)\right) \\
= & \operatorname{Re}(A B)\left(\omega-\mathrm{i}^{+}\right) .
\end{aligned}
$$

The crucial point is that as one can see the conjugateness of the imaginary parts of the upper and lower functions is nearly mandatory for the equation to hold. Consequently, the set of functions with only the property (7.4) is not closed under multiplication.

\section{Two-variable Green's function}

Unfortunately, for a similar reason, the shared-real-part property (Eqs. (7.1), (7.2)) is not closed under multiplication. This can be checked explicitly with the terms $G_{0}(\underline{z})^{2}$ and $G_{0}(\underline{z})^{-1}$. Since also the two-variable function $G(\underline{z})$ is comprised of Dyson-series type of contributions (7.7), the shared-real-part assumption is only an approximation to the structure of the interacting Green's function. Fortunately, however, there are also some important a-priori constraints to the structure of the resulting error terms. They are summarized in appendix B. The constraints are partially due to the fundamental structure of the two-variable Green's functions, partially due to the empirically observed continuity of QMC selfenergy data (cf. Fig. 4.4).

\subsubsection{Functional-analytic consequences}

One can show that the continuity assumption for the real part at the branch point indeed leads to a complete description of the entire function $G(\underline{z})$ on all wedges only as a function of the single edge $\operatorname{Im} G\left(\underline{x}+\mathrm{i} 0^{\vartheta=0}\right)$. This is extraordinarily attractive from a computational point of view. The number of degrees of freedom within the maximum entropy method is not increased, but all imaginary-time theory data $G\left(\mathrm{i} \varphi_{m}, \mathrm{i} \omega_{n}\right)$ may be taken into account, without any a-priori constraint. We will also see that for functions which comply with the assumption, it in fact alleviates the 


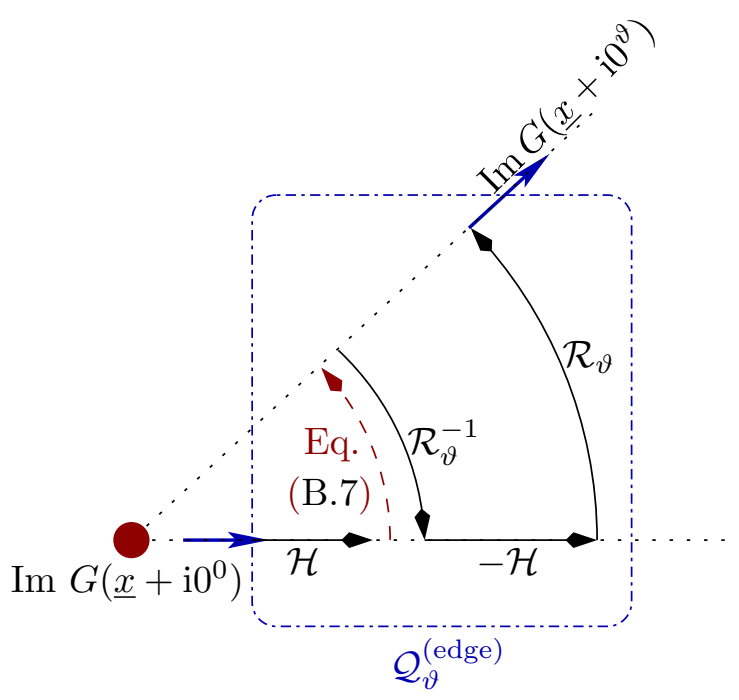

Figure 7.2: Sketch of the action of the operator $-\mathcal{Q}_{\vartheta}^{(\text {edge })}$.

severity of the inverse problem, as desired. On top of this, we still infer a positive definite function, which is the fundamental requirement of the MaxEnt method.

\section{Construction of the kernel}

If we take equation (B.7) for granted, we can derive a representation of the Green's function with respect to $\operatorname{Im} G\left(\underline{x}+\mathrm{i} 0^{\vartheta=0}\right)$ in the following way. First, we introduce the Hilbert transform operator $\mathcal{H}$ as

$$
(\mathcal{H} f)(\underline{x}):=\frac{1}{\pi} \oiint \mathrm{d} x_{2}^{\prime} \frac{f\left(x_{1}, x_{2}^{\prime}\right)}{x_{2}-x_{2}^{\prime}} .
$$

Then we can use condition 3' (section 5.3) in order to apply the Hilbert transform for computing real and imaginary part from each other on the boundary of certain $\mathbb{H}$-isomorphic complex lines.

One can show that

$$
\left.\operatorname{Im} G\right|_{T^{C_{r, \vartheta}}}=-\mathcal{Q}_{r, \vartheta} \cdot \operatorname{Im} G\left(\underline{x}+\mathrm{i} 0^{\vartheta=0}\right),
$$

where we introduced the operator

$$
\mathcal{Q}_{r, \vartheta}:=\mathcal{P}_{r, \vartheta} \mathcal{R}_{\vartheta} \mathcal{H} \mathcal{R}_{\vartheta}^{-1} \mathcal{H}
$$

Let us also introduce a symbol for the right part of the operator sequence,

$$
\mathcal{Q}_{\vartheta}^{(\text {edge })}:=\mathcal{R}_{\vartheta} \mathcal{H} \mathcal{R}_{\vartheta}^{-1} \mathcal{H}
$$


The action of $-\mathcal{Q}_{\vartheta}^{(\text {edge })}$ on $\operatorname{Im} G\left(\underline{x}+\mathrm{i} 0^{\vartheta=0}\right)$ is depicted in figure 7.2: First, the Hilbert transform $\mathcal{H}$ with respect to the $x_{\omega}$ variable yields $\operatorname{Re} G\left(\underline{x}+\mathrm{i} 0^{\vartheta=0}\right)$. Then, via equation (B.7) it is identified with $\operatorname{Re} G\left(\underline{x}+\mathrm{i} 0^{\vartheta}\right)$. In order to obtain $\operatorname{Im} G\left(\underline{x}+\mathrm{i} 0^{\vartheta}\right)$, one formally has to transform to the biholomorphic equivalent of $\left.G\right|_{T^{C_{r, \vartheta}}}$ in the domain $T^{C_{r, 0}}$ via the operator $\mathcal{R}_{\vartheta}^{-1}=\mathcal{R}_{-\vartheta}$. The inverse Hilbert transform $-\mathcal{H}$ yields the imaginary part of the edge value of the function $T^{C_{r, 0}} \rightarrow \mathbb{C}, \underline{z} \mapsto\left(\mathcal{R}_{\vartheta}^{-1}\left(\left.G\right|_{T^{C_{r, \vartheta}}}\right)\right)(\underline{z})$. Transforming the function back to the edge of $T^{C_{r, \vartheta}}$ using $\mathcal{R}_{\vartheta}$ yields the result $\operatorname{Im} G\left(\underline{x}+\mathrm{i} 0^{\vartheta}\right)$.

Using the Poisson kernel $\mathcal{P}_{r, \vartheta}$, Eq. (5.50), the Green's function is obtained in the desired wedge $T^{C_{r, \vartheta}}$. The entire procedure is contained in $\mathcal{Q}_{r, \vartheta}$.

\section{Feature of $\mathcal{Q}_{\vartheta}^{(\text {edge) }}$ : decoding branch cut geometry from single edge function}

The operator $\mathcal{Q}_{\vartheta}^{(\text {edge) }}$ is a map of pure "edge" character. Therefore, it seems worthwhile to study it separately. $\mathcal{Q}_{\vartheta}^{(\text {edge) }}$ is well-defined for any square-integrable function $f(\underline{x})$, no matter which orientation $\vartheta$ is considered.

Considering an edge function which is compatible with the Green's function properties $1,2,3^{\prime}$ (section 5.3), $\mathcal{Q}_{\vartheta}^{(\text {edge) }}$ is not defined for the singular orientations (5.56). For example, a straightforward calculation shows that applying $\mathcal{Q}_{\vartheta}^{(\text {edge })}$ step by step to $G_{0}\left(\underline{x}+\mathrm{i} 0^{\vartheta=0}\right)$ yields exactly the formula (5.52), with the switching behaviour (5.54) whose value is undefined for the orientations (5.56). The missing square-integrability of the edge functions along these directions is the corresponding mathematical reason. In particular, whenever $\mathcal{Q}_{\vartheta}^{(\text {edge) }}$ crosses a singular orientation of $\operatorname{Im} G_{0}\left(\underline{x}+\mathrm{i} 0^{\vartheta=0}\right)$, it exactly generates the jump in $\operatorname{Im} G_{0}\left(\underline{x}+\mathrm{i} 0^{\vartheta}\right)$ as a function of $\vartheta$.

Consequently, assuming (B.7) is correct, both, not only the holomorphic structure, but also the complete information about the entire branch cut structure, namely the exact geometry of the branch cuts, are encoded in the single edge function $\operatorname{Im} G(\underline{x}+$ $\left.\mathrm{i} 0^{0}\right)$. This correspondence is illustrated in figure 7.3. Same is true for the $(\vartheta=0)$ edge limit of the second-order self-energy, $\operatorname{Im} \Sigma^{(2)}\left(\underline{x}+\mathrm{i} 0^{0}\right)$, due to equation (7.2). It is always square-integrable, except for the directions $x_{\omega}=-3 / 2 x_{\varphi}, x_{\omega}=-x_{\varphi} / 2$, $x_{\omega}=x_{\varphi} / 2, x_{\omega}=3 / 2 x_{\varphi}$, namely for the geometry of the 2 nd-order branch cuts.

In the language of Shilov boundaries, the continuity constraint (B.7) of the considered function class caused a shrinking of the Shilov boundary as compared to the more general function class specified by points $1,2,3$ ' in section 5.3 .

Other properties of $\mathcal{Q}_{\vartheta}^{(\text {edge) }}$ are translational invariance, scale invariance, but in general no rotational invariance: For the translation operator

$$
\left(\mathcal{T}_{\underline{x}^{\prime}} f\right)(\underline{x}):=f\left(\underline{x}-\underline{x}^{\prime}\right)
$$

and for the homogenous scaling operator

$$
\left(\Lambda_{\lambda} f\right)(\underline{x}):=\lambda^{2} f(\lambda \underline{x}), \lambda>0
$$




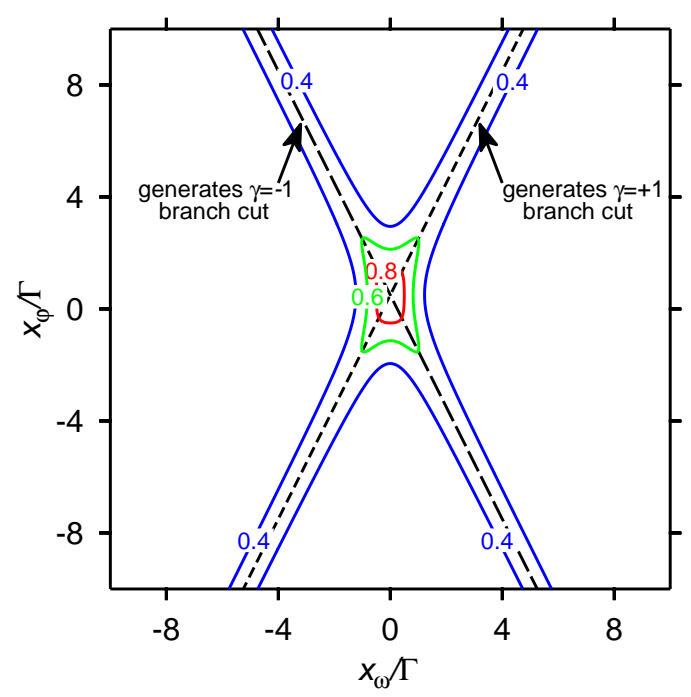

Figure 7.3: Plot of $\pi \tilde{A}_{0}(\underline{x})$ (equation (5.59), with $\varepsilon_{d}=0$ and $e \Phi=0.5 \Gamma$ ) showing correspondence of noninteracting branch cut structure (see figure 3.4 ) and $\tilde{A}_{0}$ function, as revealed by operator $\mathcal{Q}_{\vartheta}^{\text {(edge) }}$.

we have

$$
\begin{aligned}
& {\left[\mathcal{Q}_{\vartheta}^{\text {(edge) }}, \mathcal{T}_{\underline{x}}\right]=0} \\
& {\left[\mathcal{Q}_{\vartheta}^{\text {(edge) }}, \Lambda_{\lambda}\right]=0}
\end{aligned}
$$

but for the rotations

$$
\left[\mathcal{Q}_{\vartheta}^{\text {(edge) }}, \mathcal{R}_{\vartheta^{\prime}}\right] \neq 0
$$

on the set of functions we consider. The proof of these commutator relations is provided in appendix C. Note that because directional scaling implies a shear and therefore nonconserved angles in the shapes of $\operatorname{Im} G\left(\underline{x}+\mathrm{i} 0^{0}\right)$, it is no symmetry of the operator, in contrast to uniform scaling.

\subsubsection{Numerical implementation of $\mathcal{Q}_{r, \vartheta}$}

The numerical implementation of the kernel $\mathcal{Q}_{r, \vartheta}$ is unfortunately still quite cumbersome. Assuming that $G\left(\underline{x}+\mathrm{i} 0^{0}\right)$ is sufficiently smooth, we can represent it by superimposing localized test functions which span the space of edge functions. ${ }^{3}$

\footnotetext{
${ }^{3}$ Due to Bogolyubov's theorem (section 7.1), the smoothness of edge functions can indeed be rigorously proven for functions which are compatible with the assumptions of the theorem, because the resulting holomorphy and thus continuity on the edges. The symmetry relation (3.96) of the Green's function might help to satisfy these requirements.
} 


\section{Integral structure of the mapping}

$\mathcal{Q}_{r, \vartheta}$ is comprised of a quadruple integral. The first two integrals are the two principal value integrals which come with the Hilbert transforms. The second ones are included by the Poisson kernel $\mathcal{P}_{r, \vartheta}$. The integrations are formally very similar to a sequence of convolutions $A *(B *(C * e))$, where $e$ is an edge function. A crucial point is that due to the distributional nature of both, the principal values and the edge functions, the associativity rule cannot be expected to hold for these convolutions (see section 4.2 in Ref. [124]): Principal value and our type of edge functions (functions with singular directions, Eqs. (5.56)) are no distributions with compact support. Therefore, it is impossible to simply contract some "inner integrals" within $\mathcal{Q}_{r, \vartheta}$ analytically in order to obtain a simple kernel function for $\mathcal{Q}_{r, \vartheta}$. The use of a set of test functions which spans the space of edge functions is mandatory.

\section{Construction of the test functions}

The test functions would preferably be structured in a way which allows the quadruple integral in the operator $\mathcal{Q}_{r, \vartheta}$ to be solved essentially analytically. The functions

$$
f_{\underline{X}, \delta}:=\mathcal{T}_{\underline{X}} \Lambda_{1 / \delta} f
$$

with

$$
f(\underline{x}):=\frac{1}{\pi^{2}} \prod_{\alpha= \pm 1} \frac{1}{\left(x_{\omega}-\alpha x_{\varphi} / 2\right)^{2}+1}
$$

turn out to be a good starting point for this: First, we have the Dirac delta distribution

$$
\lim _{\delta \rightarrow 0} f_{\underline{X}, \delta}=\delta(\underline{x}-\underline{X})
$$

as a limit. Second, the use of scaling and translation operators yields the - regarding the action of the integrals in $\mathcal{Q}_{\vartheta}^{(\text {edge) }}$ - much more simple expression

$$
\left(\mathcal{Q}_{r, \vartheta} f_{\underline{X}, \delta}\right)(\underline{x})=\left(\mathcal{P}_{r, \vartheta} \mathcal{T}_{\underline{X}} \Lambda_{1 / \delta}\left(\mathcal{Q}_{\vartheta}^{(\text {edge })} f\right)\right)(\underline{x})
$$

rather than $\left(\mathcal{P}_{r, \vartheta}\left(\mathcal{Q}_{\vartheta}^{(\text {edge })} \mathcal{T}_{\underline{X}} \Lambda_{1 / \delta} f\right)\right)(\underline{x})$ as a matrix element of $\mathcal{Q}_{r, \vartheta}$. Third, the simple pole structure of (7.18) allows us to compute most of the integrals analytically. Note that the simpler looking symmetric Lorentzian function $\frac{1}{x_{\omega}^{2}+x_{\varphi}^{2}+1}$ is, in fact, no good alternative to $f$, because the poles with respect to $z_{\omega}$ or $z_{\varphi}$ contain square roots of $z_{\varphi}$ or $z_{\omega}$, respectively. Similar problems arise for localized Gaussians.

The directional arbitrariness $x_{\omega} \pm x_{\varphi} / 2$ arising in Eq. (7.18) from choosing a product of 1-dimensional Lorentzians in (7.18) is still to be discussed. For example, one could also have chosen it to be $x_{\omega} \pm x_{\varphi}$, adjusting the normalization factor from $\frac{1}{\pi^{2}}$ to $\frac{2}{\pi^{2}}$ in order to assert (7.19). A conceptional advantage of our choice of $f$ is, however, that for any domain $T^{C_{r, \vartheta}}$, for which $G$ is holomorphic, we have $r \leq 2$. Consequently, due 


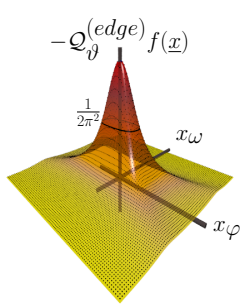

(a) $\vartheta=0$

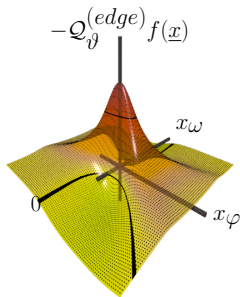

(b) $\vartheta=\pi / 4$

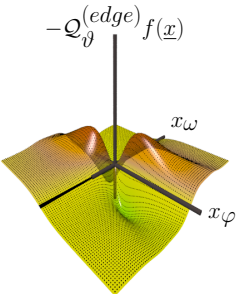

(c) $\vartheta=\pi / 2$

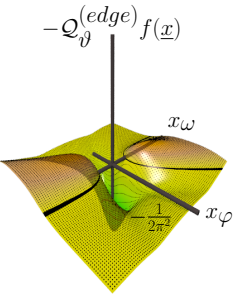

(d) $\vartheta=3 \pi / 4$

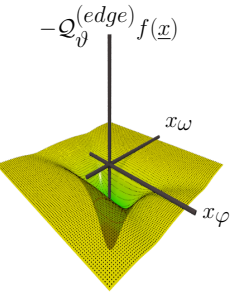

(e) $\vartheta=\pi$

Figure 7.4: Transformation behaviour of the test function $f(\underline{x})$ as a function of the edgeto-edge map $-\mathcal{Q}_{\vartheta}^{(\text {edge })}$ for different values of $\vartheta$. Function values are shown within the range $[-5,5] \times[-5,5]$.

to the pole structure of (7.18), $f$ is holomorphic in the domain $T^{C_{r, 0}} \subset T^{C_{2,0}}$, whose edge is the starting point of the $\mathcal{Q}_{r, \vartheta}$ transform. Nevertheless, a certain ambiguity remains which might be even technically useful.

\section{Computation of the matrix elements}

We found it feasible to calculate at least the first three integrals of the right-hand side of expression (7.20) analytically, using a computer algebra system. In order to compute the fourth integral, an adaptive numerical quadrature can be used.

The result of the analytical integration of the first two integrals, i.e. $\left(-\mathcal{Q}_{\vartheta}^{(\text {edge })} f\right)(\underline{x})$ is shown in figure 7.4 for selected edge orientations. We find that $\left(-\mathcal{Q}_{\vartheta}^{\text {(edge) }} f\right)(\underline{x})$ is a rational function which changes continuously as a function of $\vartheta$, in contrast to the transformation behaviour of $\operatorname{Im} G_{0}\left(\underline{x}+\mathrm{i} 0^{0}\right)$. Note that since $\mathcal{Q}_{\vartheta}^{(\text {edge) }}$ is scale-invariant, Eq. (7.15), the transformation behaviour of the Dirac delta distribution is analogous to figure 7.4. Consequently, the transformed delta distribution on the edge $\mathbb{R}+\mathrm{i} 0^{\vartheta}$ is not a function but rather a distribution with a relatively complicated structure. Therefore, the limit $\delta \rightarrow 0$ in (7.20) cannot be taken before the last two integrals from the Poisson kernel $\mathcal{P}_{r, \vartheta}$ are computed. In the special case $\vartheta=\pi / 2$, figure (7.4c), the asymptotic behaviour of the result decays $\propto \frac{1}{|\underline{x}|}$ when $\underline{x} \rightarrow \infty$, in contrast to the original test function behaviour $f(\underline{x}) \propto \frac{1}{|\underline{x}|^{2}}$. This is because the Hilbert transforms are taken with respect to mutually orthogonal directions in $\mathbb{R}^{2}$ here. This case yields the particularly simple expression

$$
\begin{aligned}
-\mathcal{Q}_{\vartheta=\pi / 2}^{(\text {edge })} f(\underline{x})= & \frac{1}{\pi^{2}} \cdot \frac{x_{\omega} x_{\varphi}}{\left(x_{\omega}^{2}+1\right)\left(x_{\varphi}^{2}+4\right)} . \\
& \cdot \frac{12 x_{\varphi}^{2}+x_{\varphi}{ }^{4}+16 x_{\omega}{ }^{4}+48 x_{\omega}{ }^{2}-8 x_{\varphi}{ }^{2} x_{\omega}{ }^{2}+96}{\left(x_{\varphi}{ }^{2}+4-4 x_{\varphi} x_{\omega}+4 x_{\omega}{ }^{2}\right)\left(x_{\varphi}{ }^{2}+4 x_{\varphi} x_{\omega}+4 x_{\omega}{ }^{2}+4\right)} .
\end{aligned}
$$


The angles between 0 and $\pi / 2$ interpolate smoothly between the extremal cases of the unperturbed well-localized $f(\underline{x})$ at $\vartheta=0$ and the long-range function (7.21) at $\vartheta=\pi / 2$ and involve higher-order rational functions. The solution at $\vartheta=\pi$ is again strongly localized and equals $-f(\underline{x})$. The behaviour in the interval $[\pi, 2 \pi)$ is analogous due to symmetry reasons.

\section{Implementation}

As mentioned above, the third integral, with respect to the $x_{1}$ variable, can still be computed analytically. However, each integration of the operator sequence (7.20) adds additional poles to the resulting function, and more and more complex distinctions have to be done in order to decide whether a pole is on the upper or lower half-plane and whether it contributes or not to certain residue sums.

Because translational and scale invariance do not seem to be as useful concepts when applied to $\mathcal{P}_{r, \vartheta}$, not only the extra variable $r$ appears in the computation of the remaining expressions, but also the shift $\underline{X}$ and the scale $\lambda$ of the test function (7.17). For the third integral, one can still determine the poles and residues before doing the latter substitution with the computer algebra system, however.

Doing this, with the computer algebra system at hand, the resulting symbolic expression for the integrand of the fourth integral coming from the Poisson kernel, approaches an approximate file size of 500 kilobytes. Due to a similar behaviour as that of the transformed edge functions, taking the limit $\delta \rightarrow 0$ is still not allowed for the integrand of the fourth integral.

An algebraic determination of the poles of this expression is cumbersome, because high-order polynomials appear in the denominator of the resulting expressions. Nevertheless, numerical computations indicate that the limit $\delta \rightarrow 0$ yields well-defined functions after the fourth integration. Once an algebraic expression is found, the expression for the limit $\delta \rightarrow 0$ would be more simple than the intermediate terms.

As already stated in the beginning of this section, we compute the fourth integral with an adaptive numerical integration routine in practice. One can adjust $\delta$ as a function of $\underline{x}$, depending on how well a specific region of the edge should be resolved. In future applications, the analytic evaluation of the fourth integral and the limiting process should provide a great simplification.

Implementation details of the resulting MaxEnt procedure are specified in appendix D. The numerical computation of the matrix elements is described in appendix E.

\subsection{Results of the $\mathcal{Q}$-approach}

When defined according to the interacting branch cut geometry, we will refer to $\mathcal{Q}_{r, \vartheta}$ simply by the symbol $\mathcal{Q}$. In order to obtain estimates for the finite- $U$ Anderson impurity model out of equilibrium, we determine a most probable default model 
(D.3),

$$
\tilde{D}_{\sigma_{\text {def }}}\left(x_{\varphi}, x_{\omega}\right)=\frac{1}{2 \pi} \sum_{\alpha= \pm 1} \frac{\sigma_{\text {def }}}{\left(x_{\omega}-\frac{\alpha}{2}\left(x_{\varphi}-\Phi\right)\right)^{2}+\sigma_{\text {def }}^{2}},
$$

first and, in a second step, refine the knowledge about its low-frequency region, with an extra low-energy width, and a transient behaviour as in equation (D.4),

$$
\sigma_{\text {def }}\left(x_{\varphi}\right)=\Gamma+\left(\tilde{\sigma}_{\text {def }}-\Gamma\right) \frac{R^{2}}{x_{\varphi}^{2}+R^{2}},
$$

where we set $R=5 \Gamma$. Due to the approximative nature of $\mathcal{Q}$, also the range of the data $G\left(\mathrm{i} \varphi_{m}, \mathrm{i} \omega_{n}\right)$ has to be constrained in order to obtain spectra with a normalization $\approx 1$. One finds empirically that a major constraint is $\omega_{n}>\left|\varphi_{m} / 2\right|$ for finite values of $U$. By this, the data space is in fact originated in the neighbouring wedges of the retarded Green's function's wedge. It is however very large as compared to the data space of the single-wedge approach as used in section 6.2.1. Furthermore, as many-body correlations increase, small values of $\omega_{n}$ must be neglected in order to apply $\mathcal{Q}$. This is in accordance with the observation that $\mathcal{Q}$ is only perturbatively correct.

It will be shown explicitly that the extended data range makes the inverse problem and also the MaxEnt procedure more well-behaved as compared to the single-wedge approach. Results of spectral functions and transport properties are presented in the weak- to intermediate coupling regime.

\subsubsection{Weak-Coupling Regime}

In the weak-coupling regime, $U \leq \pi \Gamma$, the second-order perturbation theory in $U / \Gamma$ is still expected to be rather accurate. A useful formula for the calculation of secondorder perturbation spectra at $T=0$ is provided by Ref. [126] for comparison purposes.

Already in the weak-coupling regime, $U=2 \Gamma, \beta \Gamma=5$, the applicability of the $\mathcal{Q}$ approach is limited a posteriori by bad behaviour of the inferred spectral functions precisely to input data with $\omega_{n}>\left|\varphi_{m} / 2\right|$. If any data point $G\left(\mathrm{i} \varphi_{m}, \mathrm{i} \omega_{n}\right)$ with $\omega_{n}<$ $\left|\varphi_{m} / 2\right|$ is included, the MaxEnt procedure ceases to converge to a reasonable solution. This corresponds to not crossing the principal branch cut $\gamma= \pm 1$ in figure 3.4, when coming from the retarded Green's function. Apart from this, there appears to be no further constraint. We use all Matsubara data $\omega_{n}$, extending from $n=1$ to $n=8$, while $m= \pm 1, \ldots, \pm 5$ in $\varphi_{m}$, with the $\omega_{n}>\left|\varphi_{m} / 2\right|$ constraint for $\omega_{n}$.

As a first test, figure 7.5 displays the performance of the MaxEnt method for both, the single-wedge and the multiple-wedge approach for the given data set. The singlewedge approach implicitly assumes the interacting Green's function to be analytic for $\operatorname{Im} z_{\omega}>\left|\operatorname{Im} z_{\varphi} / 2\right|$. Apparently, this wrong assumption makes no fit with a positive definite $\tilde{A}(\underline{x})$ possible. Instead, the $\chi^{2}$ value does not drop below $\approx 10^{6}$, and the 


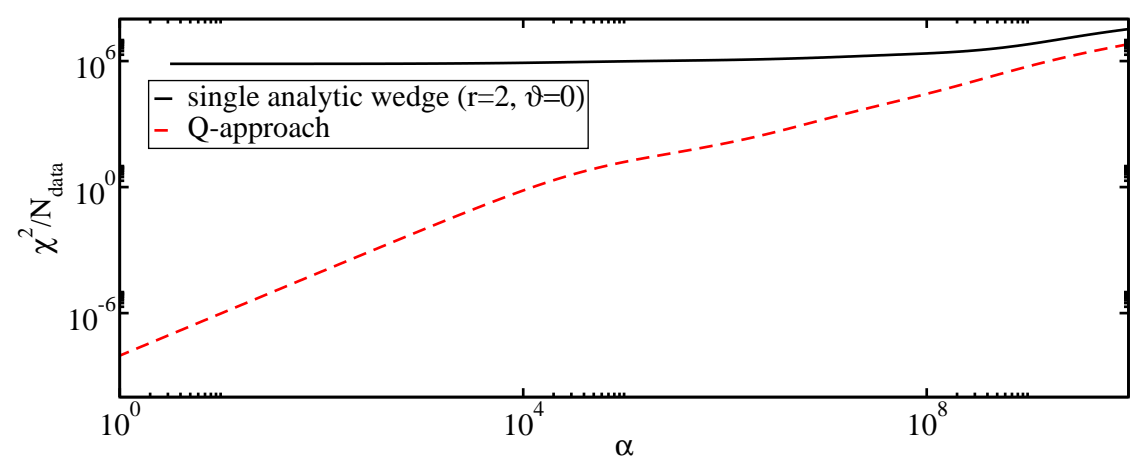

Figure 7.5: Comparison of $\chi^{2}$ in the MaxEnt for single-wedge kernel $\mathcal{P}_{r, \vartheta}$ and multi-wedge kernel $\mathcal{Q}$ at $U=2 \Gamma, \beta=5 \Gamma^{-1}$, and $e \Phi=\Gamma$ as a function of the regularization parameter $\alpha$. For the same input set, the single-wedge approach already clearly fails to converge due to the wrong assumption of negligible branch cuts $|\gamma| \geq 3$.

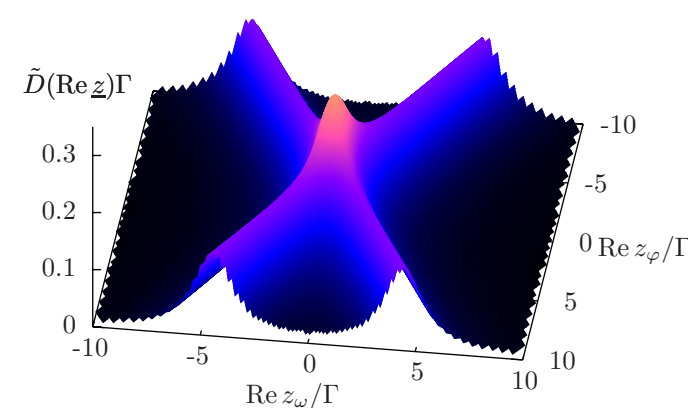

(a) a-posteriori identified best default model

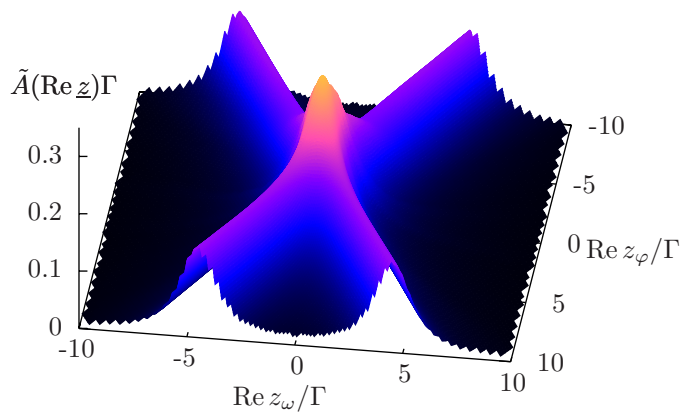

(b) inferred $\tilde{A}(\underline{x})$

Figure 7.6: Application of the MaxEnt procedure for the $\mathcal{Q}$-mapping to the nonequilibrium weak-coupling case $U=2 \Gamma, \beta=5 \Gamma^{-1}, e \Phi=\Gamma$, with CT-QMC data as input. The default model has been identified via its maximal posterior probability.

MaxEnt does not converge. In sharp contrast, values of $\chi^{2} / N_{\text {data }} \approx 1$ may be reached with the MaxEnt with respect to $\mathcal{Q}$. Also, controls such as the MaxEnt error rescaling merit do not indicate the presence of any abnormalities.

For the $\mathcal{Q}$-mapping, a well-behaved MaxEnt solution is obtained. Applying the procedure described in appendix D, the edge function displayed in figure 7.6a is identified as a-posteriori most probable default model. Using this default model, the edge function in panel $7.6 \mathrm{~b}$ is obtained. An overall moderate sharpening of the edge function along the cross-like structure is observed.

The resulting spectral function is displayed in figure 7.7 together with the spectral function of the default model which has been identified as most probable a posteriori. The spectrum is in good agreement with the zero-temperature second-order perturbation theory. Presumably due to the finite temperature, the quasi-particle weight is slightly smaller than the perturbative one. 


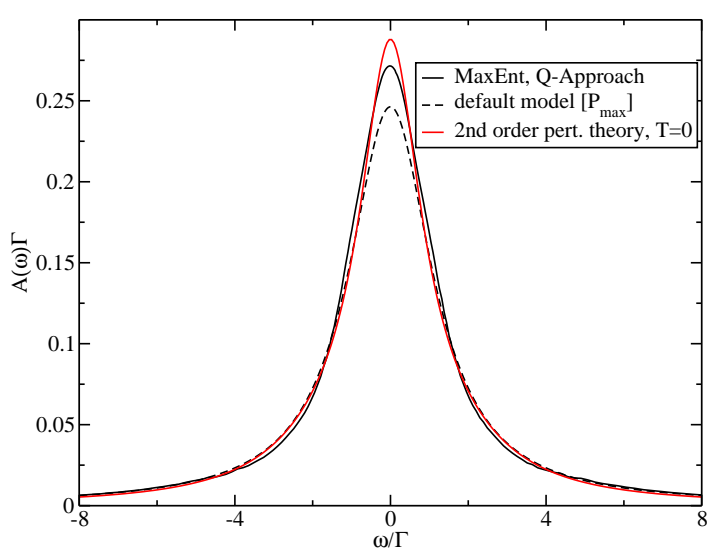

Figure 7.7: Spectral function as inferred for the nonequilibrium weak-coupling case $U=2 \Gamma$, $e \Phi=\Gamma, \beta=5 \Gamma^{-1}$, compared to zero-temperature second-order perturbation theory.

\subsubsection{Intermediate-Coupling Regime}

Using the same range within Matsubara space, $\omega_{n}>\left|\varphi_{m} / 2\right|$, the method can well be applied to intermediate coupling strengths. At the comparably small inverse temperature $\beta=5 \Gamma^{-1}$, calculations are most readily done, mainly due to the comparably small QMC data space of approximately 50 imaginary-time-theory data points. In general, the amount of data will grow quadratically as a function of inverse temperature, due to the simultaneous presence of Matsubara voltage and Matsubara frequency. Additionally, at low temperatures, sharp features in the spectral function and hence the $\tilde{A}$ will have to be resolved, requiring an enhanced grid refinement. Altogether, matrix sizes in the MaxEnt are blown up substantially when the temperature is decreased. In particular, the computational effort for the generation of an appropriate kernel matrix (cf. appendix E) is increased by this. Apart from this presumably, in principle, minor technical issue, the memory consumption of the MaxEnt itself poses a limitation at lower temperatures at the current stage of development. Additionally, it is well-known that the resolution of low-temperature features with the MaxEnt method requires a careful Bayesian analysis based on higher-temperature data, i.e. an "annealing procedure" involving many higher-temperature data [35; 127].

Figure 7.8 shows some spectral functions obtained within the intermediate-coupling regime at $\beta=5 \Gamma^{-1}$, but without imposing any annealing procedure. In parts, the spectral function has not been modified by the MaxEnt, but remains attached to the default model. The agreement in shape and height with second-order zerotemperature perturbation theory seems satisfactory.

However, in particular in the cases $(U=4 \Gamma, e \Phi=0.125 \Gamma),(U=4 \Gamma, e \Phi=0.25 \Gamma)$, and $(U=6 \Gamma, e \Phi=0.25 \Gamma)$, the formation of peculiar side-bands can be observed, coming along with an unphysical increase in the spectral sum. They are clearly an artifact of the continuation procedure. The resulting unphysical region however seems 
to be limited to a certain energy range which is not necessarily of physical interest. It can often be traced back to certain structures which seem to form along well-defined angular directions in the function $\tilde{A}\left(x_{\varphi}, x_{\omega}\right)$. For the case $(U=6 \Gamma, e \Phi=0.25 \Gamma)$, the MaxEnt inference process is displayed in figure 7.9. The quasi-particle resonance is generated by the peak at $\underline{x} \approx 0$. The formation of broad Hubbard shoulders is accompanied by the structures producing the unphysical sidebands.

A comparison to scattering-states numerical renormalization group (SNRG) spectra is shown in figure $7.10 .{ }^{4}$ While data seem to be compatible with a splitting of the quasi-particle resonance, i.e. peaks at $\pm e \Phi / 2$, the SNRG does not exhibit this feature. The double-peak structure is found to be generated by the Matsubara data points in the vicinity of the edge, i.e. the low-energy imaginary-time theory. Due to the assumption which the $\mathcal{Q}$-approach relies on, it cannot yet be decided whether the prediction of the quasi-particle peak to split is a prediction of Matsubara voltage theory or is an artifact of the $\mathcal{Q}$-approach. The peak-splitting could also be generated as an artifact of the MaxEnt procedure - however, the rather systematic positions at $\pm e \Phi / 2$ oppose against this hypothesis.

\subsubsection{Transport properties}

Due to the increased quality of spectral functions, the calculation of transport properties is much more reliable with the multi-wedge approach than with the single-wedge continuation. In figure 7.11, resulting electric currents are compared to real-time quantum Monte-Carlo data by Werner et al. [32]. The results for $\beta=5 \Gamma^{-1}$ are presumably more reliable than at lower temperature $\beta=10 \Gamma^{-1}$. A good agreement with the real-time QMC data is found. The extraordinarily high value at $\beta=10 \Gamma^{-1}$ and $U=6 \Gamma$ for voltage $e \Phi=0.0675 \Gamma$ can be traced back to an unphysically high quasi-particle peak, which may however still be resulting from stochastic noise or imperfections of the MaxEnt procedure. The latter is again more demanding for $\beta=10 \Gamma^{-1}$ than for $\beta=5 \Gamma^{-1}$. Figure 7.12 shows the current as a function of the voltage for several values of $U$.

\subsection{Perspective: Exact $\mathcal{Q}$-approach}

The underlying continuity assumption of the $\mathcal{Q}$-approach is only approximate, because in higher order of perturbation theory, terms (B.1) which do not characterize the collection of wedges, but merely just isolated wedges, are generated. These terms are manifested in discontinuities of the real part of the Green's function at the branch point $\operatorname{Im} \underline{z}=0$.

In order to extend the $\mathcal{Q}$-approach to the full nonequilibrium Kondo regime $U \geq$ $2 \pi \Gamma, e \Phi \sim T_{K}, \beta^{-1} \sim T_{K}$, one has to take these contributions into account. This

\footnotetext{
${ }^{4}$ SNRG data were provided by Sebastian Schmitt and Frithjof Anders. Used with permission.
} 


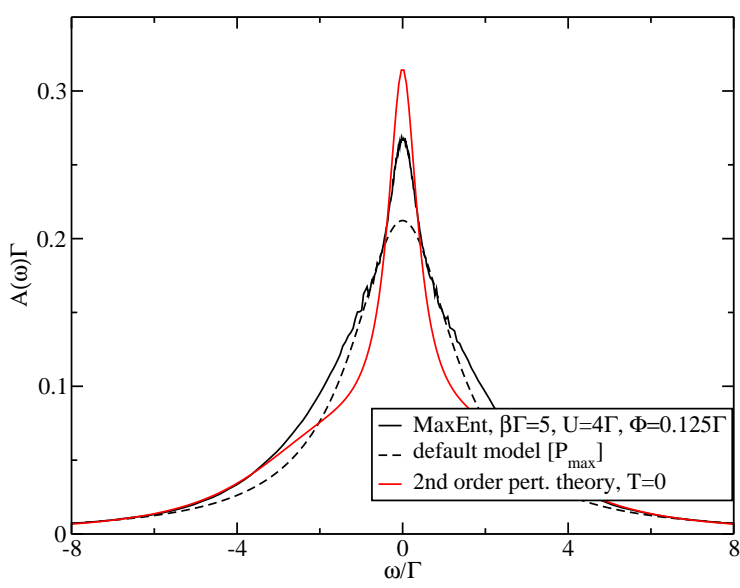

(a) $U=4 \Gamma, e \Phi=0.125 \Gamma$

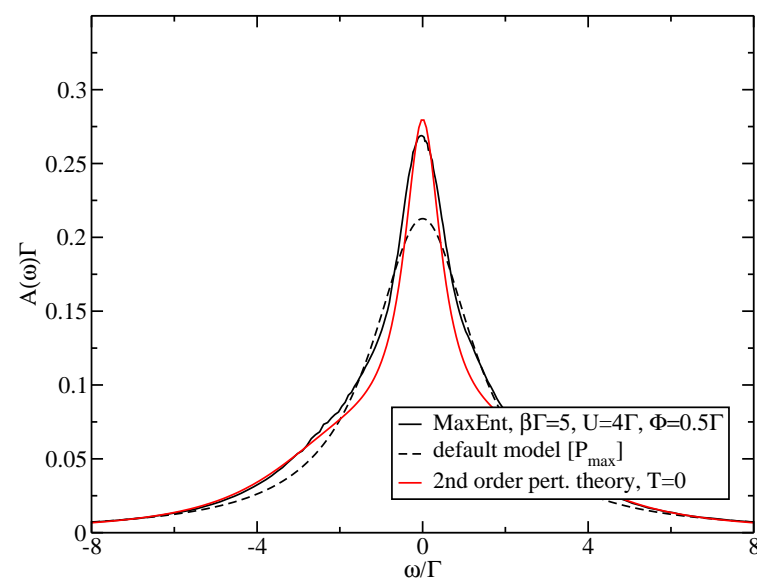

(c) $U=4 \Gamma, e \Phi=0.5 \Gamma$

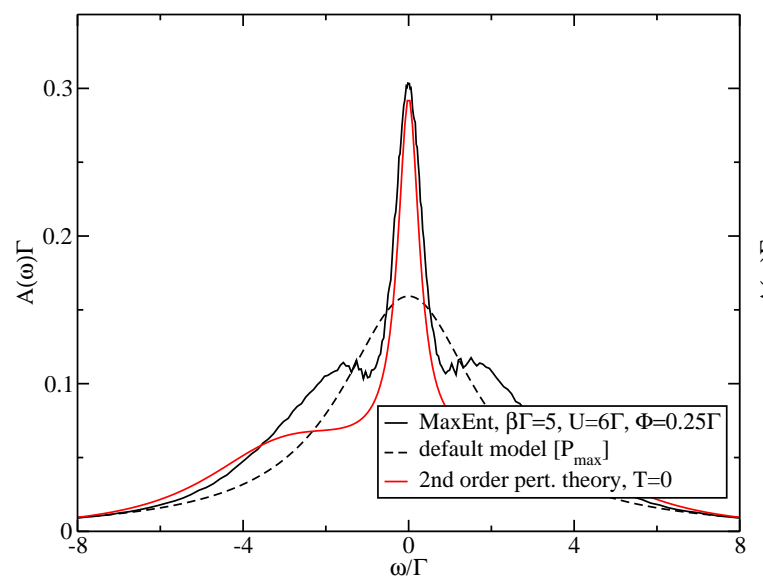

(e) $U=6 \Gamma, e \Phi=0.25 \Gamma$

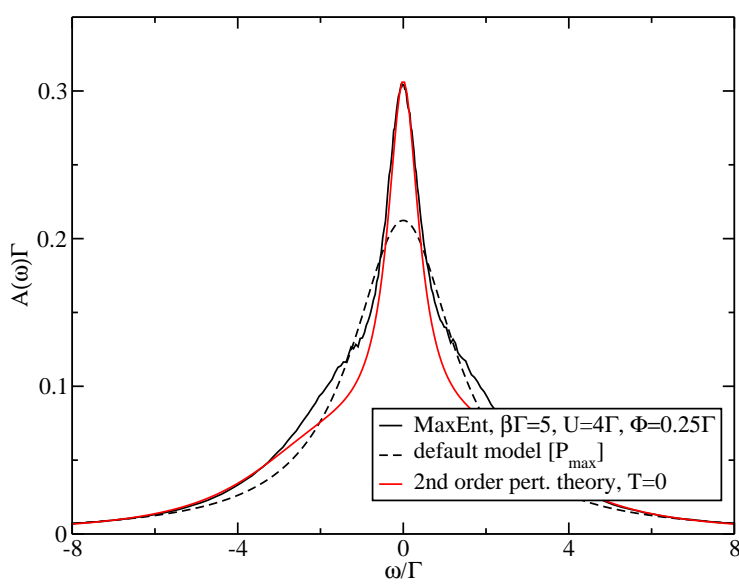

(b) $U=4 \Gamma, e \Phi=0.25 \Gamma$

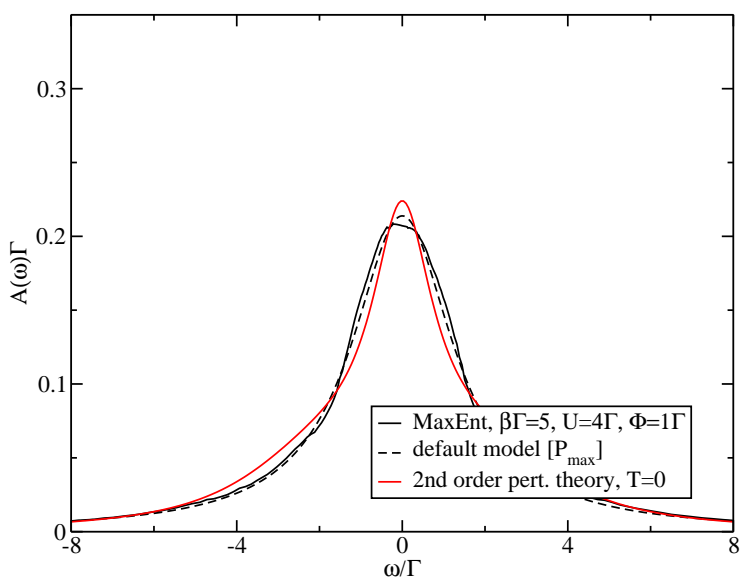

(d) $U=4 \Gamma, e \Phi=1.0 \Gamma$

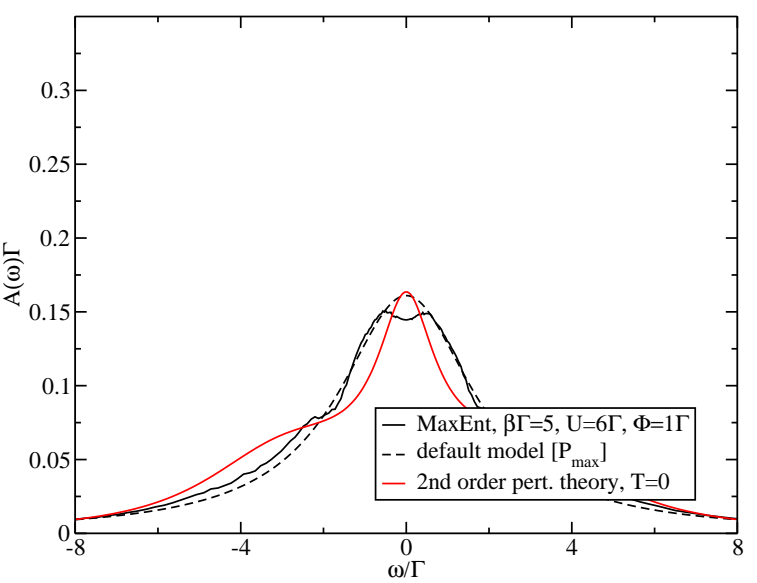

(f) $U=6 \Gamma, e \Phi=1.0 \Gamma$

Figure 7.8: Intermediate-coupling nonequilibrium spectral functions at inverse temperature $\beta=5 \Gamma^{-1}$, as compared to zero-temperature second-order perturbation theory. 


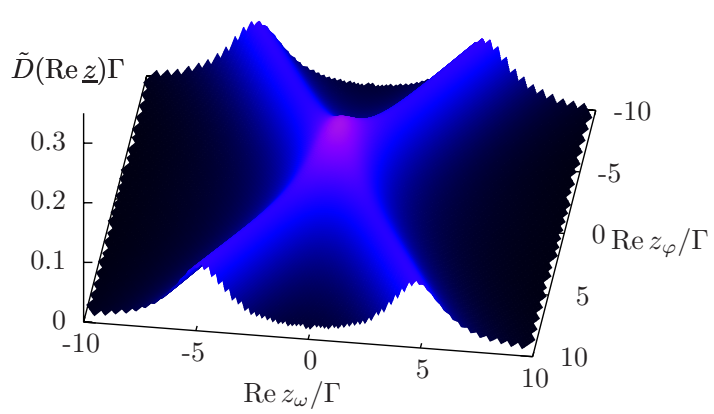

(a) a-posteriori identified best default model

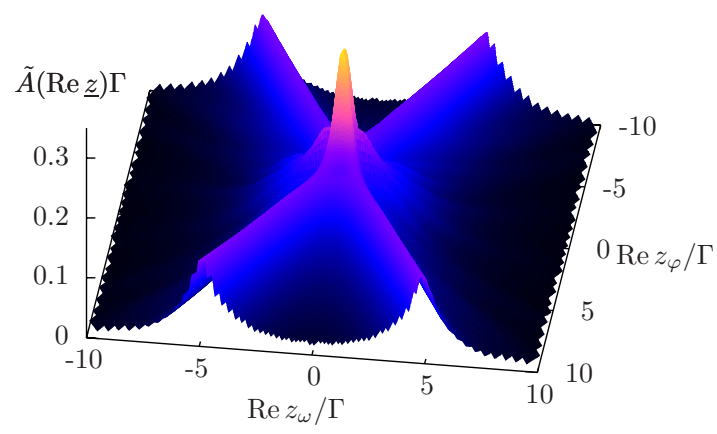

(b) inferred $\tilde{A}\left(x_{\varphi}, x_{\omega}\right)$ function

Figure 7.9: Bayesian inference process for $U=6 \Gamma, e \Phi=0.25 \Gamma, \beta=5 \Gamma^{-1}$.

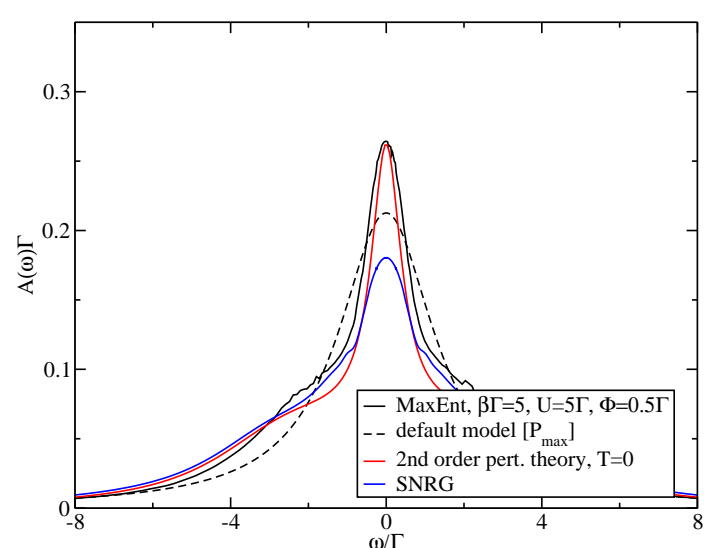

(a) $U=5 \Gamma, e \Phi=0.5 \Gamma, \beta=5 \Gamma^{-1}$

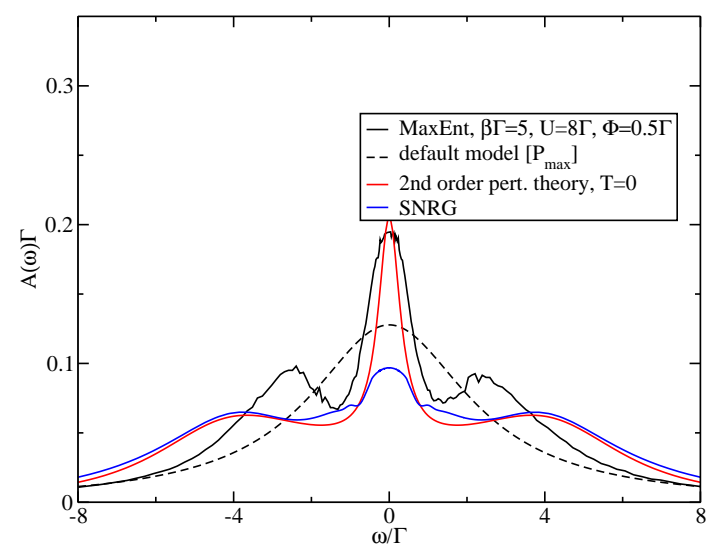

(c) $U=8 \Gamma, e \Phi=0.5 \Gamma, \beta=5 \Gamma^{-1}$

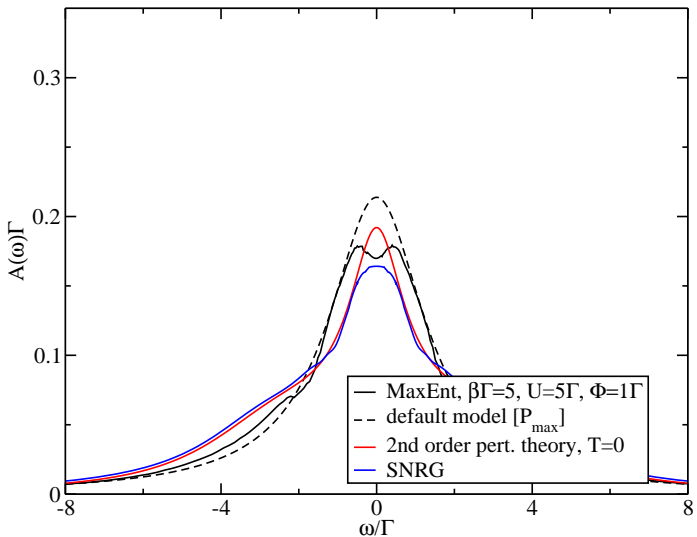

(b) $U=5 \Gamma, e \Phi=1.0 \Gamma, \beta=5 \Gamma^{-1}$

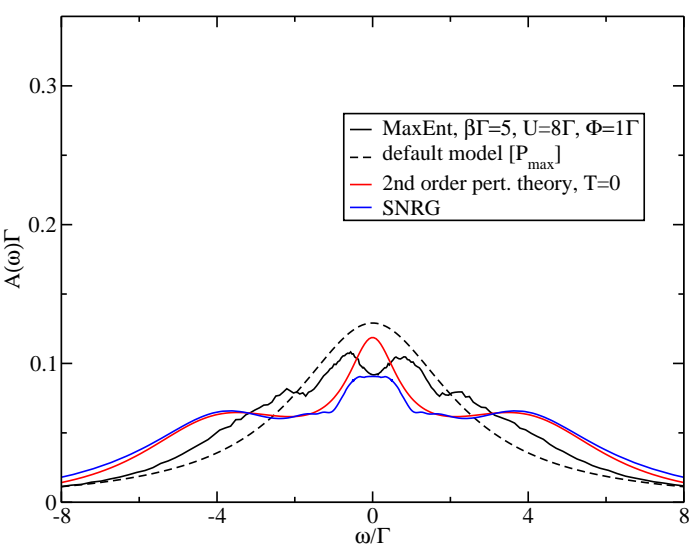

(d) $U=8 \Gamma, e \Phi=1.0 \Gamma, \beta=5 \Gamma^{-1}$

Figure 7.10: Comparison to the scattering-states numerical renormalization group 


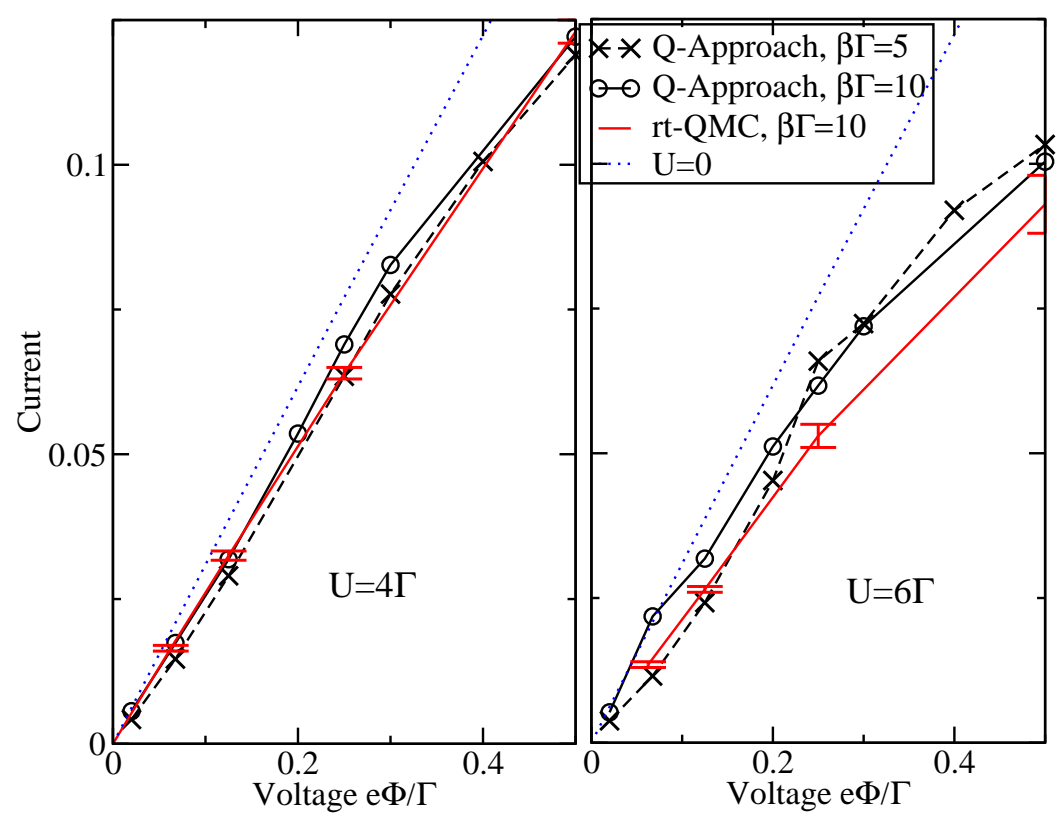

Figure 7.11: Currents for $U=4 \Gamma$ and $U=6 \Gamma$ as compared to real-time QMC [32] and the noninteracting case.

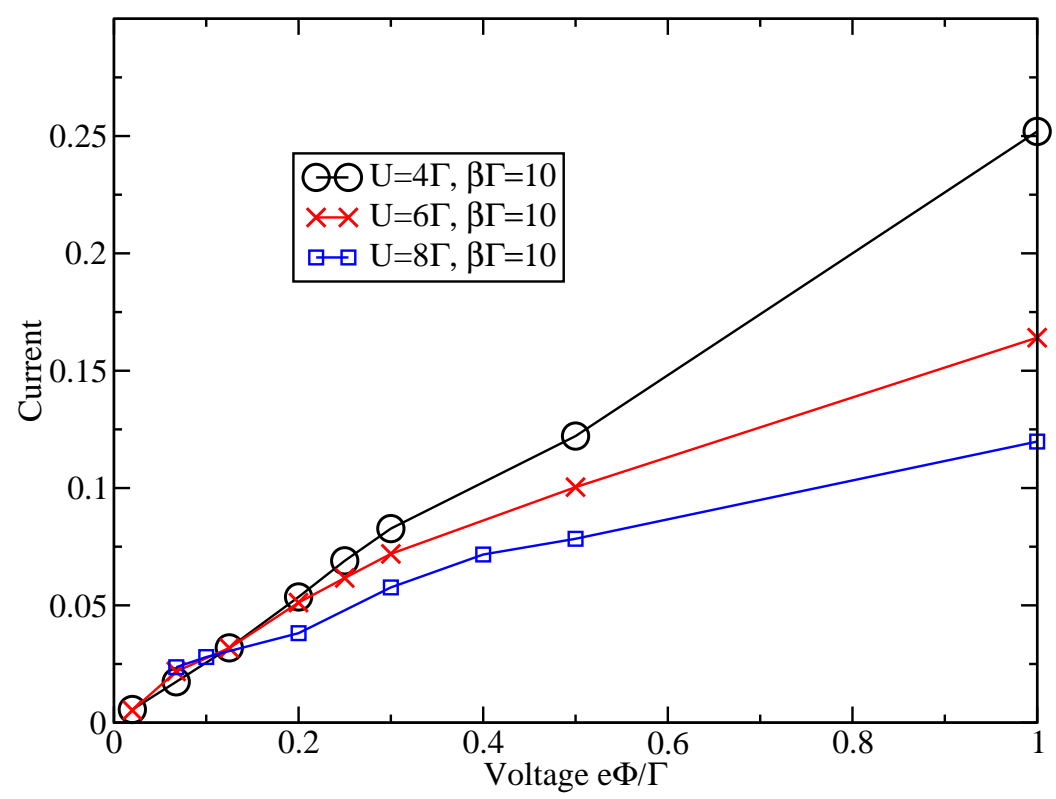

Figure 7.12: Current as a function of voltage at $\beta=10 \Gamma^{-1}$ for several interaction strengths. 
requires the consideration of the full analytic structure of the theory as illustrated in figure $7.13 \mathrm{~b}$. As a consequence, extra terms have to be added to the representation of $G\left(z_{\varphi}, z_{\omega}\right)$ within the MaxEnt procedure. Natural candidates for such degrees of freedom are the residual imaginary parts of edge functions

$$
\tilde{R}_{\nu}(\underline{x}):=\operatorname{Im} G\left(\underline{x}+\mathrm{i} 0^{\vartheta_{\nu}}\right)-\pi \cdot\left(\mathcal{Q}_{\vartheta_{\nu}}^{(\text {edge })} \tilde{A}\right)(\underline{x}),
$$

for the edge of the $\nu$-th wedge with orientation $\vartheta_{\nu}$. Due to the properties of the residual terms (B.1), $\tilde{R}_{\nu}(\underline{x})$ is expected to vanish as $\underline{x} \rightarrow \infty$. I.e. the functions are essentially localized within a finite radius around 0 which can be expected to be of the order of magnitude of the energy scales $\Gamma, U, \varepsilon_{d}$, and $e \Phi$. One can therefore hope that although one formally introduces infinitely many two-dimensional variable vectors to take into account the exact analytic structure, in the actual discretized version of the problem, the growth in variable space is not so dramatic. This must be seen in relation to the $\tilde{A}$ function, which has to be discretized over a very wide range in order to pay tribute to the strong intertwining of edge function structure and branch cut structure.

For data in the $\nu$-th wedge, one then has the exact equation

$$
\operatorname{Im} G\left(\mathrm{i} \varphi_{m}, \mathrm{i} \omega_{n}\right)=\left(\mathcal{P}_{r_{\nu}, \vartheta_{\nu}} \tilde{R}_{\nu}\right)\left(\mathrm{i} \varphi_{m}, \mathrm{i} \omega_{n}\right)+\pi \cdot(\mathcal{Q} \tilde{A})\left(\mathrm{i} \varphi_{m}, \mathrm{i} \omega_{n}\right),
$$

where $r_{\nu}$ is the opening ratio of the wedge. ${ }^{5}$ The inverse problem to be solved by the MaxEnt is to determine $\tilde{R}_{\nu}$ and $\tilde{A}$ simultaneously. Practically, the terms $\tilde{R}_{\nu}$ would act as "valves" for the conceptual imperfection of the $\mathcal{Q}$-mapping within the Bayesian information flow.

Because the functions $\tilde{R}_{\nu}(\underline{x})$ cannot be expected to be positive, it is necessary to introduce a shift to a positive function, such as for the spectral functions of the static observables in section 6.1. The terms $\tilde{R}_{\nu}(\underline{x})$ are most dominant for wedges next to the noninteracting Green's function's branch cuts. A very careful Bayesian analysis, including an appropriate set of choosable default models constructed from a-priori information, is probably required for a successful application of the exact approach. ${ }^{6}$

\subsection{Summary}

In this chapter, the MaxEnt approach to the double analytic continuation was systematically extended to a substantially larger range of QMC simulation data. At the cost of an approximation to the structure at the branch point, ${ }^{7}$ it enabled to estimate dynamical properties up to the intermediate coupling regime which are in reasonable agreement with other computational techniques.

\footnotetext{
${ }^{5}$ For the definitions see figure 5.4.

${ }^{6}$ Some a-priori information is provided in appendix B.

${ }^{7}$ We refer to the point where all wedges join as branch point.
} 


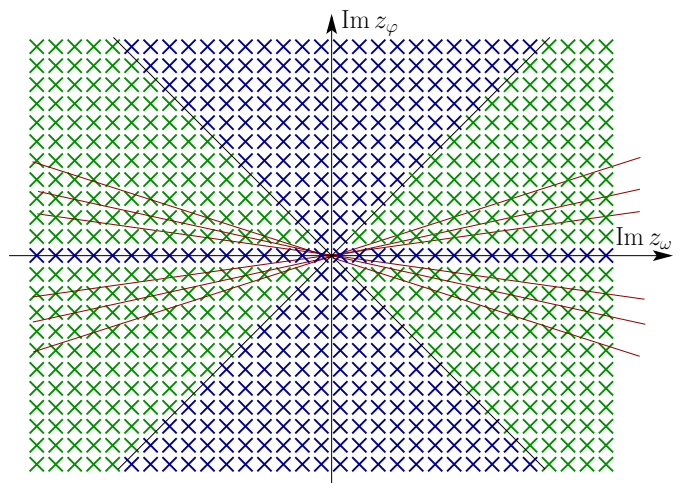

(a) imaginary-voltage formalism data

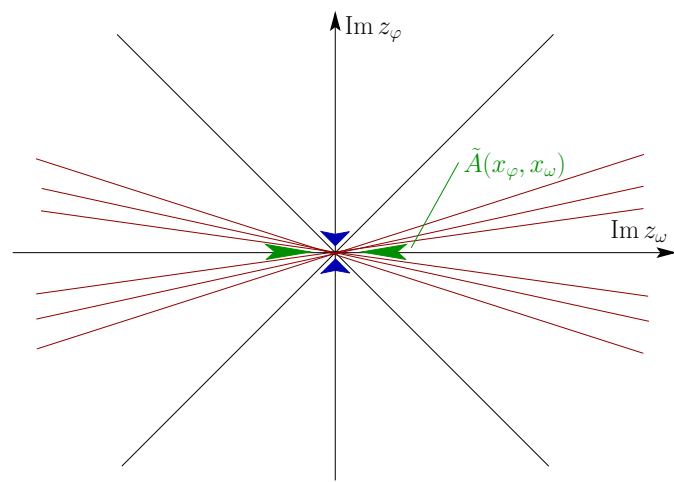

(b) $\mathcal{Q}$-simplified set of edge functions

Figure 7.13: Effective simplification of the analytic structure as found a posteriori for the $\mathcal{Q}$-mapping. It is valid up to intermediate many-body correlation strengths. Compare to the general structure depicted in figure 7.1. The inaccessibility of the upper and lower wedges to $\mathcal{Q}$ is presumably due to the strong violation of the shared-real-part assumption within the multiplicative structure of the Dyson series, as predicted as an a-priori constraint by section 7.2.2. In strong contrast to the single-wedge approach, the $\varphi_{m}=0$ data are not used, because they are located on the accumulation axis of interacting branch cuts and also on the $\operatorname{Im} z_{\varphi}=0$ branch cut.

Resulting from an a-posteriori constraint, the accessible data range was extended to the one symbolized by the set of green crosses in figure 7.13. The mathematical setup is much simpler than the nonapproximate analytic structure sketched in figure 7.1. The technical realization of a matrix representation for the resulting mapping $\mathcal{Q}$ and the Bayesian inference procedure, however, required a carefully prepared computational procedure.

As a future application, an exact generalization of the approach was suggested. It introduces additional degrees of freedom from several distinct edge functions which have to be determined sufficiently accurately by the MaxEnt procedure. Along the route to the ultimate goal of providing estimates on non-equilibrium strong-coupling spectra, in particular the identification of appropriate a-priori information for incorporation into the default model are expected to be a non-trivial task. 
7. Generalized Approach to the Double Analytic Continuation 


\section{Conclusion}

The Matsubara approach to the Anderson impurity model out of equilibrium by Han and Heary was studied regarding its viability. Particular emphasis was put on the computational feasibility of numerically controlled predictions.

We showed that highly accurate unbiased imaginary-time data can be produced for the resulting effective-equilibrium systems in the Kondo limit by means of recently developed continuous-time quantum Monte-Carlo methods. The auxiliary-field expansion algorithm (CT-AUX) was identified to be particularly useful for this purpose.

Based on the perturbative structure of the theory, an exact analytic continuation formula for certain types of static observables was derived, namely the double occupancy and the magnetization of the quantum dot. Employing a MaxEnt procedure, non-trivial predictions resulted which are in reasonable agreement with other computational methods such as the scattering-states numerical renormalization group (SNRG). The simulation data were found to be fully consistent with the formal structure of the theory. In particular, data for the magnetic susceptibility indicated that the approach is indeed a viable numerical approach to the Kondo effect out of equilibrium.

Using the function theory of several complex variables, a framework for the analytic continuation of dynamic correlation functions was provided by means of an axiomatic characterization of the Green's function. In particular, kernel functions connecting real-time and imaginary-time structure of the theory were derived, and a one-to-one linear correspondence of real-time and imaginary-time quantities was established. Whereas the imaginary-time structure is characterized by holomorphic Green's function branches on wedges, the real-time structure is characterized by the edges of these wedges.

Employing the kernel functions, we were able to extend the standard MaxEnt approach for the ill-posed analytic continuation of effective-equilibrium quantum Monte-Carlo data to the non-equilibrium problem. Considering a single analytic wedge, equilibrium results for spectral functions were reproduced. However, it was found impossible to utilize a single-wedge approach for the non-equilibrium situation.

At this point, an asymptotically exact continuity constraint to the overall structure was employed. It enabled the inclusion of data from several distinct wedges to the MaxEnt Bayesian inference of the non-equilibrium spectral function. By this, the severity of the inverse problem was reduced. Well-controlled reproducible true nonequilibrium results were obtained for weak and intermediate correlation strengths. Derived $I-V$ curves were in good agreement with other methods. For the extension 
to strong correlations, an exact generalization of the formalism was suggested.

The generalization demands for an extension of the analytic continuation algorithm for dynamical quantities. It brings along a considerably large set of additional degrees of freedom which have to be inferred sufficiently accurately by the MaxEnt procedure. It is an open question to which extent information about the non-equilibrium strongcoupling regime may be inferred. In particular, it will have to be investigated how much prior information about the structure of edge functions is required in order to set up appropriate default models.

For this purpose, additional experience from the analysis of static quantities may play a key role, as they can be numerically accessed with much less difficulty. Again, the systematic construction of default models which contain more prior information and thus yield more reliable results seems promising. This requires a more extensive formal analysis of quantities such as high-energy tails of the effective-equilibrium observables $\langle\hat{A}\rangle\left(\mathrm{i} \varphi_{m}\right)$.

As far as the validity of the Matsubara-voltage formalism itself is concerned, our recent perturbative investigations [54] showed that for the chosen definition of the non-equilibrium state, the theory successfully maps to the corresponding SchwingerKeldysh real-time theory. However, the Kondo effect is a non-perturbative phenomenon. Furthermore, there do exist several different, possibly inequivalent, procedures to introduce the non-equilibrium ensemble. One can therefore at present not state with certainty that the theoretical framework is correct. The essential agreement of our strong-coupling magnetic susceptibility data with the conceptually rather different SNRG method gives a substantial amount of convidence that in fact, both methods are capable of describing the correct Kondo physics. In this context, is however puzzling that surprisingly, the three numerical methods real-time quantum Monte-Carlo (RT-QMC), SNRG, and Matsubara-voltage theory disagree on the double occupancy, i.e. the behaviour of charge fluctuations. While still a qualitative agreement with RT-QMC is obtained, the SNRG yields even qualitatively different predictions.

Besides the conceptual validity and the further technical improvement of numerical estimates, the extendability of the formalism to more general transport scenarios is a major point. It has been shown that for certain types and geometries of quantum dots, as well as certain observables, it is not possible to use the Matsubara-voltage idea, at least in the present form [53]. This may be traced back to some compulsory symmetries of operators [54]. It is an open question if these constraints may be overcome by a different setup of the formalism. Even if the answer is negative, progress within the present theory may gain some further valuable insights into the non-equilibrium Kondo effect in quantum dots, for example by including dissipative degrees of freedom or introducing additional sources of non-equilibrium such as temperature gradients. 


\section{A. Uniqueness of Continuation}

In the following, we would like to show that the continuation of Matsubara data $G\left(\mathrm{i} \varphi_{m}, \mathrm{i} \omega_{n}\right)$ to the multisheeted holomorphic function $G(\underline{z})$ is unique, i.e. we will prove assumption 3 in section 5.3, relation (5.14). We would like to derive the uniqueness using the assumptions 1, 2, and 3' from section 5.3. Since the proof will involve some elementary geometry, it will be accompanied by several sketches. It can be seen as a generalization of the Baym-Mermin result on the uniqueness of the analytic continuation of single-variable Matsubara Green's functions [98].

We may focus our attention to a single wedge $T^{C}$ which is defined by subsequent branch cuts from Eqs. (5.12). The data $G\left(\mathrm{i} \varphi_{m}, \mathrm{i} \omega_{n}\right)$ which are located in the wedge are our starting point, $\left(\varphi_{m}, \omega_{n}\right)^{T} \in C$. Without loss of generality we can assume that we have entire lines of data, $G\left(\mathrm{i} \varphi_{m}, \mathrm{i} \omega_{I}\right), \omega_{I} \in \mathbb{R}$, because arbitrary continuous imaginary $\omega_{I}$ may be computed by Fourier transform in the $\varphi_{m}$ th effective-equilibrium theory, having again $\left(\varphi_{m}, \omega_{I}\right)^{T} \in C$. Let us denote the effective-equilibrium data range by

$$
E_{0}:=\left\{\mathrm{i}\left(\varphi_{m}, \omega_{I}\right)^{T} \mid m \in \mathbb{Z}, \omega_{I} \in \mathbb{R}\right\} \cap T^{C} .
$$

These lines of known data of the unknown function $G(\underline{z})$ in the wedge $T^{C}$ are depicted in Fig. A.1. They constitute one-dimensional lines in the four-dimensional wedge $T^{C}$ for which the function $\left.G\right|_{T^{C}}$ shall be reconstructed.

We will, step by step, prove the uniqueness of the continuation of the imaginarytime data by applying biholomorphic maps and the identity theorem of complex analysis. The central idea will be to extend larger and larger subsets for which a

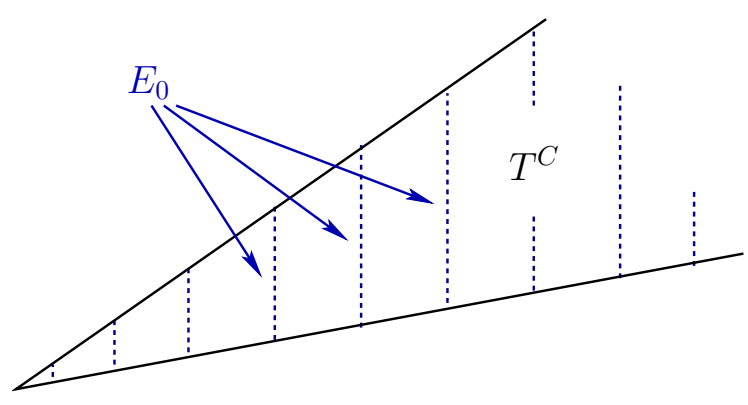

Figure A.1: The wedge to be considered. The dash-dotted lines denote the data yielded by imaginary-time theory. 


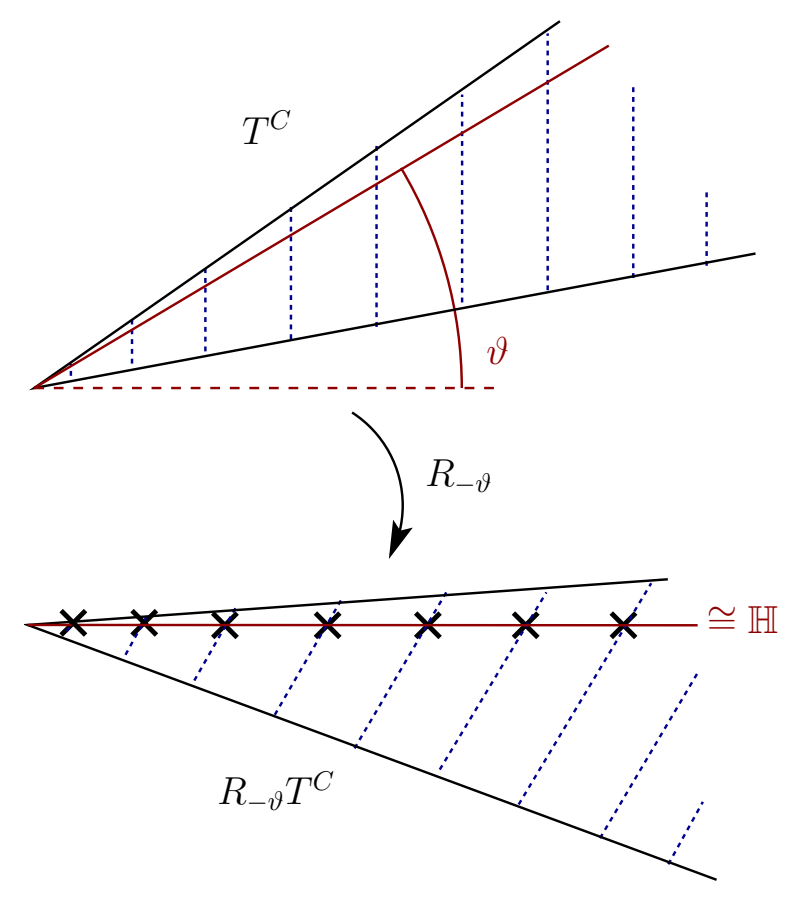

Figure A.2: A single line contained by the cone $C$ may after biholomorphic rotation $R_{-\vartheta}$ and subsequent complexification be interpreted as the upper half plane $\mathbb{H}$ of $\mathbb{C}$.

unique continuation is obtained.

\section{A.1. Reconstruction of edge values using complex lines which are isomorphic to $\mathbb{H}$}

Due to assumptions 1 and 2, we found that the Green's function $\left.G\right|_{T^{C}}$ may be reconstructed from their edge values, using Eqs. (5.41) and (5.42). Therefore, it suffices to show that we can reconstruct all edge values of the function $G$ from the data $\left.G\right|_{E_{0}}$.

We will first show that one may reconstruct a certain set of single lines through zero on the edge. Each of these lines is defined by an angle $\vartheta$. All function values on the line may be reconstructed if the angle $\vartheta$ is contained by the cone $C$.

The proof of the latter statement is the following. Consider a single line in the cone $C$, given by the angle $\vartheta$. A biholomorphic rotation in the sense of section 5.4.2 can then be applied in such a way that the line is horizontal and may after complexification be interpreted as the upper half plane $\mathbb{H}$ of $\mathbb{C}$, see Fig. A.2. The real line is then associated to a horizontal line on the edge of $R_{-\vartheta} T^{C}$.

The biholomorphic equivalent to the yet unknown function is now $\tilde{G}(\underline{z})=G\left(R_{-\vartheta}^{-1}\right.$. 
$\underline{z})$. Note that the line $(0, \mathrm{i} \lambda)^{T}$ in $R_{-\vartheta} T^{C}, \lambda>0$, contains infinitely many known values of $\tilde{G}(\underline{z})$. These are denoted by the crosses in Fig. A.2. Extending to the upper half plane $(0, \mathbb{H})$, one may apply the identity theorem of complex analysis for reconstructing $\tilde{G}(\underline{z})$ on the whole plane $(0, \mathbb{H})$. In particular, the boundary values $\tilde{G}\left(0, \mathbb{R}+\mathrm{i}^{+}\right)$are recovered. This proves the statement of this subsection.

\section{Identity Theorem}

Let us comment on the satisfaction of the assumptions of the identity theorem. Since $(0, \infty)$ is the accumulation point of the known data points on $(0, \mathbb{H})$, i.e. of the "series of crosses in Fig. A.2", given by $(0, \mathbb{H}) \cap R_{-\vartheta} E_{0}$, we have to show that $\tilde{G}(0,1 / z)$ may be extended to an analytic function at $z=0$. Once this is possible, the function is uniquely determined by the set of function values.

Combining assumptions 1 and 3', we know that $G\left(\zeta \underline{x}^{(0)}\right)$ (in the sense of assumption 3') behaves like a conventional Green's function, because the singular case coincides with a branch cut which is by construction not contained by the wedge. Due to this rapid decay one may extend $\tilde{G}$ to the lower half plane such that $\tilde{G}\left(0, z^{*}\right)=$ $\tilde{G}(0, z)^{*}$ and is holomorphic at $z=\infty$. This can be done explicitly using a spectral representation with respect to the boundary values of $\operatorname{Im} \tilde{G}(0, z)$ on the real axis. The spectral representation exists due to the $1 / z$ asymptotics which lets the line integral contribution vanish on the infinitely large semicircle attached to the real axis. Note that this construction is also compatible with the symmetry relation $G\left(-\mathrm{i} \varphi_{m},-\mathrm{i} \omega_{n}\right)=G\left(\mathrm{i} \varphi_{m}, \mathrm{i} \omega_{n}\right)^{*}$.

As a consequence, the identity theorem is applicable for $\tilde{G}(0, z)$ at $z=\infty$ such as it is for regular Matsubara Green's functions.

One may also think of $G\left(\zeta \underline{x}^{(0)}\right), \zeta \in \mathbb{H}$ as a meromorphic function of $\zeta \in \mathbb{C}$, because it may due to boundedness and $1 / \zeta$ asymptotics be approximated arbitrarily well by a meromorphic function, such as in an infinite Padé expansion. Since meromorphic functions on $\mathbb{C}$ are holomorphic on the Riemannian sphere, the identity theorem holds at the accumulation point $\infty$.

\section{Resulting reconstruction of edge values}

Sweeping through all possible angles $\vartheta$ which are contained by the cone $C$, the uniquely reconstructed edge behaviour of $\left.G\right|_{T^{C}}$ is given by the area depicted in Fig. A.3.

It is obviously given by

$$
\operatorname{Edge}_{T^{C}}^{(\text {recon, } 0)}:=C \cup(-C)
$$




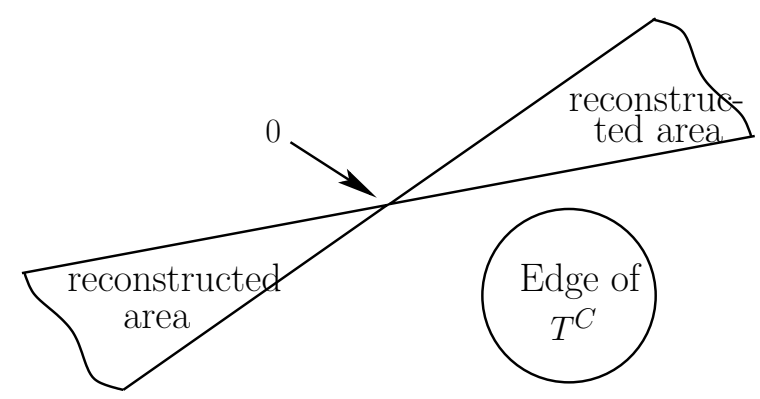

Figure A.3: Uniquely reconstructed range $\operatorname{Edge}_{T^{C}}^{(\text {recon,0) }}$ of $\left.G(\underline{z})\right|_{T^{C}}$ on the edge of $T^{C}$ following from the partial argument of subsection A.1. The wiggly lines in the boundary mean that the area extends to infinity.

\section{A.2. Extending the unique range to the entire edge}

In order to show that the function values of $\left.G\right|_{T^{C}}$ are also uniquely defined by $\left.G\right|_{E_{0}}$ for the complement of $\operatorname{Edge}_{T^{C}}^{(\text {recon,0) }}$, the argument has to be extended in a similar way. The trick is to consider yet another set of $\mathbb{H}$-isomorphic subspaces and then apply the argument of the last section to a larger set of data.

\section{Extending the known data range within the wedge}

The first step is depicted in Fig. A.4. In contrast to before, we consider a constant angle $\vartheta_{0}$ and various lines which start at different points on the boundary of the cone with the orientation $\vartheta_{0}$.

After biholomorphic rotation to the wedge $R_{-\vartheta_{0}} T^{C}$ we can again complexify the lines

$$
\tilde{l}_{\tilde{y}^{(0)}}:=\left\{\mathrm{i} \underline{\tilde{y}}^{(0)}+\mathrm{i}(0, \lambda)^{T}, \lambda>0\right\} ; \underline{\tilde{y}}^{(0)} \in \partial C
$$

to

$$
\tilde{L}_{\underline{\tilde{y}}^{(0)}}:=\mathrm{i} \underline{\tilde{y}}^{(0)}+\left(\begin{array}{c}
0 \\
\mathbb{H}
\end{array}\right)
$$

The isomorphy of $\tilde{L}_{\tilde{y}^{(0)}}$ to $\mathbb{H}$ and assumption 3' again enable us to apply the identity theorem to the crossed data in Fig. A.4, namely to the infinite sequence $\left.\left(G \circ R_{\vartheta}\right)\right|_{\left(R_{-\vartheta} E_{0}\right) \cap \tilde{L}_{\tilde{\tilde{y}}}(0)}$.

By this, the transformed Green's function $\tilde{G}=G \circ R_{\vartheta_{0}}$ is reconstructed for all points of the set

$$
D:=\bigcup_{\underline{\tilde{y}}^{(0)} \in \partial C} \tilde{L}_{\underline{\tilde{y}}^{(0)}}=\left(R_{-\vartheta_{0}} T^{C}\right) \cap\left(\begin{array}{c}
\mathrm{i} \mathbb{R} \\
\mathbb{C}
\end{array}\right) .
$$




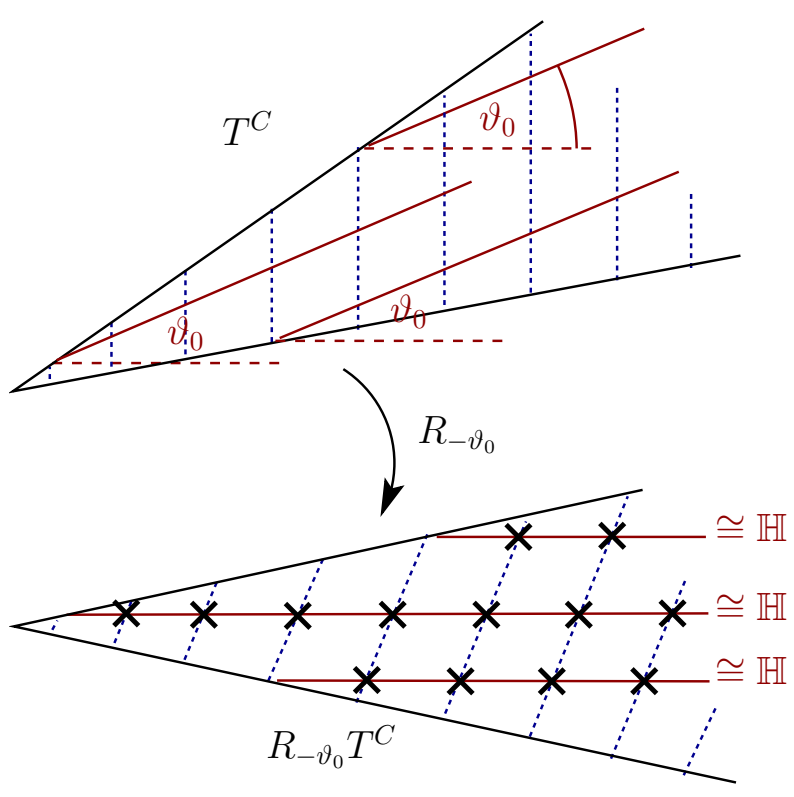

Figure A.4: Enhancing the formal holomorphic reconstruction within the wedge.

For simplicity, we may now just look at a subset of $D$, namely

$$
\tilde{E}_{1}:=R_{-\vartheta_{0}} E_{0}+\left(\begin{array}{c}
0 \\
\mathbb{R}
\end{array}\right) .
$$

It enables us to see that the values of $G$ are now known on the set

$$
E_{1}:=E_{0}+R_{\vartheta_{0}}\left(\begin{array}{c}
0 \\
\mathbb{R}
\end{array}\right) \text {. }
$$

\section{Full Reconstruction of the Edge}

With the information from $E_{1}$ one may reinterpret the procedure associated with Fig. A.2 and described in section A.1. The dashed lines of known data now contain an additional real dimension along the direction $R_{\vartheta_{0}}(0,1)^{T}$.

We can use each point $\lambda \cdot R_{\vartheta_{0}}(0,1)^{T}(\lambda \in \mathbb{R})$ of this new degree of freedom as an offset of the lines used in section (A.1) and reapply the entire procedure. Using the resulting affine subspaces, the Green's function may be reconstructed on further regions of the edge which are affine to the one in Fig. A.3, namely

$$
\begin{aligned}
\operatorname{Edge}_{T^{C}}^{(\text {recon }, \lambda)}:= & \operatorname{Edge}_{T^{C}}^{(\text {recon }, 0)} \\
& +\lambda \cdot R_{\vartheta_{0}}(0,1)^{T} .
\end{aligned}
$$




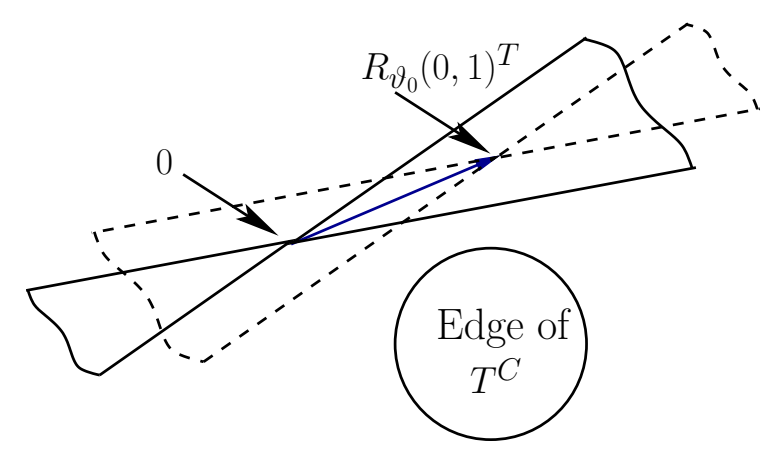

Figure A.5: Reconstructing the Green's function on the complete edge. The reconstructed area Edge $_{T C}^{(\text {recon,0) }}$ (see Fig. A.3 and Eq. (A.2)) may be extended by affine transformations along the $R_{\vartheta_{0}}(0,1)^{T}$ direction using the information from the set $E_{1}$.

Applying the argument to all $\lambda \in \mathbb{R}$ reconstructs the entire edge and hence the entire Green's function $\left.G\right|_{T^{C}}$, because $\bigcup_{\lambda \in \mathbb{R}} \operatorname{Edge}_{T^{C}}^{(\text {recon, }, \lambda)}=\operatorname{Edge}_{T^{C}}$.

This "affine procedure" is sketched in Fig. A.5. 


\section{B. A-priori information about residual discontinuities}

In the present appendix, we will study what can be learned a priori about the structure of terms which violate the continuity assumption which is fundamental to the approach lined out in chapter 7 . It is insightful to study how the resulting difference of two given edge values is structured, namely to investigate the branch-point discontinuity

$$
R^{\left(\vartheta, \vartheta^{\prime}\right)}(\underline{x}):=\operatorname{Re} G\left(\underline{x}+\mathrm{i} 0^{\vartheta}\right)-\operatorname{Re} G\left(\underline{x}+\mathrm{i} 0^{\vartheta^{\prime}}\right)
$$

for arbitrary values $\vartheta, \vartheta^{\prime} \in[0,2 \pi)$ as a function of $\underline{x} \in \mathbb{R}^{2}$.

\section{Angular structure}

Obviously, due to Eq. (7.6) we have

$$
R^{(\vartheta, \vartheta)} \equiv R^{(\vartheta, \vartheta+\pi)} \equiv 0
$$

for all $\vartheta . R^{(\vartheta, \vartheta)} \equiv 0$ if $\vartheta$ and $\vartheta^{\prime}(+\pi)$ belong to the same wedge.

\section{Structure due to continuity of imaginary-time data}

Using the nontrivial empirical fact that the continuous-time QMC data $\Sigma\left(\mathrm{i} \varphi_{m}, \mathrm{i} \omega_{n}\right)$ are continuous as a function of $\varphi_{m}$ and $\omega_{n}$, we can derive certain relations for $R^{\left(\vartheta, \vartheta^{\prime}\right)}$.

The empirical observation that the quantum Monte Carlo data are continuous as a function of $i \omega_{n}$, as one crosses higher-order branch cuts, yields the following structure. Let $\vartheta_{0}$ be the orientation of the corresponding branch cut. Then, due to the observed continuity, we have

$$
\begin{gathered}
G\left(\mathrm{i} \rho \sin \left(\vartheta_{0}-\delta\right), \mathrm{i} \rho \cos \left(\vartheta_{0}-\delta\right)\right)= \\
=G\left(\mathrm{i} \rho \sin \left(\vartheta_{0}+\delta\right), \mathrm{i} \rho \cos \left(\vartheta_{0}+\delta\right)\right),
\end{gathered}
$$

for any $\rho>0$.

Using the identity theorem along the directions $\vartheta_{0} \pm \delta$, one find that the relation

$$
R^{\left(\vartheta_{0}-\delta, \vartheta_{0}+\delta\right)}\left(\rho \sin \left(\vartheta_{0}\right), \rho \cos \left(\vartheta_{0}\right)\right)=0
$$

holds for any $\rho>0$. Consequently, the continuity in Matsubara space induces a 
continuity relation in the edge space. Similarly, one could construct relations for the derivatives of $G$ which help constrain $R^{\left(\vartheta, \vartheta^{\prime}\right)}(\underline{x})$.

\section{High-energy structure}

Let us also consider the high-energy limit

$$
|\underline{x}| \gg \max \left\{\Gamma,|U|,|\Phi|,\left|\varepsilon_{d}\right|\right\} .
$$

For this, $\left|G\left(\underline{x}+\mathrm{i} 0^{\vartheta}\right)\right|$ is only significantly greater than zero if $x_{\omega} \approx \pm x_{\varphi} / 2$, according to point 3', section 5.4.2. The "+" and "-" cases imply a separation of energy scales. ${ }^{1}$ For both of these two energy scales, the closedness under multiplication is recovered.

One can easily see the recovery of the multiplicative structure in the high-energy limit by investigating the bare Green's function (5.52). The "+" and "-" energy scales are given by the $\alpha=-$ and $\alpha=+$ addends (5.53) in Eq. (5.52), respectively. Each of the addends satisfies the multiplication rule, because the absolute value of their imaginary part remains the same for all $\vartheta$ at fixed $\underline{x}$. Same is true for the sum of the corresponding $\sum_{i} \alpha_{i}=\mp 1$ addendends in the second-order self-energy (5.16).

Therefore, we conclude that

$$
\lim _{\underline{x \rightarrow \infty}} R^{(\vartheta, \vartheta)}=0,
$$

in contrast to the infinite- $\underline{x}$ limiting behaviour for $G$ itself.

\section{Consequences for the analytic structure}

Although the assumption

$$
\operatorname{Re} G\left(\underline{x}+\mathrm{i} 0^{\vartheta}\right)=\operatorname{Re} G\left(\underline{x}+\mathrm{i} 0^{\vartheta^{\prime}}\right),
$$

for all $\vartheta, \vartheta^{\prime}$, is apparently incorrect, the errors $R^{\left(\vartheta, \vartheta^{\prime}\right)}(\underline{x})$ are localized in $\underline{x}$-space.

In other words: The relation gives a correct picture of how the wedges are correlated in the higher-energy range. When assuming the relation, additional low-energy degrees of freedom have to be introduced in order to reobtain an exact continuation theory.

\section{Dyson's equation}

The missing closedness under multiplication can a priori be expected to be particularly problematic along the singular directions of the bare Green's function. It will

\footnotetext{
${ }^{1}$ The two separated branches nevertheless appear to be "interacting" with each other, due to the absence of a conservation law for the $Y_{0}$ quantum number in Coulomb scattering processes (section 3.4.1), the relevance of which is particularly evident for $\left|x_{\varphi}-\Phi\right| \gg \Gamma$.
} 
become clear in an a-posteriori analysis that therefore data which do not cross the bare Green's functions branch cut are valuable for an analytic continuation based on the assumption that the real part is continuous (cf. section 7.3). 


\section{Commutator relations of $\mathcal{Q}_{\vartheta}^{\text {(edge) }}$}

In the following we will derive the commutator relations (7.14), (7.15), and (7.16) for the edge-to-edge map $\mathcal{Q}_{\vartheta}^{\text {(edge) }}$.

\section{C.1. Translational invariance}

Let us consider the action of $\mathcal{Q}_{\vartheta}^{(\text {edge })}=\mathcal{R}_{\vartheta} \mathcal{H R}_{\vartheta}^{-1} \mathcal{H}$ on a function $\tilde{A}(x, y)$ which is translated by the operator $\mathcal{T}_{\underline{X}}$, where we set $\underline{X}=(X, Y)^{T}$. We also write $\underline{x}:=(x, y)^{T}$. As a first step, we apply the Hilbert transform,

$$
\begin{aligned}
\left(\mathcal{H}_{\mathcal{T}_{\underline{X}}} \tilde{A}\right)(\underline{x}) & =\frac{1}{\pi} \oint \mathrm{d} \tilde{y} \frac{\tilde{A}(x-X, \tilde{y}-Y)}{y-\tilde{y}} \\
& =\frac{1}{\pi} \oint \mathrm{d} \tilde{y} \frac{\tilde{A}(x-X, \tilde{y})}{(y-Y)-\tilde{y}} .
\end{aligned}
$$

Using the short-hand notation $c:=\cos \vartheta, s:=\sin \vartheta$, we then have

$$
\left(\mathcal{R}_{\vartheta}^{-1} \mathcal{H} \mathcal{T}_{\underline{X}} \tilde{A}\right)(\underline{x})=\frac{1}{\pi} \not \mathrm{d} \tilde{y} \frac{\tilde{A}(c x+s y-X, \tilde{y})}{(-s x+c y-Y)-\tilde{y}} .
$$

In the next two steps one obtains

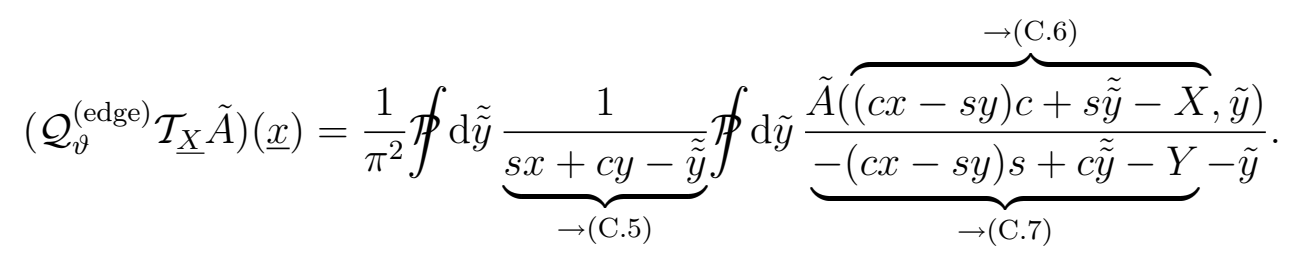

By substituting $\underline{X}=0$ and then multiplying with $\mathcal{T}_{\underline{X}}$ from the left, one finds

$$
\begin{aligned}
&\left(\mathcal{T}_{\underline{X}} \mathcal{Q}_{\vartheta}^{(\text {edge })} \tilde{A}\right)(\underline{x})=\frac{1}{\pi^{2}} \not \mathrm{d} \tilde{\tilde{y}} \frac{1}{s(x-X)+c(y-Y)-\tilde{\tilde{y}}} \cdot \\
& \cdot \not \oint \mathrm{d} \tilde{y} \frac{\tilde{A}((c(x-X)-s(y-Y)) c+s \tilde{\tilde{y}}, \tilde{y})}{-(c(x-X)-s(y-Y)) s+c \tilde{\tilde{y}}-\tilde{y}} .
\end{aligned}
$$

In order to verify that the expressions (C.3) and (C.4) are, in fact, equal, we consider 
the following system of linear equations:

$$
\begin{aligned}
s x+c y-\tilde{\tilde{y}} & =s x_{0}+c y_{0}-y^{*} \\
(c x-s y) c+s \tilde{\tilde{y}}-X & =\left(c x_{0}-s y_{0}\right) c+s y^{*} \\
-(c x-s y) s+c \tilde{\tilde{y}}-Y & =-\left(c x_{0}-s y_{0}\right) s+y^{*} c
\end{aligned}
$$

in matrix form:

$$
\underbrace{\left(\begin{array}{ccc}
-1 & s & c \\
s & c^{2} & -s c \\
c & -s c & s^{2}
\end{array}\right)}_{=: M} \cdot\left(\begin{array}{l}
\tilde{\tilde{y}} \\
x \\
y
\end{array}\right)-\left(\begin{array}{l}
0 \\
X \\
Y
\end{array}\right)=\underbrace{\left(\begin{array}{ccc}
-1 & s & c \\
s & c^{2} & -s c \\
c & -s c & s^{2}
\end{array}\right)}_{=: M} \cdot\left(\begin{array}{l}
y^{*} \\
x_{0} \\
y_{0}
\end{array}\right) .
$$

The equations correspond to the idea of substituting the terms in equation (C.3) as denoted there in such a way that $y^{*}$ is the new integration variable, $\int \mathrm{d} \tilde{\tilde{y}} \rightarrow \kappa \int \mathrm{d} y^{*}$, where $\kappa$ is some regular prefactor from the integral transformation, and such that the external variables $x$ and $y$ are replaced by $x_{0}$ and $y_{0}$. We will see that the resulting form will exactly be (C.4):

The system (C.5)-(C.7) can be solved by inversion of the matrix $M$ : One has

$$
M^{-1}=\left(\begin{array}{ccc}
0 & s & c \\
s & 1 & 0 \\
c & 0 & 1
\end{array}\right),
$$

$\operatorname{det} M=-1$, and consequently the well-defined solution

$$
\begin{aligned}
& y^{*}=\tilde{\tilde{y}}-(s X+c Y), \\
& x_{0}=x-X, \\
& y_{0}=y-Y .
\end{aligned}
$$

The integral transformation constant $\kappa=1$, and performing the substitutions (C.5)(C.7) in (C.3) yields (C.4), when $y^{*}$ is again renamed as $\tilde{\tilde{y}}$.

Therefore, $\left[\mathcal{Q}_{\vartheta}^{\text {(edge) }}, \mathcal{T}_{\underline{X}}\right]=0$. 


\section{C.2. Scale invariance}

Similarly, we show the scale invariance (7.15). We have

$$
\begin{aligned}
& \left(\mathcal{Q}_{\vartheta}^{\text {(edge) }} \Lambda_{\lambda} \tilde{A}\right)(\underline{x})=\frac{\lambda^{2}}{\pi^{2}} \oint \mathrm{d} \tilde{\tilde{y}} \frac{1}{s x+c y-\tilde{\tilde{y}}} \oint \mathrm{d} \tilde{y} \frac{\tilde{A}(\lambda[(c x-s y) c+s \tilde{\tilde{y}}], \lambda \tilde{y})}{-(c x-s y) s+c \tilde{\tilde{y}}-\tilde{y}}( \\
& =\frac{1}{\pi^{2}} \not \mathrm{d} \tilde{\tilde{y}} \frac{1}{s x+c y-\tilde{\tilde{y}} / \lambda} \text {. } \\
& \cdot \oint \mathrm{d} \tilde{y} \frac{\tilde{A}((c \lambda x-s \lambda y) c+s \tilde{\tilde{y}}, \tilde{y})}{-(c x-s y) s+c \tilde{\tilde{y}} / \lambda-\tilde{y} / \lambda} \\
& =\frac{\lambda^{2}}{\pi^{2}} \not \mathrm{d} \tilde{\tilde{y}} \frac{1}{s \lambda x+c \lambda y-\tilde{\tilde{y}}} \text {. } \\
& \oint \mathrm{d} \tilde{y} \frac{\tilde{A}((c \lambda x-s \lambda y) c+s \tilde{\tilde{y}}, \tilde{y})}{-(c \lambda x-s \lambda y) s+c \tilde{\tilde{y}}-\tilde{y}} \\
& =\left(\Lambda_{\lambda} \mathcal{Q}_{\vartheta}^{(\text {edge })} \tilde{A}\right)(\underline{x}) \text {. }
\end{aligned}
$$

\section{C.3. Absence of rotational invariance}

We provide a simple example for which $\left[\mathcal{Q}_{\vartheta}^{(\text {edge })}, \mathcal{R}_{\vartheta^{\prime}}\right] \neq 0$.

We consider the bare $\tilde{A}_{0}$, equation (5.59). Setting $\vartheta=\vartheta^{\prime}=\pi / 2$, we find

$$
\begin{aligned}
\left(\mathcal{Q}_{\pi / 2}^{(\text {edge })} \tilde{A}_{0}\right)(\underline{x}) & =\sum_{\alpha= \pm 1} \frac{\alpha \Gamma_{\alpha} / \pi}{\left(x_{\omega}-\alpha\left(x_{\varphi}-\Phi\right) / 2-\varepsilon_{d}\right)^{2}+\Gamma^{2}}, \text { and } \\
\left(\mathcal{R}_{\pi / 2} \mathcal{Q}_{\pi / 2}^{(\text {edge) }} \tilde{A}_{0}\right)(\underline{x}) & =\sum_{\alpha= \pm 1} \frac{\alpha \Gamma_{\alpha} / \pi}{\left(x_{\varphi}-\alpha\left(-x_{\omega}-\Phi\right) / 2-\varepsilon_{d}\right)^{2}+\Gamma^{2}} .
\end{aligned}
$$

On the other hand,

$$
\begin{aligned}
\left(\mathcal{R}_{\pi / 2} \tilde{A}_{0}\right)(\underline{x}) & =\sum_{\alpha= \pm 1} \frac{\Gamma_{\alpha} / \pi}{\left(x_{\varphi}-\alpha\left(-x_{\omega}-\Phi\right) / 2-\varepsilon_{d}\right)^{2}+\Gamma^{2}} \text {, and } \\
\left(\mathcal{Q}_{\pi / 2}^{(\text {edge })} \mathcal{R}_{\pi / 2} \tilde{A}_{0}\right)(\underline{x}) & =\sum_{\alpha= \pm 1} \frac{-\alpha \Gamma_{\alpha} / \pi}{\left(x_{\varphi}-\alpha\left(-x_{\omega}-\Phi\right) / 2-\varepsilon_{d}\right)^{2}+\Gamma^{2}} .
\end{aligned}
$$

Hence, $\mathcal{Q}_{\pi / 2}^{(\text {edge })} \mathcal{R}_{\pi / 2} \tilde{A}_{0}=-\mathcal{R}_{\pi / 2} \mathcal{Q}_{\pi / 2}^{(\text {edge })} \tilde{A}_{0}$, and therefore $\left[\mathcal{Q}_{\pi / 2}^{\text {(edge) }}, \mathcal{R}_{\pi / 2}\right] \neq 0$. 
C. Commutator relations of $\mathcal{Q}_{\vartheta}^{\text {(edge) }}$ 


\section{MaxEnt implementation for data from multiple wedges}

In this appendix chapter, the implementation of the MaxEnt algorithm for the $\mathcal{Q}$ mapping is described. Details on the computation of a numerical representation of $\mathcal{Q}$ are provided in appendix E. As pointed out there, the local test function width $\delta_{\underline{x}}$ for the map $\mathcal{Q}_{r, \vartheta}$ can be adjusted to the local grid resolution when the function $\tilde{A}(\underline{x})$ is discretized. The inverse problem for the inference of spectral properties using assumption (B.7) is, by construction,

$$
\operatorname{Im} G\left(\mathrm{i} \varphi_{m}, \mathrm{i} \omega_{n}\right)=\left(\mathcal{Q}_{r, \vartheta} \tilde{A}\right)\left(\mathrm{i} \varphi_{m}, \mathrm{i} \omega_{n}\right) .
$$

The values $r, \vartheta$ are those which specify the $T^{C_{r, \vartheta}}$ branch of $G$ the vector $\left(\mathrm{i} \varphi_{m}, \mathrm{i} \omega_{n}\right)^{T}$ is located in, as defined by point 1 of section 5.3. The spectral function of the dot electrons can then again be gained by evaluating the fiber

$$
A(\omega)=\tilde{A}(\Phi, \omega)
$$

of the inferred $(\vartheta=0)$-edge function, see section 6.2 .

\section{Discretization of $\tilde{A}\left(x_{\varphi}, x_{\omega}\right)$}

In contrast to the simpler approach depicted in section 6.2.1, the discretization of the edge function has to pay tribute to the strong interrelation between edge structure and branch cut structure which is revealed by $\mathcal{Q}^{(\text {edge) }}$, as described in section 7.2.3.

Especially, it turns out that the limiting behaviour along the singular directions of $\tilde{A}$ has to be captured numerically. In our experience, also the lateral structure of $\tilde{A}(\underline{x})$ along the singular directions has to be resolved, in order to improve numerical instabilities. We constructed a grid as follows. Let $\tilde{x}_{1}^{(i)}$ and $\tilde{x}_{2}^{(j)}$ be two variables which are discretized on $i$-th and $j$-th logarithmic grid points around zero, respectively. Then the grid $\underline{x}^{(i, j)}=\frac{\sqrt{5}}{20}\left(\begin{array}{cc}8 & 10 \\ -4 & 5\end{array}\right) \underline{\tilde{x}}^{(i, j)}$ yields an appropriate discretization of the edge, because the given matrix maps the double-cone $\mathbb{R}^{+} \times \mathbb{R}^{+} \cup \mathbb{R}^{-} \times \mathbb{R}^{-}$and its complement to the wedges defined by the singular directions. The test function width $\delta_{\underline{x}}$ can then be adjusted to the local grid resolution.

Also the high-energy structure of the Green's function $G$ has to be taken into ac- 
count explicitly, because along the singular directions, $G$ does not decay. In practice, it seems to be important to have a very large logarithmically discretized fit region, for which a $x_{\varphi} / \Gamma$ region of at most $[-800,800]$ is subject to modifications by the MaxEnt algorithm and a $x_{\omega} / \Gamma$ region of at most $[-400,400]$. The singular-direction contributions beyond this range also prove not to be negligible, in a test with the bare Green's function (see also the $G_{0}$ benchmark below). In order to take them into account, their contribution up to very large energies $\left(x_{\varphi} \approx 10^{5} \Gamma\right)$ is computed assuming a $G_{0}$-like structure along the directions, positioning adequately weighted $\delta$-spikes along it and substracting the corresponding contributions from the raw data, as done for the negative-spectral-function contributions of static observables in section 6.1.

\section{Kernel structure}

One has to keep in mind that the kernel $\mathcal{Q}_{r, \vartheta}$ may show rather sharp structures also in regions where the to-be-determined $\tilde{A}$ is expected to be very smooth and the MaxEnt discretization grid is consequently chosen to be rather coarse-grained. These potentially disturbing structures can already be seen from the plots of $\mathcal{P}_{r, \vartheta}$, which are displayed in Figs. 6.10 and 6.11. The convolution with the (transformed) test functions $\mathcal{Q}_{\vartheta}^{(\text {edge) }} f_{\underline{X}, \delta}$ is in general no cure for this problem, because $\mathcal{Q}_{\vartheta}^{(\text {edge) }} f_{\underline{X}, \delta}$ is even more sharply structured, on the scale $\delta$, which is of the order of the discretiziation scale (see figure 7.4).

In order to discuss this in more detail, some matrix elements of $\mathcal{Q}_{r, \vartheta}$ are plotted in figure D.1. Again, as compared to the $\mathcal{P}_{r, \vartheta}$ in Figs. 6.10 and 6.11, the orientation of the considered data point in Matsubara space defines the orientation of the structure which emerges in the kernel with respect to the $\left(x_{\varphi}, x_{\omega}\right)$ coordinates of $\tilde{A}$-space. A qualitative difference to figures 6.10 and 6.11 is the occurance of negative regions. These are generated by $\mathcal{Q}_{\vartheta}^{\text {(edge) }}$ in order to compute $\tilde{A}$ from the edge function of the wedge being considered due to the position of the $\left(\mathrm{i} \varphi_{m}, \mathrm{i} \omega_{n}\right)$ point. The negative and positive regions spread over a comparably wide range and will compete in the process of Bayesian inference, in which several $\left(\mathrm{i} \varphi_{m}, \mathrm{i} \omega_{n}\right)$ pairs and differently overlapping combinations of positive/negative regions are involved. The wide range of the regions appears to emerge from superimposing the $1 / x$ tails of $\mathcal{Q}_{\vartheta}^{(\text {edge })} f_{\underline{X}, \delta}$ which are dominant for $\vartheta \approx \pi / 2$ and $\vartheta \approx 3 \pi / 2$ and absent for $\vartheta \approx 0$, as well as $\vartheta \approx \pi$. Note that since the real-part assumption (B.7) becomes exact for larger energies, this feature is expected to be contained in the kernel of an optimal continuation theory of Green's functions within the Matsubara voltage formalism. The kernel structure indicates that due to the leverage of the single-wedge constraint (section 6.2.2), the nonequilibrium spectral function could now well be resolved. In the following, the interacting branch cut geometry will always be used for the operator $\mathcal{Q}_{r, \vartheta}$. For brevity, the accordingly defined operator will be shortly written as $\mathcal{Q}$. 


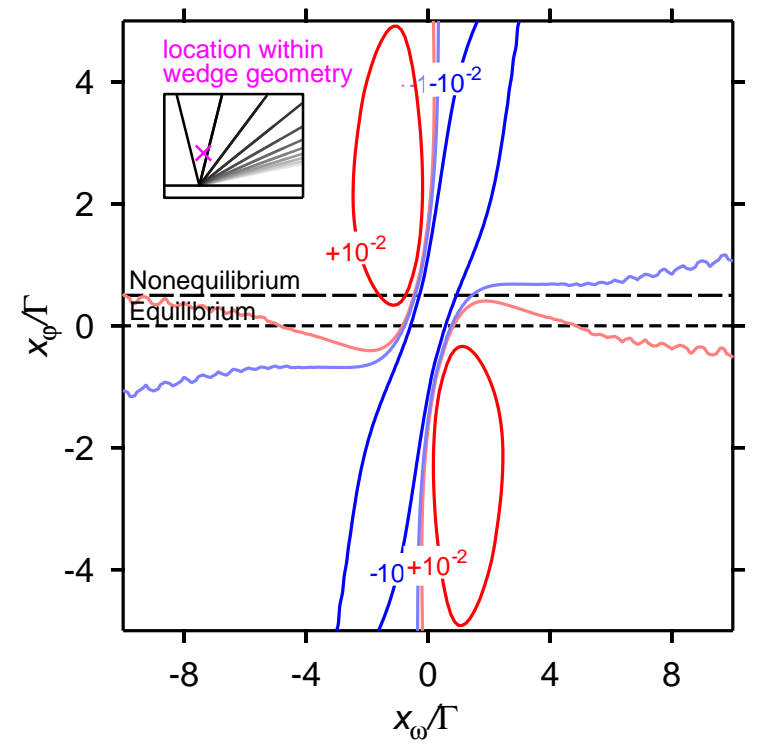

(a) $m=1, n=0(\beta \Gamma=5.0)$

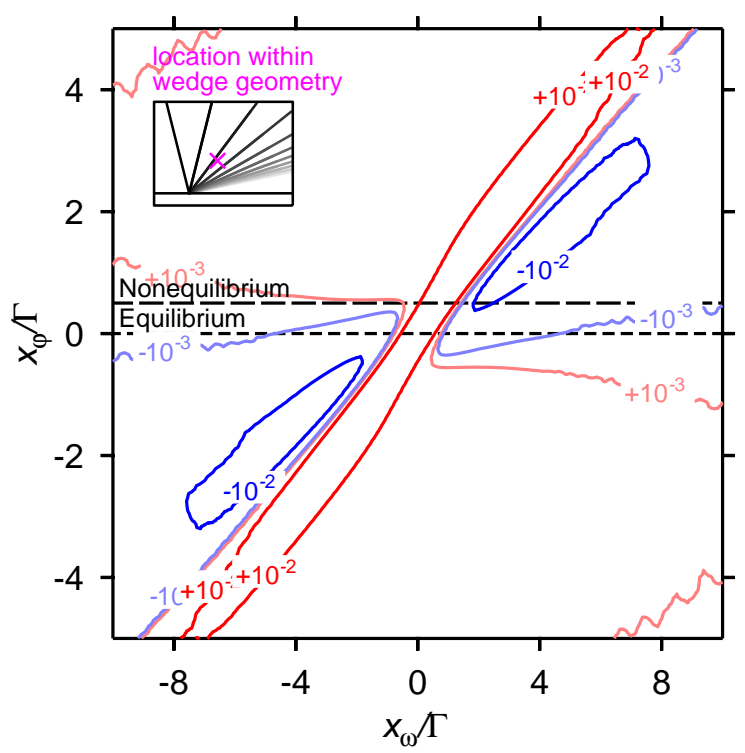

(c) $m=1, n=3(\beta \Gamma=5.0)$

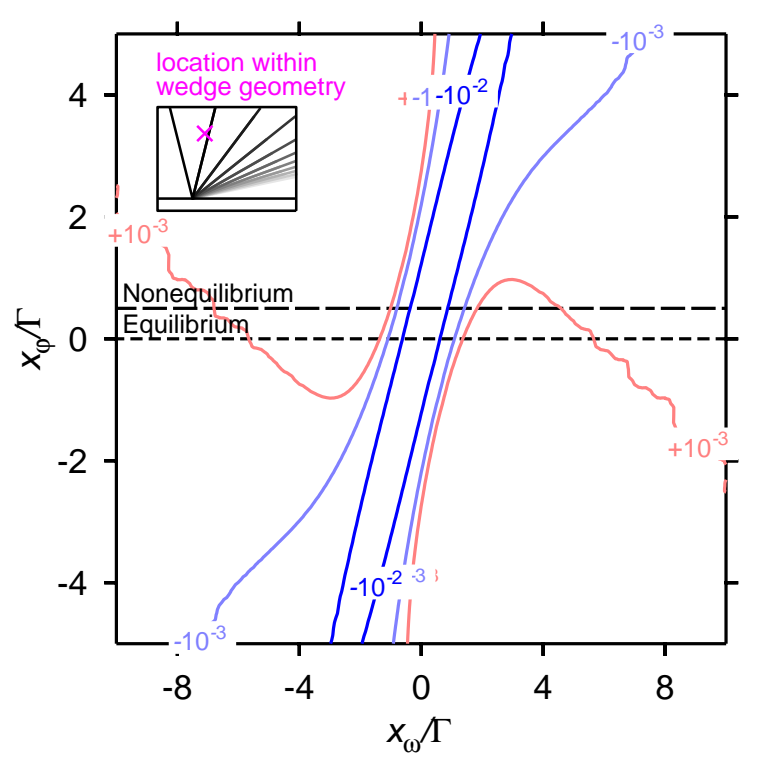

(b) $m=2, n=1(\beta \Gamma=5.0)$

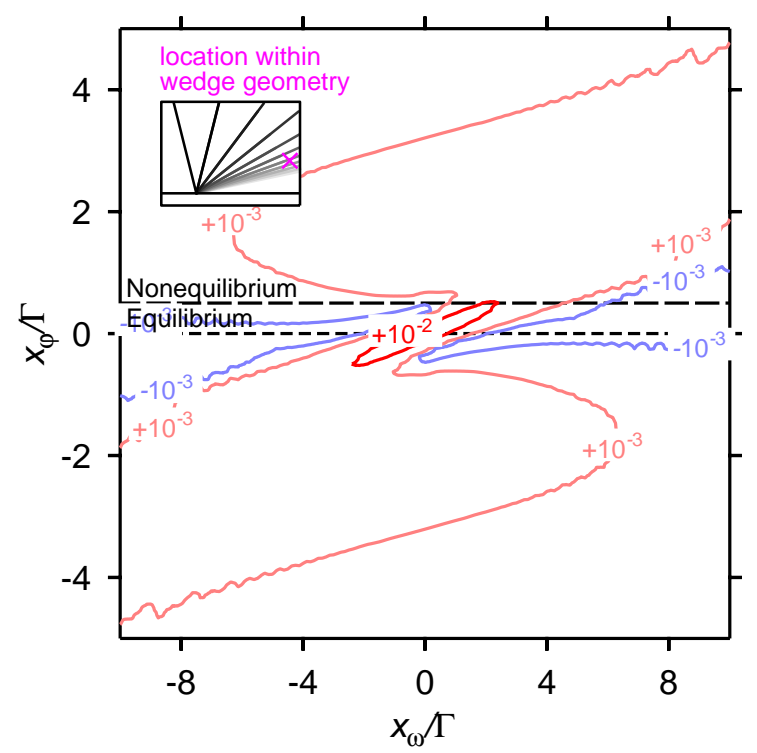

(d) $m=1, n=11(\beta \Gamma=5.0)$

Figure D.1: Cut through $\mathcal{Q}_{r, \vartheta}$ in $\tilde{A}$-space for different pairs of $\mathrm{i} \varphi_{m}$ and $\mathrm{i} \omega_{n}$, at $\beta \Gamma=5.0$. The wedge opening ratios $r$ and wedge orientations $\vartheta$ (figure 5.4) are chosen according to the interacting branch-cut geometry (chapter 3.4.2). The "nonequilibrium" line represents the location of the dot-electron spectral function for a system with source-drain voltage $e \Phi=0.5 \Gamma$. The location maps for Matsubara data are on the same scale as the ones in figures 6.10 and 6.11 . Wiggly structures at higher energies result from the increasingly coarse-grained $\tilde{A}$ grid. 


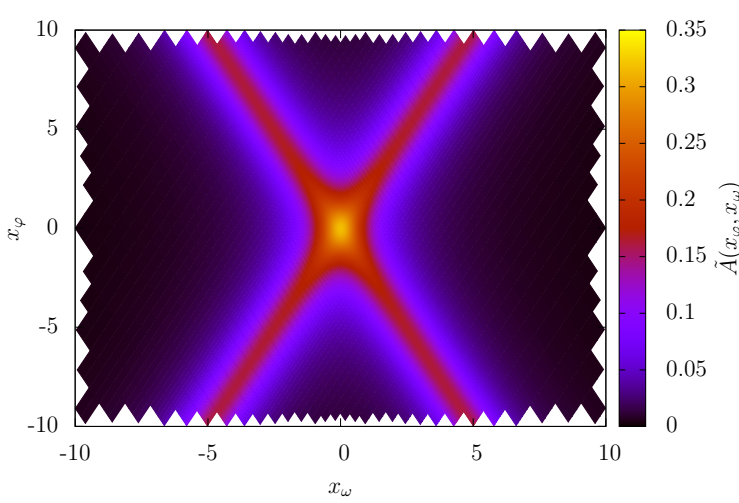

(a) exact $\tilde{A}_{0}\left(x_{\varphi}, x_{\omega}\right)$

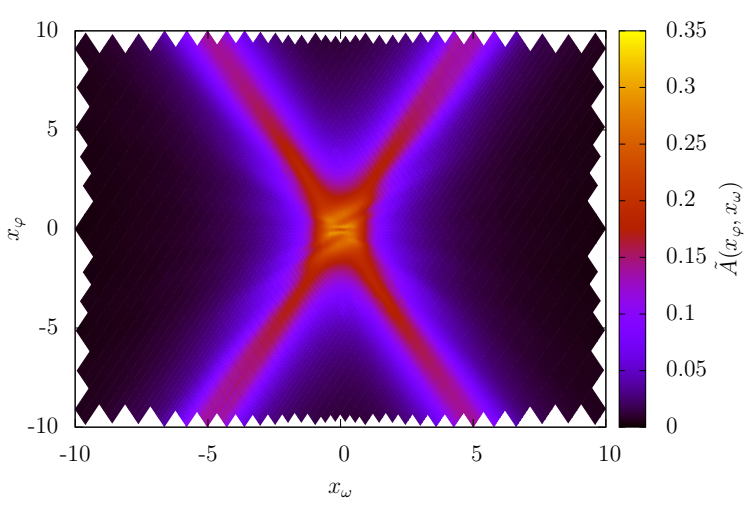

(c) $4 \times 4$ averaging

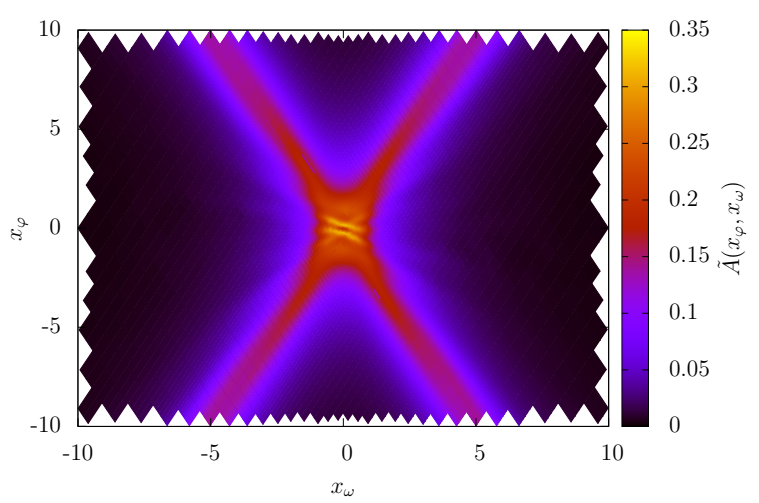

(b) $2 \times 2$ averaging

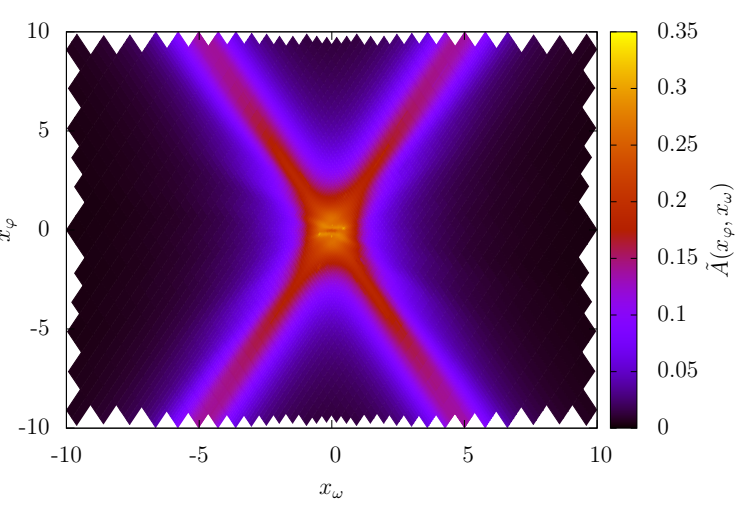

(d) $8 \times 8$ averaging

Figure D.2: Successive improvement of kernel quality by averaging out the local kernel structure within the local $\tilde{A}$ grid resolution. Data are shown for $U=0, \Phi=0, \beta \Gamma=5, n=$ $0, \ldots, 9, m=-3, \ldots, 3$ and a realistic mock diagonal covariance matrix $C=\operatorname{diag}\left(\frac{10^{-13}}{\Gamma^{2}}\right)$.

\section{Non-interacting Green's function as benchmark}

The fundamental assumption of this chapter, equation (B.7), is exact for $G_{0}$ (equation (5.57)). As a consequence, we use the noninteracting Green's function as a benchmark for our multiple-wedge numerical analytic continuation procedure, already assuming the interacting branch cut geometry for the construction of $\mathcal{Q}_{r, \vartheta}$. From a numerical point of view, the method is comprised of two technically challenging consecutive steps: First, the kernel and its high-energy convolution with the Green's function have to be evaluated numerically up to a certain precision. Second, an appropriate default model has to be defined and the MaxEnt has to converge to a good estimate in a controlled way.

In order to test the performance of the first step, we can take the exact solution as default model and run the MaxEnt with the discretized kernel. Due to the design of 


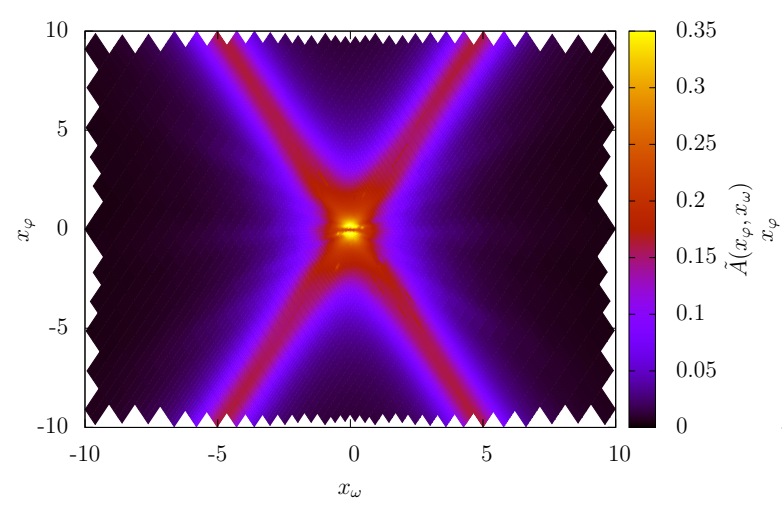

(a) $\sigma_{\text {def }}=1.2 \Gamma$

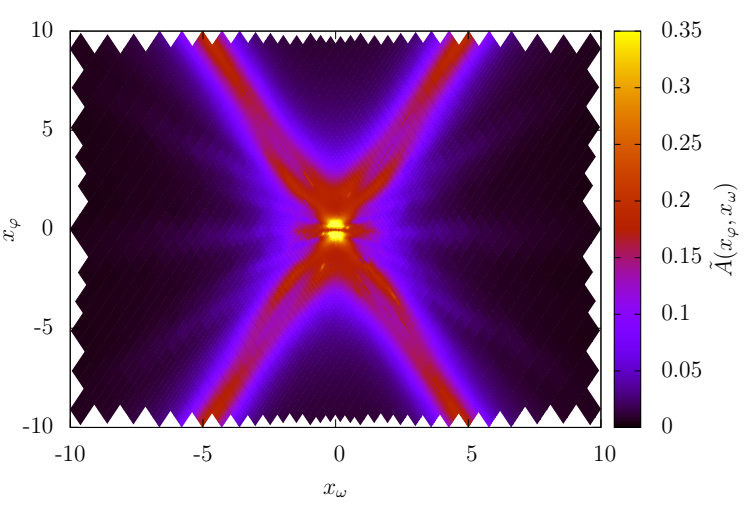

(b) $\sigma_{\text {def }}=1.5 \Gamma$

Figure D.3: Sensitivity to the default model, using the same parameters as in figure D.2d.

Bryan's algorithm [118], MaxEnt changes of the $\tilde{A}\left(x_{\varphi}, x_{\omega}\right)$ function will directly correspond to the numerical errors in the computation of the kernel matrix elements: evidence for changes of the exact solution is taken from the exact data due to numerical imperfections in the kernel. Without averaging out the sharp structures of the kernel for these regions, serious artifacts are obtained even for larger values of $\delta_{\underline{x}}$. This can be seen in the historic MaxEnt data shown figure D.2. Here, $\delta_{\underline{x}}$ is chosen adaptively with respect to the local kernel resolution, namely $\delta_{\underline{x}}=0.3 \cdot$ (local kernel grid resolution). The MaxEnt is able to modify the $\tilde{A}$ function on a large grid varying over the ranges $x_{\varphi} \in[-800,800], x_{\omega} \in[-400,400]$. As the local kernel resolution is increased, averaging out its structure within the $\tilde{A}$ grid, an increasingly appropriate discretization of the kernel is obtained. In the computations shown in figure D.2 realistic covariance weights for the imaginary-time data were assumed. If numerical errors $\gtrsim \sqrt{10^{-13}}$ were included into the realization of the kernel, there would probably be stronger deviations from $\tilde{A}_{0}\left(x_{\varphi}, x_{\omega}\right)$ than observed. For some single points the $\delta_{\underline{x}}$ is so small that the adaptive quadrature of the fourth integral in $\mathcal{Q}$ does not converge. This can be seen best in figure D.2d, because here the kernel discretization grid is eight times finer than the $\widetilde{A}\left(x_{\varphi}, x_{\omega}\right)$ discretization grid. Similarly, in the nonequilibrium situation, $\Phi \neq 0$, the function $\tilde{A}_{0}$ is not significantly altered by the $8 \times 8$-averaging kernel. This was tested explicitly also for large bias voltages, such as $e \Phi=\Gamma$.

The performance of the second step can be tested by using a kernel realization which succeeded in the first step and then performing runs with a modified default model. Because the noninteracting $\tilde{A}_{0}\left(x_{\varphi}, x_{\omega}\right)$ function has the correct singular behaviour as $\underline{x} \rightarrow \infty$ we investigate the dependence of the MaxEnt results on the following default models:

$$
\tilde{D}_{\sigma_{\mathrm{def}}}\left(x_{\varphi}, x_{\omega}\right)=\frac{1}{2 \pi} \sum_{\alpha= \pm 1} \frac{\sigma_{\mathrm{def}}}{\left(x_{\omega}-\frac{\alpha}{2}\left(x_{\varphi}-\Phi\right)\right)^{2}+\sigma_{\mathrm{def}}^{2}} .
$$


As compared to $\tilde{A}_{0}\left(x_{\varphi}, x_{\omega}\right)$, the width of the Lorentzians is varied. Using the bestquality kernel, i.e. $8 \times 8$-averaging (see figure D.2d), increasing the default-model width quickly results in spurious features (see figure D.4) in the low- to intermediateenergy region, even though $\mathcal{Q}$ represents an exact relation between data and $\tilde{A}$ and the numerical representation of $\mathcal{Q}$ is sufficiently accurate. Away from the low-energy region also for $\sigma=1.5 \Gamma$ a good agreement with $\tilde{A}_{0}$ is obtained, i.e. a sharpened structure along the cross-shaped directions with an approximately correct amplitude (as compared to figure D.2a). The strong sensitivity of especially the low-energy range on the default model may be interpreted as a result of the subtle interplay of positive and negative regions of high-amplitude kernel matrix elements for different $\left(\mathrm{i} \varphi_{m}, \mathrm{i} \omega_{n}\right)$. The structure of the matrix elements was discussed above and plotted in figure D.1. As shown in figure D.4 a problem often encountered for not well-chosen default models is apparently an increase of spectral weight in the low-energy region $|\underline{x}| \approx 0$, which exceeds the color scale used in the plots by up to a factor of three, even for moderate deviations of $\sigma_{\text {def }}$ from $\Gamma$. This is unfortunate, because not only for spectral functions unphysically high values may be deduced, but also the overall weight of the spectral function is too large. However, since the kernel $\mathcal{Q}$ imposes an exact relation on $G_{0}$ and is resolved well enough, this unfortunate aspect is identified as a pure MaxEnt ("second step") artifact. As such it is no conceptual problem of the $\mathcal{Q}$-approach and can in principle be removed by developing a more sophisticated MaxEnt algorithm which imposes the physical constraints as prior information.

From our data we can conclude that default models with the shape (D.3) are apparently not of much use for functions whose high-energy behaviour along the singular directions is a Lorentzian with width $\Gamma$. Once the high-energy structure is known to be such, an interesting experiment is to flatten out the low- to intermediateenergy structure of the default model, by imposing an $\underline{x}$-dependent

$$
\sigma_{\text {def }}\left(x_{\varphi}\right)=\Gamma+\left(\tilde{\sigma}_{\text {def }}-\Gamma\right) \frac{R^{2}}{x_{\varphi}^{2}+R^{2}},
$$

where $R$ is the flattening radius and $\tilde{\sigma}_{\text {def }}$ is a maximum flattening. For $G_{0}$ it turns out that the resulting MaxEnt solution is practically identical to the $\sigma_{\text {def }}=\Gamma$ solution. The "second step"-artifact for $G_{0}$ of overshooting values (Fig. D.4) can, in fact, be cured by using the correct high-energy limit as imposed by the $\sigma_{\operatorname{def}}\left(x_{\varphi}\right)$ function. The low-energy artifact is thus caused by missing a-priori information about the high-energy structure. This appears to be another manifestation of the fact that the kernel $\mathcal{Q}$ mixes energy scales over a comparably wide range. 


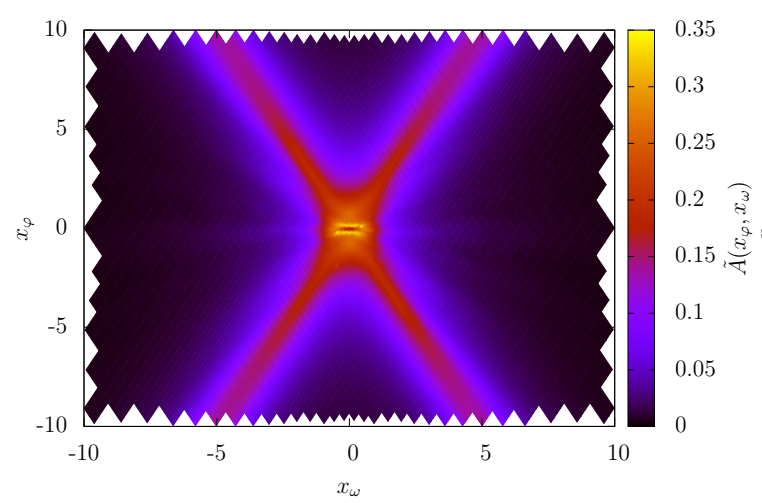

(a) $\tilde{\sigma}_{\text {def }}=1.5 \Gamma$

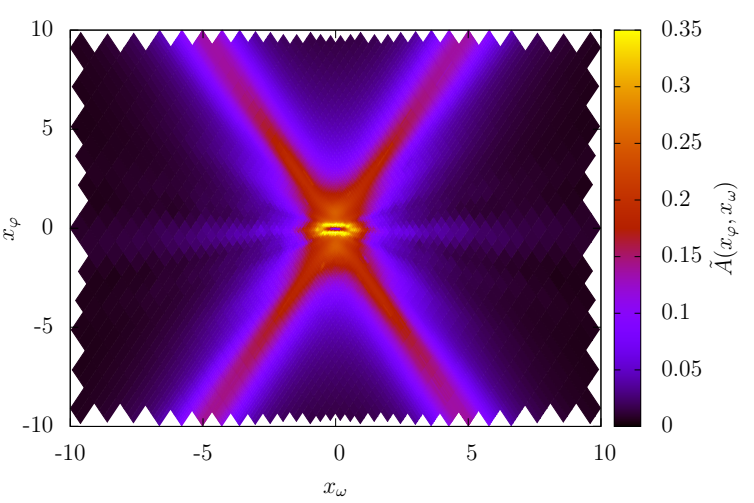

(b) $\tilde{\sigma}_{\text {def }}=3.0 \Gamma$

Figure D.4: MaxEnt results for flat low-energy default models (D.4), $R=5$. As compared to Fig. D.3b, the quality of low-energy data is increased significantly, due to the correct high-energy behaviour of the default model.

\section{D.1. Application to the interacting model}

Switching on a finite Coulomb interaction, one has to be fully aware of the fact that the $\mathcal{Q}$-mapping can no longer be expected to be exact. ${ }^{1}$ However, a special case of the assumption, namely the fitting ansatz in Ref. [27], is found to yield reasonable results which agree with other methods up to a certain extent [53]. Therefore, it seems worthwhile to investigate how far one can go with the controlled MaxEnt approach to the inversion of the $\mathcal{Q}$-mapping. ${ }^{2}$

\section{Lateral structure along singular directions}

As shown in the preceding section, the determination of an approximate a-priori picture of the high-energy structure is crucial for the success of the MaxEnt procedure. As sketched in figure D.5, at finite $U$, one may, for example (panel a of the figure), expect the lateral structure be an unphysical copy of a spectral function, i.e. two Hubbard peaks with possibly an additional peak associated to a quasi-particle resonance. Such a structure would extend over a range $\approx U$. However, the two parallel Hubbard peaks can be expected to approximately have a Lorentzian structure of width $\approx \Gamma$ and would generate a type of branch cut in the $\mathcal{Q}$-mapping which is equivalent to the

\footnotetext{
${ }^{1}$ See the detailed discussion in sections 7.2 .2 and appendix B.

${ }^{2}$ To be more precise, for technical reasons (conservation of the spectral function normalization for any causal selfenergy) the fits in Refs. [27; 53] were performed with respect to the selfenergy, not with respect to the Green's function. Because the $\mathcal{Q}$-mapping is also exact for ansatz functions of the fits presented in [27], the fits are essentially special cases of the present work, though. The first-order Padé approximant fits presented in Ref. [53] appear to represent a different kind of generalization.
} 


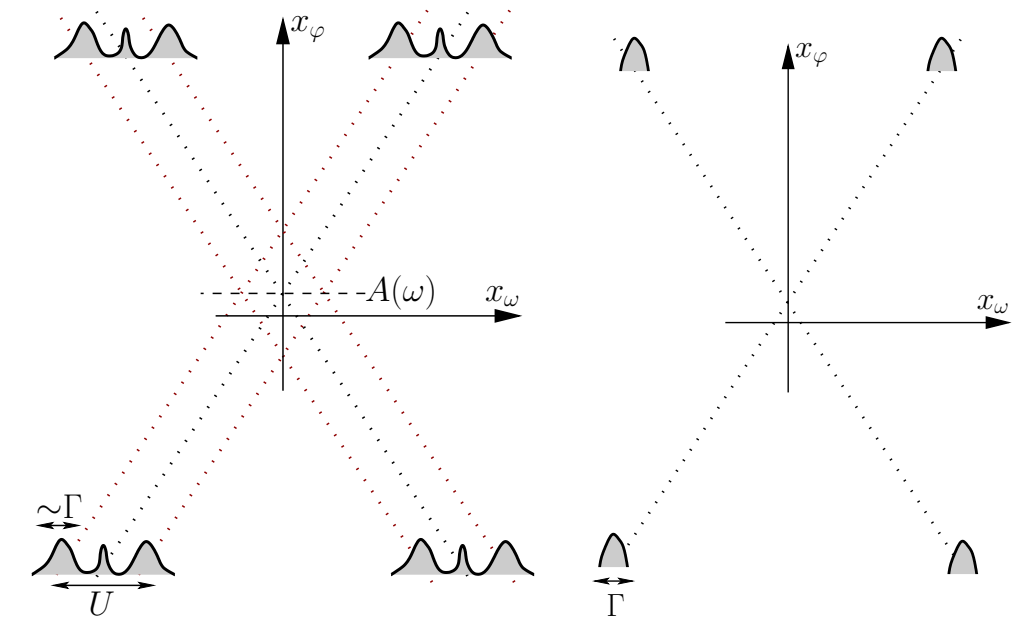

(a) nontrivial physics at $x_{\omega} \approx \pm x_{\varphi} / 2 \quad$ (b) "asymptotic freedom"

Figure D.5: At high energies, one might (a) expect the lateral structure of $\tilde{A}\left(x_{\varphi}, x_{\omega}\right)$ to be composed of two Hubbard peaks and possibly a quasi-particle resonance which combine to the physical spectrum $A(\omega)$ at the intersection point. In the complementary scenario (b), the function $\tilde{A}$ would not differ from the noninteracting one at high energies.

one in $G_{0}$. This would effectively yield the picture provided by panel (b) of figure D.5. One may also have to consider that in the strongly correlated regime, Hubbard satellites may be broadened up to a width of $2 \Gamma$, due to many-body correlations [128].

It is a priori uncertain to which extent either of the intuitive pictures in figure D.5 is correct. However, maybe the foremost conceptual strength of the $\mathcal{Q}$-mapping is the precise rendering of the high-energy structure of the imaginary-voltage theory (appendix B). One can expect that only a characteristic width of the lateral structure along the singular directions is needed in order to model the correct high-energy contribution to the amplitude of the discontinuity of $G\left(z_{\varphi}, z_{\omega}\right)$ at the low-to-intermediate energy portions of the branch cuts. Based on this, we can investigate the posterior probability $\operatorname{Pr}\left(\sigma_{\text {def }} \mid \bar{G}\right)$ for default models (D.3) as a function of their width $\sigma_{\text {def }}$.

The thus determined most probable $\sigma_{\text {def }}$ could then serve as evidence about an effective description of the high-energy structure $\tilde{A}\left(x_{\varphi}, x_{\omega}\right)$ for the actual computations. However, only low- to intermediate-energy data are available as input data from the QMC simulations. Therefore, the posterior probability probe with respect to default models (D.3) has to be interpreted with care. In figure D.6 posterior probabilities for different interaction strengths are displayed. Due to the width being significantly larger than $2 \Gamma$ for $U=15 \Gamma$ it is obvious that the lateral width cannot solely be interpreted as a signature of the Hubbard bands. Merely, the overall Lorentzian broadness of the spectral function seems to be obtained. Based on our data, neither of the scenarios D.5 can be preferred. However, based on our experi- 


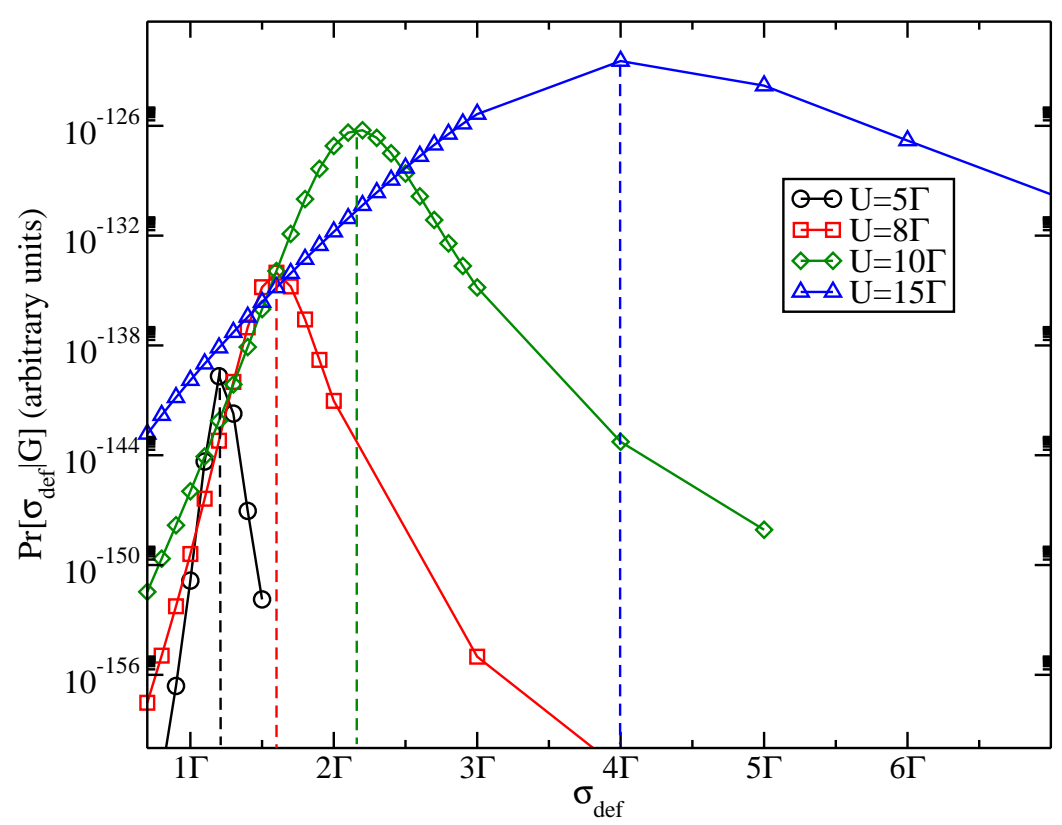

Figure D.6: Posterior probability of the default model (D.3) at $\beta \Gamma=5, e \Phi=\Gamma$ for several interactions strengths. The result is found to be essentially independent of the bias voltage. The kernel validated in figure D.2d has been used.

ence, the most probable high-energy structure also yields reasonable results in the case of comparably strong interactions. 
D. MaxEnt implementation for data from multiple wedges 


\section{E. Numerical representation of the multi-wedge map $\mathcal{Q}$}

In this appendix, a recipe for the numerical computation of the quadruple integral $\mathcal{Q}$ is given. The application to the test function (7.17), $f_{\underline{X}, \delta}$, for a function value at $\left(\mathrm{i} \varphi_{m}, \mathrm{i} \omega_{n}\right)$ is to be computed. The first three integrals can be computed analytically by use of a computer algebra system. A numerical quadrature method can be used for the approximation of the remaining integral.

\section{E.1. Analytic computation of three first integrals}

Using the translational and scale invariance of the edge-to-edge contribution $\mathcal{Q}_{\vartheta}^{\text {(edge) }}$, only the action of $\mathcal{Q}_{\vartheta}^{(\text {edge) }}$ on our test function

$$
f(x, y)=\frac{1}{\pi^{2}} \frac{1}{(y-x / 2)^{2}+1} \cdot \frac{1}{(y+x / 2)^{2}+1}
$$

has to be computed, yielding the results shown in figure 7.4. For brevity we set $y=x_{\omega}$ and $x=x_{\varphi}$. The first principal integral can be eliminated by straightforward application of the residue theorem:

$$
\begin{aligned}
(\mathcal{H} f)(x, \tilde{y}) & =\not d \mathrm{~d} y \frac{\pi^{-1}}{\tilde{y}-y} f(x, y) \\
& =-\frac{8}{\pi^{2}} \frac{\left(x^{2}-12-4 \tilde{y}^{2}\right) \tilde{y}}{\left(16 \tilde{y}^{4}+32 \tilde{y}^{2}-8 \tilde{y}^{2} x^{2}+16+8 x^{2}+x^{4}\right)\left(x^{2}+4\right)} .
\end{aligned}
$$

As a next step, introducing the short-hand notation $s=\sin \vartheta$ and $c=\cos \vartheta$, imposing the rotation operator $\mathcal{R}_{\vartheta}^{-1}$, one obtains

$$
\left(\mathcal{R}_{\vartheta}^{-1} \mathcal{H} f\right)(k, l)=\operatorname{subs}(x \rightarrow k c+l s, \tilde{y} \rightarrow l c-k s ;(\mathcal{H} f)(x, \tilde{y})) .
$$

Here, "subs" denotes the operation of a variable substitution. In order to apply the second Hilbert transform, it is necessary to determine the poles of the corresponding 
integrand

$$
\begin{aligned}
g(k, \tilde{\tilde{y}} ; l):= & \frac{\pi^{-1}}{\tilde{\tilde{y}}-l} \cdot\left(\mathcal{R}_{\vartheta}^{-1} \mathcal{H} f\right)(k, l) \\
= & 8 \frac{\left(k^{2} c^{2}+10 k c l s+l^{2} s^{2}-12-4 l^{2} c^{2}-4 k^{2} s^{2}\right)(l c-k s)}{\pi^{3}\left(k^{2} c^{2}+2 k c l s+l^{2} s^{2}+4\right)} \\
& \cdot\left(4 l^{2} c^{2}-6 k c l s+4 k^{2} s^{2}+4+4 k c^{2} l-\right. \\
& \left.\quad-4 k^{2} c s+4 l^{2} s c-4 l s^{2} k+k^{2} c^{2}+l^{2} s^{2}\right)^{-1} . \\
& \cdot(-\tilde{\tilde{y}}+l)^{-1}
\end{aligned}
$$

with respect to the integration variable $l$. One finds that the function has the following seven poles in the complex plane:

$$
\begin{aligned}
l_{1} & :=\tilde{\tilde{y}} \\
l_{2,3} & :=\frac{-k c+2 k s \pm 2 \mathrm{i}}{s} \\
l_{4,5} & :=\frac{-k c+2 k s \pm 2 \mathrm{i}}{s+2 c} \\
l_{6,7} & :=\frac{k c+2 k s \pm 2 \mathrm{i}}{-s+2 c} .
\end{aligned}
$$

The Hilbert transform can now be evaluated through the residue sum

$$
\left(\mathcal{H R}_{\vartheta}^{-1} \mathcal{H} f\right)(k, \tilde{\tilde{y}})=\sum_{n=1}^{7} \operatorname{Re}\left[2 \pi \mathrm{i} \cdot \operatorname{Res}_{l=l_{n}} g(k, \tilde{\tilde{y}} ; l)\right] \cdot \theta\left(\operatorname{Im} l_{n}\right)
$$

The Heaviside function $\theta(x)$ ensures that only poles from the upper half plane are taken into account for the evaluation of the contour integral which corresponds to the principal value integral. An explicit evaluation of the residue sum (E.9) can be accomplished with a computer algebra system. A dramatic increase in complexity is coming along with the constraints $\operatorname{Im} l_{n}>0$ which depend on the wedge orientation angle $\vartheta$. In fact, six separate cases emerge as a function of $\vartheta$. They can be parametrized by three overlapping different cases, namely (A) terms which are proportional to $\operatorname{sgn}(s)$, (B) proportional to $\operatorname{sgn}(2 c+s)$, and (C) terms which are proportional to $\operatorname{sgn}(2 c-s)$, discriminating between the different signs of $\operatorname{Im} l_{n}$. The term

$$
\left(\mathcal{R}_{\vartheta} \mathcal{H} \mathcal{R}_{\vartheta}^{-1} \mathcal{H} f\right)(x, y)=\operatorname{subs}\left(k \rightarrow x c-y s, \tilde{\tilde{y}} \rightarrow y c+x s ;\left(\mathcal{H} \mathcal{R}_{\vartheta}^{-1} \mathcal{H} f\right)(k, \tilde{\tilde{y}})\right)
$$


is then best reorganized into rational functions as coefficients of the sign functions,

$$
\begin{aligned}
\left(\mathcal{Q}_{\vartheta}^{(\text {edge })} f\right)(x, y)=\left(\mathcal{R}_{\vartheta} \mathcal{H} \mathcal{R}_{\vartheta}^{-1} \mathcal{H} f\right)(x, y)= & A(x, y) \cdot \operatorname{sgn}(s)+ \\
& +B(x, y) \cdot \operatorname{sgn}(2 c+s)+ \\
& +C(x, y) \cdot \operatorname{sgn}(2 c-s) .
\end{aligned}
$$

This explicit split is necessary for the study of the interplay of the rational functions $A(x, y), B(x, y)$, and $C(x, y)$ in a computer algebra system.

\section{E.1.1. Rational coefficients of the transformed edge test function}

The rational functions

$$
\begin{aligned}
& A(x, y)=\frac{A_{\text {enum }}(x, y)}{A_{\text {denom }}(x, y)}, \\
& B(x, y)=\frac{B_{\text {enum }}(x, y)}{B_{\text {denom }}(x, y)}, \\
& C(x, y)=\frac{C_{\text {enum }}(x, y)}{C_{\text {denom }}(x, y)} .
\end{aligned}
$$

have the following polynomials as enumerators and denominators:

$$
\begin{aligned}
A_{\text {enum }}(x, y)=- & \left(\left(-1+x^{2}+1 / 4 y^{2}-\frac{41}{16} x y\right) c^{4}-\frac{13}{16}\left(\frac{12}{13} x y+x^{2}+\frac{12}{13}-\frac{28}{13} y^{2}\right) s c^{3}\right. \\
+ & \left(1+\frac{13}{8} x y-1 / 2 y^{2}\right) c^{2}+1 / 16 s\left(-4+x^{2}-4 x y-12 y^{2}\right) c \\
- & 1 / 16 y(-4 y+x)) \\
& A_{\text {denom }}(x, y)=\pi^{2}\left(x^{2}+4-4 x y+4 y^{2}\right) \cdot K(x, y), \\
B_{\text {enum }}(x, y)=- & \left(\left(-1+x^{2}+1 / 4 y^{2}+\frac{41}{16} x y\right) c^{4}+\right. \\
& +\frac{13}{16} s\left(-\frac{12}{13} x y+x^{2}+\frac{12}{13}-\frac{28}{13} y^{2}\right) c^{3}+\left(1-\frac{13}{8} x y-1 / 2 y^{2}\right) c^{2} \\
& \left.-1 / 16 s\left(-4+x^{2}+4 x y-12 y^{2}\right) c+1 / 16 y(4 y+x)\right)
\end{aligned}
$$




$$
\begin{aligned}
& B_{\text {denom }}(x, y)=\left(x^{2}+4 x y+4 y^{2}+4\right) \pi^{2} \cdot K(x, y), \\
C_{\text {enum }}(x, y)= & -3\left(-1 / 3 y^{2}-3+x^{2}\right) y x c^{4}-s\left(\left(-3 x^{2}+4\right) y^{2}-5 x^{2}+4+x^{4}\right) c^{3} \\
+ & 3\left(-10 / 3+x^{2}-2 / 3 y^{2}\right) y x c^{2} \\
+ & \left(\left(-3 x^{2}+4\right) y^{2}-x^{2}+4\right) s c+y x\left(y^{2}+1\right),
\end{aligned}
$$

and

$$
C_{\text {denom }}(x, y)=\left(x^{2}+4\right) \pi^{2} \cdot K(x, y) .
$$

For the denominators, we introduced the shared polynomial

$$
\begin{aligned}
K(x, y)=\left(y^{4}+\right. & \left.\left(-3-6 x^{2}\right) y^{2}+3 x^{2}-4+x^{4}\right) c^{4} \\
& -4\left(3 / 2+x^{2}-y^{2}\right) x s y c^{3}+\left(-2 y^{4}+\left(2+6 x^{2}\right) y^{2}+4+x^{2}\right) c^{2} \\
& -4\left(1 / 2+y^{2}\right) x s y c+y^{2}+y^{4} .
\end{aligned}
$$

\section{E.1.2. Composition of the rational coefficients}

In contrast to the actual edge functions $\mathcal{Q}_{\vartheta}^{(\text {edge })} f$, the coefficients $A(x, y), B(x, y)$, and $C(x, y)$ are comparably ill-behaved. For example, one obtains

$$
\left.B(x, y)\right|_{\vartheta=0}=-8 \frac{x+y}{\pi^{2}\left(x^{2}+4\right)\left(x^{2}+4 x y+4 y^{2}+4\right)} \cdot \frac{1}{x} .
$$

Apparently, the function diverges for $x \rightarrow 0$. The functions $A(x, y)$ and $C(x, y)$ are similarly structured. However, the actual edge functions, as displayed in figure 7.4, have no such singularities, but do rather represent smooth deformations of the test function when the wedge orientation angle $\vartheta$ is tuned. As a consequence, the real singularities are cancelled as the rational functions are added up in equation (E.11). For a consistent further evaluation of the action of $\mathcal{Q}$ on the test function it is thus necessary to study each of the full combinations (E.11) separately. There are six possible combinations, namely the sectors

$$
\begin{aligned}
& \text { (a) } \quad 0 \quad \leq \vartheta \leq \arctan 2 \text {, } \\
& \text { (b) } \arctan 2 \leq \vartheta \leq \pi-\arctan 2 \text {, } \\
& \text { (c) } \pi-\arctan 2 \leq \vartheta \leq \pi \text {, } \\
& \text { (d) } \quad \pi \quad \leq \vartheta \leq \pi+\arctan 2 \text {, } \\
& \text { (e) } \pi+\arctan 2 \leq \vartheta \leq 2 \pi-\arctan 2 \text {, } \\
& \text { (f) } 2 \pi-\arctan 2 \leq \vartheta \leq 2 \pi \text {. }
\end{aligned}
$$


For example, the expression for the sector $(a)$ reads

$$
\left.\left(\mathcal{Q}_{\vartheta}^{(\text {edge })} f\right)\right|_{(a)}(x, y)=A(x, y)+B(x, y)+C(x, y) .
$$

\section{E.1.3. Contraction with the Poisson kernel}

As a next step, one of the integrals introduced by Vladimirov's Poisson kernel (5.49) will be evaluated analytically. For this, the pole structure of both, the Poisson kernel (5.50), and the edge-transformed test function have to be analyzed.

\section{Pole structure of edge-transformed test functions}

In fact, we are interested in the pole structure of the scaled and then translated edge-transformed test functions $\left(\mathcal{T}_{\underline{X}} \Lambda_{1 / \delta} \mathcal{Q}_{\vartheta}^{\text {(edge) }} f\right)\left(x^{\prime}, y^{\prime}\right)$, from equation (7.20). The poles and also the residues of these functions are however easily calculated from the poles and residues of $\left(\mathcal{Q}_{\vartheta}^{(\text {edge) }} f\right)(x, y)$. This is the crucial advantage of translational and scale invariance of $\mathcal{Q}_{\vartheta}^{\text {(edge) }}$.

For example, in sector $(a)$, the following poles of $\left(\mathcal{Q}_{\vartheta}^{(\text {edge })} f\right)(x, y)$ with respect to $x$ are obtained: $x_{1,2}= \pm 2 \mathrm{i}, x_{3,4}=s y \pm 2 \mathrm{i}, x_{5,6}=-2 y \pm 2 \mathrm{i}, x_{7,8}=2 y \pm 2 \mathrm{i}$. The corresponding residues are $r_{1,2}=\frac{1}{2 \pi^{2} y}, r_{3,4}=-\frac{1}{2 \pi^{2} y}, r_{5,6}=\frac{1}{4 \pi^{2}} \cdot \frac{-1 \mp \mathrm{i} y}{\left(y^{2}+1\right) y}, r_{7,8}=$ $\frac{1}{4 \pi^{2}} \cdot \frac{1 \mp \mathrm{i} y}{\left(y^{2}+1\right) y}$. The resulting residues of $\left(\mathcal{T}_{\underline{X}} \Lambda_{1 / \delta} \mathcal{Q}_{\vartheta}^{(\text {edge) }} f\right)\left(x^{\prime}, y^{\prime}\right)$ are then given by

$$
r_{i}^{\prime}=\frac{1}{\delta} \cdot \operatorname{subs}\left(y \rightarrow \frac{y^{\prime}-Y}{\delta} ; r_{i}(y)\right)
$$

where the center of mass of the test function is $\underline{X}=(X, Y)$. They are associated to the poles of $\left(\mathcal{T}_{\underline{X}} \Lambda_{1 / \delta} \mathcal{Q}_{\vartheta}^{(\text {edge })} f\right)\left(x^{\prime}, y^{\prime}\right)$, which are similarly given by

$$
x_{i}^{\prime}=X+\delta \cdot \operatorname{subs}\left(y \rightarrow \frac{y^{\prime}-Y}{\delta} ; x_{i}\right) .
$$

In order to obtain the true residues with respect to the $x^{\prime}$-contraction with the Poisson kernel, one only has to evaluate the Poisson kernel at the poles (5.50) and multiply $r_{i}^{\prime}$ with the value.

\section{Pole structure of Poisson kernel}

The pole structure of Vladimirov's Poisson kernel is rather straightforward to compute, however rather lengthy expressions result for the poles. Similar to above, the residues have to be multiplied by the function values of the edge-transformed test function $\left(\mathcal{T}_{\underline{X}} \Lambda_{1 / \delta} \mathcal{Q}_{\vartheta}^{\text {(edge) }} f\right)\left(x^{\prime}, y^{\prime}\right)$ at the pole of the Poisson kernel. Poles of the rotated 
Poisson kernel $\mathcal{P}_{r, \vartheta}\left(\varphi_{m}, \omega_{n} ; x^{\prime}, y^{\prime}\right)$ with respect to $x^{\prime}$ are

$$
\begin{aligned}
x_{1,2}^{\prime} & =\frac{s-c \varepsilon}{s \varepsilon+c} y^{\prime} \pm \mathrm{i} \frac{\eta_{1}+\varepsilon \eta_{2}}{s \varepsilon+c}, \\
x_{3,4}^{\prime} & =\frac{c \varepsilon+s}{c-s \varepsilon} y^{\prime} \pm \mathrm{i} \frac{\eta_{1}-\varepsilon \eta_{2}}{s \varepsilon-c} .
\end{aligned}
$$

We introduced the short-hand notations $\eta_{1}=\varphi_{m} c-\omega_{n} s, \eta_{2}=\varphi_{m} s+\omega_{n} c$. The associated residues of $\mathcal{P}_{r, \vartheta}$ are easily determined.

\section{Residue sum for the $x^{\prime}$ integral}

An algebraic expression for the residue sum corresponding to the $x^{\prime}$ integral can be generated symbolically by evaluating

$$
\begin{aligned}
& I_{3}\left(\varphi_{m}, \omega_{n} ; y^{\prime}\right)= \int \mathrm{d} x^{\prime} \underbrace{\mathcal{P}_{r, \vartheta}\left(\varphi_{m}, \omega_{n} ; x^{\prime}, y^{\prime}\right)}_{\text {"Poisson" }} \cdot \underbrace{\left(\mathcal{T}_{\underline{X}} \Lambda_{1 / \delta} \mathcal{Q}_{\vartheta}^{(\text {edge })} f\right)\left(x^{\prime}, y^{\prime}\right)}_{\text {"edge" }}= \\
&= \sum_{x_{i}^{\prime}}^{\text {"edge" }} \operatorname{Re}\left[2 \pi \mathrm{i} \cdot \mathcal{P}_{r, \vartheta}\left(\varphi_{m}, \omega_{n} ; x_{i}^{\prime}, y^{\prime}\right) \cdot r_{i}^{\prime}\right] \cdot \theta\left(\operatorname{Im} x_{i}^{\prime}\right)+ \\
&+\sum_{x_{i}^{\prime}}^{\text {"Poisson" }} \operatorname{Re}\left[2 \pi \mathrm{i} \cdot\left(\mathcal{T}_{\underline{X}} \Lambda_{1 / \delta} \mathcal{Q}_{\vartheta}^{(\text {edge })} f\right)\left(x_{i}^{\prime}, y^{\prime}\right) \cdot\right. \\
&\left.\cdot \operatorname{Res}_{x^{\prime}=x_{i}^{\prime}} \mathcal{P}_{r, \vartheta}\left(\varphi_{m}, \omega_{n} ; x^{\prime}, y^{\prime}\right)\right] \cdot \theta\left(\operatorname{Im} x_{i}^{\prime}\right) .
\end{aligned}
$$

However, it yields rather lengthy formulae, because the integrations with respect to $x^{\prime}$ have to be done separately, for each of the sectors (E.23) to (E.28). The growth in complexity is also due to amount of parameters which increased dramatically by introducing the Poisson kernel and inserting the translations $\mathcal{T}_{\underline{X}}$ and scaling $\Lambda_{1 / \delta}$ of the edge functions. It is possible to export the expressions resulting from (E.34) from the computer algebra system to a file of Fortran code which is 500 kilobytes in size. Similar to the procedure described in section E.1.2 for the second integral, the expression (E.34) includes removable discontinuities.

\section{E.2. Numerical quadrature of the fourth integral}

Due to the vast complexity of expression (E.34), the last remaining integral

$$
I_{4}\left(\varphi_{m}, \omega_{n}\right)=\int \mathrm{d} y^{\prime} I_{3}\left(\varphi_{m}, \omega_{n} ; y^{\prime}\right)
$$




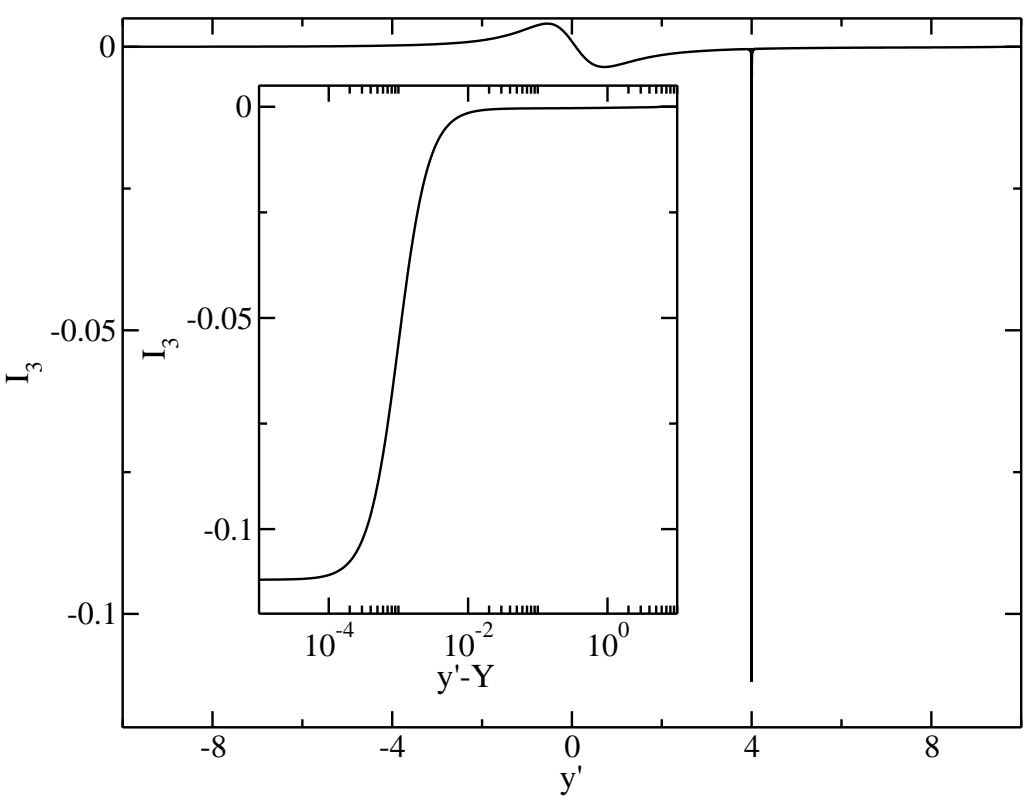

Figure E.1: Example for $I_{3}$ as a function of $y^{\prime}$, using $\varphi_{m}=2.0, \omega_{n}=2.0$, with test function location $X=0.0, Y=4.0$, and test function width $\delta=10^{-3}$. The interacting branch cut geometry is used for the determination of $r$ and $\vartheta$.

is evaluated numerically, making use of the exported Fortran code. Adaptive integration routines from the GNU Scientific Library are imposed [129]. Because the resulting matrix elements give rise to an inverse problem, it is compulsory to achieve a high integration accuracy. By definition, the integrand is most distinguishly structured in the area $y^{\prime} \approx Y$, on a scale $\delta$. Special attention has to be drawn to the appropriate integration of this range.

The high-frequency tails $(-\infty,-R]$ and $[R, \infty)$ need to be integrated out separately, where $R$ is the integration range of the conventional quadrature. For some parameter values of $\delta, \underline{X}$, etc., problems with the convergence of these high-energy integrals may occur, due to floating point precision. Choosing a finite interval extending to $\pm \max \left(10^{6},|X| \cdot 10^{3},|Y| \cdot 10^{3}\right)$ is then usually sufficient for numerically satisfactory data.

A typical shape of the integrand $I_{3}$ is shown in figure E.1. The structure at $y^{\prime} \approx Y$ is not necessarily $\delta$-shaped, but depending on the values of $\varphi_{m}$ and $\omega_{n}$ it may rather look like the Hilbert transform of such. The integral may be computed at each point $(X, Y)$ of the $\tilde{A}$ discretization lattice for all values of the simulation data $\left(\mathrm{i} \varphi_{m}, \mathrm{i} \omega_{n}\right)$ on a computer cluster. In practice, the computation of the matrix elements has to be done only once for each temperature $\beta$, regardless of the bias voltage. This is because an adjustment of the $\tilde{A}$ grid is not necessary in the latter case.

In future applications, one should aim at symbolically programming the residue sum of the fourth integral and then take the limit $\delta \rightarrow 0$ analytically. 
E. Numerical representation of the multi-wedge map $\mathcal{Q}$ 


\section{Bibliography}

[1] W.J. De Haas and G.J. van dën Berg, "The electrical resistance of gold and silver at low temperatures," Physica, vol. 3, no. 6, pp. 440 - 449, 1936.

[2] W.J. de Haas, J. de Boer, and G.J. van dën Berg, "The electrical resistance of

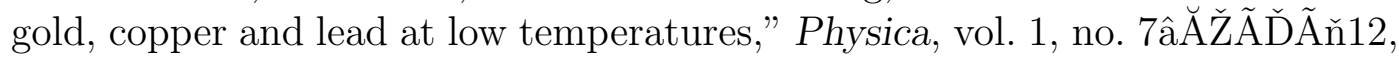
pp. 1115 - 1124, 1934.

[3] J. Kondo, "Resistance Minimum in Dilute Magnetic Alloys," Progress of Theoretical Physics, vol. 32, pp. 37-49, 1964.

[4] Ralf Bulla, Theo A. Costi, and Thomas Pruschke, "Numerical renormalization group method for quantum impurity systems," Rev. Mod. Phys., vol. 80, no. 2, pp. 395-450, Apr 2008.

[5] Kenneth G. Wilson, "The renormalization group: Critical phenomena and the Kondo problem," Rev. Mod. Phys., vol. 47, pp. 773-840, Oct 1975.

[6] A.C. Hewson, The Kondo Problem to Heavy Fermions, Cambridge University Press, New York, N.Y., 1993.

[7] D. Goldhaber-Gordon, Hadas Shtrikman, D. Mahalu, David Abusch-Magder, U. Meirav, and M. A. Kastner, "Kondo effect in a single-electron transistor," vol. 391, no. 6663, pp. 156-159, Jan. 1998.

[8] Sara M. Cronenwett, Tjerk H. Oosterkamp, and Leo P. Kouwenhoven, "A Tunable Kondo Effect in Quantum Dots," Science, vol. 281, no. 5376, pp. 540-544, 1998.

[9] Daniel Loss and David P. DiVincenzo, "Quantum computation with quantum dots," Phys. Rev. A, vol. 57, no. 1, pp. 120-126, Jan 1998.

[10] R. Hanson, L. P. Kouwenhoven, J. R. Petta, S. Tarucha, and L. M. K. Vandersypen, "Spins in few-electron quantum dots," Rev. Mod. Phys., vol. 79, pp. 1217-1265, Oct 2007.

[11] Selman Hershfield, John H. Davies, and John W. Wilkins, "Probing the Kondo resonance by resonant tunneling through an Anderson impurity," Phys. Rev. Lett., vol. 67, pp. 3720-3723, Dec 1991. 
[12] Selman Hershfield, John H. Davies, and John W. Wilkins, "Resonant tunneling through an Anderson impurity. I. Current in the symmetric model," Phys. Rev. B, vol. 46, pp. 7046-7060, Sep 1992.

[13] Tatsuya Fujii and Kazuo Ueda, "Perturbative approach to the nonequilibrium Kondo effect in a quantum dot," Phys. Rev. B, vol. 68, pp. 155310, Oct 2003.

[14] Herbert Schoeller and Frank Reininghaus, "Real-time renormalization group in frequency space: A two-loop analysis of the nonequilibrium anisotropic Kondo model at finite magnetic field," Phys. Rev. B, vol. 80, pp. 045117, Jul 2009.

[15] R. Gezzi, Th. Pruschke, and V. Meden, "Functional renormalization group for nonequilibrium quantum many-body problems," Phys. Rev. B, vol. 75, pp. 045324, Jan 2007.

[16] Severin G. Jakobs, Volker Meden, and Herbert Schoeller, "Nonequilibrium Functional Renormalization Group for Interacting Quantum Systems," Phys. Rev. Lett., vol. 99, pp. 150603, Oct 2007.

[17] A. Rosch, J. Paaske, J. Kroha, and P. Wölfle, "Nonequilibrium Transport through a Kondo Dot in a Magnetic Field: Perturbation Theory and Poor Man's Scaling," Phys. Rev. Lett., vol. 90, pp. 076804, Feb 2003.

[18] Dmitry Lobaskin and Stefan Kehrein, "Crossover from nonequilibrium to equilibrium behavior in the time-dependent Kondo model," Phys. Rev. B, vol. 71, pp. 193303, May 2005.

[19] Shunsuke Kirino, Tatsuya Fujii, Jize Zhao, and Kazuo Ueda, "Time-Dependent DMRG Study on Quantum Dot under a Finite Bias Voltage," Journal of the Physical Society of Japan, vol. 77, no. 8, pp. 084704, 2008.

[20] F. Heidrich-Meisner, A. E. Feiguin, and E. Dagotto, "Real-time simulations of nonequilibrium transport in the single-impurity Anderson model," Phys. Rev. B, vol. 79, pp. 235336, Jun 2009.

[21] Philipp Werner, Takashi Oka, Martin Eckstein, and Andrew J. Millis, "Weakcoupling quantum Monte Carlo calculations on the Keldysh contour: Theory and application to the current-voltage characteristics of the Anderson model," Phys. Rev. B, vol. 81, pp. 035108, Jan 2010.

[22] Frithjof B. Anders, "Steady-State Currents through Nanodevices: A ScatteringStates Numerical Renormalization-Group Approach to Open Quantum Systems," Phys. Rev. Lett., vol. 101, pp. 066804, Aug 2008. 
[23] Dvira Segal, Andrew J. Millis, and David R. Reichman, "Numerically exact path-integral simulation of nonequilibrium quantum transport and dissipation," Phys. Rev. B, vol. 82, pp. 205323, Nov 2010.

[24] S. Weiss, J. Eckel, M. Thorwart, and R. Egger, "Iterative real-time path integral approach to nonequilibrium quantum transport," Phys. Rev. B, vol. 77, pp. 195316, May 2008.

[25] Dvira Segal, Andrew J. Millis, and David R. Reichman, "Nonequilibrium transport in quantum impurity models: exact path integral simulations," Phys. Chem. Chem. Phys., vol. 13, pp. -, 2011.

[26] Marco Schiró and Michele Fabrizio, "Real-time diagrammatic Monte Carlo for nonequilibrium quantum transport," Phys. Rev. B, vol. 79, pp. 153302, Apr 2009 .

[27] J. E. Han and R. J. Heary, "Imaginary-Time Formulation of Steady-State Nonequilibrium: Application to Strongly Correlated Transport," Phys. Rev. Lett., vol. 99, no. 23, pp. 236808, Dec 2007.

[28] Dimitriu Nikolaevich Zubarev, Nonequilibrium Statistical Thermodynamics, Studies in Soviet Science. Consultants Bureau, New York, 1974.

[29] Selman Hershfield, "Reformulation of steady state nonequilibrium quantum statistical mechanics," Phys. Rev. Lett., vol. 70, no. 14, pp. 2134-2137, Apr 1993.

[30] A. N. Rubtsov, V. V. Savkin, and A. I. Lichtenstein, "Continuous-time quantum Monte Carlo method for fermions," Phys. Rev. B, vol. 72, no. 3, pp. 035122, Jul 2005.

[31] Emanuel Gull, Philipp Werner, Andrew Millis, and Matthias Troyer, "Performance analysis of continuous-time solvers for quantum impurity models," Phys. Rev. B, vol. 76, pp. 235123, Dec 2007.

[32] Philipp Werner, Takashi Oka, Martin Eckstein, and Andrew J. Millis, "Weakcoupling quantum Monte Carlo calculations on the Keldysh contour: Theory and application to the current-voltage characteristics of the Anderson model," Phys. Rev. B, vol. 81, pp. 035108, Jan 2010.

[33] Emanuel Gull, Andrew J. Millis, Alexander I. Lichtenstein, Alexey N. Rubtsov, Matthias Troyer, and Philipp Werner, "Continuous-time Monte Carlo methods for quantum impurity models," Rev. Mod. Phys., vol. 83, no. 2, pp. 349-404, May 2011. 
[34] A. G. Vitushkin (eds.) G. M. Khenkin, Several complex Variables II: Function theory in classical domains: complex potential theory, Encyclopedia of Mathematical Sciences. Springer, 1994.

[35] Mark Jarrell and J.E. Gubernatis, "Bayesian Inference and the Analytic Continuation of Imaginary-Time Quantum Monte Carlo Data," Physics Reports, vol. 269, pp. 133, May 1996.

[36] Jesper Nygard, David Henry Cobden, and Poul Erik Lindelof, "Kondo physics in carbon nanotubes," Nature, vol. 408, no. 6810, pp. 7, 2000.

[37] Pablo Jarillo-Herrero, Jing Kong, Herre S.J. van der Zant, Cees Dekker, Leo P. Kouwenhoven, and Silvano De Franceschi, "Orbital Kondo effect in carbon nanotubes," Nature, vol. 434, no. 7032, pp. 484-488, Mar. 2005.

[38] A. D. Yoffe, "Semiconductor quantum dots and related systems: Electronic, optical, luminescence and related properties of low dimensional systems," Advances in Physics, vol. 50, no. 1, pp. 1-208, 2001.

[39] S. Tomonaga, "Remarks on Bloch's Method of Sound Waves applied to ManyFermion Problems," Progress of Theoretical Physics, vol. 5, pp. 544-569, July 1950.

[40] J. M. Luttinger, "An Exactly Soluble Model of a Many-Fermion System," Journal of Mathematical Physics, vol. 4, no. 9, pp. 1154-1162, 1963.

[41] Michael Pustilnik and Leonid Glazman, "Kondo effect in quantum dots," Journal of Physics: Condensed Matter, vol. 16, no. 16, pp. R513, 2004.

[42] P. W. Anderson, "Localized Magnetic States in Metals," Phys. Rev., vol. 124, no. 1, pp. 41-53, Oct 1961.

[43] J. R. Schrieffer and P. A. Wolff, "Relation between the Anderson and Kondo Hamiltonians," Phys. Rev., vol. 149, no. 2, pp. 491-492, Sep 1966.

[44] P. W. Anderson, "Infrared Catastrophe in Fermi Gases with Local Scattering Potentials," Phys. Rev. Lett., vol. 18, no. 24, pp. 1049-1051, Jun 1967.

[45] Philip W. Anderson, "Ground State of a Magnetic Impurity in a Metal," Phys. Rev., vol. 164, no. 2, pp. 352-359, Dec 1967.

[46] H. Suhl, "Dispersion Theory of the Kondo Effect," Phys. Rev., vol. 138, no. 2A, pp. A515-A523, Apr 1965.

[47] Yosuke Nagaoka, "Self-Consistent Treatment of Kondo's Effect in Dilute Alloys," Phys. Rev., vol. 138, no. 4A, pp. A1112-A1120, May 1965. 
[48] F. D. M. Haldane, "Scaling Theory of the Asymmetric Anderson Model," Phys. Rev. Lett., vol. 40, no. 6, pp. 416-419, Feb 1978.

[49] N. E. Bickers, D. L. Cox, and J. W. Wilkins, "Self-consistent large- $N$ expansion for normal-state properties of dilute magnetic alloys," Phys. Rev. B, vol. 36, no. 4, pp. 2036-2079, Aug 1987.

[50] Th. Pruschke and N. Grewe, "The Anderson model with finite Coulomb repulsion," Zeitschrift für Physik B Condensed Matter, vol. 74, pp. 439-449, 1989, 10.1007/BF01311391.

[51] N. E. Bickers, "Review of techniques in the large- $N$ expansion for dilute magnetic alloys," Rev. Mod. Phys., vol. 59, no. 4, pp. 845-939, Oct 1987.

[52] Gerald D. Mahan, Many-particle physics, Physics of Solids and Liquids. Kluwer Academic / Plenum Publishers, New York, 2000.

[53] J. E. Han, "Imaginary-time formulation of steady-state nonequilibrium in quantum dot models," Phys. Rev. B, vol. 81, no. 24, pp. 245107, Jun 2010.

[54] J.E. Han, A. Dirks, and Th. Pruschke, "Imaginary-time quantum many-body theory out of equilibrium I: Formal equivalence to Keldysh real-time theory and calculation of static properties," to be published.

[55] P. B. Wiegmann, " Exact solution of s-d exchange model at T $=0$," JETP Letters, vol. 31, no. 7, pp. 364-370, 1980.

[56] N. Andrei, "Diagonalization of the Kondo Hamiltonian," Phys. Rev. Lett., vol. 45, no. 5, pp. 379-382, Aug 1980.

[57] P.B. Wiegmann, " Towards an exact solution of the Anderson model ," Physics Letters A, vol. 80, no. 2-3, pp. 163 - 167, 1980.

[58] Norio Kawakami and Ayao Okiji, "Exact expression of the ground-state energy for the symmetric anderson model," Physics Letters A, vol. 86, no. 9, pp. 483 - 486, 1981.

[59] A. M. Tsvelick and P. B. Wiegmann, "Exact results in the theory of magnetic alloys," Advances in Physics, vol. 32, pp. 453-713, Nov. 1983.

[60] K. Yosida and K. Yamada, "Perturbation Expansion for the Anderson Hamiltonian," Prog. Theor. Phys. Suppl., vol. 46, pp. 244, 1970.

[61] K. Yosida and K. Yamada, "Perturbation Expansion for the Anderson Hamiltonian. III," Prog. Theor. Phys., vol. 53, pp. 1286-1301, 1975. 
[62] K. Yamada, "Perturbation Expansion for the Anderson Hamiltonian. II," Prog. Theor. Phys., vol. 53, pp. 970-986, 1975.

[63] B. Horvatić and V. Zlatić, "Equivalence of the perturbative and Bethe-Ansatz solution of the symmetric Anderson Hamiltonian," Journal de Physique, vol. 46, no. 9, pp. 1459-1467, 1985.

[64] K. Ueda and W. Apel, "On the ground state energy of the symmetric Anderson model," J. Phys. C: Solid State Phys., vol. 16, pp. 849, 1983.

[65] M. Salomma, "Resonance formation in the symmetric Anderson model," Solid State Communications, vol. 38, no. 9, pp. 815 - 818, 1981.

[66] B. Horvatić, D. Sokcević, and V. Zlatić, "Finite-temperature spectral density for the Anderson model," Phys. Rev. B, vol. 36, no. 1, pp. 675-683, Jul 1987.

[67] R. N. Silver, J. E. Gubernatis, D. S. Sivia, and M. Jarrell, "Spectral densities of the symmetric Anderson model," Phys. Rev. Lett., vol. 65, no. 4, pp. 496-499, Jul 1990.

[68] Henning Prüser, Martin Wenderoth, Piet E. Dargel, Alexander Weismann, Robert Peters, Thomas Pruschke, and Rainer G. Ulbrich, "Long-range Kondo signature of a single magnetic impurity," Nature Physics, vol. 7, no. 3, pp. 203-206, Mar. 2011.

[69] J Eckel, F Heidrich-Meisner, S G Jakobs, M Thorwart, M Pletyukhov, and R Egger, "Comparative study of theoretical methods for non-equilibrium quantum transport," New Journal of Physics, vol. 12, no. 4, pp. 043042, 2010.

[70] L. Kouwenhoven and L. Glazman, "Revival of the Kondo effect," Physics World, vol. 14, pp. 33-38, 2001.

[71] Antoine Georges, Gabriel Kotliar, Werner Krauth, and Marcelo J. Rozenberg, "Dynamical mean-field theory of strongly correlated fermion systems and the limit of infinite dimensions," Rev. Mod. Phys., vol. 68, no. 1, pp. 13, Jan 1996.

[72] John W. Negele and Henri Orland, Quantum Many-Particle Systems, Advanced Book Classics. Perseus Books, Reading, Massachusetts, 1998.

[73] Henrik Bruus and Karsten Flensberg, Many-Body Quantum Theory in Condensed Matter Physics: An Introduction (Oxford Graduate Texts), Oxford University Press, USA, illustrated edition edition, Nov. 2004.

[74] Jørgen Rammer, Quantum Field Theory of Non-Equilibrium States, Cambridge University Press, Cambridge, UK, 2007. 
[75] Valeriu Moldoveanu, Horia D Cornean, and Claude-Alain Pillet, "Nonequilibrium steady-states for interacting open systems: exact results," Tech. Rep. arXiv:1104.5399, May 2011.

[76] J.R. Taylor, Scattering Theory: The Quantum Theory of Nonrelativistic Collisions, R.E. Krieger Pub. Co., 1972.

[77] M. Gell-Mann and M. L. Goldberger, "The Formal Theory of Scattering," Phys. Rev., vol. 91, no. 2, pp. 398-408, Jul 1953.

[78] J. Veguillas and J.G. Muga, "On the Lippmann-Schwinger equation in Liouville space. $\dot{\Pi}$ subdynamics," Physica A: Statistical Mechanics and its Applications, vol. 150 , no. 1 , pp. $172-198,1988$.

[79] Gerard G. Emch, Algebraic Methods in Statistical Mechanics and Quantum Field Theory, Interscience Monographs and texts in physics and astronomy. Wiley Interscience, Rochester, New York, 1998.

[80] A.F Verbeure and E.J Verboven, "States of infinitely many oscillators," Physica, vol. 37, no. 1 , pp. $1-22,1967$.

[81] Benjamin Doyon, "The density matrix for quantum impurities out of equilibrium," lecture notes for the Fifth Capri Spring School on Transport in nanostructures, http://tfp1. physik. uni-freiburg. de/Capri09.

[82] Prasenjit Dutt, Jens Koch, Jong Han, and Karyn Le Hur, "Effective equilibrium theory of nonequilibrium quantum transport," Annals of Physics, vol. 326, no. 12 , pp. $2963-2999,2011$.

[83] Benjamin Doyon and Natan Andrei, "Universal aspects of nonequilibrium currents in a quantum dot," Phys. Rev. B, vol. 73, no. 24, pp. 245326, Jun 2006.

[84] J. E. Han, "Mapping of strongly correlated steady-state nonequilibrium system to an effective equilibrium," Phys. Rev. B, vol. 75, no. 12, pp. 125122, Mar 2007.

[85] P. Fendley, F. Lesage, and H. Saleur, "A unified framework for the Kondo problem and for an impurity in a Luttinger liquid," J. Statist. Phys., vol. 85, pp. 211, 1996.

[86] Ryogo Kubo, "Statistical-Mechanical Theory of Irreversible Processes. I. General Theory and Simple Applications to Magnetic and Conduction Problems," Journal of the Physical Society of Japan, vol. 12, no. 6, pp. 570-586, 1957.

[87] Paul C. Martin and Julian Schwinger, "Theory of Many-Particle Systems. I," Phys. Rev., vol. 115, no. 6, pp. 1342-1373, Sep 1959. 
[88] Yigal Meir and Ned S. Wingreen, "Landauer formula for the current through an interacting electron region," Phys. Rev. Lett., vol. 68, no. 16, pp. 2512-2515, Apr 1992.

[89] A.L. Fetter and J.D. Walecka, Quantum theory of many-particle systems, Dover Books on Physics. Dover Publications, 2003.

[90] Andreas Dirks, Philipp Werner, Mark Jarrell, and Thomas Pruschke, "Continuous-time quantum Monte Carlo and maximum entropy approach to an imaginary-time formulation of strongly correlated steady-state transport," Phys. Rev. E, vol. 82, no. 2, pp. 026701, Aug 2010.

[91] J. E. Hirsch and R. M. Fye, "Monte Carlo Method for Magnetic Impurities in Metals," Phys. Rev. Lett., vol. 56, pp. 2521, 1986.

[92] K. Mikelsons, A. Macridin, and M. Jarrell, "Relationship between Hirsch-Fye and weak-coupling diagrammatic quantum Monte Carlo methods," Phys. Rev. E, vol. 79, no. 5, pp. 057701, May 2009.

[93] E. Gull, P. Werner, O. Parcollet, and M. Troyer, "Continuous-time auxiliaryfield Monte Carlo for quantum impurity models," EPL (Europhysics Letters), vol. 82, no. 5, pp. 57003, 2008.

[94] S. M. A. Rombouts, K. Heyde, and N. Jachowicz, "Quantum Monte Carlo Method for Fermions, Free of Discretization Errors," Phys. Rev. Lett., vol. 82, pp. 4155-4159, May 1999.

[95] Matthias Troyer and Uwe-Jens Wiese, "Computational Complexity and Fundamental Limitations to Fermionic Quantum Monte Carlo Simulations," Phys. Rev. Lett., vol. 94, pp. 170201, May 2005.

[96] Philipp Werner, Armin Comanac, Luca de' Medici, Matthias Troyer, and Andrew J. Millis, "Continuous-Time Solver for Quantum Impurity Models," Phys. Rev. Lett., vol. 97, pp. 076405, Aug 2006.

[97] J.W. Negele and H. Orland, Quantum many-particle systems, Advanced Books Classics. Perseus Books, 1998.

[98] Gordon Baym and N. David Mermin, "Determination of Thermodynamic Green's Functions," Journal of Mathematical Physics, vol. 2, no. 2, pp. 232-234, 1961.

[99] V. S. Vladimirov, Methods of the Theory of Functions of Several Complex Variables, M.I.T. Press, 1966. 
[100] Salomon Bochner and William Ted Martin, Several Complex Variables, Princeton Mathematical Series. Princeton University Press, 1948.

[101] B.V. Shabat, Introduction to complex analysis: Functions of several variables, Number pt. 2 in Translations of Mathematical Monographs. American Mathematical Society, 1992.

[102] J.B. Conway, Functions of one complex variable, Number v. 1 in Graduate texts in mathematics. Springer-Verlag, 1978.

[103] Friedrich E. P. Hirzebruch and Matthias Kreck, "On the concept of genus in topology and complex analysis," Notices of the American Mathematical Society, vol. 56, pp. 713-719.

[104] L.V. Ahlfors, Conformal Invariants: Topics in Geometric Function Theory, Ams Chelsea Publishing. AMS Chelsea Pub., 2010.

[105] R. Penrose, The road to reality: a complete guide to the laws of the universe, A Borzoi book. A.A. Knopf, 2005.

[106] Stefan Bergman, The kernel function and conformal mapping, American Mathematical Society, 1950.

[107] "Encyclopedia of Mathematics," http://eom.springer.de/.

[108] L. Hörmander, An introduction to complex analysis in several variables, University series in higher mathematics. Van Nostrand, 1966.

[109] V. V. Zharinov V. S. Vladimirov, "Analytic methods in mathematical physics," Proceedings of the Steklov Institute of Mathematics: Theoretical and Mathematical Physics, vol. 175, pp. 131ff., 1988.

[110] A. Dijksma B. Freydin D. Alpay, V. Bolotnikov, "Nonstationary analogues of the Herglotz representation theorem for unbounded operators," Archiv der Mathematik, vol. 269, pp. 465, 2002.

[111] Brodskiū, Triangular and Jordan representations of linear operators, vol. 32 of Transl. Math. Monographs, American Mathematical Society, 1971.

[112] Rolf Nevanlinna, Analytic Functions, Springer, 1970.

[113] F. Sommer H. Behnke, Theorie der analytischen Funktionen einer komplexer Veränderlichen, Transl. Math. Monographs. Springer, 1962.

[114] A. Korányi and L. Pukánszky, "Holomorphic Functions With Positive Real Part on Polycylinders," Transactions of the American Mathematical Society, vol. 108, no. 3, pp. pp. 449-456, 1963. 
[115] R.T. Cox, The algebra of probable inference, Johns Hopkins Press, 1961.

[116] Nailong Wu, The Maximum Entropy Method, Data and Knowledge in a Changing World. Springer, 1997.

[117] H. Jeffreys, "An Invariant Form for the Prior Probability in Estimation Problems," Royal Society of London Proceedings Series A, vol. 186, pp. 453-461, Sept. 1946.

[118] R. Bryan, "Maximum entropy analysis of oversampled data problems," European Biophysics Journal, vol. 18, pp. 165-174, 1990, 10.1007/BF02427376.

[119] S. F. Gull, "Developments in maximum-entropy data analysis," in Maximum Entropy and Bayesian Methods, J. Skilling, Ed., pp. 53-71. Kluwer Academic, Dordrecht, 1989.

[120] T. E. Bishop, S. D. Babacan, B. Amizic, A. K. Katsaggelos, T. Chan, and R. Molina, "Blind Image Deconvolution: problem formulation and existing approaches," in Blind image deconvolution: Theory and Applications, P. Campisi and K. Egiazarian, Eds., chapter 1. CRC Press, 2007.

[121] W. von der Linden, R. Preuss, and W. Hanke, "Consistent application of maximum entropy to quantum Monte Carlo data," Journal of Physics: Condensed Matter, vol. 8, no. 21, pp. 3881, 1996.

[122] Tatsuya Fujii and Kazuo Ueda, "Perturbative approach to the nonequilibrium Kondo effect in a quantum dot," Phys. Rev. B, vol. 68, pp. 155310, Oct 2003.

[123] A. Dirks, J.E. Han, M. Jarrell, and Th. Pruschke, "Imaginary-time quantum many-body theory out of equilibrium II: Analytic continuation of dynamic observables and transport properties," to be published.

[124] L. Hörmander, The analysis of linear partial differential operators, vol. 1 of Springer Study Edition, Springer-Verlag, 1990.

[125] Vasilii S. Vladimirov, V. V. Zharinov, and A. G. Sergeev, "Bogolyubov's 'edge of the wedge' theorem, its development and applications," Russian Mathematical Surveys, vol. 49, no. 5, pp. 51, 1994.

[126] L. Mühlbacher, D. F. Urban, and A. Komnik, "Anderson impurity model in nonequilibrium: Analytical results versus quantum Monte Carlo data," Phys. Rev. B, vol. 83, pp. 075107, Feb 2011.

[127] M. Jarrell, A. Macridin, K. Mikelsons, D. G. S. P. Doluweera, and J. E. Gubernatis, "The Dynamical Cluster Approximation with Quantum Monte Carlo Cluster Solvers," AIP Conference Proceedings, vol. 1014, no. 1, pp. 34-106, 2008 . 
[128] Matthew T Glossop and David E Logan, "Single-particle dynamics of the Anderson model: a local moment approach," Journal of Physics: Condensed Matter, vol. 14, no. 26, pp. 6737, 2002.

[129] M. Galassi, J. Davies, J. Theiler, B. Gough, G. Jungman, P. Alken, M. Booth, and F. Rossi, GNU Scientific Library Reference Manual , Network Theory Ltd., third edition, 2009. 



\section{Acknowledgements}

An erster Stelle sei meinem Doktorvater Thomas Pruschke gedankt, der stets ein offenes Ohr für auftretende Problemstellungen hatte. Auch während der teils sehr ausgeprägten Dürreperioden bei der Erstellung der vorliegenden Arbeit bewahrte er ein großes Maß an Zuversicht, das sich letztlich bezahlt machte.

Dank gilt zudem meinem Korreferenten Fakher Assaad, insbesondere für das Interesse und die Mühe, sich in die unkonventionellen mathematischen Details des Formalismus hineinzudenken.

Weiterer Dank gebührt Philipp Werner, ohne dessen sichere Ratschläge das Erlernen und Erörtern der Möglichkeiten der continuous-time-QMC-Methode im Rahmen der gegebenen Theorie nicht so schnell und ergiebig verlaufen wäre. Nicht unerwähnt bleiben darf seine außerordentliche Gastfreundschaft während meiner Aufenthalte in Zürich.

I am also indebted to Jong Han who from early on never ceased to provide many helpful insights into the chances and limitations of the approach. We had many discussions, sometimes cheerful, sometimes very serious. Altogether it was a great and exciting collaboration. Last but not least, I am grateful for his hospitality during my short stays at SUNY Buffalo and the MPI in Stuttgart.

Further thanks go to Mark Jarrell and his group who generously provided me with first-hand information about the MaxEnt method and their state-of-the-art largescale many-body simulation techniques. Again, I am grateful for their hospitality during my stays at LSU.

The time spent during the thesis was always accompanied by the friendly and productive atmosphere in the office with Navid, Sebastian, and Stephan. I will definitely miss the regular insights about tea ceremonies, cricket, music, movies, and many other concepts of cultural life.

I would like to thank Daniela Cadamuro, Dominik Wulff, Ebad Kamil, Riccardo Gezzi, and Sebastian Fuchs for their great help in proofreading the thesis.

$\mathrm{Zu}$ guter Letzt möchte ich mich für die moralische Unterstützung vieler Freunde bedanken, durch die diese Arbeit erst möglich geworden ist. Meinen Eltern danke ich für fortwährende Zuneigung und Förderung. 



\section{Curriculum vitae}

\section{Personal data}

Name: $\quad$ Andreas Dirks

Date of birth: December 02, 1982

Place of birth: Duderstadt, Germany

Nationality: German

\section{Education}

June 2002: $\quad$ Abitur, Freie Evangelische Bekenntnisschule Bremen

Oct 2003 - Mai 2008: $\quad$ studies in physics, University of Göttingen

June 2008 - June 2012: doctoral studies, University of Göttingen 
